\title{
Synthesis of Benzo-Fused Cyclic Ketones via Metal-Free Ring Expansion of Cyclopropanols Enabled by Proton-Coupled Electron Transfer
}

\author{
Tomohiro Kikuchi, Keiji Yamada, Takeshi Yasui,* and Yoshihiko Yamamoto* \\ Department of Basic Medicinal Sciences, Graduate School of Pharmaceutical Sciences, Nagoya \\ University, Furo-cho Chikusa, Nagoya 464-8603, Japan \\ t-yasui@ps.nagoya-u.ac.jp, yamamoto-yoshi@ps.nagoya-u.ac.jp
}

\section{Supporting Information}

Table of Contents

1. Optimization of Reaction Conditions $\quad$ S2

2. General Information $\quad$ S4

3. Synthesis and Characterization of 1-(2-alkenylaryl)cyclopropanols 1 S5

$\begin{array}{llr}\text { 4. Representative Procedure for the Ring Expansion of } \mathbf{1} & \text { S24 }\end{array}$

$\begin{array}{llr}\text { 5. Characterization of } \mathbf{4 a} & \text { S24 }\end{array}$

6. Characterization of 1-Tetralones $\mathbf{2}$ and 1-Benzosuberones $3 \quad$ S25

7. Radical Cyclization of the Alkyne Substrates 1o and 1p S32

8. Radical Cyclization of Cyclobutanol Substrate $\mathbf{5}$ and Cyclopentanol Substrate $\mathbf{6} \quad$ S35

9. Scale-Up Experiments $\quad$ S40

10. X-Ray Diffraction Analysis of $\mathbf{S 3 2}$ S41

11. DFT Calculations and Control Experiments $\quad$ S42

12. References $\quad$ S65

$\begin{array}{ll}\text { 13. NMR Spectra } & \text { S67 }\end{array}$ 


\section{Optimization of Reaction Conditions}

Table S1 Screening of bases<smiles>CCOC(=O)CCc1ccccc1C1(O)CC1</smiles>

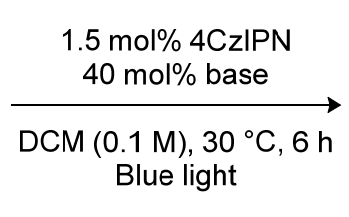<smiles>O=C(OCCC1CCC(=O)c2ccccc21)OCc1ccccc1</smiles><smiles>CCOC(=O)/C=C\c1ccccc1C1(O)CC1</smiles>

$1 a$

$$
\text { 2a }
$$

4a

\begin{tabular}{cccc} 
entry & base & $\mathbf{2 a}(\%)^{a, b}$ & $\mathbf{4 a ~}(\%)^{a, b}$ \\
\hline 1 & colidine & 15 & 16 \\
2 & $\mathrm{EtN}^{\prime} \mathrm{Pr}_{2}$ & $\mathrm{~N} . \mathrm{D}$. & 4 \\
3 & $\mathrm{PCy}_{3}$ & 17 & N.D. \\
4 & $\mathrm{P}^{n} \mathrm{Bu}_{3} \mathrm{Et}^{+}(\mathrm{EtO})_{2} \mathrm{POO}^{-}$ & 54 & 10 \\
5 & $\mathrm{P}^{n} \mathrm{Bu}_{4}{ }^{+} \mathrm{CF}_{3} \mathrm{COO}^{-}$ & 39 & N.D. \\
6 & $\mathrm{~N}^{n} \mathrm{Bu}_{4}{ }^{+} \mathrm{CF}_{3} \mathrm{COO}^{-}$ & 41 & 9 \\
7 & $\mathrm{P}^{n} \mathrm{Bu}_{4}{ }^{+}(n-\mathrm{BuO})_{2} \mathrm{POO}^{-}$ & 32 & 13 \\
8 & $\mathrm{~N}^{n} \mathrm{Bu}_{4}{ }^{+}(n-\mathrm{BuO})_{2} \mathrm{POO}^{-}$ & 40 & 6 \\
9 & $\mathrm{P}^{n} \mathrm{Bu}_{4}^{+}(\mathrm{PhO})_{2} \mathrm{POO}^{-}$ & 57 & 1 \\
10 & $\mathrm{~N}^{n} \mathrm{Bu}_{4}^{+}(\mathrm{PhO})_{2} \mathrm{POO}^{-}$ & 51 & N.D.
\end{tabular}

${ }^{a}$ NMR yield. ${ }^{b}$ N.D. $=$ Not detected.

Table S2 Influence of an amount of catalysts<smiles>CCOC(=O)CCc1ccccc1C1(O)CC1</smiles>

1a

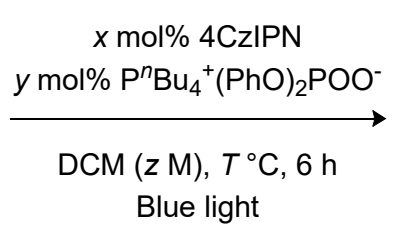

Blue light

\begin{tabular}{|c|c|c|c|c|c|c|c|}
\hline entry & $x(\%)$ & $y(\%)$ & $z(\mathrm{M})$ & $T\left({ }^{\circ} \mathrm{C}\right)$ & $2 \mathbf{a}(\%)^{a}$ & $\mathbf{4 a}(\%)^{a, b}$ & $\begin{array}{c}\text { recovered } \\
1 \mathbf{a}(\%)^{a, b}\end{array}$ \\
\hline 1 & 1.5 & 40 & 0.1 & 30 & 57 & 1 & N.D. \\
\hline 2 & 1.5 & 40 & 0.1 & 0 & $72(72)^{c}$ & N.D. & N.D. \\
\hline 3 & 1.5 & 40 & 0.05 & 30 & 63 & N.D. & N.D. \\
\hline 4 & 1.5 & 40 & 0.05 & 0 & 51 & 3 & 3 \\
\hline 5 & 1.5 & 40 & 0.2 & 0 & 52 & 1 & N.D. \\
\hline 6 & 1.5 & 100 & 0.1 & 0 & 63 & 2 & N.D. \\
\hline 7 & 1.5 & 200 & 0.1 & 0 & 53 & 6 & N.D. \\
\hline 8 & 3.0 & 40 & 0.1 & 0 & $76(85)^{c}$ & N.D. & N.D. \\
\hline 9 & 5.0 & 40 & 0.1 & 0 & 86 & N.D. & N.D. \\
\hline 10 & 10 & 40 & 0.1 & 0 & 77 & N.D. & N.D. \\
\hline 11 & 3.0 & 20 & 0.1 & 0 & 66 & N.D. & N.D. \\
\hline
\end{tabular}<smiles>CCOC(=O)CCC1CCC(=O)c2ccccc21</smiles>

$4 a$

${ }^{a}$ NMR yield. ${ }^{b}$ N.D. $=$ Not detected. ${ }^{c}$ Isolated yield. 
Table S3 Influence of solvents<smiles>CCOC(=O)C=Cc1ccccc1C1(O)CC1</smiles>

1a

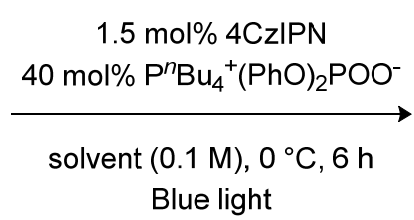

Blue light<smiles>CCOC(=O)CC1CCC(=O)c2ccccc21</smiles>

2a<smiles>CCOC(=O)/C=C\c1ccccc1C1(O)CC1</smiles>

$4 a$

\begin{tabular}{|c|c|c|c|}
\hline entry & solvent & $\mathbf{2} \mathbf{a}(\%)^{a}$ & $\mathbf{4 a}(\%)^{a, b}$ \\
\hline 1 & $\mathrm{DCM}$ & $86(85)$ & N.D. \\
\hline 2 & toluene & 34 & 22 \\
\hline 3 & THF & 8 & 19 \\
\hline 4 & $\mathrm{MeCN}$ & N.D. & 12 \\
\hline 5 & EtOAc & N.D. & 9 \\
\hline
\end{tabular}

${ }^{a}$ NMR yield. ${ }^{b}$ N.D. $=$ Not detected. ${ }^{c}$ Isolated yield.

Table S4 Control experiments<smiles>CCOC(=O)/C=C/c1ccccc1C1(O)CC1</smiles>

$1 \mathrm{a}$

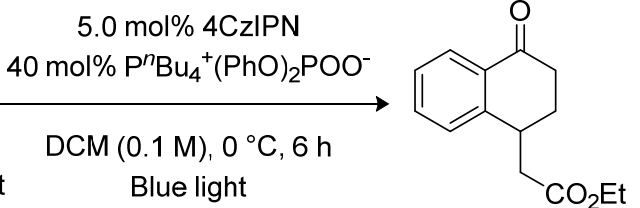

\begin{tabular}{ccc} 
entry & conditions & 2a (\%) $)^{a, b}$ \\
\hline 1 & no Blue light & N.D. \\
2 & no 4CzIPN & N.D. \\
3 & no base & N.D.
\end{tabular}

${ }^{a}$ NMR yield. ${ }^{b}$ N.D. $=$ Not detected .

Table S5 Influence of reaction temperature and HAT catalysts in the reaction using 1r<smiles>OC1(c2ccccc2C(=[Te])c2ccccc2)CC1</smiles>

$1 \mathrm{r}$

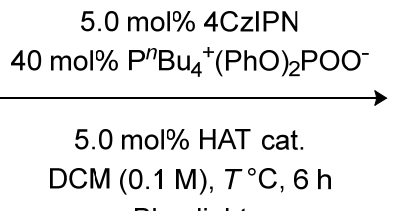

Blue light<smiles>O=C1CCCC(c2ccccc2)c2ccccc21</smiles>

$3 r$

\begin{tabular}{cccc} 
entry & $T\left({ }^{\circ} \mathrm{C}\right)$ & HAT cat. & $\mathbf{3 r}(\%)^{a}$ \\
\hline 1 & 0 & - & $18^{b}$ \\
2 & 30 & - & 51 \\
3 & 30 & TRIP-SH $^{c}$ & 50 \\
4 & 30 & NHPI $^{d}$ & 38
\end{tabular}

${ }^{a} \mathrm{NMR}$ yield. ${ }^{b}$ The reaction was performed for $14 \mathrm{~h}$. $1 \mathrm{p}$ was recovered in $56 \%$ yield. ${ }^{c} 2,4,6$-triisopropyl thiophenol. ${ }^{d} N$-hydroxyphthalimide.

$5.0 \mathrm{~mol} \%$ 4CzIPN<smiles>CCOC(=O)C=Cc1ccccc1C1(O)CCC1</smiles>

$5(n=1)$

$6(n=2)$
$40 \mathrm{~mol}^{n}{ }^{n} \mathrm{Bu}_{3} \mathrm{EtP}^{+}(\mathrm{EtO})_{2} \mathrm{POO}^{-}$(for 5)

$40 \mathrm{~mol}^{\circ}{ }^{n} \mathrm{Bu}_{4} \mathrm{P}^{+}(\mathrm{PhO})_{2} \mathrm{POO}^{-}$(for 6)

DCM, $\boldsymbol{T}^{\circ} \mathrm{C}, 17 \mathrm{~h}$

Blue light

conv. $>99 \%$

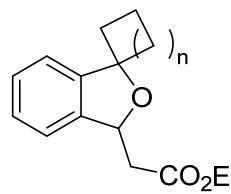

$7(n=1)$

$8(n=2)$

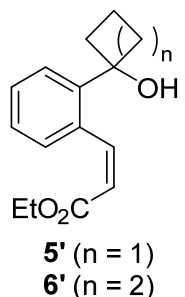

\begin{tabular}{ccc}
\hline & $\mathbf{7}$ or $\mathbf{8}$ & $\mathbf{5}^{\prime}$ or $\mathbf{6}$ \\
\hline$n=1, T=30^{\circ} \mathrm{C}$ & $39 \%$ & detected \\
$n=1, T=0^{\circ} \mathrm{C}$ & $2 \%$ & major \\
$n=2, T=30^{\circ} \mathrm{C}$ & $68 \%$ & detected \\
$n=2, T=0^{\circ} \mathrm{C}$ & $38 \%$ & major
\end{tabular}

Scheme S1 Reactions of 5 and 6 


\section{General information}

General considerations: All air- and moisture-sensitive reactions were performed under an argon (Ar) atmosphere. Analytical thin layer chromatography was performed using $0.25 \mathrm{~mm}$ silica gel plate (Merck TLC Silica gel $60 \mathrm{~F}_{254}$ ). Column chromatography was performed on silica gel (Cica silica gel $60 \mathrm{~N}$ ) with solvents specified below. Melting points were recorded on SRS OptiMelt MPA100. NMR spectra were recorded on JEOL ESC-400 spectrometer $\left({ }^{1} \mathrm{H} / 400 \mathrm{MHz}\right.$ and $\left.{ }^{13} \mathrm{C} / 101 \mathrm{MHz}\right)$ for samples in $\mathrm{CDCl}_{3}$ solutions at $25{ }^{\circ} \mathrm{C} .{ }^{1} \mathrm{H}$ NMR chemical shifts are reported in terms of chemical shift $(\delta, \mathrm{ppm})$ relative to the signal at $\delta 0.00 \mathrm{ppm}$ for internal tetramethylsilane. ${ }^{13} \mathrm{C}$ NMR spectra were fully decoupled and are reported in terms of chemical shift $(\delta, \mathrm{ppm})$ relative to the triplet at $\delta 77.0 \mathrm{ppm}$ for $\mathrm{CDCl}_{3} .{ }^{19} \mathrm{~F}$ NMR spectra are reported in terms of chemical shift $(\delta, \mathrm{ppm})$ relative to the singlet at $\delta-63.7 \mathrm{ppm}$ for $a, a, a$-trifluorotoluene as an external standard. Splitting patterns are designated as follows: s, singlet; d, doublet; t, triplet; q, quartet; quint, quintet; sext, sextet; sept, septet; m, multiplet. Coupling constants are reported in Hz. Infrared spectra were recorded on JASCO FT/IR230 spectrometer. High-resolution mass spectra were recorded on JEOL JMS-T100LP (TOF) mass spectrometer. As a light source, PER-AMP available from Techno Sigma (448 nm: 680 mW, 385 nm: 490 $\mathrm{mW}$ ) was used. The LED light, which is covered by borosilicate glass tube, was directly put into the solvent and the reaction mixture was irradiated from inside the reaction solution.

Reagents and Solvents: Photocatalysts $\left.\left[\operatorname{Ir}\left(\mathrm{dF}_{(\mathrm{CF}}\right) \text { ppy }\right)_{2}(\mathrm{dtbbpy})\right]\left(\mathrm{PF}_{6}\right),{ }^{1} 4 \mathrm{DPAIPN},{ }^{2} 4 \mathrm{CzPN}^{2}$, and $4 \mathrm{CzIPN}^{2}$ were prepared according to the report. All other phosphates excepted $\mathrm{P}^{n} \mathrm{Bu}_{3} \mathrm{Et}^{+}(\mathrm{EtO})_{2} \mathrm{POO}^{-}$were prepared using a method reported by Knowles and co-worker. ${ }^{3}$ Dry DCM was distilled from calcium hydride under Ar atmosphere and stored over $4 \AA$ molecular sieves. Other solvents and reagents were purchased from chemical suppliers (Aldrich, Kanto Chemical, TCI, and Wako) and used as received. 
3. Synthesis and Characterization of 1-(2-alkenylaryl)cyclopropanols 1

Synthesis of $1 \mathrm{a}-\mathbf{i}$

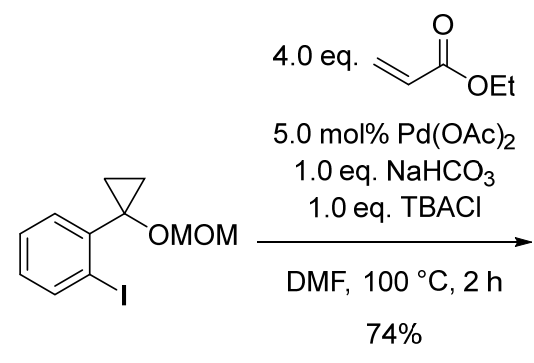

S1<smiles>CCOC(=O)/C=C/c1ccccc1C1(OC)CC1</smiles>

S2a

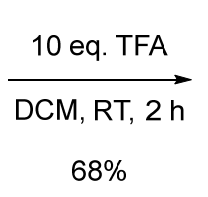

$68 \%$

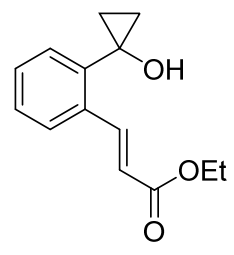

$1 \mathrm{a}$

S1 was prepared according to the previous report. ${ }^{4}$

\section{Synthesis and characterization of S2a-i}<smiles>CCOC(=O)/C=C/c1ccccc1C1(OC)CC1</smiles>

S2a

To a solution of $\mathbf{S 1}(304 \mathrm{~g}, 1.00 \mathrm{mmol})$ in DMF $(2.00 \mathrm{~mL})$ was added $\mathrm{Pd}(\mathrm{OAc})_{2}(11.3 \mathrm{mg}$, $0.0500 \mathrm{mmol}), \mathrm{NaHCO}_{3}(84.1 \mathrm{mg}, 1.00 \mathrm{mmol})$ and TBACl $(278 \mathrm{mg}, 1.00 \mathrm{mmol})$. After degassed at $-78{ }^{\circ} \mathrm{C}$, the mixture was added Ethyl acrylate $(440 \mu \mathrm{L}, 4.05 \mathrm{mmol})$. The reaction mixture was stirred at $100{ }^{\circ} \mathrm{C}$ for $2 \mathrm{~h}$ using an oil bath. After that, the reaction mixture was filtered through a pad of silica gel with a mixed solution of hexane $(40 \mathrm{~mL})$ and EtOAc $(10$ $\mathrm{mL}$ ). The filtrate concentrated in vacuo. The obtained crude product was purified by silica gel column chromatography (Hexane/EtOAc $=20: 1)$ to give $\mathbf{S 2 a}(205 \mathrm{mg}, 74 \%)$. Analytical data for S2a: yellow oil; ${ }^{1} \mathrm{H}$ NMR (400 MHz, $\left.\mathrm{CDCl}_{3}\right) \delta 8.52(\mathrm{~d}, J=16.0 \mathrm{~Hz}, 1 \mathrm{H}), 7.65-7.63(\mathrm{~m}, 1 \mathrm{H}), 7.41-7.38(\mathrm{~m}, 1 \mathrm{H}), 7.32-7.29$ (m, 2H), $6.43(\mathrm{~d}, J=16.0 \mathrm{~Hz}, 1 \mathrm{H}), 4.55(\mathrm{~s}, 2 \mathrm{H}), 4.27$ (q, $J=7.2 \mathrm{~Hz}, 2 \mathrm{H}), 3.12(\mathrm{~s}, 3 \mathrm{H}), 1.34$ (t, $J=7.2 \mathrm{~Hz}, 3 \mathrm{H})$, $1.30(\mathrm{dd}, J=7.2,5.2 \mathrm{~Hz}, 2 \mathrm{H}), 0.93(\mathrm{dd}, J=7.2,5.2 \mathrm{~Hz}, 2 \mathrm{H}) ;{ }^{13} \mathrm{C}-\mathrm{NMR}\left(101 \mathrm{MHz}, \mathrm{CHCl}_{3}\right) \delta 167.0,142.8$, 139.4, 135.4, 130.3, 129.5, 128.4, 126.6, 119.3, 94.9, 60.5, 60.4, 55.6, 14.4, 12.5; IR (neat) 1714, $1177 \mathrm{~cm}^{-1}$; HRMS (DART) $m / z:\left[\mathrm{M}+\mathrm{NH}_{4}\right]^{+}$Calcd for $\mathrm{C}_{16} \mathrm{H}_{24} \mathrm{NO}_{4} 294.1705$; Found 294.1693.<smiles>COC(=O)/C=C/c1ccccc1C1(OC)CC1</smiles>

S2b

This compound was purified by silica gel column chromatography (Hexane/EtOAc $=20: 1)$. Analytical data for S2b: pale-yellow oil; ${ }^{1} \mathrm{H}-\mathrm{NMR}\left(400 \mathrm{MHz}, \mathrm{CDCl}_{3}\right) \delta 8.51(\mathrm{~d}, J=16.0$ $\mathrm{Hz}, 1 \mathrm{H}), 7.64(\mathrm{dd}, J=5.6,3.6 \mathrm{~Hz}, 1 \mathrm{H}), 7.40(\mathrm{dd}, J=5.6,3.6 \mathrm{~Hz}, 1 \mathrm{H}), 7.32-7.30(\mathrm{~m}, 2 \mathrm{H})$, $6.44(\mathrm{~d}, J=16.0 \mathrm{~Hz}, 1 \mathrm{H}), 4.55(\mathrm{~s}, 2 \mathrm{H}), 3.81(\mathrm{~s}, 3 \mathrm{H}), 3.12(\mathrm{~s}, 3 \mathrm{H}), 1.30(\mathrm{dd}, J=7.2,5.2 \mathrm{~Hz}$, $2 \mathrm{H}), 0.93(\mathrm{dd}, J=7.2,5.2 \mathrm{~Hz}, 2 \mathrm{H}) ;{ }^{13} \mathrm{C}-\mathrm{NMR}\left(101 \mathrm{MHz}, \mathrm{CHCl}_{3}\right) \delta 167.4,143.0,139.4,135.4$, 130.2, 129.6, 128.4 126.6, 118.9, 94.9, 60.5, 55.6, 51.7, 12.5; IR (neat) 1719, $1173 \mathrm{~cm}^{-1}$; HRMS (DART) $m / z$ : $[\mathrm{M}+\mathrm{H}]^{+}$Calcd for $\mathrm{C}_{15} \mathrm{H}_{19} \mathrm{O}_{4} 263.1283$; Found 263.1265. 


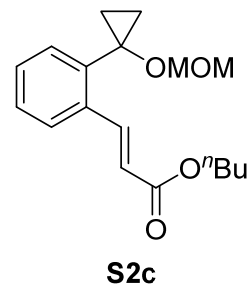

This compound was purified by silica gel column chromatography (Hexane/EtOAc $=20: 1)$ and obtained as a mixture with a trace amount of $(Z)$-isomer. Analytical data for S2c: paleyellow oil; ${ }^{1} \mathrm{H}-\mathrm{NMR}\left(400 \mathrm{MHz}, \mathrm{CDCl}_{3}\right) \delta 8.53(\mathrm{~d}, J=16.0 \mathrm{~Hz}, 1 \mathrm{H}), 7.66-7.63(\mathrm{~m}, 1 \mathrm{H})$, $7.41-7.38(\mathrm{~m}, 1 \mathrm{H}), 7.32-7.29(\mathrm{~m}, 2 \mathrm{H}), 6.43(\mathrm{~d}, J=16.0 \mathrm{~Hz}, 1 \mathrm{H}), 4.55(\mathrm{~s}, 2 \mathrm{H}), 4.23(\mathrm{t}, J=$ $6.8 \mathrm{~Hz}, 2 \mathrm{H}), 3.12(\mathrm{~s}, 3 \mathrm{H}), 1.74-1.67(\mathrm{~m}, 2 \mathrm{H}), 1.48-1.44(\mathrm{~m}, 2 \mathrm{H}), 1.30$ (dd, J= 7.2, $5.2 \mathrm{~Hz}$, 2H), 0.97 (t, $J=7.6 \mathrm{~Hz}, 3 \mathrm{H}), 0.93$ (dd, $J=7.2,5.2 \mathrm{~Hz}, 2 \mathrm{H}) ;{ }^{13} \mathrm{C}-\mathrm{NMR}\left(101 \mathrm{MHz}, \mathrm{CHCl}_{3}\right) \delta 167.1,142.7$, 139.4, 135.4, 130.2, 129.5, 128.4, 126.5, 119.2, 94.9, 64.3, 60.5, 55.6, 30.8, 19.3, 13.8, 12.5; IR (neat) 1715, $1173 \mathrm{~cm}^{-1}$; HRMS (DART) $\mathrm{m} / z$ : $\left[\mathrm{M}+\mathrm{NH}_{4}\right]^{+}$Calcd for $\mathrm{C}_{18} \mathrm{H}_{28} \mathrm{NO}_{4} 322.2018$; Found 322.2034.

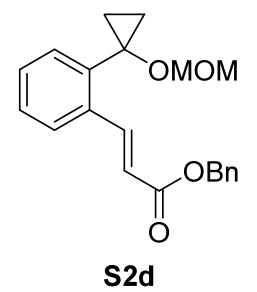

Benzyl acrylate was prepared according to the previous report. ${ }^{5}$ This compound was purified by silica gel column chromatography $($ Hexane/EtOAc $=20: 1)$. Analytical data for S2d: pale-yellow oil; ${ }^{1} \mathrm{H}-\mathrm{NMR}\left(400 \mathrm{MHz}, \mathrm{CDCl}_{3}\right) \delta 8.60(\mathrm{~d}, J=16.0 \mathrm{~Hz}, 1 \mathrm{H}), 7.63-7.61(\mathrm{~m}, 1 \mathrm{H})$, 7.42-7.27 (m, 8H), 6.48 (d, $J=16.0 \mathrm{~Hz}, 1 \mathrm{H}), 5.26(\mathrm{~s}, 2 \mathrm{H}), 4.53(\mathrm{~s}, 2 \mathrm{H}), 3.09$ (s, 3H), 1.29 $(\mathrm{dd}, J=7.2,5.2 \mathrm{~Hz}, 2 \mathrm{H}), 0.91(\mathrm{dd}, J=7.2,5.2 \mathrm{~Hz}, 2 \mathrm{H}) ;{ }^{13} \mathrm{C}-\mathrm{NMR}\left(101 \mathrm{MHz}, \mathrm{CHCl}_{3}\right) \delta 166.8$, 143.5, 139.6, 136.4, 135.3, 130.3, 129.7, 128.7, 128.5, 128.3, 128.2, 126.7, 118.9, 95.0, 66.3, 60.6, 55.7, 12.6; IR (neat) 1714, $1173 \mathrm{~cm}^{-1}$; HRMS (DART) $\mathrm{m} / z$ : $\left[\mathrm{M}+\mathrm{NH}_{4}\right]^{+}$Calcd for $\mathrm{C}_{21} \mathrm{H}_{26} \mathrm{NO}_{4}$ 356.1862; Found 356.1889.

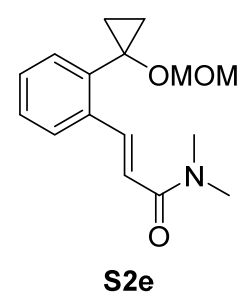

This compound was purified by silica gel column chromatography (Hexane/EtOAc $=10: 1)$. Analytical data for S2e: brown oil; ${ }^{1} \mathrm{H}-\mathrm{NMR}\left(400 \mathrm{MHz}, \mathrm{CDCl}_{3}\right) \delta 8.35(\mathrm{~d}, J=15.6 \mathrm{~Hz}, 1 \mathrm{H})$, 7.64-7.61 (m, 1H), 7.42-7.39 (m, 1H), 7.32-7.28 (m, 2H), 6.89 (d, J=15.6 Hz, 1H), $4.56(\mathrm{~s}$, $2 \mathrm{H}), 3.19(\mathrm{~s}, 3 \mathrm{H}), 3.13(\mathrm{~s}, 3 \mathrm{H}), 3.08(\mathrm{~s}, 3 \mathrm{H}), 1.31$ (dd, $J=7.2,5.6 \mathrm{~Hz}, 2 \mathrm{H}), 0.94$ (dd, $J=7.2$, $5.6 \mathrm{~Hz}, 2 \mathrm{H}) ;{ }^{13} \mathrm{C}-\mathrm{NMR}\left(101 \mathrm{MHz}, \mathrm{CHCl}_{3}\right) \delta 167.1,140.2,138.9,136.4,130.4,128.8,128.3$, 126.7, 119.0, 94.8, 60.7, 55.6, 37.6, 35.9, 12.6; IR (neat) 1650, $1152 \mathrm{~cm}^{-1}$; HRMS (DART) $\mathrm{m} / z$ : [M+H] Calcd for $\mathrm{C}_{16} \mathrm{H}_{22} \mathrm{NO}_{3}$ 276.1600; Found 276.1609.

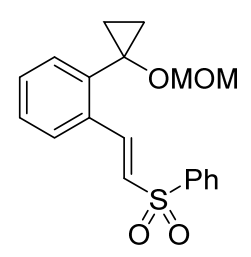

S2f

(Vinylsulfonyl)benzene was prepared according to the previous report. ${ }^{6} 22 \mathrm{~mol} \%(o-\mathrm{Tol})_{3} \mathrm{P}$ was used as a ligand in addition to representative conditions. This compound was purified by silica gel column chromatography $(\mathrm{Hexane} / \mathrm{EtOAc}=10: 1)$. Analytical data for S2f: paleyellow oil; ${ }^{1} \mathrm{H}-\mathrm{NMR}\left(400 \mathrm{MHz}, \mathrm{CDCl}_{3}\right) \delta 8.45(\mathrm{~d}, J=15.6 \mathrm{~Hz}, 1 \mathrm{H}), 7.99(\mathrm{dt}, J=6.8,1.6 \mathrm{~Hz}$, 2H), 7.64-7.59 (m, 1H), $7.54(\mathrm{dt}, J=9.2,1.6 \mathrm{~Hz}, 2 \mathrm{H}), 7.51(\mathrm{dd}, J=9.2,1.6 \mathrm{~Hz}, 1 \mathrm{H}), 7.40$ $(\mathrm{dd}, J=7.2,1.6 \mathrm{~Hz}, 1 \mathrm{H}), 7.36-7.28(\mathrm{~m}, 2 \mathrm{H}), 6.87(\mathrm{~d}, J=15.6 \mathrm{~Hz}, 1 \mathrm{H}), 4.52(\mathrm{~s}, 2 \mathrm{H}), 3.07$ (s, 3H), 1.29 (dd, $J$ $=7.2,5.2 \mathrm{~Hz}, 2 \mathrm{H}), 0.92(\mathrm{dd}, J=7.3,5.2 \mathrm{~Hz}, 2 \mathrm{H}) ;{ }^{13} \mathrm{C}-\mathrm{NMR}\left(101 \mathrm{MHz}, \mathrm{CHCl}_{3}\right) \delta 141.5,141.0,140.0,133.6$, 133.3, 130.4, 130.2, 129.3, 128.7, 128.6, 127.9, 127.4, 95.0, 60.5, 55.7, 12.6; IR (neat) 1307, $1147 \mathrm{~cm}^{-1}$; HRMS (DART) $\mathrm{m} / \mathrm{z}:\left[\mathrm{M}+\mathrm{NH}_{4}\right]^{+}$Calcd for $\mathrm{C}_{19} \mathrm{H}_{21} \mathrm{O}_{4} \mathrm{~S}$ 345.1161; Found 345.1165. 


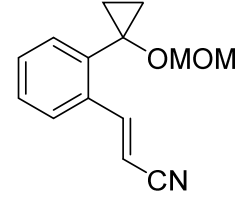

S2g

This compound was purified by silica gel column chromatography $($ Hexane/EtOAc $=20: 1)$ and obtained as a mixture of $E / Z$ isomer $(E / Z=2.5: 1)$. Analytical data for $\mathbf{S 2 g}$ : yellow oil; ${ }^{1} \mathrm{H}-\mathrm{NMR}\left(400 \mathrm{MHz}, \mathrm{CDCl}_{3}\right)$ major $(\boldsymbol{E}) \delta 8.26(\mathrm{~d}, J=16.4 \mathrm{~Hz}, 1 \mathrm{H}), 7.56-7.54(\mathrm{~m}, 1 \mathrm{H}), 7.44$ $7.32(\mathrm{~m}, 3 \mathrm{H}), 5.90(\mathrm{~d}, J=16.4 \mathrm{~Hz}, 1 \mathrm{H}), 4.55(\mathrm{~s}, 2 \mathrm{H}), 3.13(\mathrm{~s}, 3 \mathrm{H}), 1.29(\mathrm{dd}, J=7.2,5.2 \mathrm{~Hz}$, $2 \mathrm{H}), 0.93(\mathrm{dd}, J=7.2,5.2 \mathrm{~Hz}, 2 \mathrm{H})$; $\operatorname{minor}(Z) \delta 8.11-8.08(\mathrm{~m}, 1 \mathrm{H}), 8.01(\mathrm{~d}, J=12.0 \mathrm{~Hz}, 1 \mathrm{H})$, 7.44-7.32 (m, 3H), $5.56(\mathrm{~d}, J=12.0 \mathrm{~Hz}, 1 \mathrm{H}), 4.55(\mathrm{~s}, 2 \mathrm{H}), 3.14(\mathrm{~s}, 3 \mathrm{H}), 1.25(\mathrm{dd}, J=7.2,5.2 \mathrm{~Hz}, 2 \mathrm{H}), 0.96$ $(\mathrm{dd}, J=7.2,5.2 \mathrm{~Hz}, 2 \mathrm{H}),{ }^{13} \mathrm{C}-\mathrm{NMR}\left(101 \mathrm{MHz}, \mathrm{CHCl}_{3}\right)$ major $(\boldsymbol{E}) \delta 149.1,139.3,134.4,130.6,130.3,128.6$, $125.9,97.1,95.1,60.46,55.8,12.54$ minor $(Z) \delta 148.0,139.2,134.4,130.3,129.8,128.6,128.2,96.2,95.2$, 60.55, 55.9, 12.51; IR (neat) 2218, $1154 \mathrm{~cm}^{-1}$; HRMS (DART) $\mathrm{m} / z$ : $\left[\mathrm{M}+\mathrm{NH}_{4}\right]^{+}$Calcd for $\mathrm{C}_{14} \mathrm{H}_{19} \mathrm{~N}_{2} \mathrm{O}_{2}$ 247.1447; Found 247.1425.

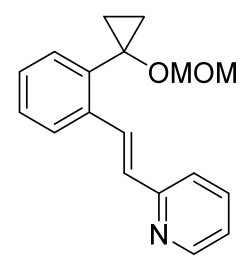

S2h

2-Vinylpyridine was prepared according to the previous report. ${ }^{7} 22 \mathrm{~mol} \%(o-\mathrm{Tol})_{3} \mathrm{P}$ was used as a ligand in addition to representative conditions. This compound was purified by silica gel column chromatography $($ Hexane/EtOAc $=20: 1)$. Analytical data for $\mathbf{S 2 h}$ : yellow oil; ${ }^{1} \mathrm{H}-$ $\operatorname{NMR}\left(400 \mathrm{MHz}, \mathrm{CDCl}_{3}\right) \delta 8.60(\mathrm{dd}, J=4.8,1.2 \mathrm{~Hz}, 1 \mathrm{H}), 8.32(\mathrm{~d}, J=16.4 \mathrm{~Hz}, 1 \mathrm{H}), 7.77(\mathrm{dd}$, $J=8.0,1.2 \mathrm{~Hz}, 1 \mathrm{H}), 7.65(\mathrm{td}, J=8.0,1.2 \mathrm{~Hz}, 1 \mathrm{H}), 7.55(\mathrm{~d}, J=8.0 \mathrm{~Hz}, 1 \mathrm{H}), 7.39$ (dd, $J=8.0$, $1.2 \mathrm{~Hz}, 1 \mathrm{H}), 7.33(\mathrm{td}, J=7.6,1.2 \mathrm{~Hz}, 1 \mathrm{H}), 7.25(\mathrm{td}, J=7.6,1.2 \mathrm{~Hz}, 1 \mathrm{H}), 7.20(\mathrm{~d}, J=16.4$ Hz, 1H), 7.12 (ddd, $J=7.6,4.8,1.2 \mathrm{~Hz}, 1 \mathrm{H}), 4.59$ (s, 2H), 3.16 (s, 3H), 1.31 (dd, $J=7.2,5.2 \mathrm{~Hz}, 2 \mathrm{H}), 0.98$ $(\mathrm{dd}, J=7.2,5.2 \mathrm{~Hz}, 2 \mathrm{H}) ;{ }^{13} \mathrm{C}-\mathrm{NMR}\left(101 \mathrm{MHz}, \mathrm{CHCl}_{3}\right) \delta 156.4,149.8,137.8,137.6,136.5,130.9,130.3,129.6$, $128.5,127.8,125.9,122.0,121.3,94.9,60.8,55.8,12.7$; IR (neat) $1153 \mathrm{~cm}^{-1}$; HRMS (DART) $m / z:[\mathrm{M}+\mathrm{H}]^{+}$ Calcd for $\mathrm{C}_{18} \mathrm{H}_{20} \mathrm{NO}_{2} 282.1494$; Found 282.1500.

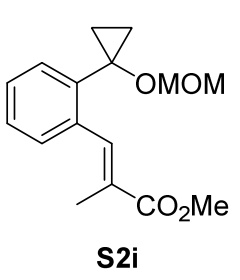

This compound was purified by silica gel column chromatography (Hexane/EtOAc $=20: 1)$. Analytical data for S2i: yellow oil; ${ }^{1} \mathrm{H}-\mathrm{NMR}\left(400 \mathrm{MHz}, \mathrm{CDCl}_{3}\right) \delta 8.22(\mathrm{~s}, 1 \mathrm{H}), 7.41$ (dd, $J$ $=6.8,0.8 \mathrm{~Hz}, 1 \mathrm{H}), 7.32-7.27(\mathrm{~m}, 3 \mathrm{H}), 4.55(\mathrm{~s}, 2 \mathrm{H}), 3.84(\mathrm{~s}, 3 \mathrm{H}), 3.14(\mathrm{~s}, 3 \mathrm{H}), 2.02(\mathrm{~s}, 3 \mathrm{H})$, $1.21(\mathrm{dd}, J=7.2,5.6 \mathrm{~Hz}, 2 \mathrm{H}), 0.90(\mathrm{dd}, J=7.2,5.6 \mathrm{~Hz}, 2 \mathrm{H}) ;{ }^{13} \mathrm{C}-\mathrm{NMR}\left(101 \mathrm{MHz}, \mathrm{CHCl}_{3}\right)$ $\delta 169.3,138.8,138.6,137.1,129.9,129.7,128.2,127.9,127.8,94.9,60.9,55.7,52.2,14.2$, 12.5; IR (neat) 1713, $1154 \mathrm{~cm}^{-1}$; HRMS (DART) $\mathrm{m} / z$ : [M $\left.+\mathrm{NH}_{4}\right]^{+}$Calcd for $\mathrm{C}_{16} \mathrm{H}_{24} \mathrm{NO}_{4}$ 294.1705; Found 294.1685 .

\section{Synthesis and characterization of $1 \mathrm{a}-\mathbf{i}$}

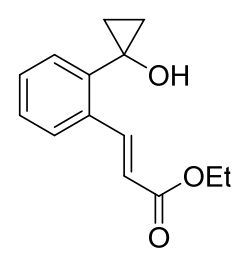

1a

To a $50 \mathrm{~mL}$ flask was added S2a (205 mg, $0.740 \mathrm{mmol})$ followed by degassed DCM (10 mL). TFA (560 $\mu \mathrm{L}, 7.32 \mathrm{mmol}$ ) was slowly added to the solution. After being stirred at $\mathrm{rt}$ for $2 \mathrm{~h}$ under Ar atmosphere, the reaction mixture was diluted with water and extracted with EtOAc. The organic layer was washed with sat. aq. $\mathrm{NaHCO}_{3}$ and brine, dried over $\mathrm{MgSO}_{4}$, and concentrated in vacuo. The residue was purified by silica gel column chromatography $($ Hexane/EtOAc $=10: 1)$ to furnish $1 \mathrm{a}(117 \mathrm{mg}, 68 \%)$. Analytical data for 1a: yellow oil; ${ }^{1} \mathrm{H}-$ 
NMR (400 MHz, $\left.\mathrm{CDCl}_{3}\right) \delta 8.44(\mathrm{~d}, J=16.0 \mathrm{~Hz}, 1 \mathrm{H}), 7.58-7.56(\mathrm{~m}, 1 \mathrm{H}), 7.40-7.37(\mathrm{~m}, 1 \mathrm{H}), 7.30-7.24(\mathrm{~m}$, 2H), 6.35 (d, $J=16.0 \mathrm{~Hz}, 1 \mathrm{H}), 4.19$ (q, $J=7.2 \mathrm{~Hz}, 2 \mathrm{H}), 3.91$ (s, 1H), 1.29 (t, $J=7.2 \mathrm{~Hz}, 3 \mathrm{H}), 1.19$ (dd, $J=$ 7.2, $5.2 \mathrm{~Hz}, 2 \mathrm{H}), 0.87(\mathrm{dd}, J=7.2,5.2 \mathrm{~Hz}, 2 \mathrm{H}) ;{ }^{13} \mathrm{C}-\mathrm{NMR}\left(101 \mathrm{MHz}, \mathrm{CDCl}_{3}\right) \delta 167.5,142.8,141.7,134.7$, 130.0, 129.6, 128.3, 126.6, 119.1, 60.7, 56.0, 14.3, 14.0; IR (neat) 3421, $1697 \mathrm{~cm}^{-1}$; HRMS (DART) $\mathrm{m} / z$ : [M $\left.+\mathrm{NH}_{4}\right]^{+}$Calcd for $\mathrm{C}_{14} \mathrm{H}_{20} \mathrm{NO}_{3} 250.1443$; Found 250.1420 .

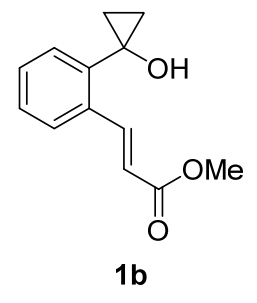

This compound was purified by silica gel column chromatography $($ Hexane/EtOAc $=10: 1)$. Analytical data for 1b: pale-yellow gum; ${ }^{1} \mathrm{H}-\mathrm{NMR}\left(400 \mathrm{MHz}, \mathrm{CDCl}_{3}\right) \delta 8.45$ (d, $J=16.0$ $\mathrm{Hz}, 1 \mathrm{H}), 7.62-7.59$ (m, 1H), 7.43-7.41 (m, 1H), 7.34-7.28 (m, 2H), 6.39 (d, $J=16.0 \mathrm{~Hz}$, $1 \mathrm{H}), 3.77(\mathrm{~s}, 3 \mathrm{H}), 3.33(\mathrm{~s}, 1 \mathrm{H}), 1.23(\mathrm{dd}, J=7.2,5.2 \mathrm{~Hz}, 2 \mathrm{H}), 0.92(\mathrm{dd}, J=7.2,5.2 \mathrm{~Hz}, 2 \mathrm{H})$;

${ }^{13} \mathrm{C}-\mathrm{NMR}\left(101 \mathrm{MHz}, \mathrm{CDCl}_{3}\right) \delta 167.8,142.9,141.5,134.8,130.1,129.4,128.4,126.7,118.9$, 56.2, 51.9, 14.1; IR (neat) 3421, $1698 \mathrm{~cm}^{-1}$; HRMS (DART) $\mathrm{m} / z$ : $\left[\mathrm{M}+\mathrm{NH}_{4}\right]^{+}$Calcd for $\mathrm{C}_{13} \mathrm{H}_{18} \mathrm{NO}_{3} 236.1287$; Found 236.1269.

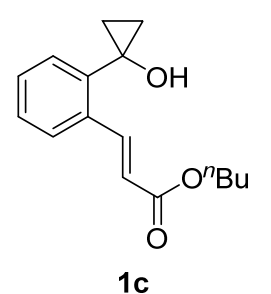

This compound was purified by silica gel column chromatography (Hexane/EtOAc $=10: 1)$. Analytical data for 1c: pale- yellow oil; ${ }^{1} \mathrm{H}-\mathrm{NMR}\left(400 \mathrm{MHz}, \mathrm{CDCl}_{3}\right) \delta 8.46(\mathrm{~d}, J=16.0$ $\mathrm{Hz}, 1 \mathrm{H}), 7.63-7.61(\mathrm{~m}, 1 \mathrm{H}), 7.44-7.41(\mathrm{~m}, 1 \mathrm{H}), 7.34-7.29(\mathrm{~m}, 2 \mathrm{H}), 6.41(\mathrm{~d}, J=16.0 \mathrm{~Hz}$, $1 \mathrm{H}), 4.19(\mathrm{t}, J=6.8 \mathrm{~Hz}, 2 \mathrm{H}), 3.06(\mathrm{~s}, 1 \mathrm{H}), 1.69(\mathrm{tt}, J=7.2,6.8 \mathrm{~Hz}, 2 \mathrm{H}), 1.44(\mathrm{qt}, J=7.2,7.2$ $\mathrm{Hz}, 2 \mathrm{H}), 1.23$ (dd, $J=7.2,5.2 \mathrm{~Hz}, 2 \mathrm{H}), 0.97$ (t, $J=7.2 \mathrm{~Hz}, 3 \mathrm{H}), 0.93(\mathrm{dd}, J=7.2,5.2 \mathrm{~Hz}$, 2H); ${ }^{13} \mathrm{C}-\mathrm{NMR}\left(101 \mathrm{MHz}, \mathrm{CDCl}_{3}\right) \delta 167.4,142.5,141.4,134.9,130.0,129.4,128.5,126.7,119.5,64.6,56.3$, 30.8, 19.3, 14.1, 13.9; IR (neat) 3422, $1697 \mathrm{~cm}^{-1}$; HRMS (DART) $\mathrm{m} / z$ : [M+ H] ${ }^{+}$Calcd for $\mathrm{C}_{16} \mathrm{H}_{21} \mathrm{O}_{3} 261.1491$; Found 261.1481.

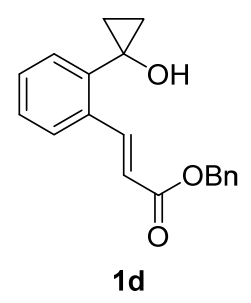

This compound was purified by silica gel column chromatography (Hexane/EtOAc $=10: 1)$. Analytical data for 1d: pale- yellow oil; ${ }^{1} \mathrm{H}-\mathrm{NMR}\left(400 \mathrm{MHz}, \mathrm{CDCl}_{3}\right) \delta 8.52(\mathrm{~d}, J=16.0 \mathrm{~Hz}$, $1 \mathrm{H}), 7.58(\mathrm{dd}, J=6.8,1.6 \mathrm{~Hz}, 1 \mathrm{H}), 7.40-7.26(\mathrm{~m}, 8 \mathrm{H}), 6.43(\mathrm{~d}, J=16.0 \mathrm{~Hz}, 1 \mathrm{H}), 5.20$ (s, $2 \mathrm{H}), 3.42(\mathrm{~s}, 1 \mathrm{H}), 1.20(\mathrm{dd}, J=7.2,5.2 \mathrm{~Hz}, 2 \mathrm{H}), 0.89(\mathrm{dd}, J=7.2,5.2 \mathrm{~Hz}, 2 \mathrm{H}) ;{ }^{13} \mathrm{C}-\mathrm{NMR}$ $\left(101 \mathrm{MHz}, \mathrm{CDCl}_{3}\right) \delta 167.0,143.1,141.5,136.2,134.8,130.2,129.4,128.7,128.5,128.30$, 128.27, 126.8, 119.1, 66.5, 56.4, 14.1; IR (neat) 3421, $1698 \mathrm{~cm}^{-1}$; HRMS (DART) $\mathrm{m} / z$ : $[\mathrm{M}+\mathrm{H}]^{+}$Calcd for $\mathrm{C}_{19} \mathrm{H}_{19} \mathrm{O}_{3} 295.1334$; Found 295.1317.

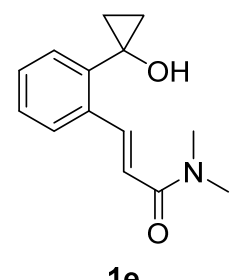

This compound was purified by silica gel column chromatography (Hexane/EtOAc $=5: 1)$. Analytical data for 1e: white solid (mp 111.5-112.9 $\left.{ }^{\circ} \mathrm{C}\right) ;{ }^{1} \mathrm{H}-\mathrm{NMR}\left(400 \mathrm{MHz}, \mathrm{CDCl}_{3}\right) \delta 8.33$ $(\mathrm{d}, J=15.6 \mathrm{~Hz}, 1 \mathrm{H}), 7.56-7.61(\mathrm{~m}, 1 \mathrm{H}), 7.48-7.43(\mathrm{~m}, 1 \mathrm{H}), 7.33-7.28(\mathrm{~m}, 2 \mathrm{H}), 6.85(\mathrm{~d}, J=$ $15.6 \mathrm{~Hz}, 1 \mathrm{H}), 4.23(\mathrm{~s}, 1 \mathrm{H}), 3.17(\mathrm{~s}, 3 \mathrm{H}), 3.05(\mathrm{~s}, 3 \mathrm{H}), 1.25(\mathrm{dd}, J=7.2,5.2 \mathrm{~Hz}, 2 \mathrm{H}), 0.92$ (dd, $J=7.2,5.2 \mathrm{~Hz}, 2 \mathrm{H}) ;{ }^{13} \mathrm{C}-\mathrm{NMR}\left(101 \mathrm{MHz}, \mathrm{CHCl}_{3}\right) \delta 167.3,141.6,140.3,135.6,129.8,129.4$, 128.1, 126.6, 118.9, 55.9, 37.6, 36.0, 14.3; IR (KBr) 3347, $1644 \mathrm{~cm}^{-1}$; HRMS (DART) $m / z:[\mathrm{M}+\mathrm{H}]^{+}$Calcd for $\mathrm{C}_{14} \mathrm{H}_{18} \mathrm{NO}_{2}$ 232.1338; Found 232.1312. 


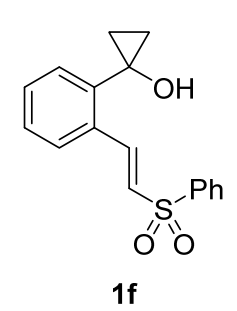

This compound was purified by silica gel column chromatography $($ Hexane/EtOAc $=10: 1)$. Analytical data for 1f: pale-yellow oil; ${ }^{1} \mathrm{H}-\mathrm{NMR}\left(400 \mathrm{MHz}, \mathrm{CDCl}_{3}\right) \delta 8.42(\mathrm{~d}, J=15.6 \mathrm{~Hz}$, $1 \mathrm{H}), 7.96(\mathrm{dt}, J=7.6,1.6 \mathrm{~Hz}, 2 \mathrm{H}), 7.63-7.58(\mathrm{~m}, 1 \mathrm{H}), 7.55-7.51(\mathrm{~m}, 2 \mathrm{H}), 7.49$ (dd, $J=7.6$, $1.6 \mathrm{~Hz}, 1 \mathrm{H}), 7.42(\mathrm{dd}, J=7.6,1.6 \mathrm{~Hz}, 1 \mathrm{H}), 7.35(\mathrm{dd}, J=7.6,1.6 \mathrm{~Hz}, 1 \mathrm{H}), 7.29$ (td, $J=7.6,1.6$ $\mathrm{Hz}, 1 \mathrm{H}), 6.87$ (d, $J=15.6 \mathrm{~Hz}, 1 \mathrm{H}), 2.69(\mathrm{~s}, 1 \mathrm{H}), 1.22(\mathrm{dd}, J=7.2,5.2 \mathrm{~Hz}, 2 \mathrm{H}), 0.90$ (dd, $J=$

7.2, $5.2 \mathrm{~Hz}, 2 \mathrm{H}) ;{ }^{13} \mathrm{C}-\mathrm{NMR}\left(101 \mathrm{MHz}, \mathrm{CHCl}_{3}\right) \delta 141.8,141.1,140.7,133.4,133.2,130.9$, 129.4, 128.8, 128.6, 127.8, 127.5, 56.3, 14.1, One Csp $p^{2}$ signal is missing due to overlapping.; IR (neat) 3464 , $1304 \mathrm{~cm}^{-1}$; HRMS (DART) $m / z$ : $\left[\mathrm{M}+\mathrm{NH}_{4}\right]^{+}$Calcd for $\mathrm{C}_{17} \mathrm{H}_{20} \mathrm{NO}_{3} \mathrm{~S} 318.1164$; Found 318.1174 .

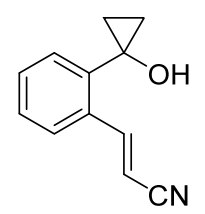

This compound was purified by silica gel column chromatography (Hexane/EtOAc $=5: 1$ ). Analytical data for 1g: pale-yellow oil; ${ }^{1} \mathrm{H}-\mathrm{NMR}\left(400 \mathrm{MHz}, \mathrm{CDCl}_{3}\right) \delta 8.25(\mathrm{~d}, J=17.2 \mathrm{~Hz}$, 1H), 7.56 (dd, $J=7.2,2.0 \mathrm{~Hz}, 1 \mathrm{H}), 7.44-7.33$ (m, 3H), 5.92 (d, $J=17.2 \mathrm{~Hz}, 1 \mathrm{H}), 2.24(\mathrm{~s}, 1 \mathrm{H})$, $1 \mathrm{~g}$ $1.25(\mathrm{dd}, J=7.2,5.2 \mathrm{~Hz}, 2 \mathrm{H}), 0.96(\mathrm{dd}, J=7.2,5.2 \mathrm{~Hz}, 2 \mathrm{H}) ;{ }^{13} \mathrm{C}-\mathrm{NMR}\left(101 \mathrm{MHz}, \mathrm{CHCl}_{3}\right) \delta$ $148.8,140.9,134.3,130.9,129.1,128.8,126.2,118.5,97.5,56.5,14.1$; IR (neat) $3412,2220 \mathrm{~cm}^{-}$ ${ }^{1}$; HRMS (DART) $m / z:\left[\mathrm{M}+\mathrm{NH}_{4}\right]^{+}$Calcd for $\mathrm{C}_{12} \mathrm{H}_{15} \mathrm{~N}_{2} \mathrm{O}$ 203.1184; Found 203.1169.

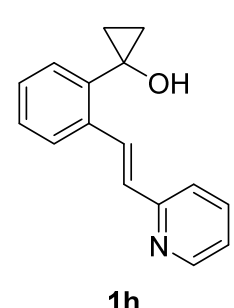

This compound was purified by silica gel column chromatography (Hexane/EtOAc $=5: 1)$. Analytical data for 1h: pale-brown solid (mp 141.9-143.2 $\left.{ }^{\circ} \mathrm{C}\right) ;{ }^{1} \mathrm{H}-\mathrm{NMR}\left(400 \mathrm{MHz}, \mathrm{CDCl}_{3}\right)$ $\delta 8.40(\mathrm{dd}, J=4.8,0.8 \mathrm{~Hz}, 1 \mathrm{H}), 8.26(\mathrm{~d}, J=16.4 \mathrm{~Hz}, 1 \mathrm{H}), 7.67(\mathrm{dd}, J=7.6,0.8 \mathrm{~Hz}, 1 \mathrm{H}), 7.61$ $(\mathrm{td}, J=7.6,1.6 \mathrm{~Hz}, 1 \mathrm{H}), 7.45(\mathrm{~d}, J=7.6 \mathrm{~Hz}, 1 \mathrm{H}), 7.38(\mathrm{dd}, J=7.6,1.6 \mathrm{~Hz}, 1 \mathrm{H}), 7.27(\mathrm{td}, J=$ $7.2,1.2 \mathrm{~Hz}, 1 \mathrm{H}), 7.21(\mathrm{td}, J=7.2,1.2 \mathrm{~Hz}, 1 \mathrm{H}), 7.08(\mathrm{ddd}, J=7.2,4.8,0.8 \mathrm{~Hz}, 1 \mathrm{H}), 7.08(\mathrm{~d}, J$ $=16.4 \mathrm{~Hz}, 1 \mathrm{H}), 5.04(\mathrm{~s}, 1 \mathrm{H}), 1.22(\mathrm{dd}, J=7.2,4.8 \mathrm{~Hz}, 2 \mathrm{H}), 0.92(\mathrm{dd}, J=7.2,4.8 \mathrm{~Hz}, 2 \mathrm{H})$; ${ }^{13} \mathrm{C}-\mathrm{NMR}\left(101 \mathrm{MHz}, \mathrm{CHCl}_{3}\right) \delta 156.1,149.4,140.7,136.8,136.7,130.9,129.4,128.9,128.22,128.18,125.8$, 122.1, 122.0, 56.2, 14.1; IR (KBr) $3158 \mathrm{~cm}^{-1}$; HRMS (ESI) $\mathrm{m} / z$ : $[2 \mathrm{M}+\mathrm{Na}]^{+}$Calcd for $\mathrm{C}_{32} \mathrm{H}_{30} \mathrm{~N}_{2} \mathrm{O}_{2} \mathrm{Na}$ 497.2205; Found 497.2213.

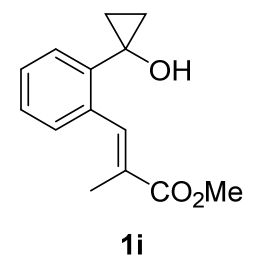

This compound was purified by silica gel column chromatography $($ Hexane/EtOAc $=10: 1)$. Analytical data for 1i: brown oil; ${ }^{1} \mathrm{H}-\mathrm{NMR}\left(400 \mathrm{MHz}, \mathrm{CDCl}_{3}\right) \delta 8.20$ (q, $\left.J=1.2 \mathrm{~Hz}, 1 \mathrm{H}\right)$, $7.42(\mathrm{td}, J=6.0,1.6 \mathrm{~Hz}, 1 \mathrm{H}), 7.34-7.26(\mathrm{~m}, 3 \mathrm{H}), 3.82(\mathrm{~s}, 3 \mathrm{H}), 2.62(\mathrm{~s}, 1 \mathrm{H}), 2.01(\mathrm{~d}, J=1.2$ $\mathrm{Hz}, 3 \mathrm{H}), 1.16(\mathrm{dd}, J=7.2,5.2 \mathrm{~Hz}, 2 \mathrm{H}), 0.91$ (dd, $J=7.2,5.2 \mathrm{~Hz}, 2 \mathrm{H}) ;{ }^{13} \mathrm{C}-\mathrm{NMR}(101 \mathrm{MHz}$, $\left.\mathrm{CHCl}_{3}\right) \delta 169.1,140.9,138.1,136.4,129.7,129.0,128.7,128.3,127.8,56.8,52.2,14.2,14.1$; IR (neat) 3431, $1708 \mathrm{~cm}^{-1}$; HRMS (DART) m/z: [M+ H] Calcd for $\mathrm{C}_{14} \mathrm{H}_{17} \mathrm{O}_{3} 233.1178$; Found 233.1148. 


\section{Synthesis and characterization of $\mathbf{1} \mathbf{j}$}
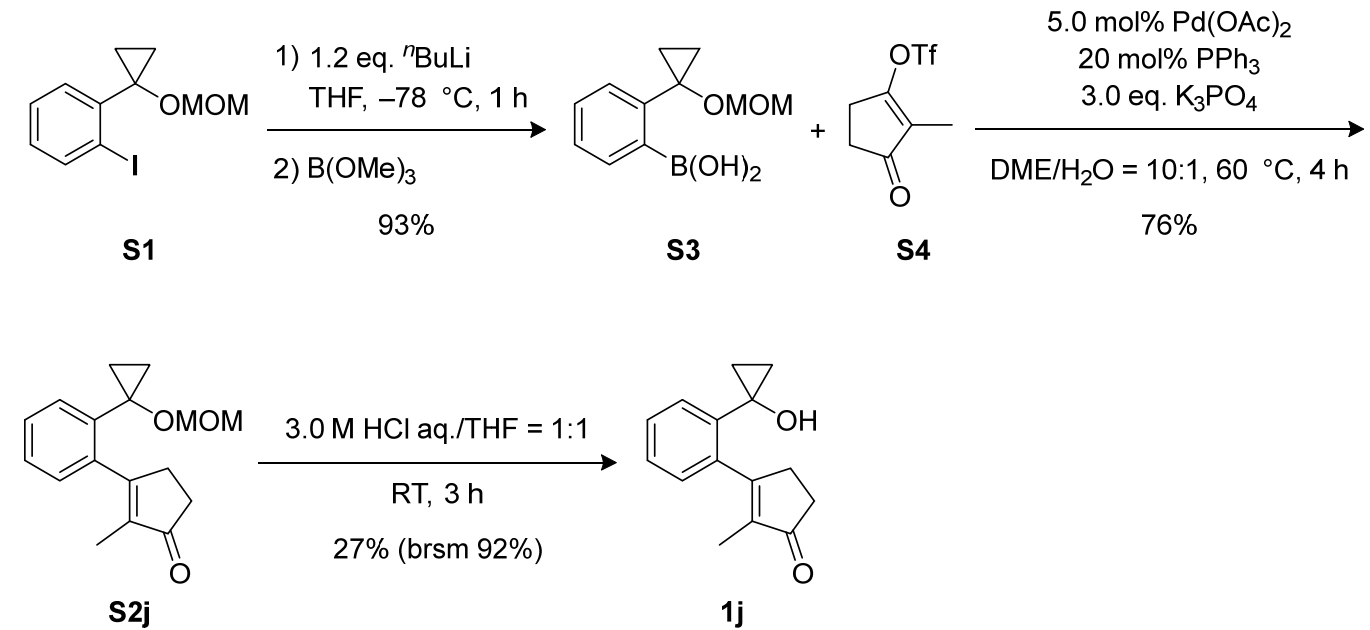

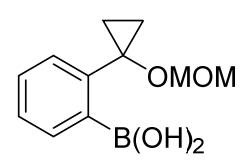

S3

A solution of $\mathbf{S 1}(608 \mathrm{mg}, 2.00 \mathrm{mmol})$ in THF $(10 \mathrm{~mL})$ was cooled to $-78{ }^{\circ} \mathrm{C}$ under $\mathrm{Ar}$ atmosphere. A solution of ${ }^{n} \mathrm{BuLi}(1.50 \mathrm{~mL}, 1.60 \mathrm{M}$ in hexane) was added and the mixture was stirred for $1 \mathrm{~h} . \mathrm{B}(\mathrm{OMe})_{3}(340 \mu \mathrm{L}, 3.05 \mathrm{mmol})$ was then added to the mixture. After being stirred at room temperature for $1 \mathrm{~h}$, the reaction mixture was quenched with water and extracted with $\mathrm{Et}_{2} \mathrm{O}$. The organic layer was washed with brine, dried over $\mathrm{MgSO}_{4}$, and concentrated in vacuo. The residue was purified by silica gel column chromatography (Hexane/EtOAc $=10: 1)$ to furnish $\mathbf{S 3}$ (412 $\mathrm{mg}$, 93\%). Analytical data for S3: pale-yellow oil; ${ }^{1} \mathrm{H}-\mathrm{NMR}\left(400 \mathrm{MHz}, \mathrm{CDCl}_{3}\right) \delta 7.90(\mathrm{dd}, J=8.0,1.6 \mathrm{~Hz}, 1 \mathrm{H})$, $7.42-7.32(\mathrm{~m}, 3 \mathrm{H}), 6.75(\mathrm{~s}, 2 \mathrm{H}), 4.65(\mathrm{~s}, 2 \mathrm{H}), 3.21(\mathrm{~s}, 3 \mathrm{H}), 1.30$ (dd, J=7.2, 5.2 Hz, 2H), 1.02 (dd, $J=7.2$, $5.2 \mathrm{~Hz}, 2 \mathrm{H}) ;{ }^{13} \mathrm{C}-\mathrm{NMR}\left(101 \mathrm{MHz}, \mathrm{CDCl}_{3}\right) \delta$ 143.4, 136.1, 130.2, 129.4, 128.1, 94.8, 63.3, 56.2, 12.8 (One peak derived from an aromatic carbon is obscure due to influence of the boron atom adjacent to that aromatic carbon.); IR (neat) $3398 \mathrm{~cm}^{-1}$; HRMS (DART) $\mathrm{m} / z$ : $\left[\mathrm{M}+\mathrm{NH}_{4}\right]^{+}$Calcd for $\mathrm{C}_{11} \mathrm{H}_{19} \mathrm{BNO}_{4}$ 240.1407; Found 240.1392 .

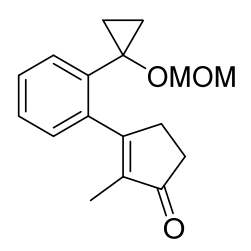

S2j

To a solution of $\mathbf{S 3}$ (412 $\mathrm{mg}, 2.00 \mathrm{mmol})$ and $\mathbf{S 4}{ }^{8}(488 \mathrm{mg}, 2.00 \mathrm{mmol})$ in DME (12 mL) and $\mathrm{H}_{2} \mathrm{O}(1.2 \mathrm{~mL})$ was added $\mathrm{Pd}(\mathrm{OAc})_{2}(25.2 \mathrm{mg}, 0.112 \mathrm{mmol}), \mathrm{PPh}_{3}(106 \mathrm{mg}, 0.404 \mathrm{mmol})$ and $\mathrm{K}_{3} \mathrm{PO}_{4}(1.27 \mathrm{~g}, 6.08 \mathrm{mmol})$. Then the resulting solution was stirred at $60{ }^{\circ} \mathrm{C}$ for $4 \mathrm{~h}$ using an oil bath. After that, the reaction mixture was diluted with water and extracted with $\mathrm{Et}_{2} \mathrm{O}$. The organic layer was washed with brine, dried over $\mathrm{MgSO}_{4}$, and concentrated in vacuo. The residue was purified by silica gel column chromatography (Hexane/EtOAc $=5: 1)$ to furnish $\mathbf{S 2 \mathbf { j }}(416 \mathrm{mg}$, 76\%). Analytical data for S2j: pale-yellow solid (mp 82.9-84.1 $\left.{ }^{\circ} \mathrm{C}\right) ;{ }^{1} \mathrm{H}-\mathrm{NMR}\left(400 \mathrm{MHz}, \mathrm{CDCl}_{3}\right) \delta 7.47-$ $7.45(\mathrm{~m}, 1 \mathrm{H}), 7.38-7.31(\mathrm{~m}, 2 \mathrm{H}), 7.08-7.06(\mathrm{~m}, 1 \mathrm{H}), 4.56(\mathrm{~s}, 2 \mathrm{H}), 3.21(\mathrm{~s}, 3 \mathrm{H}), 3.06-3.03(\mathrm{~m}, 2 \mathrm{H}), 2.57-2.55$ $(\mathrm{m}, 2 \mathrm{H}), 1.61(\mathrm{t}, J=2.0 \mathrm{~Hz}, 3 \mathrm{H}), 1.14(\mathrm{dd}, J=7.2,5.6 \mathrm{~Hz}, 2 \mathrm{H}), 0.87(\mathrm{dd}, J=7.2,5.6 \mathrm{~Hz}, 2 \mathrm{H}) ;{ }^{13} \mathrm{C}-\mathrm{NMR}(101$ $\left.\mathrm{MHz}, \mathrm{CHCl}_{3}\right) \delta 210.3,172.4,139.1,137.4,136.5,130.5,128.34,128.26,128.1,94.8,60.8,55.9,34.7,32.1$, 12.9, 9.3; IR (KBr) 1688, $1156 \mathrm{~cm}^{-1}$; HRMS (DART) $\mathrm{m} / \mathrm{z}$ : $[\mathrm{M}+\mathrm{H}]^{+}$Calcd for $\mathrm{C}_{17} \mathrm{H}_{21} \mathrm{O}_{3}$ 273.1491; Found 273.1465 . 


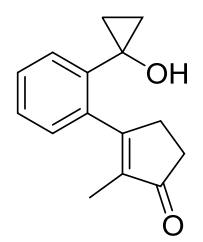

$1 \mathrm{j}$

To a THF solution $(5.0 \mathrm{~mL})$ of $\mathbf{S 2} \mathbf{j}(416 \mathrm{mg}, 1.52 \mathrm{mmol})$ was added aq. HCl (5.0 mL, $3.0 \mathrm{M})$. The resulting mixture was stirred at room temperature overnight. The reaction was quenched with sat. aq. $\mathrm{NaHCO}_{3}$. The aqueous layer was extracted with $\mathrm{Et}_{2} \mathrm{O}$. The combined organic layer was washed with brine, dried over $\mathrm{MgSO}_{4}$, and concentrated in vacuo. The residue was purified by a silica gel column chromatography (Hexane/EtOAc = 3:1) to furnish $\mathbf{1 j}(93.6 \mathrm{mg}, 27 \%)$. Analytical data for $\mathbf{1 j}$ : colorless solid (mp 103.1-103.9 $\left.{ }^{\circ} \mathrm{C}\right) ;{ }^{1} \mathrm{H}-\mathrm{NMR}\left(400 \mathrm{MHz}, \mathrm{CDCl}_{3}\right) \delta 7.49-7.48(\mathrm{~m}$, 1H), 7.37-7.35 (m, 2H), 7.08-7.06 (m, 1H), 3.05-3.02 (m, 2H), 2.57-2.55 (m, 2H), $2.42(\mathrm{~s}, 1 \mathrm{H}), 1.63(\mathrm{t}, J=$ $2.0 \mathrm{~Hz}, 3 \mathrm{H}), 1.07(\mathrm{dd}, J=7.2,5.2 \mathrm{~Hz}, 2 \mathrm{H}), 0.88(\mathrm{dd}, J=7.2,5.2 \mathrm{~Hz}, 2 \mathrm{H}) ;{ }^{13} \mathrm{C}-\mathrm{NMR}\left(101 \mathrm{MHz}^{\mathrm{CDCl}}\right)_{3} \delta$ 210.5, 172.6, 139.1, 138.4, 137.7, 129.4, 128.6, 128.2, 128.1, 57.0, 34.7, 32.6, 14.7, 9.3; IR (KBr) 3468, 1693 $\mathrm{cm}^{-1}$; HRMS (DART) $\mathrm{m} / z$ : [M + H $]^{+}$Calcd for $\mathrm{C}_{15} \mathrm{H}_{17} \mathrm{O}_{2} 229.1229$; Found 229.1202.

\section{Synthesis and characterization of $1 \mathrm{k}$}

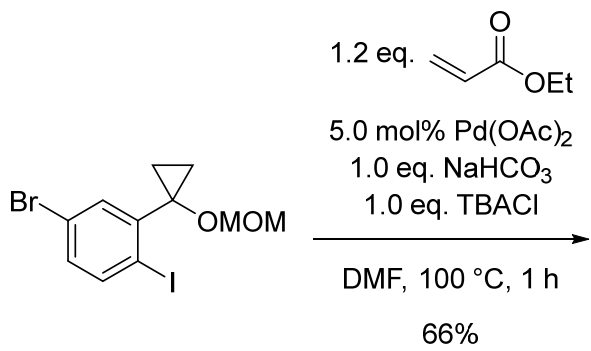

S5

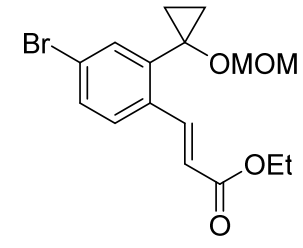

S2k

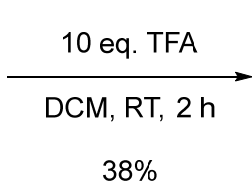

$38 \%$

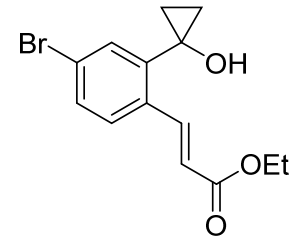

$1 \mathrm{k}$

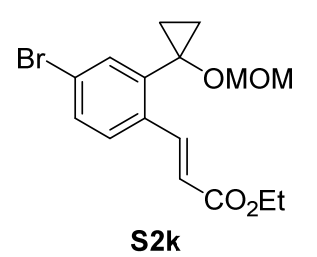

This compound was prepared in the same manner as described for $\mathbf{S 2 a}$ using $\mathbf{S 5}^{4}$ instead of S1 in 66\% yield. This compound was purified by silica gel column chromatography (Hexane/EtOAc $=20: 1)$ and obtained as a mixture with a trace amount of $(Z)$-isomer.

Analytical data for S2k: yellow oil; ${ }^{1} \mathrm{H}-\mathrm{NMR}\left(400 \mathrm{MHz}, \mathrm{CDCl}_{3}\right) \delta 8.40(\mathrm{~d}, J=16.0 \mathrm{~Hz}$, 1H), 7.54-7.43 (m, 3H), $6.41(\mathrm{~d}, J=16.0 \mathrm{~Hz}, 1 \mathrm{H}), 4.56(\mathrm{~s}, 2 \mathrm{H}), 4.28(\mathrm{q}, J=7.2 \mathrm{~Hz}, 2 \mathrm{H})$, $3.13(\mathrm{~s}, 3 \mathrm{H}), 1.34$ (t, $J=7.2 \mathrm{~Hz}, 3 \mathrm{H}), 1.31(\mathrm{dd}, J=7.2,5.6 \mathrm{~Hz}, 2 \mathrm{H}), 0.94(\mathrm{dd}, J=7.2,5.6 \mathrm{~Hz}, 2 \mathrm{H}) ;{ }^{13} \mathrm{C}-\mathrm{NMR}$ $\left(101 \mathrm{MHz}, \mathrm{CDCl}_{3}\right) \delta 166.8,141.6,141.5,134.4,133.1,131.5,128.1,123.5,119.8,95.1,60.6,60.3,55.7,14.4$, 12.7; IR (neat) 1714, $1177 \mathrm{~cm}^{-1}$; HRMS (DART) $\mathrm{m} / z$ : $[\mathrm{M}+\mathrm{H}]^{+}$Calcd for $\mathrm{C}_{16} \mathrm{H}_{20} \mathrm{BrO}_{4}$ 355.0545; Found 355.0570 .

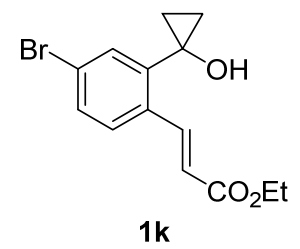

This compound was prepared in the same manner as described for 1a using S2k instead of S2a in 38\% yield. This compound was purified by silica gel column chromatography (Hexane/EtOAc = 10:1). Analytical data for S2k: pale-yellow oil; ${ }^{1} \mathrm{H}-\mathrm{NMR}(400 \mathrm{MHz}$, $\left.\mathrm{CDCl}_{3}\right) \delta 8.34(\mathrm{~d}, J=16.0 \mathrm{~Hz}, 1 \mathrm{H}), 7.56(\mathrm{~d}, J=1.2 \mathrm{~Hz}, 1 \mathrm{H}), 7.48(\mathrm{~d}, J=8.4 \mathrm{~Hz}, 1 \mathrm{H}), 7.44$ $(\mathrm{d}, J=8.4 \mathrm{~Hz}, 1 \mathrm{H}), 6.39(\mathrm{~d}, J=16.0 \mathrm{~Hz}, 1 \mathrm{H}), 4.25$ (q, $J=7.2 \mathrm{~Hz}, 2 \mathrm{H}), 3.17(\mathrm{~s}, 1 \mathrm{H}), 1.33$ $(\mathrm{t}, J=7.2 \mathrm{~Hz}, 3 \mathrm{H}), 1.25(\mathrm{dd}, J=7.2,5.2 \mathrm{~Hz}, 2 \mathrm{H}), 0.94(\mathrm{dd}, J=7.2,5.2 \mathrm{~Hz}, 2 \mathrm{H}) ;{ }^{13} \mathrm{C}-\mathrm{NMR}\left(101 \mathrm{MHz}, \mathrm{CDCl}_{3}\right)$ $\delta 167.1,143.3,141.4,133.9,132.5,131.5,128.3,123.9,120.0,60.8,56.0,14.4,14.2$; IR (neat) 3414,1711 $\mathrm{cm}^{-1}$; HRMS (DART) $\mathrm{m} / z$ : $[\mathrm{M}+\mathrm{H}]^{+}$Calcd for $\mathrm{C}_{14} \mathrm{H}_{19} \mathrm{NBrO}_{3} 328.0548$; Found 328.0538. 


\section{Synthesis and characterization of 11}

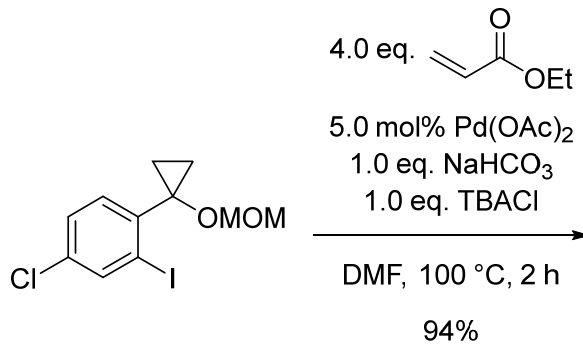

S6

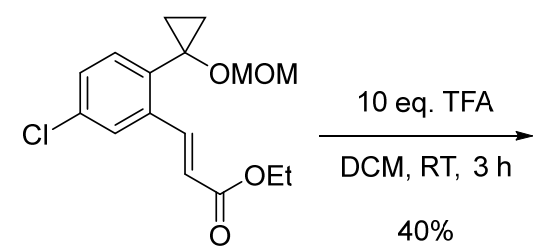

S21<smiles>CCOC(=O)/C=C/c1cc(Cl)ccc1C1(O)CC1</smiles>

11<smiles>CCOC(=O)/C=C/c1cc(Cl)ccc1C1(OOC)CC1</smiles>

S2I

This compound was prepared in the same manner as described for S2a using $\mathbf{S 6}^{4}$ instead of $\mathbf{S 1}$ in $94 \%$ yield. This compound was purified by silica gel column chromatography $($ Hexane/EtOAc $=20: 1)$. Analytical data for S2I: yellow oil; ${ }^{1} \mathrm{H}-\mathrm{NMR}(400 \mathrm{MHz}$, $\left.\mathrm{CDCl}_{3}\right) \delta 8.41(\mathrm{~d}, J=16.0 \mathrm{~Hz}, 1 \mathrm{H}), 7.60(\mathrm{~d}, J=1.6 \mathrm{~Hz}, 1 \mathrm{H}), 7.33(\mathrm{~d}, J=8.4 \mathrm{~Hz}, 1 \mathrm{H})$, $7.28-7.26(\mathrm{~m}, 1 \mathrm{H}), 6.42(\mathrm{~d}, J=16.0 \mathrm{~Hz}, 1 \mathrm{H}), 4.55(\mathrm{~s}, 2 \mathrm{H}), 4.28(\mathrm{q}, J=7.2 \mathrm{~Hz}, 2 \mathrm{H}), 3.12(\mathrm{~s}, 3 \mathrm{H}), 1.35(\mathrm{t}, J=$ $7.2 \mathrm{~Hz}, 3 \mathrm{H}), 1.30(\mathrm{dd}, J=7.2,5.6 \mathrm{~Hz}, 2 \mathrm{H}), 0.91(\mathrm{dd}, J=7.2,5.6 \mathrm{~Hz}, 2 \mathrm{H}) ;{ }^{13} \mathrm{C}-\mathrm{NMR}\left(101 \mathrm{MHz}, \mathrm{CDCl}_{3}\right) \delta$ 166.6, 141.3, 138.1, 137.2, 134.3, 131.6, 129.2, 126.5, 120.5, 95.0, 60.6, 60.0, 55.6, 14.3, 12.6; IR (neat) 1715, $1179 \mathrm{~cm}^{-1}$; HRMS (DART) $\mathrm{m} / z$ : $[\mathrm{M}+\mathrm{H}]^{+}$Calcd for $\mathrm{C}_{16} \mathrm{H}_{20} \mathrm{ClO}_{4} 313.1021$; Found 313.1008.

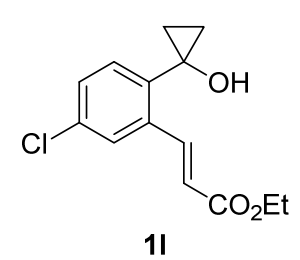

This compound was prepared in the same manner as described for 1a using S2I instead of S2a in $40 \%$ yield. This compound was purified by silica gel column chromatography $($ Hexane/EtOAc $=10: 1)$. Analytical data for 1l: yellow oil; ${ }^{1} \mathrm{H}-\mathrm{NMR}\left(400 \mathrm{MHz}, \mathrm{CDCl}_{3}\right)$ $\delta 8.35(\mathrm{~d}, J=16.0 \mathrm{~Hz}, 1 \mathrm{H}), 7.57(\mathrm{~d}, J=2.0 \mathrm{~Hz}, 1 \mathrm{H}), 7.35(\mathrm{~d}, J=8.0 \mathrm{~Hz}, 1 \mathrm{H}), 7.28-7.26$ $(\mathrm{m}, 1 \mathrm{H}), 6.39(\mathrm{~d}, J=16.0 \mathrm{~Hz}, 1 \mathrm{H}), 4.25(\mathrm{q}, J=7.2 \mathrm{~Hz}, 2 \mathrm{H}), 3.24(\mathrm{~s}, 1 \mathrm{H}), 1.33(\mathrm{t}, J=7.2$ $\mathrm{Hz}, 3 \mathrm{H}), 1.23(\mathrm{dd}, J=7.2,5.2 \mathrm{~Hz}, 2 \mathrm{H}), 0.90(\mathrm{dd}, J=7.2,5.2 \mathrm{~Hz}, 2 \mathrm{H}) ;{ }^{13} \mathrm{C}-\mathrm{NMR}\left(101 \mathrm{MHz}, \mathrm{CDCl}_{3}\right) \delta 166.9$, 141.2, 139.9, 136.6, 134.3, 130.8, 129.7, 126.6, 120.6, 60.9, 55.7, 14.3, 14.1; IR (neat) 3414, $1712 \mathrm{~cm}^{-1}$; HRMS (DART) $\mathrm{m} / \mathrm{z}:[\mathrm{M}+\mathrm{H}]^{+}$Calcd for $\mathrm{C}_{14} \mathrm{H}_{19} \mathrm{ClNO}_{3} 284.1054$; Found 284.1076. 


\section{Synthesis and characterization of $1 \mathrm{~m}$}
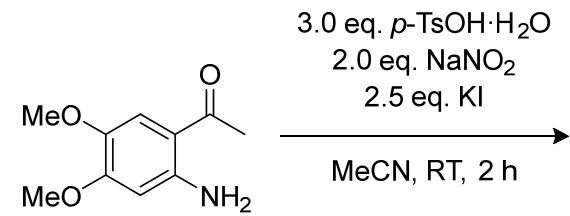

$92 \%$

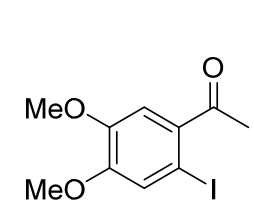

S7

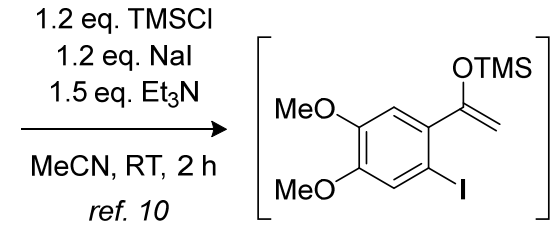

S8

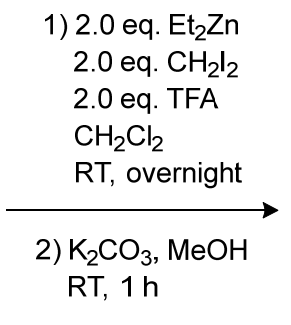

$72 \%$ (2 steps)<smiles></smiles>

S11

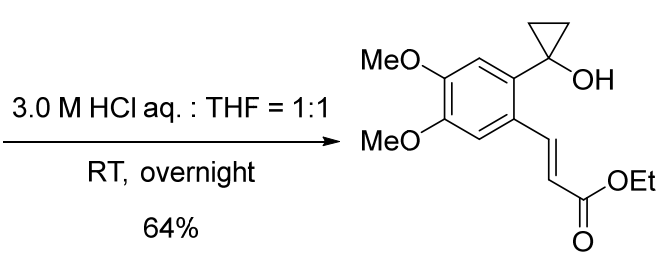

$1 \mathrm{~m}$

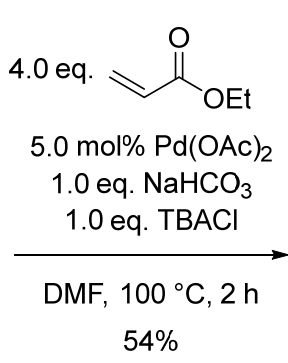

S10<smiles>COc1cc(I)c(C2(O)CC2)cc1OC</smiles>

S9

To a $200 \mathrm{~mL}$ two-necked flask was charged with $\mathbf{S} 7^{9}(4.21 \mathrm{~g}, 13.8 \mathrm{mmol})$ and $\mathrm{NaI}$ ( $2.48 \mathrm{~g}$, $16.6 \mathrm{mmol})$, followed by dry $\mathrm{MeCN}(40 \mathrm{~mL})$. To the mixture was added $\mathrm{Et}_{3} \mathrm{~N}(2.90 \mathrm{~mL}$, $20.7 \mathrm{mmol})$ and $\mathrm{TMSCl}(2.10 \mathrm{~mL}, 16.6 \mathrm{mmol})$, successively. The resulting solution was stirred at room temperature for $2 \mathrm{~h}$. After that, the reaction mixture was quenched with water and washed with brine. The solution was dried over $\mathrm{MgSO}_{4}$, and concentrated in vacuo. The residue $\mathbf{S 8}$ was used for the next reaction without further purification. To a dried $300 \mathrm{~mL}$ two-necked flask was added degassed dry DCM (100 mL) and $\mathrm{Et}_{2} \mathrm{Zn}(28 \mathrm{~mL}, 1.07 \mathrm{M}$ in hexane) under Ar. The solution was cooled to $10{ }^{\circ} \mathrm{C}$ and a solution of TFA $(2.10 \mathrm{~mL}, 27.6 \mathrm{mmol})$ in degassed dry DCM $(15 \mathrm{~mL})$ was then dripped into the reaction mixture for $1 \mathrm{~h}$ (very slowly). On stirring for additional $1 \mathrm{~h}$, a solution of $\mathrm{CH}_{2} \mathrm{I}_{2}(2.40 \mathrm{~mL}, 27.6 \mathrm{mmol})$ in degassed dry DCM $(20 \mathrm{~mL})$ was added to the reaction mixture for $1 \mathrm{~h}$ (very slowly). After stirring at $\mathrm{rt}$ for $3 \mathrm{~h}, \mathbf{S 8}$ was added to the mixture. The reaction mixture was stirred at $\mathrm{rt}$ overnight. The reaction was quenched with sat. aq. $\mathrm{NH}_{4} \mathrm{Cl}$ and extracted with EtOAc. The organic layer was washed with sat. aq. $\mathrm{NaHCO}_{3}$, dried over $\mathrm{MgSO}_{4}$, and concentrated in vacuo. To a $\mathrm{MeOH}$ solution $(40 \mathrm{~mL})$ of the crude material in a $200 \mathrm{~mL}$ flask was added $\mathrm{K}_{2} \mathrm{CO}_{3}(2.01 \mathrm{mg}, 14.5 \mathrm{mmol})$ at $\mathrm{rt}$. After being stirred for $1 \mathrm{~h}$, the reaction mixture was diluted with water and extracted with EtOAc. The organic layer was washed with brine, dried over $\mathrm{MgSO}_{4}$, and concentrated. The residue was purified by a silica gel column chromatography $($ Hexane/EtOAc $=20: 1)$ to furnish S9 (3.17 g, 72\%, 2 steps from S7). Analytical data for S9: pale-yellow solid (mp 121.5-122.8 ${ }^{\circ} \mathrm{C}$ ); ${ }^{1} \mathrm{H}-\mathrm{NMR}\left(400 \mathrm{MHz}, \mathrm{CDCl}_{3}\right) \delta 7.26(\mathrm{~s}, 1 \mathrm{H}), 6.92(\mathrm{~s}, 1 \mathrm{H}), 3.87(\mathrm{~s}, 3 \mathrm{H}), 3.86(\mathrm{~s}, 3 \mathrm{H}), 2.91(\mathrm{~s}, 1 \mathrm{H}), 1.29(\mathrm{dd}, J=$ 
7.2, $5.2 \mathrm{~Hz}, 2 \mathrm{H}), 0.97(\mathrm{dd}, J=7.2,5.2 \mathrm{~Hz}, 2 \mathrm{H}) ;{ }^{13} \mathrm{C}-\mathrm{NMR}\left(101 \mathrm{MHz}, \mathrm{CHCl}_{3}\right) \delta 149.3,149.0,136.6,121.8$, 114.2, 88.8, 61.1, 56.3, 56.1, 16.2; IR (KBr) $3476 \mathrm{~cm}^{-1}$; HRMS (DART) $m / z:\left[\mathrm{M}+\mathrm{NH}_{4}\right]^{+} \mathrm{Calcd}$ for $\mathrm{C}_{11} \mathrm{H}_{17} \mathrm{IN}$ $\mathrm{O}_{3}$ 338.0253; Found 338.0269.<smiles>COc1cc(I)c(C2(O[SbH3])CC2)cc1OC</smiles>
brine, dried over $\mathrm{MgSO}_{4}$, and concentrated in vacuo. The residue was purified by a silica gel column chromatography (Hexane/EtOAc $=20: 1$ ) to furnish S10 (1.51 g, quant.). Analytical data for S10: white solid (mp 65.4-67.0 $\left.{ }^{\circ} \mathrm{C}\right) ;{ }^{1} \mathrm{H}-\mathrm{NMR}\left(400 \mathrm{MHz}, \mathrm{CDCl}_{3}\right) \delta 7.28(\mathrm{~s}, 1 \mathrm{H}), 6.81(\mathrm{~s}, 1 \mathrm{H}), 3.85(\mathrm{~s}, 3 \mathrm{H}), 3.85(\mathrm{~s}, 3 \mathrm{H}), 1.12$ $(\mathrm{dd}, J=7.1,5.2 \mathrm{~Hz}, 2 \mathrm{H}), 0.88(\mathrm{dd}, J=7.2,5.2 \mathrm{~Hz}, 2 \mathrm{H}), 0.78(\mathrm{~s}, 9 \mathrm{H}),-0.14(\mathrm{~s}, 6 \mathrm{H}) ;{ }^{13} \mathrm{C}-\mathrm{NMR}(101 \mathrm{MHz}$, $\left.\mathrm{CDCl}_{3}\right) \delta 148.8,148.3,136.8,122.5,114.1,90.4,61.5,56.2,56.1,25.7,17.8,15.7,-3.9$; IR (neat) $1252 \mathrm{~cm}^{-1}$; HRMS (DART) $m / z:[\mathrm{M}-\mathrm{TBSO}]^{+}$Calcd for $\mathrm{C}_{11} \mathrm{H}_{13} \mathrm{IO}_{2}$ 302.9882; Found 302.9900.

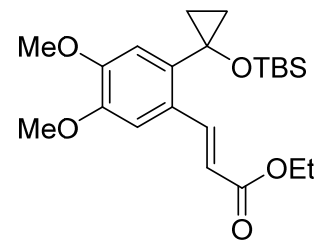

This compound was prepared in the same manner as described for S2a using S10 instead of S1 in 54\% yield. This compound was purified by silica gel column chromatography $($ Hexane/EtOAc $=20: 1)$. Analytical data for S11: white solid $\left(\mathrm{mp} 100.5-102.2{ }^{\circ} \mathrm{C}\right) ;{ }^{1} \mathrm{H}-$ NMR (400 MHz, $\left.\mathrm{CDCl}_{3}\right) \delta 8.52(\mathrm{~d}, J=16.0 \mathrm{~Hz}, 1 \mathrm{H}), 7.12(\mathrm{~s}, 1 \mathrm{H}), 6.84(\mathrm{~s}, 1 \mathrm{H}), 6.31(\mathrm{~d}$, S11 $J=16.0 \mathrm{~Hz}, 1 \mathrm{H}), 4.27$ (q, $J=7.2 \mathrm{~Hz}, 2 \mathrm{H}), 3.91(\mathrm{~s}, 6 \mathrm{H}), 1.33$ (t, $J=7.2 \mathrm{~Hz}, 3 \mathrm{H}), 1.13$ $(\mathrm{dd}, J=7.2,5.2 \mathrm{~Hz}, 2 \mathrm{H}), 0.90(\mathrm{dd}, J=7.2,5.2 \mathrm{~Hz}, 2 \mathrm{H}), 0.75(\mathrm{~s}, 9 \mathrm{H}),-0.17(\mathrm{~s}, 6 \mathrm{H}) ;{ }^{13} \mathrm{C}-\mathrm{NMR}(101 \mathrm{MHz}$, $\left.\mathrm{CDCl}_{3}\right) \delta 167.3,149.8,148.5,143.1,135.8,128.1,116.8,111.6,108.9,60.3,57.0,56.1,56.0,25.5,17.7,14.4$, 14.3, -4.1; IR (neat) 1702, $1252 \mathrm{~cm}^{-1}$; HRMS (DART) $m / z:\left[\mathrm{M}+\mathrm{NH}_{4}\right]^{+}$Calcd for $\mathrm{C}_{22} \mathrm{H}_{38} \mathrm{NO}_{5} \mathrm{Si}_{424.2519}$; Found 424.2539 .

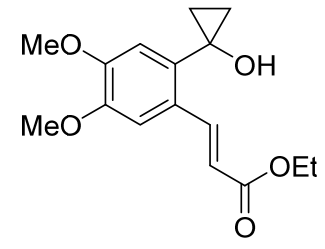

$1 \mathrm{~m}$

To a THF solution $(1.0 \mathrm{~mL})$ of $\mathbf{S 1 1}(81.3 \mathrm{mg}, 0.200 \mathrm{mmol})$ was added aq. $\mathrm{HCl}(1.0 \mathrm{~mL}$, $3 \mathrm{M})$. The resulting mixture was stirred at room temperature overnight. The reaction was quenched with sat. aq. $\mathrm{NaHCO}_{3}$. The aqueous layer was extracted with $\mathrm{Et}_{2} \mathrm{O}$. The combined organic layer was washed with brine, dried over $\mathrm{MgSO}_{4}$, and concentrated in vacuo. The residue was purified by a silica gel column chromatography (Hexane/EtOAc $=5: 1)$ to furnish $\mathbf{1 m}(37.5 \mathrm{mg}, 64 \%)$. Analytical data for $\mathbf{1 m}$ : white solid (mp $\left.142.3-144.4{ }^{\circ} \mathrm{C}\right) ;{ }^{1} \mathrm{H}-\mathrm{NMR}$ $\left(400 \mathrm{MHz} \mathrm{CDCl}_{3}\right) \delta 8.44(\mathrm{~d}, J=16.0 \mathrm{~Hz}, 1 \mathrm{H}), 7.12(\mathrm{~s}, 1 \mathrm{H}), 6.96(\mathrm{~s}, 1 \mathrm{H}), 6.35(\mathrm{~d}, J=16.0 \mathrm{~Hz}, 1 \mathrm{H}), 4.28(\mathrm{q}, J$ $=7.2 \mathrm{~Hz}, 2 \mathrm{H}), 3.93(\mathrm{~s}, 3 \mathrm{H}), 3.92(\mathrm{~s}, 3 \mathrm{H}), 2.24(\mathrm{~s}, 1 \mathrm{H}), 1.35(\mathrm{t}, J=7.2 \mathrm{~Hz}, 3 \mathrm{H}), 1.28(\mathrm{dd}, J=7.2,5.2 \mathrm{~Hz}, 2 \mathrm{H})$, $0.96(\mathrm{dd}, J=7.2,5.2 \mathrm{~Hz}, 2 \mathrm{H}) ;{ }^{13} \mathrm{C}-\mathrm{NMR}\left(101 \mathrm{MHz}, \mathrm{CDCl}_{3}\right) \delta 167.5,150.5,148.9,141.9,135.5,127.0,117.2$, $112.5,108.7,60.6,56.2,56.1,14.6,14.4$, A signal of $\mathrm{OCH}_{3}$ is missing due to overlapping.; IR (neat) 3485, $1686 \mathrm{~cm}^{-1}$; HRMS (DART) m/z: [M+ $\left.\mathrm{NH}_{4}\right]^{+}$Calcd for $\mathrm{C}_{16} \mathrm{H}_{24} \mathrm{NO}_{5}$ 310.1655; Found 310.1682. 


\section{Synthesis and characterization of $1 \mathrm{n}$}
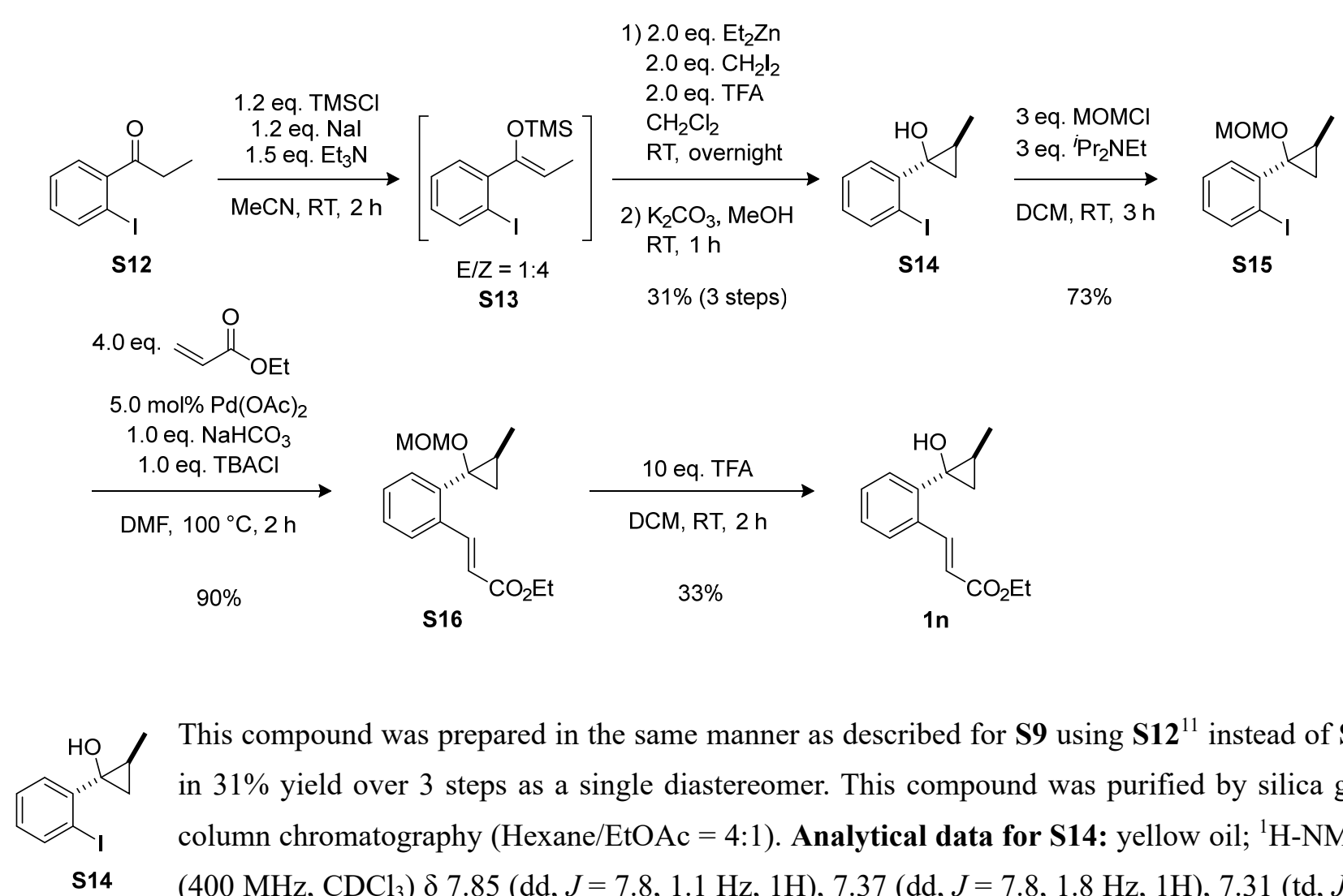

This compound was prepared in the same manner as described for $\mathbf{S 9}$ using $\mathbf{S 1 2}{ }^{11}$ instead of $\mathbf{S 7}$ in $31 \%$ yield over 3 steps as a single diastereomer. This compound was purified by silica gel column chromatography (Hexane/EtOAc $=4: 1)$. Analytical data for S14: yellow oil; ${ }^{1} \mathrm{H}-\mathrm{NMR}$ $\left(400 \mathrm{MHz}, \mathrm{CDCl}_{3}\right) \delta 7.85(\mathrm{dd}, J=7.8,1.1 \mathrm{~Hz}, 1 \mathrm{H}), 7.37(\mathrm{dd}, J=7.8,1.8 \mathrm{~Hz}, 1 \mathrm{H}), 7.31(\mathrm{td}, J=$ 7.8, $1.1 \mathrm{~Hz}, 1 \mathrm{H}), 6.99(\mathrm{td}, J=7.8,1.8 \mathrm{~Hz}, 1 \mathrm{H}), 2.68(\mathrm{~s}, 1 \mathrm{H}), 1.43(\mathrm{~d}, J=6.0 \mathrm{~Hz}, 3 \mathrm{H}), 1.23-1.19(\mathrm{~m}, 1 \mathrm{H}), 1.12-$ $1.03(\mathrm{~m}, 1 \mathrm{H}), 0.83-0.80(\mathrm{~m}, 1 \mathrm{H}) ;{ }^{13} \mathrm{C}-\mathrm{NMR}\left(101 \mathrm{MHz}, \mathrm{CDCl}_{3}\right) \delta 145.6,139.5,130.9,129.5,128.2,100.3$, 63.3, 20.3, 20.1, 12.9; IR (neat) $3443 \mathrm{~cm}^{-1}$; HRMS (DART) $\mathrm{m} / z$ : $\left[\mathrm{M}+\mathrm{NH}_{4}\right]^{+}$Calcd for $\mathrm{C}_{10} \mathrm{H}_{15}{ }^{127} \mathrm{INO} 292.0198$; Found 292.0173.

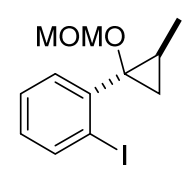

S15 To a solution of $\mathbf{S 1 4}(432 \mathrm{mg}, 1.6 \mathrm{mmol})$ and ${ }^{i} \operatorname{Pr}_{2} \operatorname{NEt}(0.82 \mathrm{~mL}, 4.8 \mathrm{mmol})$ in DCM (7 mL) was added $\mathrm{MOMCl}(0.36 \mathrm{~mL}, 4.5 \mathrm{mmol})$ at $0{ }^{\circ} \mathrm{C}$. After being stirred at $\mathrm{rt}$ for $3 \mathrm{~h}$, the reaction was quenched with sat. aq. $\mathrm{NaHCO}_{3}$. The mixture was extracted with $\mathrm{Et}_{2} \mathrm{O}$, washed with brine, and concentrated in vacuo. The residue was purified by silica gel column chromatography (Hexane/EtOAc $=20: 1)$ to afford $\mathbf{S 1 5}\left(371.6 \mathrm{mg}, 73 \%\right.$ yield). Analytical data for S15: yellow oil; ${ }^{1} \mathrm{H}-\mathrm{NMR}$ $\left(400 \mathrm{MHz}, \mathrm{CDCl}_{3}\right) \delta 7.89(\mathrm{dd}, J=7.8,0.9 \mathrm{~Hz}, 1 \mathrm{H}), 7.34-7.26(\mathrm{~m}, 2 \mathrm{H}), 6.97(\mathrm{td}, J=7.8,2.3 \mathrm{~Hz}, 1 \mathrm{H}), 4.65(\mathrm{~d}$, $J=6.9 \mathrm{~Hz}, 1 \mathrm{H}), 4.63(\mathrm{~d}, J=6.9 \mathrm{~Hz}, 1 \mathrm{H}), 3.16(\mathrm{~s}, 3 \mathrm{H}), 1.45(\mathrm{~d}, J=5.9 \mathrm{~Hz}, 3 \mathrm{H}), 1.25-1.22(\mathrm{~m}, 1 \mathrm{H}), 1.10-1.01$ $(\mathrm{m}, 1 \mathrm{H}), 0.88-0.85(\mathrm{~m}, 1 \mathrm{H}) ;{ }^{13} \mathrm{C}-\mathrm{NMR}\left(101 \mathrm{MHz}, \mathrm{CDCl}_{3}\right) \delta 143.8,139.9,131.9,129.3,127.5,101.0,95.4$, 67.6, 55.8, 20.5, 18.4, 13.5; IR (neat) $1153 \mathrm{~cm}^{-1}$; HRMS (DART) $\mathrm{m} / z$ : $\left[\mathrm{M}+\mathrm{NH}_{4}\right]^{+}$Calcd for $\mathrm{C}_{12} \mathrm{H}_{19}{ }^{127} \mathrm{INO}_{2}$ 336.0461 ; Found 336.0474 . 
mомо This compound was prepared in the same manner as described for S2a using $\mathbf{S 1 5}$ instead of $\mathbf{S 1}$ in $90 \%$ yield (ca. $6 \%$ of a stereoisomer was contained). This compound was purified by silica gel column chromatography (Hexane/EtOAc $=20: 1$ ). Analytical data for S16: yellow oil; ${ }^{1} \mathrm{H}-\mathrm{NMR}\left(400 \mathrm{MHz}, \mathrm{CDCl}_{3}\right) \delta 8.35(\mathrm{~d}, J=16.0 \mathrm{~Hz}, 1 \mathrm{H}), 7.65-7.63(\mathrm{~m}, 1 \mathrm{H}), 7.37-7.29$ $(\mathrm{m}, 3 \mathrm{H}), 6.40(\mathrm{~d}, J=16.0 \mathrm{~Hz}, 1 \mathrm{H}), 4.63(\mathrm{~d}, J=6.4 \mathrm{~Hz}, 1 \mathrm{H}), 4.57$ (d, $J=6.4 \mathrm{~Hz}, 1 \mathrm{H}), 4.33-$ $4.24(\mathrm{~m}, 2 \mathrm{H}), 3.13(\mathrm{~s}, 3 \mathrm{H}), 1.41(\mathrm{~d}, J=6.4 \mathrm{~Hz}, 3 \mathrm{H}), 1.35$ (t, $J=7.2 \mathrm{~Hz}, 3 \mathrm{H}), 1.23$ (dd, $J=9.6,5.5 \mathrm{~Hz}, 1 \mathrm{H})$, $1.03-0.96(\mathrm{~m}, 1 \mathrm{H}), 0.82-0.78(\mathrm{~m}, 1 \mathrm{H}) ;{ }^{13} \mathrm{C}-\mathrm{NMR}\left(101 \mathrm{MHz}, \mathrm{CDCl}_{3}\right) \delta$ 167.0, 143.0, 141.2, 135.1, 129.9, 129.4, 128.1, 126.3, 118.9, 95.2, 63.2, 60.4, 55.7, 19.9, 16.9, 14.3, 12.6; IR (neat) $1714 \mathrm{~cm}^{-1}$; HRMS (DART) $m / z$ : $\left[\mathrm{M}+\mathrm{NH}_{4}\right]^{+}$Calcd for $\mathrm{C}_{17} \mathrm{H}_{26} \mathrm{NO}_{4} 308.1862$; Found 308.1870 .

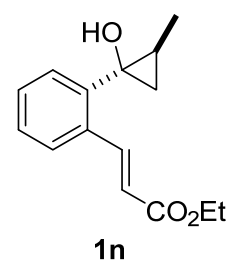

This compound was prepared in the same manner as described for S2a using S15 instead of S1 in $90 \%$ yield. This compound was purified by silica gel column chromatography $(\mathrm{Hexane} / \mathrm{EtOAc}=5: 1)$. Analytical data for $1 \mathrm{n}$ : colorless oil; ${ }^{1} \mathrm{H}-\mathrm{NMR}\left(400 \mathrm{MHz}, \mathrm{CDCl}_{3}\right) \delta$ $8.31(\mathrm{~d}, J=16.0 \mathrm{~Hz}, 1 \mathrm{H}), 7.65-7.63(\mathrm{~m}, 1 \mathrm{H}), 7.41-7.30(\mathrm{~m}, 3 \mathrm{H}), 6.42(\mathrm{~d}, J=16.0 \mathrm{~Hz}, 1 \mathrm{H})$, $4.31-4.25(\mathrm{~m}, 2 \mathrm{H}), 2.08(\mathrm{~s}, 1 \mathrm{H}), 1.43(\mathrm{~d}, J=5.9 \mathrm{~Hz}, 3 \mathrm{H}), 1.35$ (t, $J=7.1 \mathrm{~Hz}, 3 \mathrm{H}), 1.19-1.08$ (m, 2H), 0.75-0.72 (m, 1H); ${ }^{13} \mathrm{C}-\mathrm{NMR}\left(101 \mathrm{MHz}, \mathrm{CDCl}_{3}\right) \delta 166.9,142.7,142.4,134.7,129.9,129.1,128.3$, 126.6, 119.5, 60.5, 58.9, 19.3, 19.3, 14.3, 12.1; IR (neat) 3446, $1698 \mathrm{~cm}^{-1}$; HRMS (DART) $m / z:\left[\mathrm{M}+\mathrm{NH}_{4}\right]^{+}$ Calcd for $\mathrm{C}_{15} \mathrm{H}_{22} \mathrm{NO}_{3} 264.1600$; Found 264.1592.

\section{Synthesis and characterization of 1q}
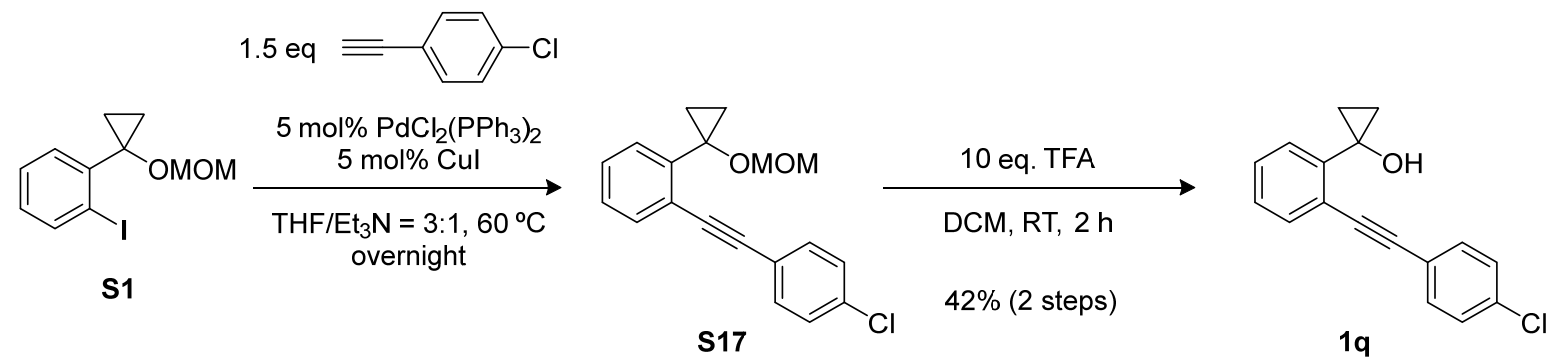

A mixture of $\mathbf{S 1}$ (456 mg, $1.5 \mathrm{mmol}$ ), 4-chlorophenylacetylene (307.3 mg, $2.25 \mathrm{mmol}), \mathrm{PdCl}_{2}\left(\mathrm{PPh}_{3}\right)_{2}(91 \mathrm{mg}$, $0.075 \mathrm{mmol})$, and $\mathrm{CuI}(14.3 \mathrm{mg}, 0.075 \mathrm{mmol})$ in THF $(15 \mathrm{~mL})$ and $\mathrm{Et}_{3} \mathrm{~N}(5 \mathrm{~mL})$ was degassed at $-78^{\circ} \mathrm{C}$, and stirred at $60{ }^{\circ} \mathrm{C}$ using an oil bath. After being stirred overnight, the mixture was concentrated in vacuo. The residue was dissolved in $\mathrm{Et}_{2} \mathrm{O}$ and filtered through celite. The filtrate was concentrated in vacuo. The residue was purified by silica gel column chromatography (Hexane/EtOAc $=20: 1$ ) to afford $\mathbf{S 1 7}$, which still contained a small amount of inseparable impurities. This compound was used for the next step without further purification. To a solution of $\mathbf{S 1 7}$ in degassed DCM $(7 \mathrm{~mL})$ was added TFA $(1 \mathrm{~mL})$. After being stirred at rt for $2 \mathrm{~h}$, the mixture was neutralized with sat. aq. $\mathrm{NaHCO}_{3}$. The mixture was extracted with EtOAc, washed with brine, dried over $\mathrm{MgSO}_{4}$, and concentrated in vacuo. The residue was purified by silica gel column chromatography (Hexane/EtOAc $=10: 1)$ to afford 1 q $(183 \mathrm{mg}, 42 \%$ yield over 2 steps). Analytical data for 1q: pale yellow oil; ${ }^{1} \mathrm{H}-\mathrm{NMR}\left(400 \mathrm{MHz}, \mathrm{CDCl}_{3}\right) \delta$ 7.56-7.54 (m, 1H), 7.48-7.45 (m, 2H), 7.41-7.38 (m, 1H), 
7.36-7.26 (m, 4H), $3.20(\mathrm{~s}, 1 \mathrm{H}), 1.24(\mathrm{dd}, J=7.6,5.3 \mathrm{~Hz}, 2 \mathrm{H}), 1.06(\mathrm{dd}, J=7.6,5.3 \mathrm{~Hz}, 2 \mathrm{H}) ;{ }^{13} \mathrm{C}-\mathrm{NMR}(101$ $\left.\mathrm{MHz}, \mathrm{CDCl}_{3}\right) \delta 144.1,134.7,132.6,132.5,128.8,128.8,128.1,127.8,123.0,121.4,93.4,88.7,57.2,14.3$; IR (neat) $3398 \mathrm{~cm}^{-1}$; HRMS (DART) $\mathrm{m} / z$ : $\left[\mathrm{M}+\mathrm{NH}_{4}\right]^{+}$Calcd for $\mathrm{C}_{17} \mathrm{H}_{17}{ }^{35} \mathrm{ClNO} 286.0999$; Found 286.1004 .

\section{Synthesis and characterization of $1 r-w$}
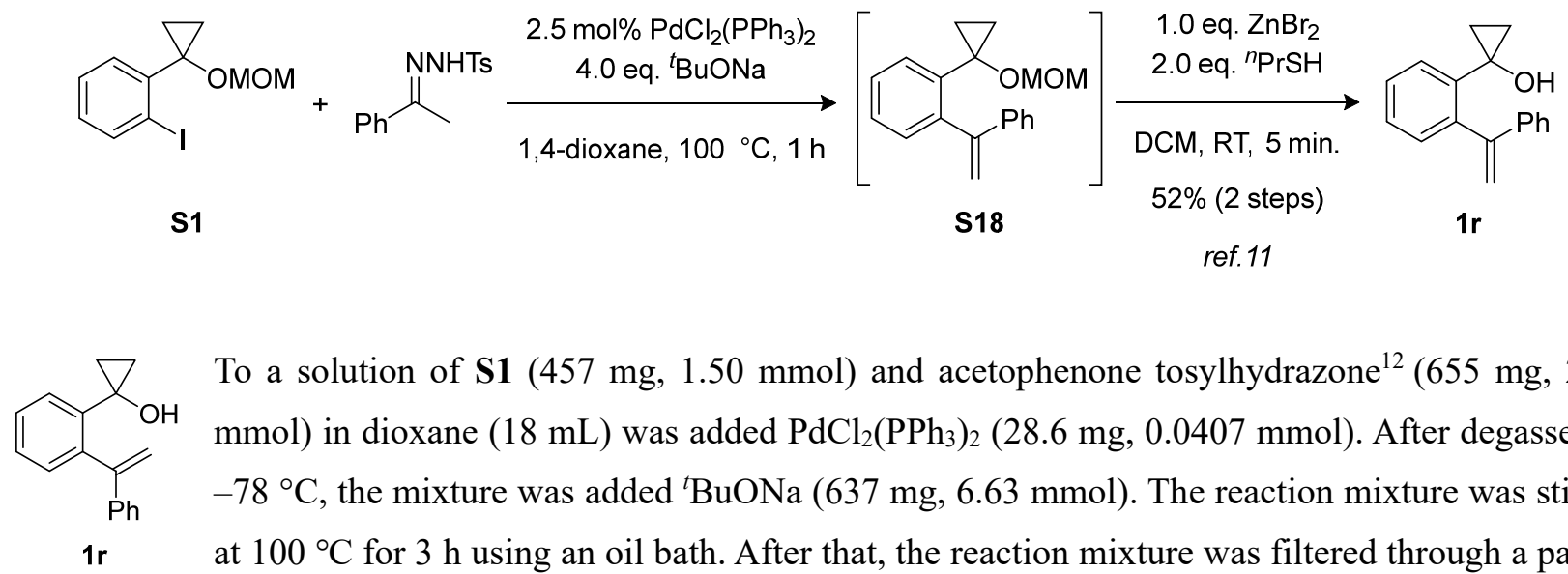

To a solution of $\mathbf{S 1}(457 \mathrm{mg}, 1.50 \mathrm{mmol})$ and acetophenone tosylhydrazone ${ }^{12}(655 \mathrm{mg}, 2.27$ $\mathrm{mmol})$ in dioxane $(18 \mathrm{~mL})$ was added $\mathrm{PdCl}_{2}\left(\mathrm{PPh}_{3}\right)_{2}(28.6 \mathrm{mg}, 0.0407 \mathrm{mmol})$. After degassed at $-78{ }^{\circ} \mathrm{C}$, the mixture was added ${ }^{t} \mathrm{BuONa}(637 \mathrm{mg}, 6.63 \mathrm{mmol})$. The reaction mixture was stirred at $100{ }^{\circ} \mathrm{C}$ for $3 \mathrm{~h}$ using an oil bath. After that, the reaction mixture was filtered through a pad of celite with EtOAc. The filtrate concentrated in vacuo. The obtained crude product was purified by silica gel column chromatography (Hexane/EtOAc $=50: 1)$ to give S18, which still contained a small amount of inseparable impurities. The crude $\mathbf{S 1 8}$ was used for the next reaction without further purification at this stage. To a $10 \mathrm{~mL}$ two-necked flask was charged with dried $\mathrm{ZnBr}_{2}(338 \mathrm{mg}, 1.50 \mathrm{mmol})$, followed by crude $\mathbf{S 1 8}$ in dry DCM $(2.0 \mathrm{~mL})$. After the addition of ${ }^{n} \mathrm{PrSH}(272 \mu \mathrm{L}, 3.00 \mathrm{mmol})$, the reaction mixture was stirred at room temperature for $5 \mathrm{~min}$. The resulting mixture was diluted with $\mathrm{DCM}$ and added sat. aq. $\mathrm{NaHCO}_{3}$, then the mixture was filtered through a pad of Celite. The aqueous layer was separated and further extracted with DCM. The combined organic layer was washed with brine, dried over $\mathrm{MgSO}_{4}$, and concentrated in vacuo. The residue was purified by a silica gel column chromatography (Hexane/EtOAc = 20:1) to furnish 1r $(184 \mathrm{mg}, 52 \%, 2$ steps from S1). Analytical data for 1r: orange oil; ${ }^{1} \mathrm{H}-\mathrm{NMR}\left(400 \mathrm{MHz}, \mathrm{CDCl}_{3}\right) \delta$ 7.52-7.49 (m, 1H), 7.38$7.27(\mathrm{~m}, 8 \mathrm{H}), 5.76(\mathrm{~d}, J=1.2 \mathrm{~Hz}, 1 \mathrm{H}), 5.33(\mathrm{~d}, J=1.2 \mathrm{~Hz}, 1 \mathrm{H}), 1.91(\mathrm{~s}, 1 \mathrm{H}), 0.81$ (dd, $J=7.2,5.2 \mathrm{~Hz}, 2 \mathrm{H})$, $0.79(\mathrm{dd}, J=7.2,5.2 \mathrm{~Hz}, 2 \mathrm{H}) ;{ }^{13} \mathrm{C}-\mathrm{NMR}\left(101 \mathrm{MHz}, \mathrm{CDCl}_{3}\right) \delta 150.3,142.3,142.2,139.6,130.8,130.6,128.7$, 128.3, 128.0, 127.2, 115.9, 56.9, 15.3, One Csp $p^{2}$ signal is missing due to overlapping.; IR (neat) $3444 \mathrm{~cm}^{-1}$; HRMS (DART) $m / z$ : $\left[\mathrm{M}+\mathrm{NH}_{4}\right]^{+}$Calcd for $\mathrm{C}_{17} \mathrm{H}_{20} \mathrm{NO} 254.1545$; Found 254.1539.

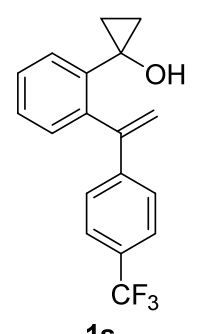

$1 \mathrm{~s}$

This compound was prepared in the same manner as described for $\mathbf{1 r}$ using tosylhydrazone synthesized from 4'-trifluoromethylacetophenone ${ }^{13}$ in $61 \%$ yield for 2 steps. This compound was purified by silica gel column chromatography $($ Hexane/EtOAc $=20: 1)$. Analytical data for 1s: yellow oil; ${ }^{1} \mathrm{H}-\mathrm{NMR}\left(400 \mathrm{MHz}, \mathrm{CDCl}_{3}\right) \delta 7.56(\mathrm{~d}, J=8.4 \mathrm{~Hz}, 2 \mathrm{H}), 7.48(\mathrm{dd}, J=6.4,2.4 \mathrm{~Hz}$, $1 \mathrm{H}), 7.41(\mathrm{~d}, J=8.4 \mathrm{~Hz}, 2 \mathrm{H}), 7.35-7.33(\mathrm{~m}, 2 \mathrm{H}), 7.24(\mathrm{dd}, J=6.4,2.4 \mathrm{~Hz}, 1 \mathrm{H}), 5.87(\mathrm{~s}, 1 \mathrm{H})$, $5.47(\mathrm{~s}, 1 \mathrm{H}), 2.06(\mathrm{~s}, 1 \mathrm{H}), 0.83(\mathrm{dd}, J=7.2,5.2 \mathrm{~Hz}, 2 \mathrm{H}), 0.76(\mathrm{dd}, J=7.2,5.2 \mathrm{~Hz}, 2 \mathrm{H}) ;{ }^{13} \mathrm{C}-$ $\operatorname{NMR}\left(101 \mathrm{MHz}, \mathrm{CDCl}_{3}\right) \delta 148.8,145.3,141.5,139.8,130.9,130.2,130.0$ (q, $\left.J=33 \mathrm{~Hz}\right), 128.5,128.1,127.4$, 125.5 (q, $J=3.8 \mathrm{~Hz}), 118.0,56.9,15.2$, A signal of $C \mathrm{~F}_{3}$ is obscure due to overlapping with aromatic peaks; 
${ }^{19} \mathrm{~F}-\mathrm{NMR}\left(376 \mathrm{MHz}, \mathrm{CDCl}_{3}\right.$ ) $\delta-63.5$; IR (neat) $3420 \mathrm{~cm}^{-1}$; HRMS (DART) $m / z$ : $\left[\mathrm{M}+\mathrm{NH}_{4}\right]^{+}$Calcd for $\mathrm{C}_{18} \mathrm{H}_{19} \mathrm{~F}_{3} \mathrm{NO} 322.1419$; Found 322.1406.

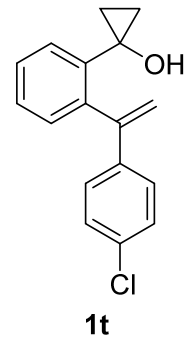

This compound was prepared in the same manner as described for $\mathbf{1 r}$ using tosylhydrazone synthesized from 4'-chloroacetophenone ${ }^{12}$ in $54 \%$ yield for 2 steps. This compound was purified by silica gel column chromatography $($ Hexane $/ E t O A c=20: 1)$. Analytical data for 1t: yellow oil; ${ }^{1} \mathrm{H}-\mathrm{NMR}\left(400 \mathrm{MHz}, \mathrm{CDCl}_{3}\right) \delta 7.49$ (dd, $\left.J=7.2,2.0 \mathrm{~Hz}, 1 \mathrm{H}\right), 7.38-7.33(\mathrm{~m}, 2 \mathrm{H}), 7.32-7.23$ $(\mathrm{m}, 5 \mathrm{H}), 5.78(\mathrm{~d}, J=0.8 \mathrm{~Hz}, 1 \mathrm{H}), 5.36(\mathrm{~d}, J=0.8 \mathrm{~Hz}, 1 \mathrm{H}), 1.81(\mathrm{~s}, 1 \mathrm{H}), 0.84(\mathrm{td}, J=6.4,1.2 \mathrm{~Hz}$, 2H), $0.77(\mathrm{td}, J=6.4,1.2 \mathrm{~Hz}, 2 \mathrm{H}) ;{ }^{13} \mathrm{C}-\mathrm{NMR}\left(101 \mathrm{MHz}, \mathrm{CDCl}_{3}\right) \delta 149.0,141.8,140.4,139.7$, 134.1, 130.8, 130.3, 128.8, 128.4, 128.1, 116.4, 56.9, 15.3, One Csp $p^{2}$ signal is missing due to overlapping.; IR (neat) $3445 \mathrm{~cm}^{-1}$; HRMS (DART) $\mathrm{m} / z$ : $\left[\mathrm{M}+\mathrm{NH}_{4}\right]^{+}$Calcd for $\mathrm{C}_{17} \mathrm{H}_{19} \mathrm{ClNO} 288.1155$; Found 288.1168.

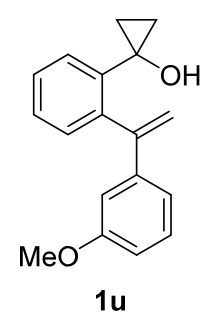

This compound was prepared in the same manner as described for $\mathbf{1 r}$ using tosylhydrazone synthesized from 3'-methoxyacetophenone ${ }^{12}$ in $69 \%$ yield for 2 steps. This compound was purified by silica gel column chromatography $($ Hexane/EtOAc $=10: 1)$. Analytical data for $1 \mathbf{u}$ : colorless oil; ${ }^{1} \mathrm{H}-\mathrm{NMR}\left(400 \mathrm{MHz}, \mathrm{CDCl}_{3}\right) \delta$ 7.51-7.49 (m, 1H), 7.36-7.27 (m, 3H), 7.25-7.21 $(\mathrm{m}, 1 \mathrm{H}), 6.91(\mathrm{t}, J=2.0 \mathrm{~Hz}, 1 \mathrm{H}), 6.86-6.82(\mathrm{~m}, 2 \mathrm{H}), 5.76(\mathrm{~d}, J=1.2 \mathrm{~Hz}, 1 \mathrm{H}), 5.32(\mathrm{~d}, J=1.2$ $\mathrm{Hz}, 1 \mathrm{H}), 3.77$ (s, 3H), 2.03 (s, 1H), 0.86-0.83 (m, 2H), 0.81-0.77 (m, 2H); ${ }^{13} \mathrm{C}-\mathrm{NMR}(101 \mathrm{MHz}$, $\left.\mathrm{CDCl}_{3}\right) \delta 159.9,150.1,143.7,142.3,139.6,130.8,130.6,129.7,128.3,128.0,119.7,116.1,113.4,113.1,56.9$, 55.4, 15.3; IR (neat) $3431 \mathrm{~cm}^{-1}$; HRMS (DART) $\mathrm{m} / z$ : $\left[\mathrm{M}+\mathrm{NH}_{4}\right]^{+}$Calcd for $\mathrm{C}_{18} \mathrm{H}_{22} \mathrm{NO}_{2}$ 284.1651; Found 284.1627.

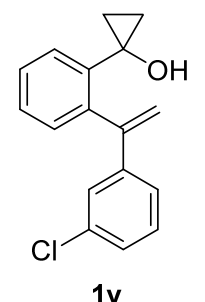

This compound was prepared in the same manner as described for $\mathbf{1 r}$ using tosylhydrazone synthesized from 3'-chlorocetophenone ${ }^{14}$ in $45 \%$ yield for 2 steps. This compound was purified by silica gel column chromatography (Hexane/EtOAc $=20: 1)$. Analytical data for 1v: yellow oil; ${ }^{1} \mathrm{H}-\mathrm{NMR}\left(400 \mathrm{MHz}, \mathrm{CDCl}_{3}\right) \delta$ 7.51-7.48 (m, 1H), 7.39-7.32 (m, 3H), 7.29-7.22 (m, 3H), $7.15(\mathrm{td}, J=6.8,2.0 \mathrm{~Hz}, 1 \mathrm{H}), 5.80(\mathrm{~d}, J=1.2 \mathrm{~Hz}, 1 \mathrm{H}), 5.39(\mathrm{~d}, J=1.2 \mathrm{~Hz}, 1 \mathrm{H}), 2.01(\mathrm{~s}, 1 \mathrm{H})$, $0.87-0.83(\mathrm{~m}, 2 \mathrm{H}), 0.80-0.76(\mathrm{~m}, 2 \mathrm{H}) ;{ }^{13} \mathrm{C}-\mathrm{NMR}\left(101 \mathrm{MHz}, \mathrm{CDCl}_{3}\right) \delta$ 148.9, 143.8, 141.6, 139.7, 134.7, 130.9, 130.3, 129.8, 128.4, 128.2, 128.1, 127.1, 125.3, 117.1, 56.9, 15.3; IR (neat) $3426 \mathrm{~cm}^{-1}$; HRMS (DART) $\mathrm{m} / z$ : $[\mathrm{M}+\mathrm{H}]^{+}$Calcd for $\mathrm{C}_{17} \mathrm{H}_{16} \mathrm{ClO} 271.0876$; Found 271.0890.

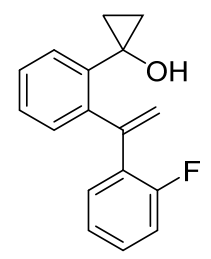

This compound was prepared in the same manner as described for $\mathbf{1 r}$ using tosylhydrazone synthesized from 2'-fluoroacetophenone ${ }^{14}$ in $49 \%$ yield for 2 steps. This compound was purified by silica gel column chromatography (Hexane/EtOAc $=20: 1)$. Analytical data for 1w: colorless oil; ${ }^{1} \mathrm{H}-\mathrm{NMR}\left(400 \mathrm{MHz}, \mathrm{CDCl}_{3}\right) \delta$ 7.48-7.46 (m, 1H), 7.35-7.32 (m, 3H), 7.29-7.23 $1 w$ (m, 1H), 7.13-7.04 (m, 3H), $5.84(\mathrm{~s}, 1 \mathrm{H}), 5.63(\mathrm{~s}, 1 \mathrm{H}), 1.97(\mathrm{~s}, 1 \mathrm{H}), 0.86-0.83(\mathrm{~m}, 2 \mathrm{H}), 0.79-$ $0.76(\mathrm{~m}, 2 \mathrm{H}) ;{ }^{13} \mathrm{C}-\mathrm{NMR}\left(101 \mathrm{MHz}, \mathrm{CDCl}_{3}\right) \delta 160.1(\mathrm{~d}, J=251 \mathrm{~Hz}), 144.0,142.5,139.2,130.7,130.6(\mathrm{~d}, J=$ $2.9 \mathrm{~Hz}), 130.3,129.9(\mathrm{~d}, J=11.5 \mathrm{~Hz}), 129.5(\mathrm{~d}, J=8.7 \mathrm{~Hz}), 128.2(\mathrm{~d}, J=5.8 \mathrm{~Hz}), 124.0(\mathrm{~d}, J=3.8 \mathrm{~Hz}), 120.8$, 
120. $8,116.5(\mathrm{~d}, J=22.2 \mathrm{~Hz}), 56.9,15.1 ;{ }^{19} \mathrm{~F}-\mathrm{NMR}\left(376 \mathrm{MHz}, \mathrm{CDCl}_{3}\right) \delta-115.1$; IR (neat) $3423 \mathrm{~cm}^{-1}$; HRMS (DART) $m / z:[\mathrm{M}+\mathrm{H}]^{+}$Calcd for $\mathrm{C}_{17} \mathrm{H}_{16} \mathrm{FO} 255.1185$; Found 255.1194.

\section{Synthesis and characterization of $1 x$}
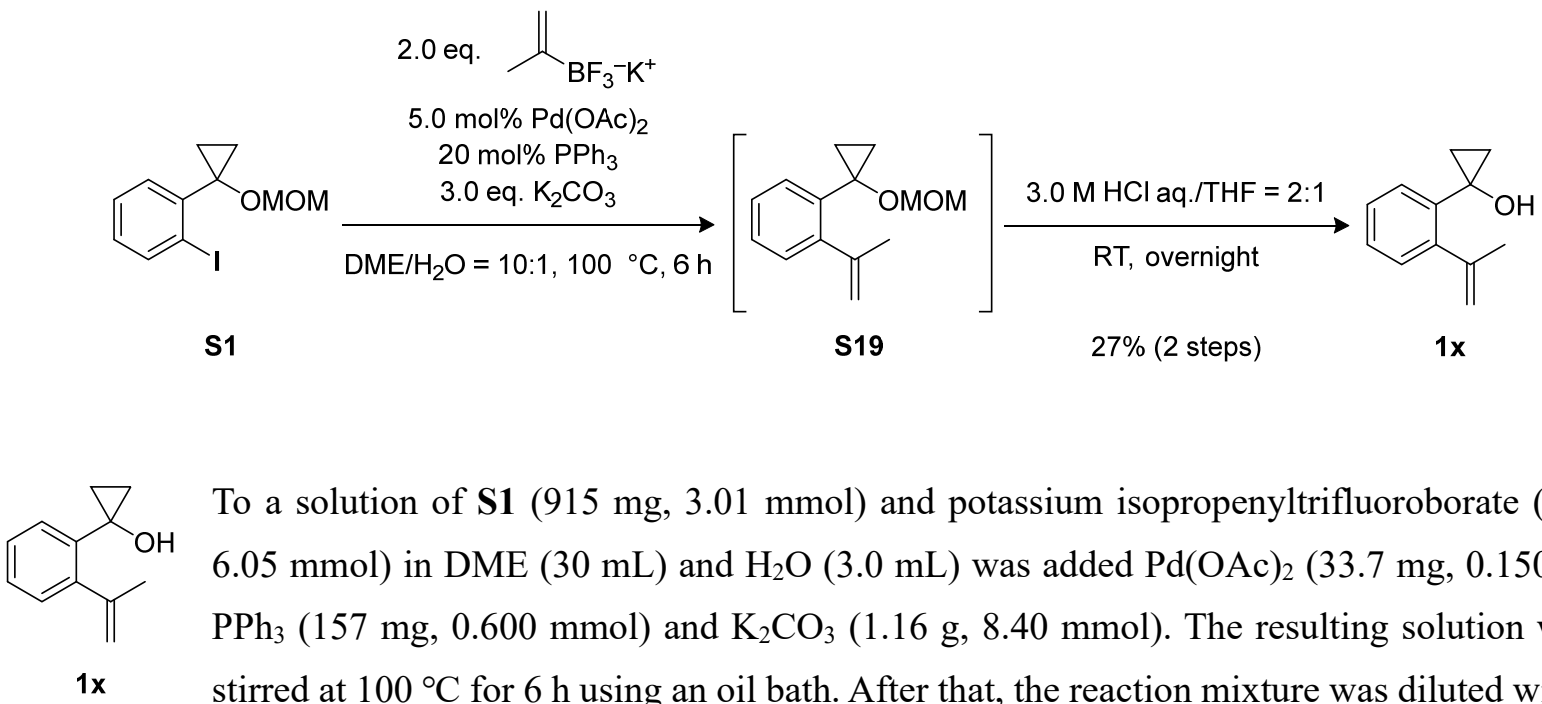

To a solution of $\mathbf{S} 1(915 \mathrm{mg}, 3.01 \mathrm{mmol})$ and potassium isopropenyltrifluoroborate $(895 \mathrm{mg}$, $6.05 \mathrm{mmol})$ in DME $(30 \mathrm{~mL})$ and $\mathrm{H}_{2} \mathrm{O}(3.0 \mathrm{~mL})$ was added $\mathrm{Pd}(\mathrm{OAc})_{2}(33.7 \mathrm{mg}, 0.150 \mathrm{mmol})$, $\mathrm{PPh}_{3}(157 \mathrm{mg}, 0.600 \mathrm{mmol})$ and $\mathrm{K}_{2} \mathrm{CO}_{3}(1.16 \mathrm{~g}, 8.40 \mathrm{mmol})$. The resulting solution was then stirred at $100{ }^{\circ} \mathrm{C}$ for $6 \mathrm{~h}$ using an oil bath. After that, the reaction mixture was diluted with water and extracted with $\mathrm{Et}_{2} \mathrm{O}$. The organic layer was washed with brine, dried over $\mathrm{MgSO}_{4}$, and concentrated in vacuo. The residue was purified by silica gel column chromatography $(\mathrm{Hexane} / \mathrm{EtOAc}=50: 1)$ to furnish $\mathbf{S 1 9}$, which still contained a small amount of inseparable impurities. The crude $\mathbf{S 1 9}$ was used for the next reaction without further purification at this stage. To a THF solution $(3.0 \mathrm{~mL})$ of $\mathbf{S 1 9}$ was added aq. $\mathrm{HCl}(6.0 \mathrm{~mL}, 3 \mathrm{M})$. The resulting mixture was stirred at room temperature overnight. The reaction was quenched with sat. aq. $\mathrm{NaHCO}_{3}$. The aqueous layer was extracted with $\mathrm{Et}_{2} \mathrm{O}$. The combined organic layer was washed with brine, dried over $\mathrm{MgSO}_{4}$, and concentrated in vacuo. The residue was purified by a silica gel column chromatography (Hexane/EtOAc $=20: 1)$ to furnish $\mathbf{1 x}(142 \mathrm{mg}, 27 \%, 2$ steps from S1). Analytical data for 1x: colorless oil; ${ }^{1} \mathrm{H}-\mathrm{NMR}\left(400 \mathrm{MHz}, \mathrm{CDCl}_{3}\right) \delta$ 7.38-7.36 (m, 1H), 7.27-7.21 (m, 2H), 7.17-7.14 (m, 1H), 5.29 (dq, $J=2.8$, $1.6 \mathrm{~Hz}, 1 \mathrm{H}), 5.06(\mathrm{dq}, J=2.8,1.6 \mathrm{~Hz}, 1 \mathrm{H}), 2.74(\mathrm{~s}, 1 \mathrm{H}), 2.20(\mathrm{~s}, 3 \mathrm{H}), 1.11(\mathrm{dd}, J=7.2,5.2 \mathrm{~Hz}, 2 \mathrm{H}), 0.96(\mathrm{dd}$, $J=7.2,5.2 \mathrm{~Hz}, 2 \mathrm{H}) ;{ }^{13} \mathrm{C}-\mathrm{NMR}\left(101 \mathrm{MHz}, \mathrm{CHCl}_{3}\right) \delta 147.2,144.6,138.9,128.8,127.8,127.2,115.6,57.2$, 25.3, 15.1, One Csp signal is missing due to overlapping.; IR (neat) $3420 \mathrm{~cm}^{-1}$; HRMS (DART) $\mathrm{m} / z$ : [M + $\left.\mathrm{NH}_{4}\right]^{+}$Calcd for $\mathrm{C}_{12} \mathrm{H}_{18} \mathrm{NO}$ 192.1388; Found 192.1377. 


\section{Synthesis and characterization of $1 y$}
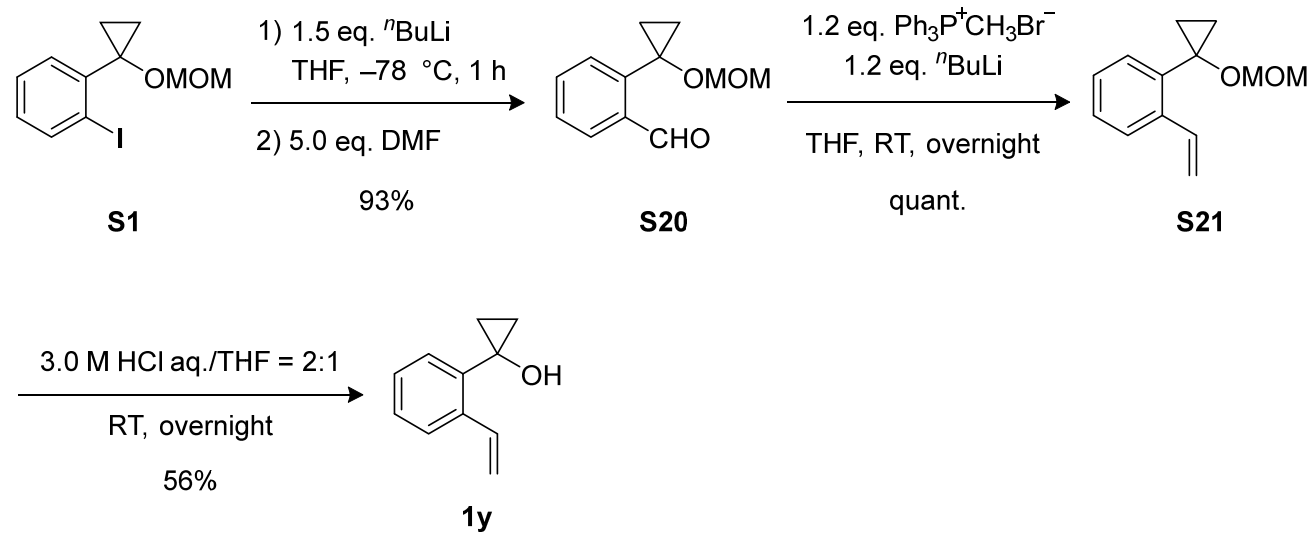

$\mathbf{S 2 0}^{4}$ was prepared according to the previous report.

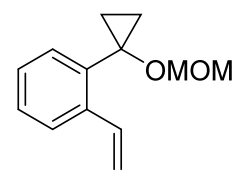

In a $30 \mathrm{~mL}$ two-necked flask, to a suspension of $\mathrm{CH}_{3} \mathrm{PPh}_{3} \mathrm{Br}(429 \mathrm{mg}, 1.20 \mathrm{mmol})$ in dry THF $(5.0 \mathrm{~mL})$ was added a solution of ${ }^{n} \mathrm{BuLi}\left(0.770 \mathrm{~mL}, 1.60 \mathrm{M}\right.$ in hexane) at $0{ }^{\circ} \mathrm{C}$ and the reaction mixture was stirred at room temperature for $0.5 \mathrm{~h}$. To the resultant yellow solution was added S21 a THF solution $(2.0 \mathrm{~mL})$ of $\mathbf{S 2 0}(206 \mathrm{mg}, 1.00 \mathrm{mmol})$ at $0{ }^{\circ} \mathrm{C}$ and the mixture was stirred at room temperature overnight. The reaction was quenched with water. The aqueous layer was extracted with $\mathrm{Et}_{2} \mathrm{O}$. The combined organic layer was washed with brine, dried over $\mathrm{MgSO}_{4}$, and concentrated in vacuo. The residue was purified by a silica gel column chromatography (Hexane/EtOAc $=20: 1)$ to furnish $\mathbf{S 2 1}(205 \mathrm{mg}$, quant.). Analytical data for S21: pale-yellow liquid; ${ }^{1} \mathrm{H}-\mathrm{NMR}\left(400 \mathrm{MHz}, \mathrm{CDCl}_{3}\right) \delta 7.61$ (dd, $J=7.6,1.2 \mathrm{~Hz}$, $1 \mathrm{H}), 7.50(\mathrm{dd}, J=17.6,11.2 \mathrm{~Hz}, 1 \mathrm{H}), 7.34(\mathrm{dd}, J=7.6,1.2 \mathrm{~Hz}, 1 \mathrm{H}), 7.29(\mathrm{td}, J=7.6,1.2 \mathrm{~Hz}, 1 \mathrm{H}), 7.21(\mathrm{td}, J$ $=7.6,1.2 \mathrm{~Hz}, 1 \mathrm{H}), 5.73(\mathrm{dd}, J=17.6,1.2 \mathrm{~Hz}, 1 \mathrm{H}), 5.33(\mathrm{dd}, J=11.2,1.2 \mathrm{~Hz}, 1 \mathrm{H}), 4.56(\mathrm{~s}, 2 \mathrm{H}), 3.16(\mathrm{~s}, 3 \mathrm{H})$, $1.23(\mathrm{dd}, J=7.2,5.2 \mathrm{~Hz}, 2 \mathrm{H}), 0.93(\mathrm{dd}, J=7.2,5.2 \mathrm{~Hz}, 2 \mathrm{H}) ;{ }^{13} \mathrm{C}-\mathrm{NMR}\left(101 \mathrm{MHz}, \mathrm{CDCl}_{3}\right) \delta 138.6,137.0$, 135.0, 130.1, 128.4, 127.3, 125.3, 114.7, 94.9, 60.7, 55.7, 12.6; IR (neat) $1034 \mathrm{~cm}^{-1}$; HRMS (DART) $\mathrm{m} / z$ : [M $\left.+\mathrm{NH}_{4}\right]^{+}$Calcd for $\mathrm{C}_{13} \mathrm{H}_{20} \mathrm{NO}_{2}$ 222.1494; Found 222.1466.

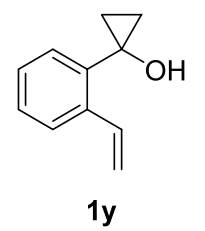

To a THF solution $(2.0 \mathrm{~mL})$ of $\mathbf{S 2 1}(205 \mathrm{mg}, 1.00 \mathrm{mmol})$ was added aq. $\mathrm{HCl}(4.0 \mathrm{~mL}, 3 \mathrm{M})$. The resulting mixture was stirred at room temperature overnight. The reaction was quenched with sat. aq. $\mathrm{NaHCO}_{3}$. The aqueous layer was extracted with $\mathrm{Et}_{2} \mathrm{O}$. The combined organic layer was washed with brine, dried over $\mathrm{MgSO}_{4}$, and concentrated in vacuo. The residue was purified by a silica gel column chromatography (Hexane/EtOAc $=20: 1)$ to furnish $1 \mathbf{y}(89.7 \mathrm{mg}, 56 \%)$. Analytical data for 1y: colorless liquid; ${ }^{1} \mathrm{H}-\mathrm{NMR}\left(400 \mathrm{MHz}, \mathrm{CDCl}_{3}\right) \delta 7.59$ (dd, $\left.J=7.6,1.2 \mathrm{~Hz}, 1 \mathrm{H}\right), 7.48(\mathrm{dd}, J=17.6,11.2 \mathrm{~Hz}$, 1H), 7.37 (dd, $J=7.6,1.2 \mathrm{~Hz}, 1 \mathrm{H}), 7.30$ (td, $J=7.6,1.2 \mathrm{~Hz}, 1 \mathrm{H}), 7.23$ (dd, $J=7.6,1.2 \mathrm{~Hz}, 1 \mathrm{H}), 5.76(\mathrm{dd}, J=$ 17.6, $1.2 \mathrm{~Hz}, 1 \mathrm{H}), 5.38(\mathrm{dd}, J=11.2,1.2 \mathrm{~Hz}, 1 \mathrm{H}), 2.22(\mathrm{~s}, 1 \mathrm{H}), 1.19(\mathrm{dd}, J=7.2,5.2 \mathrm{~Hz}, 2 \mathrm{H}), 0.95(\mathrm{dd}, J=$ 7.2, $5.2 \mathrm{~Hz}, 2 \mathrm{H}) ;{ }^{13} \mathrm{C}-\mathrm{NMR}\left(101 \mathrm{MHz}, \mathrm{CDCl}_{3}\right) \delta 139.1,138.2,134.6,128.8,128.4,127.8,125.8,115.7,56.8$, 14.0; IR (KBr) $3431 \mathrm{~cm}^{-1}$; HRMS (DART) $\mathrm{m} / z$ : $[\mathrm{M}+\mathrm{H}]^{+}$Calcd for $\mathrm{C}_{11} \mathrm{H}_{13} \mathrm{O}$ 161.0966; Found 161.0953. 


\section{Synthesis and characterization of $1 \mathrm{z}$ and $1 \mathrm{aa}$}
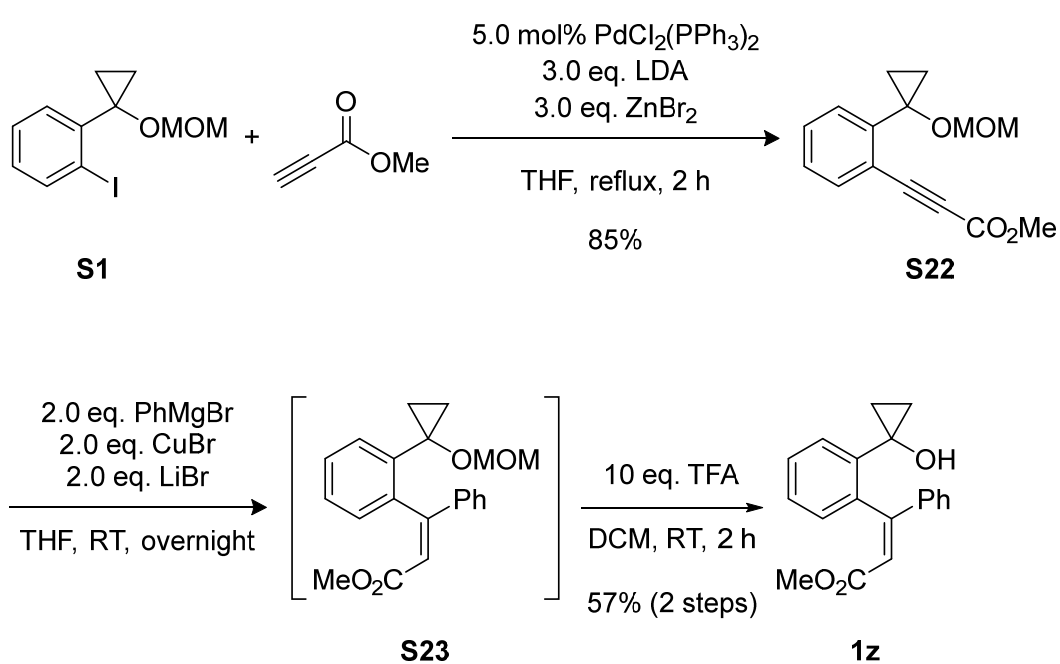

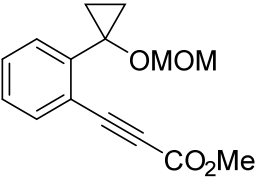

S22

To a solution of ${ }^{i} \operatorname{Pr}_{2} \mathrm{NH}(1.30 \mathrm{~mL}, 7.80 \mathrm{mmol})$ in THF $(15 \mathrm{~mL})$ was added ${ }^{n} \mathrm{BuLi}(5.00 \mathrm{~mL}$, 1.6 $\mathrm{M}$ in hexane) at $0{ }^{\circ} \mathrm{C}$ in a flame-dried flask under Ar atmosphere. After $10 \mathrm{~min}$, the reaction mixture was treated with methyl propiolate $(650 \mu \mathrm{L}, 7.80 \mathrm{mmol})$ at $-78{ }^{\circ} \mathrm{C}$. After additional $10 \mathrm{~min}$, dried $\mathrm{ZnBr}_{2}(1.76 \mathrm{~g}, 7.82 \mathrm{mmol})$ in THF $(6.0 \mathrm{~mL})$ was added and the mixture was stirred for $10 \mathrm{~min}$. S1 (794 mg, $2.60 \mathrm{mmol})$ and $\mathrm{PdCl}_{2}\left(\mathrm{PPh}_{3}\right)_{2}(91.2 \mathrm{mg}, 0.130 \mathrm{mmol})$ was added to the resultant solution. The temperature was raised to room temperature and the mixture was refluxed for 2 $\mathrm{h}$ using an oil bath. The reaction mixture was diluted with $\mathrm{Et}_{2} \mathrm{O}$, washed with sat. aq. $\mathrm{NH}_{4} \mathrm{Cl}$ and then with sat. aq. $\mathrm{NaHCO}_{3}$, dried over $\mathrm{MgSO}_{4}$, filtered, and concentrated in vacuo. The residue was purified by a silica gel column chromatography $($ Hexane/EtOAc $=10: 1)$ to furnish S22 (572 mg, 85\%). Analytical data for S22: dark-yellow oil; ${ }^{1} \mathrm{H}-\mathrm{NMR}\left(400 \mathrm{MHz}, \mathrm{CDCl}_{3}\right) \delta 7.60(\mathrm{dd}, J=7.6,1.2 \mathrm{~Hz}, 1 \mathrm{H}), 7.48(\mathrm{dd}, J=7.6,1.2 \mathrm{~Hz}, 1 \mathrm{H})$, $7.41(\mathrm{td}, J=7.6,1.2 \mathrm{~Hz}, 1 \mathrm{H}), 7.30(\mathrm{td}, J=7.6,1.2 \mathrm{~Hz}, 1 \mathrm{H}), 4.66(\mathrm{~s}, 2 \mathrm{H}), 3.85$ (s, 3H), $3.14(\mathrm{~s}, 3 \mathrm{H}), 1.33(\mathrm{dd}$, $J=7.2,5.6 \mathrm{~Hz}, 2 \mathrm{H}), 1.02(\mathrm{dd}, J=7.2,5.6 \mathrm{~Hz}, 2 \mathrm{H}) ;{ }^{13} \mathrm{C}-\mathrm{NMR}\left(101 \mathrm{MHz}, \mathrm{CDCl}_{3}\right) \delta 154.7,144.0,134.2,130.6$, 130.4, 128.0, 121.2, 95.4, 85.2, 84.6, 61.4, 55.6, 52.9, 13.0; IR (neat) 1712, $1031 \mathrm{~cm}^{-1}$; HRMS (DART) $m / z$ : $\left[\mathrm{M}+\mathrm{NH}_{4}\right]^{+}$Calcd for $\mathrm{C}_{15} \mathrm{H}_{20} \mathrm{NO}_{4} 278.1392$; Found 278.1409 .

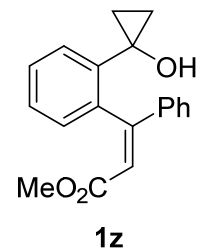

To a solution of dried $\mathrm{CuBr}(430 \mathrm{mg}, 3.0 \mathrm{mmol})$ and dried $\mathrm{LiBr}(259 \mathrm{mg}, 2.98 \mathrm{mmol})$ in THF $(5.0 \mathrm{~mL})$ was added $\mathrm{PhMgBr}\left(2.90 \mathrm{~mL}, 1.00 \mathrm{M}\right.$ in THF) at $0{ }^{\circ} \mathrm{C}$ and the reaction mixture was stirred at $0{ }^{\circ} \mathrm{C}$ for $0.5 \mathrm{~h}$. To the resultant solution was added a THF solution $(1.0 \mathrm{~mL})$ of $\mathbf{S 2 2}$ (258 $\mathrm{mg}, 0.990 \mathrm{mmol}$ ) at $0{ }^{\circ} \mathrm{C}$ and the mixture was stirred at room temperature for $1.0 \mathrm{~h}$. The reaction was quenched with sat. aq. $\mathrm{NH}_{4} \mathrm{Cl}$. The aqueous layer was extracted with $\mathrm{Et}_{2} \mathrm{O}$. The combined organic layer was washed with brine, dried over $\mathrm{MgSO}_{4}$, and concentrated in vacuo. The residue was purified by silica gel column chromatography (Hexane/EtOAc $=20: 1)$ to furnish $\mathbf{S 2 3}$, which still contained a small amount of inseparable impurities. The crude $\mathbf{S 2 3}$ was used for the next reaction without further purification at this stage. To a $50 \mathrm{~mL}$ flask was added $\mathbf{S 2 3}$ followed by degassed DCM (10 mL). TFA (765 $\mu \mathrm{L}$, $10.0 \mathrm{mmol}$ ) was slowly added to the solution. After being stirred at $\mathrm{rt}$ for $2 \mathrm{~h}$ under Ar atmosphere, the reaction 
mixture was diluted with water and extracted with EtOAc. The organic layer was washed with sat. aq. $\mathrm{NaHCO}_{3}$ and brine, dried over $\mathrm{MgSO}_{4}$, and concentrated in vacuo. The residue was purified by silica gel column chromatography (Hexane/EtOAc $=10: 1)$ to furnish $\mathbf{1 z}(94.2 \mathrm{mg}, 32 \%, 2$ steps from S22). Analytical data for 1z: yellow oil; ${ }^{1} \mathrm{H}-\mathrm{NMR}\left(400 \mathrm{MHz}, \mathrm{CDCl}_{3}\right) \delta 7.53(\mathrm{dd}, J=7.6,1.6 \mathrm{~Hz}, 1 \mathrm{H}), 7.41-7.30(\mathrm{~m}, 8 \mathrm{H}), 6.14(\mathrm{~s}, 1 \mathrm{H})$, $3.68(\mathrm{~s}, 3 \mathrm{H}), 1.21(\mathrm{~s}, 1 \mathrm{H}), 0.91-0.88(\mathrm{~m}, 2 \mathrm{H}), 0.86-0.82(\mathrm{~m}, 2 \mathrm{H}) ;{ }^{13} \mathrm{C}-\mathrm{NMR}\left(101 \mathrm{MHz}, \mathrm{CDCl}_{3}\right) \delta 166.6,157.3$, 142.8, 140.0, 139.3, 131.7, 130.5, 129.9, 129.44, 129.36, 128.1, 128.0, 119.6, 56.8, 51.5, 15.7; IR (neat) 3448, $1723 \mathrm{~cm}^{-1}$; HRMS (DART) $\mathrm{m} / z$ : $[\mathrm{M}+\mathrm{H}]^{+}$Calcd for $\mathrm{C}_{19} \mathrm{H}_{19} \mathrm{O}_{3} 295.1334$; Found 295.1323.

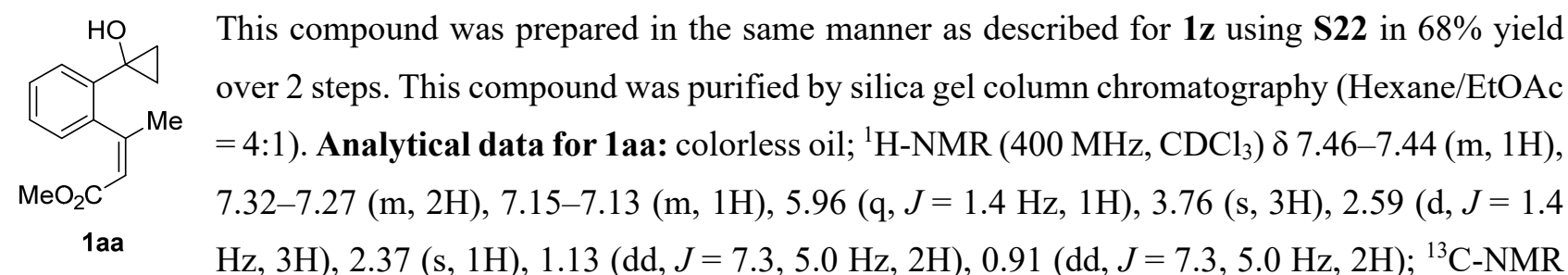
$\left(101 \mathrm{MHz}, \mathrm{CDCl}_{3}\right) \delta 167.0,158.6,144.6,138.5,129.7,128.1,128.0,128.0,119.3,56.9,51.1,21.5,15.1$; IR (neat) $3450,1717 \mathrm{~cm}^{-1}$; HRMS (DART) $\mathrm{m} / z$ : $\left[\mathrm{M}+\mathrm{NH}_{4}\right]^{+}$Calcd for $\mathrm{C}_{14} \mathrm{H}_{20} \mathrm{NO}_{3} 250.1443$; Found 250.1429 .

\section{Synthesis and characterization of $1 \mathrm{bb}$}
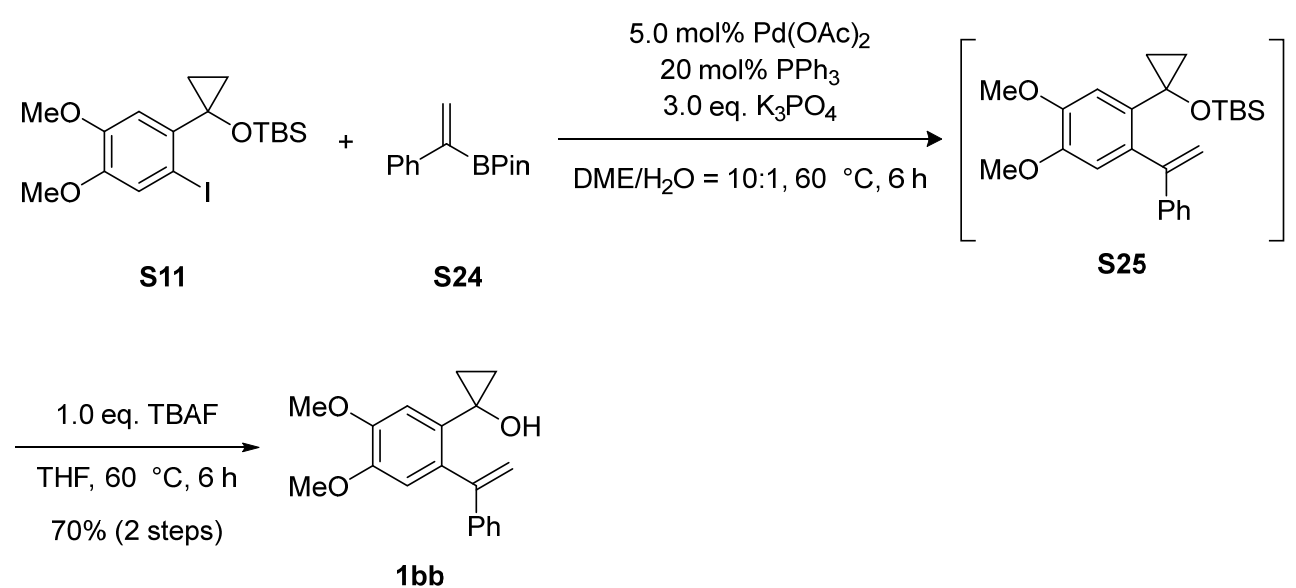

To a solution of $\mathbf{S 1 1}(335 \mathrm{mg}, 1.10 \mathrm{mmol})$ and $\mathbf{S 2 4}{ }^{15}(460 \mathrm{mg}, 2.00 \mathrm{mmol})$ in DME (6.0
$\mathrm{mL})$ and $\mathrm{H}_{2} \mathrm{O}(0.6 \mathrm{~mL})$ was added $\mathrm{Pd}(\mathrm{OAc})_{2}(12.3 \mathrm{mg}, 0.0550 \mathrm{mmol}), \mathrm{PPh}_{3}(57.7 \mathrm{mg}, 0.220$
$\begin{aligned} & \mathrm{mmol}) \text { and } \mathrm{K}_{3} \mathrm{PO}_{4}(636 \mathrm{mg}, 3.00 \mathrm{mmol}) \text {. Then the resulting solution was stirred at } 60{ }^{\circ} \mathrm{C} \\ & \text { for } 6 \mathrm{~h} \text { using an oil bath. After that, the reaction mixture was diluted with water and }\end{aligned}$ extracted with $\mathrm{Et}_{2} \mathrm{O}$. The organic layer was washed with brine, dried over $\mathrm{MgSO}_{4}$, and concentrated in vacuo. The residue was purified by silica gel column chromatography (Hexane/EtOAc $=20: 1)$ to furnish $\mathbf{S 2 5}$, which still contained inseparable impurities. The crude $\mathbf{S 2 5}$ was used for the next reaction without further purification. To a dry THF solution $(6.0 \mathrm{~mL})$ of the crude $\mathbf{S 2 5}$ was added a solution of TBAF (1.0 mL, 1.0 M in THF). The resulting mixture was stirred at $60{ }^{\circ} \mathrm{C}$ for $4 \mathrm{~h}$ using an oil bath. The reaction was quenched with sat. aq. $\mathrm{NaHCO}_{3}$. The aqueous layer was extracted with $\mathrm{Et}_{2} \mathrm{O}$. The combined organic layer was washed with brine, dried over 
$\mathrm{MgSO}_{4}$, and concentrated in vacuo. The residue was purified by a silica gel column chromatography (Hexane/EtOAc $=5: 1)$ to furnish $\mathbf{1 b b}(207 \mathrm{mg}, 70 \%, 2$ steps from S11). Analytical data for 1bb: colorless oil; ${ }^{1} \mathrm{H}-\mathrm{NMR}\left(400 \mathrm{MHz}, \mathrm{CDCl}_{3}\right) \delta$ 7.35-7.28 (m, 5H), $7.05(\mathrm{~s}, 1 \mathrm{H}), 6.81(\mathrm{~s}, 1 \mathrm{H}), 5.72(\mathrm{~d}, J=1.2 \mathrm{~Hz}, 1 \mathrm{H}), 5.31$ $(\mathrm{d}, J=1.2 \mathrm{~Hz}, 1 \mathrm{H}), 3.93(\mathrm{~s}, 3 \mathrm{H}), 3.91(\mathrm{~s}, 3 \mathrm{H}), 1.76(\mathrm{~s}, 1 \mathrm{H}), 0.80(\mathrm{br}, \mathrm{s}, 4 \mathrm{H}) ;{ }^{13} \mathrm{C}-\mathrm{NMR}\left(101 \mathrm{MHz}, \mathrm{CHCl}_{3}\right) \delta$ 150.1, 148.5, 148.3, 142.6, 134.9, 132.1, 128.7, 128.3, 127.2, 115.5, 113.9, 113.7, 56.8, 56.1, 56.0, 15.7; IR (neat) $3544 \mathrm{~cm}^{-1}$; HRMS (DART) $\mathrm{m} / z$ : $[\mathrm{M}+\mathrm{H}]^{+}$Calcd for $\mathrm{C}_{19} \mathrm{H}_{21} \mathrm{O}_{3}$ 297.1491; Found 297.1486. 


\section{Representative Procedure for the Ring Expansion of 1 (Table 1, entry 10)}<smiles>CCOC(=O)/C=C/c1ccccc1C1(O)CC1</smiles>

$1 \mathrm{a}$

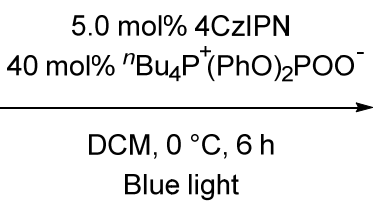

$85 \%$<smiles>CCOC(=O)CC1CCC(=O)c2ccccc21</smiles>

2a

4CzIPN (3.94 mg, $0.00500 \mathrm{mmol}),{ }^{n} \mathrm{Bu}_{4} \mathrm{P}^{+}(\mathrm{PhO})_{2} \mathrm{POO}^{-}(20.3 \mathrm{mg}, 0.0400 \mathrm{mmol})$, and 1a (23.2 mg, $\left.0.100 \mathrm{mmol}\right)$ were dissolved in degassed dry DCM $(1.0 \mathrm{~mL})$ under Ar atmosphere. A LED lamp was put into the reaction mixture in the Schlenk tube, and the reaction mixture was stirred at $0{ }^{\circ} \mathrm{C}$ under irradiation. (Figure S1). After stirring at $0{ }^{\circ} \mathrm{C}$ for $6 \mathrm{~h}$, the resulting solution was concentrated. The residue was then purified by a silica gel column chromatography $($ Hexane/EtOAc $=10: 1)$ to give $\mathbf{2 a}(19.7 \mathrm{mg}, 85 \%)$ as a pale-yellow oil.

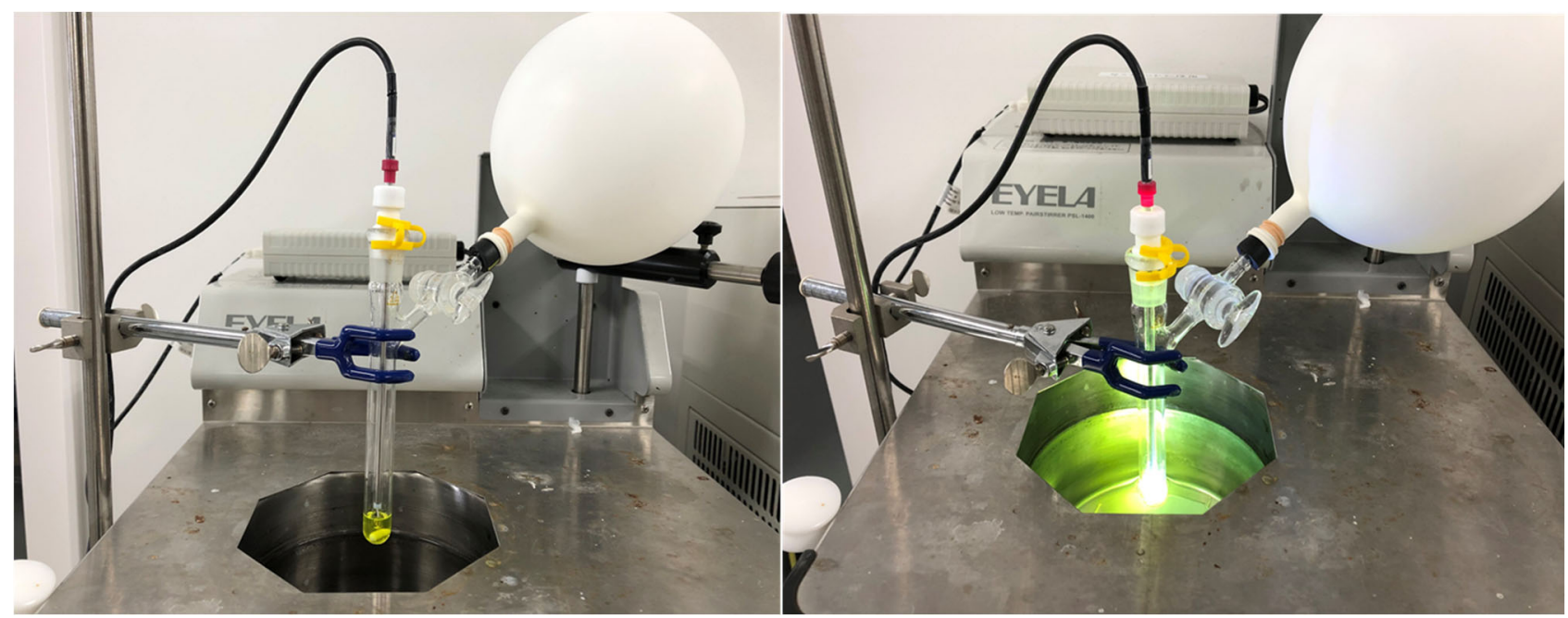

Figure S1. Reaction setup.

\section{Characterization of $4 a$}<smiles>CCOC(=O)/C=C\c1ccccc1C1(O)CC1</smiles>

This compound was purified by silica gel column chromatography $($ Hexane/EtOAc $=10: 1)$ and obtained in $31 \%$ yield $\left(7.2 \mathrm{mg}\right.$ ) as a yellow oil (Table 1, entry 2). Analytical data for $4 \mathrm{a}:{ }^{1} \mathrm{H}$ $\operatorname{NMR}\left(400 \mathrm{MHz}, \mathrm{CDCl}_{3}\right) \delta 7.52(\mathrm{~d}, J=11.6 \mathrm{~Hz}, 1 \mathrm{H}), 7.44(\mathrm{dd}, J=7.6,1.6 \mathrm{~Hz}, 1 \mathrm{H}), 7.32-7.24$ $(\mathrm{m}, 3 \mathrm{H}), 6.11(\mathrm{~d}, J=11.6 \mathrm{~Hz}, 1 \mathrm{H}), 4.08(\mathrm{q}, J=7.2 \mathrm{~Hz}, 2 \mathrm{H}), 2.93(\mathrm{~s}, 1 \mathrm{H}), 1.15$ (t, $J=7.2 \mathrm{~Hz}$, $3 \mathrm{H}), 1.11(\mathrm{dd}, J=7.2,5.2 \mathrm{~Hz}, 2 \mathrm{H}), 0.90(\mathrm{dd}, J=7.2,5.2 \mathrm{~Hz}, 2 \mathrm{H}) ;{ }^{13} \mathrm{C}-\mathrm{NMR}\left(101 \mathrm{MHz}, \mathrm{CDCl}_{3}\right)$ $\delta 166.7,143.5,139.6,136.8,129.0,128.8,128.5,127.7,121.5,60.5,57.2,14.1,14.0$; IR (neat) 3402, 1707 $\mathrm{cm}^{-1}$; HRMS (DART) $\mathrm{m} / z$ : [M $\left.+\mathrm{NH}_{4}\right]^{+}$Calcd for $\mathrm{C}_{14} \mathrm{H}_{20} \mathrm{NO}_{3} 250.1443$; Found 250.1445. 


\section{Characterization of 1-Tetralones 2 and 1-Benzosuberones 3}

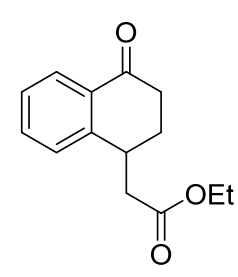

According to the representative procedure, $2 \mathbf{a}$ was obtained in $85 \%$ yield as a pale-yellow oil after purification by silica gel column chromatography $($ Hexane/EtOAc $=10: 1)$. Analytical data for 2a: ${ }^{1} \mathrm{H}-\mathrm{NMR}\left(400 \mathrm{MHz}, \mathrm{CDCl}_{3}\right) \delta 8.04(\mathrm{~d}, J=7.6 \mathrm{~Hz}, 1 \mathrm{H}), 7.51(\mathrm{t}, J=7.6 \mathrm{~Hz}, 1 \mathrm{H})$, $7.35(\mathrm{t}, J=7.6 \mathrm{~Hz}, 1 \mathrm{H}), 7.31(\mathrm{~d}, J=7.6 \mathrm{~Hz}, 1 \mathrm{H}), 4.19(\mathrm{q}, J=7.2 \mathrm{~Hz}, 2 \mathrm{H}), 3.57-3.51(\mathrm{~m}, 1 \mathrm{H})$, 2.82-2.73 (m, 2H), 2.69-2.61 (m, 2H), 2.35-2.27 (m, 1H), 2.11-2.04 (m, 1H), 1.27 (t, J= 7.2 $\mathrm{Hz}, 3 \mathrm{H}) ;{ }^{13} \mathrm{C}-\mathrm{NMR}\left(101 \mathrm{MHz}, \mathrm{CDCl}_{3}\right) \delta 197.7,172.1,146.3,133.9,132.1,127.8,127.6,127.3$, 60.8, 39.8, 35.2, 34.9, 27.5, 14.3; IR (neat) 1732, $1685 \mathrm{~cm}^{-1}$; HRMS (DART) $\mathrm{m} / z$ : $\left[\mathrm{M}+\mathrm{NH}_{4}\right]^{+}$Calcd for $\mathrm{C}_{14} \mathrm{H}_{20} \mathrm{NO}_{3} 250.1443$; Found 250.1434.

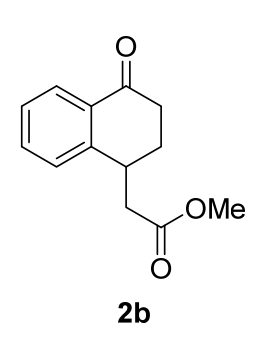

According to the representative procedure, $\mathbf{2} \mathbf{b}$ was obtained in $78 \%$ yield as a pale-yellow oil after purification by silica gel column chromatography $($ Hexane/EtOAc $=10: 1)$. Analytical data for $2 \mathbf{b}:{ }^{1} \mathrm{H}-\mathrm{NMR}\left(400 \mathrm{MHz}, \mathrm{CDCl}_{3}\right) \delta 8.05(\mathrm{dd}, J=7.6,1.2 \mathrm{~Hz}, 1 \mathrm{H}), 7.51(\operatorname{td}, J=7.6$, $1.2 \mathrm{~Hz}, 1 \mathrm{H}), 7.35$ (td, $J=7.6,1.2 \mathrm{~Hz}, 1 \mathrm{H}), 7.30$ (d, $J=7.6 \mathrm{~Hz}, 1 \mathrm{H}), 3.73(\mathrm{~s}, 3 \mathrm{H}), 3.57-3.51$ $(\mathrm{m}, 1 \mathrm{H}), 2.82-2.73(\mathrm{~m}, 2 \mathrm{H}), 2.71-2.61(\mathrm{~m}, 2 \mathrm{H}), 2.35-2.27(\mathrm{~m}, 1 \mathrm{H}), 2.11-2.03(\mathrm{~m}, 1 \mathrm{H}) ;{ }^{13} \mathrm{C}-$ $\operatorname{NMR}\left(101 \mathrm{MHz}, \mathrm{CDCl}_{3}\right) \delta 197.7,172.5,146.2,133.9,132.1,127.8,127.7,127.3,52.0,39.5$, 35.2, 34.8, 27.5; IR (neat) 1736, $1685 \mathrm{~cm}^{-1}$; HRMS (DART) $\mathrm{m} / z$ : $\left[\mathrm{M}+\mathrm{NH}_{4}\right]^{+}$Calcd for $\mathrm{C}_{13} \mathrm{H}_{18} \mathrm{NO}_{3} 236.1287$; Found 236.1261.

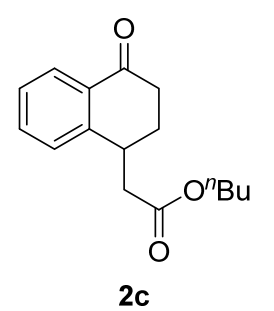

According to the representative procedure, $2 \mathbf{c}$ was obtained in $87 \%$ yield as a pale-yellow oil after purification by silica gel column chromatography (Hexane/EtOAc $=10: 1)$. Analytical data for $2 \mathrm{c}:{ }^{1} \mathrm{H}-\mathrm{NMR}\left(400 \mathrm{MHz}, \mathrm{CDCl}_{3}\right) \delta 8.04(\mathrm{dd}, J=7.6,1.6 \mathrm{~Hz}, 1 \mathrm{H}), 7.51$ $(\mathrm{td}, J=7.6,1.6 \mathrm{~Hz}, 1 \mathrm{H}), 7.35(\mathrm{t}, J=7.6 \mathrm{~Hz}, 1 \mathrm{H}), 7.31(\mathrm{~d}, J=7.6 \mathrm{~Hz}, 1 \mathrm{H}), 4.13(\mathrm{t}, J=6.4$ $\mathrm{Hz}, 2 \mathrm{H}), 3.57-3.51(\mathrm{~m}, 1 \mathrm{H}), 2.82-2.74(\mathrm{~m}, 2 \mathrm{H}), 2.70-2.61(\mathrm{~m}, 2 \mathrm{H}), 2.35-2.27(\mathrm{~m}, 1 \mathrm{H})$, $2.11-2.04(\mathrm{~m}, 1 \mathrm{H}), 1.66-1.58(\mathrm{~m}, 2 \mathrm{H}), 1.41-1.32(\mathrm{~m}, 2 \mathrm{H}), 0.93(\mathrm{t}, J=7.2 \mathrm{~Hz}, 3 \mathrm{H}) ;{ }^{13} \mathrm{C}-$ $\operatorname{NMR}\left(101 \mathrm{MHz}, \mathrm{CDCl}_{3}\right) \delta$ 197.7, 172.2, 146.3, 133.9, 132.1, 127.8, 127.6, 127.3, 64.8, 39.8, 35.2, 34.9, 30.7, 27.5, 19.2, 13.8; IR (neat) 1732, $1686 \mathrm{~cm}^{-1}$; HRMS (DART) $\mathrm{m} / \mathrm{z}$ : $\left[\mathrm{M}+\mathrm{NH}_{4}\right]^{+}$Calcd for $\mathrm{C}_{16} \mathrm{H}_{24} \mathrm{NO}_{3} 278.1756$; Found 278.1760.

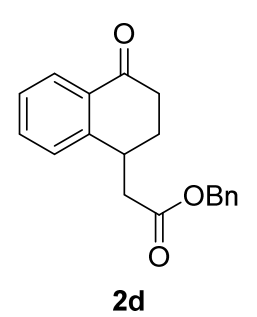

According to the representative procedure, $\mathbf{2 d}$ was obtained in $60 \%$ yield as a pale-yellow oil after purification by silica gel column chromatography $($ Hexane/EtOAc $=10: 1)$. Analytical data for 2d: ${ }^{1} \mathrm{H}-\mathrm{NMR}\left(400 \mathrm{MHz}, \mathrm{CDCl}_{3}\right) \delta 8.04(\mathrm{~d}, J=7.6 \mathrm{~Hz}, 1 \mathrm{H}), 7.46(\mathrm{td}, J=7.6,1.6$ $\mathrm{Hz}, 1 \mathrm{H}), 7.40-7.31(\mathrm{~m}, 6 \mathrm{H}), 7.26(\mathrm{~d}, J=7.6 \mathrm{~Hz}, 1 \mathrm{H}), 5.17(\mathrm{~s}, 2 \mathrm{H}), 3.58-3.52(\mathrm{~m}, 1 \mathrm{H}), 2.84$ $2.58(\mathrm{~m}, 4 \mathrm{H}), 2.33-2.24(\mathrm{~m}, 1 \mathrm{H}), 2.08-2.02(\mathrm{~m}, 1 \mathrm{H}) ;{ }^{13} \mathrm{C}-\mathrm{NMR}\left(101 \mathrm{MHz}, \mathrm{CDCl}_{3}\right) \delta 197.6$, 171.9, 146.1, 135.7, 133.9, 132.1, 128.7, 128.51, 128.45, 127.8, 127.7, 127.3, 66.7, 39.7, 35.2, 
34.9, 27.5; IR (neat) 1733, $1685 \mathrm{~cm}^{-1}$; HRMS (DART) $\mathrm{m} / z$ : $\left[\mathrm{M}+\mathrm{NH}_{4}\right]^{+}$Calcd for $\mathrm{C}_{19} \mathrm{H}_{22} \mathrm{NO}_{3} 312.1600$; Found 312.1590 .

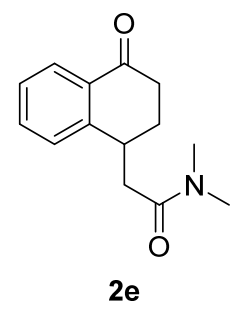

According to the representative procedure, $2 \mathbf{e}$ was obtained in $81 \%$ yield as a yellow amorphas solid after purification by silica gel column chromatography (Hexane/EtOAc $=5: 1$ ). Analytical data for $2 \mathrm{e}:{ }^{1} \mathrm{H}-\mathrm{NMR}\left(400 \mathrm{MHz}, \mathrm{CDCl}_{3}\right) \delta 8.04(\mathrm{dd}, J=7.6,1.2 \mathrm{~Hz}, 1 \mathrm{H}), 7.50$ (td, $J=7.6,1.2 \mathrm{~Hz}, 1 \mathrm{H}), 7.34(\mathrm{t}, J=7.6 \mathrm{~Hz}, 1 \mathrm{H}), 7.32(\mathrm{~d}, J=7.6 \mathrm{~Hz}, 1 \mathrm{H}), 3.70-3.66(\mathrm{~m}, 1 \mathrm{H})$, $2.99(\mathrm{~s}, 3 \mathrm{H}), 2.91(\mathrm{~s}, 3 \mathrm{H}), 2.80-2.62(\mathrm{~m}, 4 \mathrm{H}), 2.38-2.29(\mathrm{~m}, 1 \mathrm{H}), 2.15-2.07(\mathrm{~m}, 1 \mathrm{H}) ;{ }^{13} \mathrm{C}-$ NMR (101 MHz, $\left.\mathrm{CDCl}_{3}\right) \delta 198.1,171.1,147.3,133.9,132.1,128.1,127.5,127.1,38.2,37.4,35.7,35.3,34.7$, 27.6; IR (KBr) 1682, $1623 \mathrm{~cm}^{-1}$; HRMS (DART) $m / z$ : $\left[\mathrm{M}+\mathrm{NH}_{4}\right]^{+}$Calcd for $\mathrm{C}_{14} \mathrm{H}_{21} \mathrm{~N}_{2} \mathrm{O}_{2}$ 249.1603; Found 249.1578 .

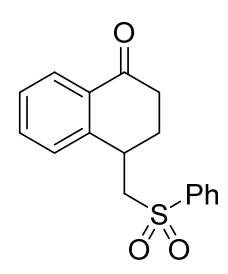

The reaction was performed for 3 h. 2 f was obtained in $68 \%$ yield as a yellow oil after purification by silica gel column chromatography (Hexane/EtOAc $=10: 1)$. Analytical data for 2f: ${ }^{1} \mathrm{H}-\mathrm{NMR}\left(400 \mathrm{MHz}, \mathrm{CDCl}_{3}\right) \delta 8.01(\mathrm{dd}, J=8.0,1.6 \mathrm{~Hz}, 1 \mathrm{H}), 7.99-7.97(\mathrm{~m}, 2 \mathrm{H}), 7.72-$ 7.67 (m, 1H), $7.63-7.59$ (m, 2H), 7.49 (td, $J=7.6,1.6 \mathrm{~Hz}, 1 \mathrm{H}), 7.35$ (td, $J=7.6,0.8 \mathrm{~Hz}, 1 \mathrm{H})$, $7.21(\mathrm{~d}, J=8.0 \mathrm{~Hz}, 1 \mathrm{H}), 3.78-3.73(\mathrm{~m}, 1 \mathrm{H}), 3.48(\mathrm{dd}, J=14.0,9.6 \mathrm{~Hz}, 1 \mathrm{H}), 3.31$ (dd, $J=14.8$, $3.6 \mathrm{~Hz}, 1 \mathrm{H}), 2.80-2.64(\mathrm{~m}, 2 \mathrm{H}), 2.48-2.45(\mathrm{~m}, 1 \mathrm{H}), 2.43-2.35(\mathrm{~m}, 1 \mathrm{H}) ;{ }^{13} \mathrm{C}-\mathrm{NMR}(101 \mathrm{MHz}$, $\left.\mathrm{CDCl}_{3}\right) \delta 197.0,144.5,139.8,134.2,134.1,132.2,129.6,128.2,128.0,127.8,59.7,34.2,33.1,26.3$, One Csp ${ }^{2}$ signal is missing due to overlapping.; IR (neat) $1685,1303 \mathrm{~cm}^{-1}$; HRMS (DART) $m / z$ : $\left[\mathrm{M}+\mathrm{NH}_{4}\right]^{+}$Calcd for $\mathrm{C}_{17} \mathrm{H}_{20} \mathrm{NO}_{3} \mathrm{~S} 318.1164$; Found 318.1159 .

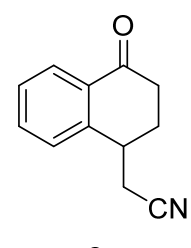

The reaction was performed at $30{ }^{\circ} \mathrm{C} . \mathbf{2 g}$ was obtained in $44 \%$ yield as a pale-yellow oil after purification by silica gel column chromatography (Hexane/EtOAc $=10: 1) .{ }^{1} \mathrm{H}$ NMR analysis of the crude product mixture showed that $\mathbf{1 g}$ was not detected and noticeable side products were not observed. Analytical data for $2 \mathrm{~g}:{ }^{1} \mathrm{H}-\mathrm{NMR}\left(400 \mathrm{MHz}, \mathrm{CDCl}_{3}\right) \delta 8.09(\mathrm{dd}, J=7.6,1.2 \mathrm{~Hz}$, $2 \mathrm{~g}$ $1 \mathrm{H}), 7.59(\mathrm{td}, J=7.6,1.2 \mathrm{~Hz}, 1 \mathrm{H}), 7.42(\mathrm{t}, J=7.6 \mathrm{~Hz}, 1 \mathrm{H}), 7.36(\mathrm{~d}, J=7.6 \mathrm{~Hz}, 1 \mathrm{H}), 3.43-3.37$ (m, 1H), 2.87-2.66 (m, 4H), 2.50-2.41 (m, 1H), 2.29-2.22 (m, 1H); ${ }^{13} \mathrm{C}-\mathrm{NMR}\left(101 \mathrm{MHz}, \mathrm{CDCl}_{3}\right) \delta 196.4$, 143.1, 134.3, 132.1, 128.3, 128.1, 127.5, 118.0, 35.1, 31.2, 27.5, 23.1; IR (neat) 2248, $1685 \mathrm{~cm}^{-1}$; HRMS (DART) $m / z$ : $\left[\mathrm{M}+\mathrm{NH}_{4}\right]^{+}$Calcd for $\mathrm{C}_{12} \mathrm{H}_{15} \mathrm{~N}_{2} \mathrm{O}$ 203.1184; Found 203.1188.

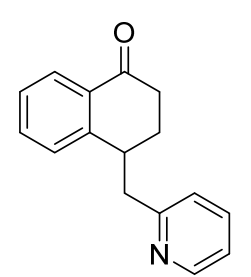

$2 \mathrm{~h}$

The reaction was performed at $30{ }^{\circ} \mathrm{C} . \mathbf{2} \mathbf{h}$ was obtained in $50 \%$ yield as a pale-yellow oil after purification by silica gel column chromatography (Hexane/EtOAc $=10: 1) .{ }^{1} \mathrm{H}$ NMR analysis of the crude product mixture showed that $\mathbf{1 h}$ was not detected and noticeable side products were not observed. Analytical data for $\mathbf{2 h}:{ }^{1} \mathrm{H}-\mathrm{NMR}\left(400 \mathrm{MHz}, \mathrm{CDCl}_{3}\right) \delta 8.62(\mathrm{dd}, J=4.8$, $1.2 \mathrm{~Hz}, 1 \mathrm{H}), 8.06(\mathrm{dd}, J=7.6,1.2 \mathrm{~Hz}, 1 \mathrm{H}), 7.61(\mathrm{td}, J=7.6,2.0 \mathrm{~Hz}, 1 \mathrm{H}), 7.44(\mathrm{td}, J=7.6$, $2.0 \mathrm{~Hz}, 1 \mathrm{H}), 7.33(\mathrm{td}, J=7.6,1.2 \mathrm{~Hz}, 1 \mathrm{H}), 7.25-7.10(\mathrm{~m}, 2 \mathrm{H}), 7.05(\mathrm{~d}, J=7.6 \mathrm{~Hz}, 1 \mathrm{H}), 3.63-$ $3.56(\mathrm{~m}, 1 \mathrm{H}), 3.26(\mathrm{dd}, J=13.6,6.4 \mathrm{~Hz}, 1 \mathrm{H}), 3.07(\mathrm{dd}, J=13.6,9.2 \mathrm{~Hz}, 1 \mathrm{H}), 2.85(\mathrm{ddd}, J=17.2,12.0,5.2$ 
$\mathrm{Hz}, 1 \mathrm{H}), 2.61(\mathrm{dt}, J=17.6,5.2 \mathrm{~Hz}, 1 \mathrm{H}), 2.23-2.14(\mathrm{~m}, 1 \mathrm{H}), 1.99-1.92(\mathrm{~m}, 1 \mathrm{H}) ;{ }^{13} \mathrm{C}-\mathrm{NMR}\left(101 \mathrm{MHz}, \mathrm{CDCl}_{3}\right)$ $\delta$ 198.3, 159.7, 149.7, 147.4, 136.5, 133.6, 132.1, 128.4, 127.5, 127.0, 123.9, 121.7, 43.5, 38.4, 34.9, 26.6; IR (neat) $1683 \mathrm{~cm}^{-1}$; HRMS (DART) $\mathrm{m} / z$ : $\left[\mathrm{M}+\mathrm{NH}_{4}\right]^{+}$Calcd for $\mathrm{C}_{16} \mathrm{H}_{16} \mathrm{NO} 238.1232$; Found 238.1205.

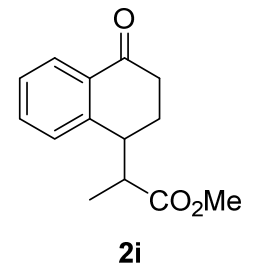

According to the representative procedure, $2 \mathbf{i}$ was obtained in $79 \%$ yield as a yellow oil after purification by silica gel column chromatography (Hexane/EtOAc $=10: 1)$. 2i was analyzed as a mixture of diastereomers (major/minor $=10: 1)$. Analytical data for $2 \mathbf{i}:{ }^{1} \mathrm{H}-\mathrm{NMR}(400$ $\left.\mathrm{MHz}, \mathrm{CDCl}_{3}\right)$ major $\delta 8.04(\mathrm{dd}, J=7.2,1.2 \mathrm{~Hz}, 1 \mathrm{H}), 7.50(\mathrm{td}, J=7.2,1.2 \mathrm{~Hz}, 1 \mathrm{H}), 7.36(\mathrm{td}$, $J=7.2,1.2 \mathrm{~Hz}, 1 \mathrm{H}), 7.25(\mathrm{~d}, J=7.2 \mathrm{~Hz}, 1 \mathrm{H}), 3.74(\mathrm{~s}, 3 \mathrm{H}), 3.15-3.10(\mathrm{~m}, 1 \mathrm{H}), 2.98-2.71(\mathrm{~m}$, 2H), 2.62 (ddd, $J=18.8,5.2,3.6 \mathrm{~Hz}, 1 \mathrm{H}), 2.31-2.17(\mathrm{~m}, 1 \mathrm{H}), 2.08-2.01(\mathrm{~m}, 1 \mathrm{H}), 1.14$ (d, $J=7.2 \mathrm{~Hz}, 3 \mathrm{H})$, minor $\delta 8.04(\mathrm{dd}, J=7.2,1.2 \mathrm{~Hz}, 1 \mathrm{H}), 7.47(\mathrm{td}, J=7.2,1.2 \mathrm{~Hz}, 1 \mathrm{H}), 7.34(\mathrm{td}, J=7.2,1.2 \mathrm{~Hz}, 1 \mathrm{H}), 7.22(\mathrm{~d}, J$ $=7.2 \mathrm{~Hz}, 1 \mathrm{H}), 3.57(\mathrm{~s}, 3 \mathrm{H}), 3.34-3.29(\mathrm{~m}, 1 \mathrm{H}), 2.98-2.71(\mathrm{~m}, 2 \mathrm{H}), 2.62(\mathrm{ddd}, J=18.8,5.2,3.6 \mathrm{~Hz}, 1 \mathrm{H}), 2.31-$ $2.17(\mathrm{~m}, 2 \mathrm{H}), 1.23(\mathrm{~d}, J=7.2 \mathrm{~Hz}, 3 \mathrm{H}) ;{ }^{13} \mathrm{C}-\mathrm{NMR}\left(101 \mathrm{MHz}, \mathrm{CDCl}_{3}\right)$ major $\delta 197.9,176.2,144.6,133.0,132.3$, $129.9,127.8,127.6,52.0,42.8,41.5,34.4,26.5,16.4$, minor $\delta 197.7,176.0,144.6,133.5,132.4,129.9,127.8$, 127.4, 51.7, 42.9, 40.7, 35.1, 24.3, 14.4; IR (neat) 1734, $1685 \mathrm{~cm}^{-1}$; HRMS (DART) $\mathrm{m} / z$ : $\left[\mathrm{M}+\mathrm{NH}_{4}\right]^{+}$Calcd for $\mathrm{C}_{14} \mathrm{H}_{20} \mathrm{NO}_{3} 250.1443$; Found 250.1428.

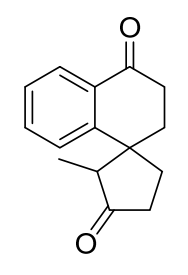

The reaction was performed at $-20{ }^{\circ} \mathrm{C}$. $\mathbf{2} \mathbf{j}$ was obtained in $49 \%$ yield as a colorless oil after purification by silica gel column chromatography $($ Hexane/EtOAc $=5: 1)$ and analyzed as a mixture of diastereomers (major/minor $=1.7: 1)$. Analytical data for $\mathbf{2 j}:{ }^{1} \mathrm{H}-\mathrm{NMR}\left(400 \mathrm{MHz}, \mathrm{CDCl}_{3}\right)$ major $\delta 8.14(\mathrm{dd}, J=7.6,1.2 \mathrm{~Hz}, 1 \mathrm{H}), 7.46(\mathrm{td}, J=7.6,1.6 \mathrm{~Hz}, 1 \mathrm{H}), 7.42-7.34(\mathrm{~m}, 1 \mathrm{H}), 6.83(\mathrm{dd}$, $2 \mathrm{j}$ $J=7.6,1.2 \mathrm{~Hz}, 1 \mathrm{H}), 2.97-2.85(\mathrm{~m}, 1 \mathrm{H}), 2.85-2.81(\mathrm{~m}, 1 \mathrm{H}), 2.58-2.28(\mathrm{~m}, 5 \mathrm{H}), 2.18-2.03(\mathrm{~m}$, $2 \mathrm{H}), 1.04(\mathrm{~d}, \mathrm{~J}=7.2 \mathrm{~Hz}, 3 \mathrm{H})$; minor $\delta 8.10(\mathrm{dd}, J=7.6,1.2 \mathrm{~Hz}, 1 \mathrm{H}), 7.61(\mathrm{td}, J=7.6,1.6 \mathrm{~Hz}, 1 \mathrm{H}), 7.42-7.34$ (m, 2H), 1.95-1.87 (m, 1H), $1.68(\mathrm{ddd}, J=14.4,5.2,4.0 \mathrm{~Hz}, 1 \mathrm{H}), 3.01$ (q, $J=6.8 \mathrm{~Hz}, 1 \mathrm{H}), 2.80-2.76(\mathrm{~m}, 1 \mathrm{H})$, 2.76-2.68 (m, 1H), 2.58-2.28 (m, 3H), 2.18-2.03 (m, 1H), $1.00(\mathrm{~d}, J=7.2 \mathrm{~Hz}, 3 \mathrm{H}) ;{ }^{13} \mathrm{C}-\mathrm{NMR}(101 \mathrm{MHz}$, $\mathrm{CDCl}_{3}$ ) major $\delta 219.6,197.30,145.6,133.4,132.4,128.8,127.27,126.7,55.3,46.4,36.8,35.1,34.9,34.4$, 9.3; minor 217.7, 197.33, 147.4, 134.4, 132.9, 128.2, 127.33, 124.5, 53.7, 45.7, 34.7, 34.4, 32.8, 25.5, 7.7; IR (neat) $1738,1683 \mathrm{~cm}^{-1}$; HRMS (DART) $\mathrm{m} / z$ : $[\mathrm{M}+\mathrm{H}]^{+}$Calcd for $\mathrm{C}_{15} \mathrm{H}_{17} \mathrm{O}_{2} 229.1229$; Found 229.1229.

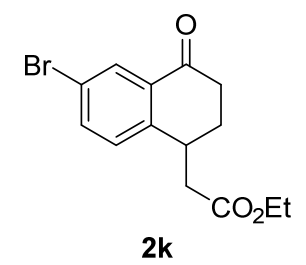

According to the representative procedure, $2 \mathbf{k}$ was obtained in $69 \%$ yield as a yellow oil after purification by silica gel column chromatography $($ Hexane/EtOAc $=10: 1)$. Analytical data for $2 \mathrm{k}:{ }^{1} \mathrm{H}-\mathrm{NMR}\left(400 \mathrm{MHz}, \mathrm{CDCl}_{3}\right) \delta 8.16(\mathrm{~d}, J=2.4 \mathrm{~Hz}, 1 \mathrm{H}), 7.61(\mathrm{dd}$, $J=8.0,2.4 \mathrm{~Hz}, 1 \mathrm{H}), 7.21(\mathrm{~d}, J=8.0 \mathrm{~Hz}, 1 \mathrm{H}), 4.18(\mathrm{q}, J=7.2 \mathrm{~Hz}, 2 \mathrm{H}), 3.53-3.47(\mathrm{~m}, 1 \mathrm{H})$, $2.81-2.61(\mathrm{~m}, 4 \mathrm{H}), 2.33-2.25(\mathrm{~m}, 1 \mathrm{H}), 2.11-2.03(\mathrm{~m}, 1 \mathrm{H}), 1.27(\mathrm{t}, J=7.2 \mathrm{~Hz}, 3 \mathrm{H}) ;{ }^{13} \mathrm{C}-$ NMR (101 MHz, $\left.\mathrm{CDCl}_{3}\right) \delta$ 196.3, 171.8, 144.9, 136.6, 133.6, 130.5, 129.8, 121.5, 61.0, 39.6, 35.0, 34.4, 27.4, 14.3; IR (neat) $1732,1687 \mathrm{~cm}^{-1}$; HRMS (DART) $\mathrm{m} / z$ : $\left[\mathrm{M}+\mathrm{NH}_{4}\right]^{+}$Calcd for $\mathrm{C}_{14} \mathrm{H}_{19} \mathrm{BrNO}_{3} 328.0548$; Found 328.0554 . 


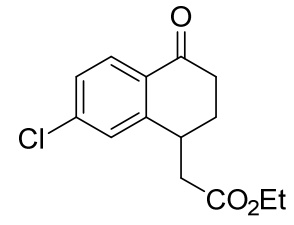

2l

According to the representative procedure, $\mathbf{2 l}$ was obtained in $77 \%$ yield as a yellow oil after purification by silica gel column chromatography (Hexane/EtOAc $=10: 1)$. Analytical data for 2l: ${ }^{1} \mathrm{H}-\mathrm{NMR}\left(400 \mathrm{MHz}, \mathrm{CDCl}_{3}\right) \delta 7.98(\mathrm{~d}, J=9.2 \mathrm{~Hz}, 1 \mathrm{H}), 7.33-7.30$ $(\mathrm{m}, 2 \mathrm{H}), 4.20$ (q, $J=7.2 \mathrm{~Hz}, 2 \mathrm{H}), 3.54-3.48(\mathrm{~m}, 1 \mathrm{H}), 2.80-2.72(\mathrm{~m}, 2 \mathrm{H}), 2.69-2.60(\mathrm{~m}$, 2H), 2.34-2.26 (m, 1H), 2.11-2.04 (m, 1H), $1.28(\mathrm{t}, J=7.2 \mathrm{~Hz}, 3 \mathrm{H}) ;{ }^{13} \mathrm{C}-\mathrm{NMR}(101 \mathrm{MHz}$, $\left.\mathrm{CDCl}_{3}\right) \delta 196.5,171.7,147.8,140.2,130.5,129.4,127.9,127.8,61.0,39.5,35.1,34.8,27.4,14.3$; IR (neat) 1732, $1687 \mathrm{~cm}^{-1}$; HRMS (DART) $\mathrm{m} / z$ : $\left[\mathrm{M}+\mathrm{NH}_{4}\right]^{+}$Calcd for $\mathrm{C}_{14} \mathrm{H}_{19} \mathrm{ClNO}_{3} 284.1054$; Found 284.1062.

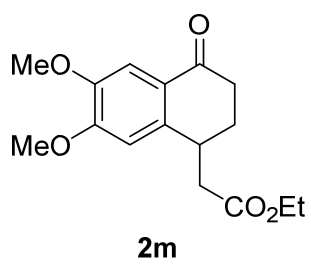

The reaction of $\mathbf{1 m}$ was performed for $2 \mathrm{~h} . \mathbf{2 m}$ was obtained in $79 \%$ yield as a yellow solid after purification by silica gel column chromatography (Hexane/EtOAc $=5: 1$ ). Analytical data for $2 \mathrm{~m}$ : $\left(\mathrm{mp} 84.6-85.5^{\circ} \mathrm{C}\right) ;{ }^{1} \mathrm{H}-\mathrm{NMR}\left(400 \mathrm{MHz}, \mathrm{CDCl}_{3}\right) \delta 7.52(\mathrm{~s}, 1 \mathrm{H})$, $6.75(\mathrm{~s}, 1 \mathrm{H}), 4.18(\mathrm{q}, J=7.2 \mathrm{~Hz}, 2 \mathrm{H}), 3.93(\mathrm{~s}, 3 \mathrm{H}), 3.92(\mathrm{~s}, 3 \mathrm{H}), 3.51-3.45(\mathrm{~m}, 1 \mathrm{H})$, $2.77-2.66(\mathrm{~m}, 3 \mathrm{H}), 2.64-2.56(\mathrm{~m}, 1 \mathrm{H}), 2.35-2.26(\mathrm{~m}, 1 \mathrm{H}), 2.09-2.02(\mathrm{~m}, 1 \mathrm{H}), 1.28(\mathrm{t}, J$ $=7.2 \mathrm{~Hz}, 3 \mathrm{H}) ;{ }^{13} \mathrm{C}-\mathrm{NMR}\left(101 \mathrm{MHz}, \mathrm{CDCl}_{3}\right) \delta 196.5,172.2,153.8,148.3,141.2,125.4,109.6,108.8,60.8$, 56.2, 56.1, 39.9, 34.7, 34.4, 27.8, 14.3; IR (KBr) 1731, $1660 \mathrm{~cm}^{-1}$; HRMS (DART) $m / z:[\mathrm{M}+\mathrm{H}]^{+}$Calcd for $\mathrm{C}_{16} \mathrm{H}_{22} \mathrm{O}_{5}$ 293.1389; Found 293.1361.

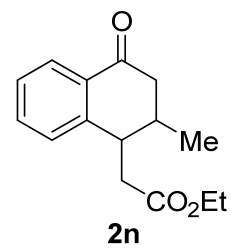

The reaction of $\mathbf{1 n}$ was performed for $2 \mathrm{~h} . \mathbf{2 n}$ was obtained in $77 \%$ yield as a yellow oil after purification by silica gel column chromatography $($ Hexane/EtOAc $=10: 1)$. Analytical data for $2 \mathrm{n}:{ }^{1} \mathrm{H}-\mathrm{NMR}\left(400 \mathrm{MHz}, \mathrm{CDCl}_{3}\right) \delta 8.02-8.01$ ( $\mathrm{m}, 1 \mathrm{H}$, major), 7.99 (m, 0.5H, minor), 7.50 (dt, $J=7.2,1.2 \mathrm{~Hz}, 0.5 \mathrm{H}$, minor), 7.46 (dt, $J=7.6,1.6 \mathrm{~Hz}, 1 \mathrm{H}$, major), 7.34-7.27 (m, 3H, major + minor), 4.18-4.07 (m, 3H, major + minor), 3.53-3.49 (m, 1H, major), 3.31-3.27 (m, 0.5H, minor), 2.88 (dd, $J=17.4,4.6 \mathrm{~Hz}, 0.5 \mathrm{H}$, minor), $2.77-2.36(\mathrm{~m}, 7 \mathrm{H}$, major + minor), $1.23(\mathrm{t}, J=7.2 \mathrm{~Hz}$, $1.5 \mathrm{H}$, minor), 1.19 ( $\mathrm{t}, J=7.3 \mathrm{~Hz}, 3 \mathrm{H}$, major), 1.07 (d, $J=6.4 \mathrm{~Hz}, 3 \mathrm{H}$, major), 1.05 (d, $J=6.4 \mathrm{~Hz}, 1.5 \mathrm{H}$, minor); ${ }^{13} \mathrm{C}-\mathrm{NMR}$ (101 MHz, $\mathrm{CDCl}_{3}$ ) $\delta 197.8$ (major), 197.3 (minor), 172.6 (major), 171.9 (minor), 146.7 (major), 144.5 (minor), 134.1 (minor), 133.7 (major), 131.4 (major + minor), 129.3 (minor), 128.3 (major), 127.5 (major), 127.3 (major), 127.2 (minor), 127.0 (minor), 60.7 (major + minor), 42.6 (major), 41.6 (minor), 41.4 (minor), 40.9 (minor), 40.1 (major), 35.6 (major), 32.6 (major), 32.5 (minor), 20.1 (minor), 17.6 (major), 14.2 (minor), 14.1 (major); IR (neat) 1732, $1685 \mathrm{~cm}^{-1}$; HRMS (DART) $\mathrm{m} / z$ : $\left[\mathrm{M}+\mathrm{NH}_{4}\right]^{+}$Calcd for $\mathrm{C}_{15} \mathrm{H}_{22} \mathrm{NO}_{3}$ 264.1600; Found 264.1615.

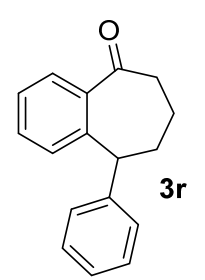

The reaction of $1 \mathbf{r}$ was performed at $30{ }^{\circ} \mathrm{C} .3 \mathbf{r}$ was obtained in $51 \%$ yield as a white amorphas solid after purification by silica gel column chromatography $($ Hexane/EtOAc $=10: 1)$. Analytical data for 3r: ${ }^{1} \mathrm{H}-\mathrm{NMR}\left(400 \mathrm{MHz}, \mathrm{CDCl}_{3}\right) \delta 7.65(\mathrm{dd}, J=7.2,1.6 \mathrm{~Hz}, 1 \mathrm{H}), 7.35(\mathrm{~d}, J=7.6 \mathrm{~Hz}$, 2H), 7.37-7.27 (m, 3H), $7.22(\mathrm{~d}, J=7.6 \mathrm{~Hz}, 2 \mathrm{H}), 6.83(\mathrm{dd}, J=7.2,1.6 \mathrm{~Hz}, 1 \mathrm{H}), 4.41(\mathrm{dd}, J=$ $10.4,4.0 \mathrm{~Hz}, 1 \mathrm{H}), 2.82-2.66(\mathrm{~m}, 2 \mathrm{H}), 2.38-2.30(\mathrm{~m}, 1 \mathrm{H}), 2.23-2.15(\mathrm{~m}, 1 \mathrm{H}), 2.03-1.96(\mathrm{~m}, 1 \mathrm{H})$, 1.77-1.71 (m, 1H); ${ }^{13} \mathrm{C}-\mathrm{NMR}\left(101 \mathrm{MHz}, \mathrm{CDCl}_{3}\right) \delta 207.4,142.9,142.3,139.7,131.9,129.0,128.8,128.7$, 128.3, 126.8, 47.1, 41.1, 31.5, 20.6, One Csp ${ }^{2}$ signal is missing due to overlapping.; IR (KBr) $1672 \mathrm{~cm}^{-1}$; 
HRMS (DART) $m / z:\left[\mathrm{M}+\mathrm{NH}_{4}\right]^{+}$Calcd for $\mathrm{C}_{17} \mathrm{H}_{20} \mathrm{NO} 254.1545$; Found 254.1518.

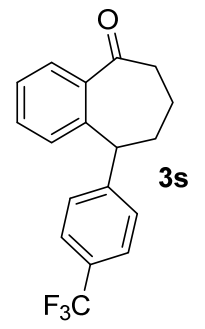

The reaction of $1 \mathrm{~s}$ was performed at $30^{\circ} \mathrm{C}$. $3 \mathrm{~s}$ was obtained in $91 \%$ yield as a yellow amorphas solid after purification by silica gel column chromatography $($ Hexane/EtOAc $=10: 1)$. Analytical data for 3s: ${ }^{1} \mathrm{H}-\mathrm{NMR}\left(400 \mathrm{MHz}, \mathrm{CDCl}_{3}\right) \delta 7.66(\mathrm{dd}, J=7.2,1.6 \mathrm{~Hz}, 1 \mathrm{H}), 7.61(\mathrm{~d}, J=8.4 \mathrm{~Hz}$, 2H), 7.37-7.30 (m, 4H), $6.77(\mathrm{~d}, J=7.2 \mathrm{~Hz}, 1 \mathrm{H}), 4.46(\mathrm{dd}, J=10.0,4.0 \mathrm{~Hz}, 1 \mathrm{H}), 2.82-2.66(\mathrm{~m}$, 2H), 2.40-2.31 (m, 1H), 2.25-2.16 (m, 1H), 2.06-1.97 (m, 1H), 1.81-1.67 (m, 1H); ${ }^{13} \mathrm{C}-\mathrm{NMR}$ $\left(101 \mathrm{MHz}, \mathrm{CDCl}_{3}\right) \delta 206.9,146.6,141.7,139.9,132.0,129.2$ (q, $\left.J=32 \mathrm{~Hz}\right), 129.1,128.8,128.5$, 127.2, $125.6(\mathrm{q}, J=3.8 \mathrm{~Hz}), 47.2,41.1,31.4,20.5$, A signal of $C \mathrm{~F}_{3}$ is obscure due to overlapping with aromatic peaks.; ${ }^{19} \mathrm{~F}-\mathrm{NMR}\left(376 \mathrm{MHz}, \mathrm{CDCl}_{3}\right) \delta-63.4$; IR $(\mathrm{KBr}) 1673 \mathrm{~cm}^{-1}$; HRMS (DART) $m / z$ : $[\mathrm{M}+\mathrm{H}]^{+} \mathrm{Calcd}$ for $\mathrm{C}_{18} \mathrm{H}_{16} \mathrm{~F}_{3} \mathrm{O} 305.1153$; Found 305.1179.

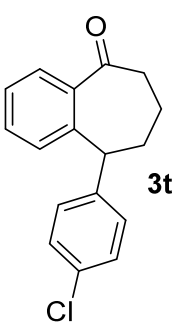

The reaction of $\mathbf{1 t}$ was performed at $30{ }^{\circ} \mathrm{C} .{ }^{1} \mathrm{H}$ NMR analysis of the crude mixture showed $3 \mathrm{t}: 2 \mathrm{t}$ $=11: 1.3 \mathrm{t}$ was purified by silica gel column chromatography $($ Hexane/EtOAc $=10: 1)$ and obtained in $71 \%$ yield (pale-yellow amorphas solid) as a mixture with $9 \%$ of 1 -tetralone product $2 \mathbf{t}$ which was inseparable from 3t. Analytical data for 3t: ${ }^{1} \mathrm{H}-\mathrm{NMR}\left(400 \mathrm{MHz}, \mathrm{CDCl}_{3}\right) \delta 7.65$ $(\mathrm{dd}, J=7.2,1.6 \mathrm{~Hz}, 1 \mathrm{H}), 7.36-7.28(\mathrm{~m}, 4 \mathrm{H}), 7.15(\mathrm{~d}, J=8.0 \mathrm{~Hz}, 2 \mathrm{H}), 6.79$ (dd, $J=7.2,1.6 \mathrm{~Hz}$, 1H), 4.38 (dd, $J=10.4,4.4 \mathrm{~Hz}, 1 \mathrm{H}), 2.81-2.74(\mathrm{~m}, 1 \mathrm{H}), 2.73-2.65(\mathrm{~m}, 1 \mathrm{H}), 2.34-2.25(\mathrm{~m}, 1 \mathrm{H})$, 2.22-2.13 (m, 1H), 2.04-1.96 (m, 1H), 1.79-1.68 (m, 1H); ${ }^{13} \mathrm{C}-\mathrm{NMR}\left(101 \mathrm{MHz}, \mathrm{CDCl}_{3}\right) \delta 207.1,142.2,140.9$, 139.7, 132.6, 131.9, 130.1, 128.82, 128.79, 128.4, 127.1, 46.6, 41.1, 31.4, 20.5; IR (neat) $1683 \mathrm{~cm}^{-1}$; HRMS (DART) $m / z:\left[\mathrm{M}+\mathrm{NH}_{4}\right]^{+}$Calcd for $\mathrm{C}_{17} \mathrm{H}_{19} \mathrm{ClNO} 288.1155$; Found 288.1142 .

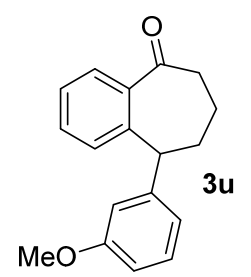

The reaction of $1 \mathbf{u}$ was performed at $30{ }^{\circ} \mathrm{C} .{ }^{1} \mathrm{H}$ NMR analysis of the crude mixture showed $3 \mathbf{u}: 2 \mathbf{u}=20: 1.3 \mathbf{u}$ was purified by silica gel column chromatography (Hexane/EtOAc $=10: 1)$ and obtained in 34\% yield (white amorphas solid) as a mixture with 5\% of 1-tetralone product 2u which was inseparable from 3u. ${ }^{1} \mathrm{H}$ NMR analysis of the crude product mixture showed that $58 \%$ of 1 s was recovered. Analytical data for $3 \mathrm{u}:{ }^{1} \mathrm{H}-\mathrm{NMR}\left(400 \mathrm{MHz}, \mathrm{CDCl}_{3}\right) \delta 7.64$ $(\mathrm{dd}, J=7.2,1.6 \mathrm{~Hz}, 1 \mathrm{H}), 7.33(\mathrm{td}, J=7.2,1.6 \mathrm{~Hz}, 1 \mathrm{H}), 7.31-7.29(\mathrm{~m}, 1 \mathrm{H}), 7.26(\mathrm{~d}, J=7.2 \mathrm{~Hz}, 1 \mathrm{H}), 6.86(\mathrm{dd}$, $J=7.2,1.2 \mathrm{~Hz}, 1 \mathrm{H}), 6.83(\mathrm{t}, J=2.0 \mathrm{~Hz}, 1 \mathrm{H}), 6.81(\mathrm{t}, J=2.0 \mathrm{~Hz}, 1 \mathrm{H}), 6.76(\mathrm{t}, J=2.0 \mathrm{~Hz}, 1 \mathrm{H}), 4.37(\mathrm{dd}, J=$ 10.8, $4.0 \mathrm{~Hz}, 1 \mathrm{H}), 3.78(\mathrm{~s}, 3 \mathrm{H}), 2.83-2.76(\mathrm{~m}, 1 \mathrm{H}), 2.73-2.65(\mathrm{~m}, 1 \mathrm{H}), 2.36-2.27(\mathrm{~m}, 1 \mathrm{H}), 2.23-2.14(\mathrm{~m}, 1 \mathrm{H})$, 2.04-1.95 (m, 1H), 1.79-1.69 (m, 1H); ${ }^{13} \mathrm{C}-\mathrm{NMR}\left(101 \mathrm{MHz}, \mathrm{CDCl}_{3}\right) \delta$ 207.3, 159.9, 144.0, 142.7, 139.7, 131.9, 129.6, 129.0, 128.3, 126.9, 121.1, 114.8, 111.9, 55.3, 47.1, 41.1, 31.4, 20.6; IR (neat) $1678 \mathrm{~cm}^{-1}$; HRMS (DART) $m / z:[\mathrm{M}+\mathrm{H}]^{+}$Calcd for $\mathrm{C}_{18} \mathrm{H}_{19} \mathrm{O}_{2}$ 267.1385; Found 267.1375. 


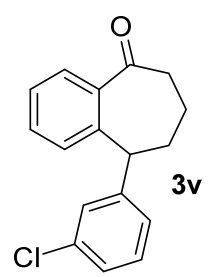

The reaction of $1 \mathbf{v}$ was performed at $30{ }^{\circ} \mathrm{C} . \mathbf{3 v}$ was obtained in $87 \%$ yield as a pale-yellow amorphas solid after purification by silica gel column chromatography $(\mathrm{Hexane} / \mathrm{EtOAc}=10: 1)$. Analytical data for 3v: ${ }^{1} \mathrm{H}-\mathrm{NMR}\left(400 \mathrm{MHz}, \mathrm{CDCl}_{3}\right) \delta 7.65(\mathrm{dd}, J=7.6,1.2 \mathrm{~Hz}, 1 \mathrm{H}), 7.37-$ $7.23(\mathrm{~m}, 5 \mathrm{H}), 7.08(\mathrm{td}, J=6.8,2.0 \mathrm{~Hz}, 1 \mathrm{H}), 6.80(\mathrm{dd}, J=7.6,1.2 \mathrm{~Hz}, 1 \mathrm{H}), 4.38(\mathrm{dd}, J=10.8$, $4.4 \mathrm{~Hz}, 1 \mathrm{H}), 2.75-2.81(\mathrm{~m}, 1 \mathrm{H}), 2.66-2.73(\mathrm{~m}, 1 \mathrm{H}), 2.26-2.34(\mathrm{~m}, 1 \mathrm{H}), 2.13-2.22(\mathrm{~m}, 1 \mathrm{H})$, 1.96-2.04 (m, 1H), 1.68-1.81 (m, 1H); ${ }^{13} \mathrm{C}-\mathrm{NMR}\left(101 \mathrm{MHz}, \mathrm{CDCl}_{3}\right) \delta$ 206.9, 144.5, 142.0, 139.7, 134.6, 132.0, $129.9,128.8,128.5,127.11,127.07,127.04,46.9,41.1,31.3,20.5$, One Csp ${ }^{2}$ signal is missing due to overlapping.; IR (neat) $1679 \mathrm{~cm}^{-1}$; HRMS (DART) $\mathrm{m} / z$ : [M $\left.+\mathrm{NH}_{4}\right]^{+}$Calcd for $\mathrm{C}_{17} \mathrm{H}_{19} \mathrm{ClNO} 288.1155$; Found 288.1146.

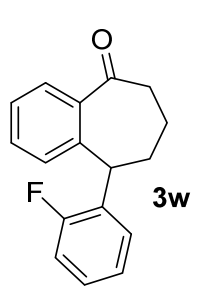

The reaction of $1 \mathrm{w}$ was performed at $30^{\circ} \mathrm{C} .3 \mathrm{w}$ was obtained in $66 \%$ yield as a colorless oil after purification by silica gel column chromatography (Hexane/EtOAc $=20: 1) .3 \mathbf{w}$ was analyzed as a mixture containing a trace amount of 1-tetralone product $\mathbf{2 w}$ which was inseparable from $\mathbf{3 w}$.

Analytical data for 3w: ${ }^{1} \mathrm{H}-\mathrm{NMR}\left(400 \mathrm{MHz}, \mathrm{CDCl}_{3}\right) \delta 7.67(\mathrm{dd}, J=7.2,1.6 \mathrm{~Hz}, 1 \mathrm{H}), 7.35-$ $7.27(\mathrm{~m}, 3 \mathrm{H}), 7.23(\mathrm{td}, J=7.6,1.2 \mathrm{~Hz}, 1 \mathrm{H}), 7.15(\mathrm{td}, J=7.6,1.2 \mathrm{~Hz}, 1 \mathrm{H}), 7.09-7.04(\mathrm{~m}, 1 \mathrm{H})$, 6.82-6.80 (m, 1H), $4.65(\mathrm{dd}, J=10.8,4.0 \mathrm{~Hz}, 1 \mathrm{H}), 2.86-2.72(\mathrm{~m}, 2 \mathrm{H}), 2.38-2.33(\mathrm{~m}, 1 \mathrm{H}), 2.17-2.09(\mathrm{~m}, 1 \mathrm{H})$, 2.06-1.99 (m, 1H), 1.77-1.71 (m, 1H); ${ }^{13} \mathrm{C}-\mathrm{NMR}\left(101 \mathrm{MHz}, \mathrm{CDCl}_{3}\right) \delta 206.9,160.9$ (d, $\left.J=247 \mathrm{~Hz}\right), 141.8$, 139.7, 132.0, 130.0 (d, $J=4.8 \mathrm{~Hz}), 129.6$ (d, $J=14.4 \mathrm{~Hz}), 128.6$ (d, $J=7.7 \mathrm{~Hz}), 128.5,128.1,127.0,124.3$ $(\mathrm{d}, J=3.9 \mathrm{~Hz}), 115.8(\mathrm{~d}, J=22.1 \mathrm{~Hz}), 41.2,40.9,30.8,20.7 ;{ }^{19} \mathrm{~F}-\mathrm{NMR}\left(376 \mathrm{MHz}, \mathrm{CDCl}_{3}\right) \delta-116.3$; IR (neat) $1680 \mathrm{~cm}^{-1}$; HRMS (DART) $\mathrm{m} / z$ : $[\mathrm{M}+\mathrm{H}]^{+}$Calcd for $\mathrm{C}_{17} \mathrm{H}_{16} \mathrm{FO} 255.1185$; Found 255.1184.

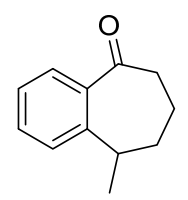

The reaction was performed at $30{ }^{\circ} \mathrm{C} .3 \mathbf{x}$ was obtained in $42 \%$ yield as a colorless oil after purification by silica gel column chromatography (Hexane/EtOAc $=20: 1) .{ }^{1} \mathrm{H}$ NMR analysis of the crude product mixture showed that $\mathbf{1} \mathbf{x}$ was not detected and the crude mixture was dirty. 3x Spectra are consistent with reported literature values. ${ }^{16}$ Analytical data for 3x: ${ }^{1} \mathrm{H}-\mathrm{NMR}$ (400 $\left.\mathrm{MHz}, \mathrm{CDCl}_{3}\right) \delta 7.54(\mathrm{dd}, J=7.6,1.6 \mathrm{~Hz}, 1 \mathrm{H}), 7.49-7.27(\mathrm{~m}, 3 \mathrm{H}), 3.17-3.06(\mathrm{~m}, 1 \mathrm{H}), 2.79-2.52(\mathrm{~m}, 2 \mathrm{H})$, 2.01-1.86 (m, 2H), 1.66-1.47 (m, 2H), 1.39 (d, $J=6.8 \mathrm{~Hz}, 3 \mathrm{H}) ;{ }^{13} \mathrm{C}-\mathrm{NMR}\left(101 \mathrm{MHz}, \mathrm{CDCl}_{3}\right) \delta 208.7,143.3$, 139.6, 132.1, 128.0, 126.6, 125.4, 41.3, 34.5, 34.3, 20.5, 19.4.

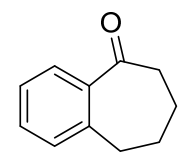

The reaction was performed at $30{ }^{\circ} \mathrm{C} .3 \mathbf{y}$ was obtained in $62 \%$ yield as a colorless oil after purification by silica gel column chromatography (Hexane/EtOAc $=20: 1)$. Spectra are consistent with reported literature values. ${ }^{17}$ Analytical data for 3y: ${ }^{1} \mathrm{H}-\mathrm{NMR}\left(400 \mathrm{MHz}, \mathrm{CDCl}_{3}\right) \delta 7.72(\mathrm{dd}$, 3y $J=7.6,1.6 \mathrm{~Hz}, 1 \mathrm{H}), 7.42(\mathrm{td}, J=7.6,1.6 \mathrm{~Hz}, 1 \mathrm{H}), 7.30(\mathrm{td}, J=7.6,1.6 \mathrm{~Hz}, 1 \mathrm{H}), 7.20(\mathrm{~d}, J=7.6$ $\mathrm{Hz}, 1 \mathrm{H}), 2.93(\mathrm{t}, J=6.0 \mathrm{~Hz}, 2 \mathrm{H}), 2.75-2.72(\mathrm{~m}, 2 \mathrm{H}), 1.92-1.78(\mathrm{~m}, 4 \mathrm{H}) ;{ }^{13} \mathrm{C}-\mathrm{NMR}\left(101 \mathrm{MHz}, \mathrm{CDCl}_{3}\right) \delta 206.3$, $141.4,138.9,132.3,129.8,128.7,126.7,40.9,32.6,25.3,21.0$. 


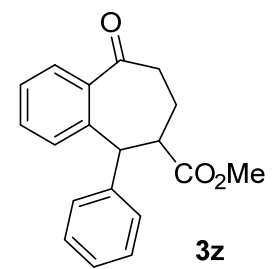

According to the representative procedure, $\mathbf{3 z}$ was obtained in $78 \%$ yield as a colorless oil after purification by silica gel column chromatography (Hexane/EtOAc $=10: 1) .3 z$ was analyzed as a mixture of diastereomers (major/minor $=14: 1$ ). Analytical data for $\mathbf{3 z}$ (major diastereomer (cis-isomer)): ${ }^{1} \mathrm{H}-\mathrm{NMR}\left(400 \mathrm{MHz}, \mathrm{CDCl}_{3}\right) \delta 7.79(\mathrm{dd}, J=8.0,2.0$ $\mathrm{Hz}, 1 \mathrm{H}), 7.46(\mathrm{td}, J=8.0,2.0 \mathrm{~Hz}, 1 \mathrm{H}), 7.38(\mathrm{td}, J=8.0,2.0 \mathrm{~Hz}, 1 \mathrm{H}), 7.32-7.22(\mathrm{~m}, 4 \mathrm{H})$, $7.00(\mathrm{~d}, J=8.0 \mathrm{~Hz}, 2 \mathrm{H}), 5.18(\mathrm{~s}, 1 \mathrm{H}), 3.81(\mathrm{~s}, 3 \mathrm{H}), 3.27$ (ddd, $J=12.8,5.2,2.0 \mathrm{~Hz}, 1 \mathrm{H}), 2.90-2.82(\mathrm{~m}, 1 \mathrm{H})$, 2.59-2.53 (m, 1H), 2.27-2.19 (m, 1H), 1.99-1.90 (m, 1H); ${ }^{13} \mathrm{C}-\mathrm{NMR}\left(101 \mathrm{MHz}, \mathrm{CDCl}_{3}\right) \delta$ 204.3, 174.5, 141.9, 139.9, 139.5, 131.94, 131.86, 129.3, 128.6, 128.5, 127.4, 127.0, 52.4, 51.0, 47.4, 40.4, 22.3; IR (neat) 1733, $1682 \mathrm{~cm}^{-1}$; HRMS (DART) $\mathrm{m} / z$ : $\left[\mathrm{M}+\mathrm{NH}_{4}\right]^{+}$Calcd for $\mathrm{C}_{19} \mathrm{H}_{22} \mathrm{NO} 312.1600$; Found 312.1596.

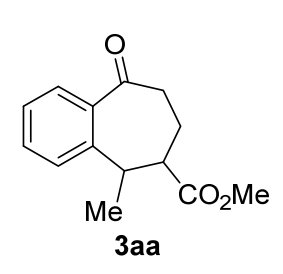

${ }^{1} \mathrm{H}$ NMR analysis of the crude mixture indicated that 3aa and $2 \mathbf{a a}$ were obtained in $42 \%$ $(2: 1 \mathrm{dr})$ and $21 \%$ NMR yields, respectively. Isolation of the minor diastereomer of $\mathbf{3 a a}$ for analysis was performed by silica gel column chromatography (Hexane/EtOAc $=5: 1)$ and the pure sample was obtained in $8 \%$ yield as a yellow oil. However, the major diastereomer of 3aa and 2aa could not be separated. Analytical data for 3aa (minor diastereomer): ${ }^{1} \mathrm{H}$ -

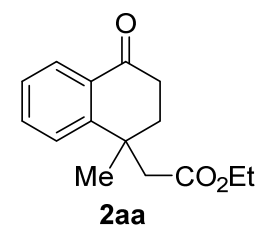
NMR (400 MHz, $\left.\mathrm{CDCl}_{3}\right) \delta$ 7.56-7.48 (m, 2H), 7.35-7.30 (m, 2H), 3.75 (s, 3H), 3.49-3.41 (m, 1H), 3.05 (ddd, $J=19.6,12.5,2.4 \mathrm{~Hz}, 1 \mathrm{H}), 2.67-2.53$ (m, 2H), 2.01-1.85 (m, 2H), 1.37 $(\mathrm{d}, J=6.9 \mathrm{~Hz}, 3 \mathrm{H}) ;{ }^{13} \mathrm{C}-\mathrm{NMR}\left(101 \mathrm{MHz}, \mathrm{CDCl}_{3}\right) \delta 207.4,175.5,140.8,139.1,132.3,128.0$, 127.3, 125.8, 51.9, 48.5, 37.8, 36.0, 24.5, 17.9; IR (neat) 1732, $1683 \mathrm{~cm}^{-1}$; HRMS (DART) $m / z:\left[\mathrm{M}+\mathrm{NH}_{4}\right]^{+}$Calcd for $\mathrm{C}_{14} \mathrm{H}_{20} \mathrm{NO}_{3}$ 250.1443; Found 250.1439. Analytical data for 3aa (major diastereomer) and 2aa: ${ }^{1} \mathrm{H}-\mathrm{NMR}\left(400 \mathrm{MHz}, \mathrm{CDCl}_{3}\right) \delta 8.05(\mathrm{dd}, J=8.0,1.6 \mathrm{~Hz}, 1 \mathrm{H}, 2 \mathbf{2 a a}), 7.56-7.54(\mathrm{~m}, 1 \mathrm{H}$, 3aa), 7.54-7.52 (m, 1H, 2aa), 7.48 (td, $J=7.6,1.5 \mathrm{~Hz}, 1 \mathrm{H}, 3 \mathbf{a a}), 7.39$ (d, $J=7.8 \mathrm{~Hz}, 1 \mathrm{H}, 3 \mathbf{a a}), 7.36-7.27$ (m, $3 \mathrm{H}, 3 \mathbf{a a}+2 \mathbf{a a}), 3.68(\mathrm{~s}, 3 \mathrm{H}, 3 \mathbf{a a}$ or 2aa), 3.63 (s, 3H, 3aa or 2aa), 3.46-3.39 (m, 1H, 3aa), 2.96 (dt, $J=11.8$, $5.3 \mathrm{~Hz}, 1 \mathrm{H}, \mathbf{3 a a}), 2.88-2.60$ (m, 7H, 3aa + 2aa), 2.38 (ddd, $J=13.9,7.0,5.4 \mathrm{~Hz}, 1 \mathrm{H}, \mathbf{2 a a}), 2.20-2.12(\mathrm{~m}, 1 \mathrm{H}$, 3aa), 2.08-1.93 (m, 2H, 3aa + 2aa), 1.52 (s, 3H, 2aa), 1.39 (d, $J=7.3 \mathrm{~Hz}, 3 \mathrm{H}, \mathbf{3 a a})$; ${ }^{13} \mathrm{C}-\mathrm{NMR}(101 \mathrm{MHz}$, $\left.\mathrm{CDCl}_{3}\right) \delta 206.8,197.7,174.2,171.3,149.9,141.0,139.2,133.9,131.9,131.3,127.9,127.6,127.0,126.9$, 125.8, 51.6, 51.5, 47.6, 45.2, 40.3, 37.2, 36.4, 34.7, 33.8, 27.4, 23.9, 16.7; IR (neat) 1733, $1683 \mathrm{~cm}^{-1}$; HRMS (DART) $m / z:\left[\mathrm{M}+\mathrm{NH}_{4}\right]^{+}$Calcd for $\mathrm{C}_{14} \mathrm{H}_{20} \mathrm{NO}_{3} 250.1443$; Found 250.1433 .

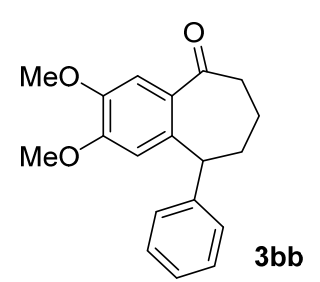

The reaction of $\mathbf{1 b b}$ was performed at $30{ }^{\circ} \mathrm{C}$ for $2 \mathrm{~h}$. $\mathbf{3 b b}$ was obtained in $77 \%$ yield as a yellow oil after purification by silica gel column chromatography $($ Hexane/EtOAc $=$ 10:1). Analytical data for 3bb: ${ }^{1} \mathrm{H}-\mathrm{NMR}\left(400 \mathrm{MHz}, \mathrm{CDCl}_{3}\right) \delta 7.38-7.34(\mathrm{~m}, 2 \mathrm{H}), 7.32$ (s, 1H), 7.29-7.25 (m, 1H), 7.23-7.21 (m, 2H), $6.33(\mathrm{~s}, 1 \mathrm{H}), 4.42$ (dd, $J=10.0,4.0 \mathrm{~Hz}$, $1 \mathrm{H}), 3.92(\mathrm{~s}, 3 \mathrm{H}), 3.67(\mathrm{~s}, 3 \mathrm{H}), 2.81-2.74(\mathrm{~m}, 1 \mathrm{H}), 2.71-2.63(\mathrm{~m}, 1 \mathrm{H}), 2.41-2.32(\mathrm{~m}, 1 \mathrm{H})$, 2.23-2.14 (m, 1H), 2.02-1.93 (m, 1H), 1.79-1.68 (m, 1H); ${ }^{13} \mathrm{C}-\mathrm{NMR}\left(101 \mathrm{MHz}, \mathrm{CDCl}_{3}\right) \delta 204.9,151.7,147.4$, 142.7, 138.1, 131.9, 128.7, 128.6, 126.8, 112.3, 111.3, 56.1, 55.8, 47.2, 41.3, 31.5, 20.4; IR (neat) $1667 \mathrm{~cm}^{-1}$; HRMS (DART) m/z: [M+ H] Calcd for $\mathrm{C}_{19} \mathrm{H}_{21} \mathrm{O}_{3}$ 297.1491; Found 297.1480. 


\section{Ring Expansion of the Alkyne Substrates 10 and 1p}

\section{Preparation of the alkyne substrates}

$\mathbf{1 0}$ and $\mathbf{1 p}$ was prepared according to the previous report. ${ }^{4}$

\section{characterization of 20 and $2 p$}

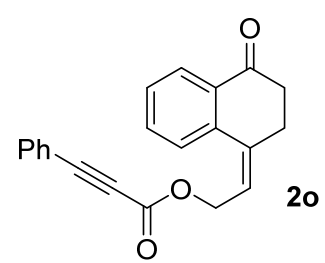

The reaction of 10 was performed at $30^{\circ} \mathrm{C} .20$ was obtained in $44 \%$ yield $(Z / E=10: 1)$ as a pale-yellow oil after purification by silica gel column chromatography $($ Hexane/EtOAc $=10: 1) .{ }^{1} \mathrm{H}$ NMR analysis of the crude product mixture showed that $53 \%$ of 10 was recovered. Analytical data for $20:{ }^{1} \mathrm{H}-\mathrm{NMR}\left(400 \mathrm{MHz}, \mathrm{CDCl}_{3}\right) \delta 8.11$ (dd, $J=7.6,1.2 \mathrm{~Hz}, 1 \mathrm{H}), 7.64-7.59(\mathrm{~m}, 3 \mathrm{H}), 7.50-7.44(\mathrm{~m}, 2 \mathrm{H}), 7.41-7.36(\mathrm{~m}, 3 \mathrm{H})$, $5.97(\mathrm{t}, J=6.8 \mathrm{~Hz}, 1 \mathrm{H}), 5.01(\mathrm{~d}, J=6.8 \mathrm{~Hz}, 2 \mathrm{H}), 2.82(\mathrm{br} \mathrm{s}, 4 \mathrm{H}){ }^{13} \mathrm{C}-\mathrm{NMR}\left(101 \mathrm{MHz}, \mathrm{CDCl}_{3}\right) \delta 197.3,153.9$, 140.3, 139.2, 133.5, 133.1, 132.5, 130.9, 129.1, 128.7, 128.0, 127.8, 120.8, 119.5, 87.0, 80.4, 63.3, 40.1, 35.1; IR (neat) 1685, $1708 \mathrm{~cm}^{-1}$; HRMS (DART) $\mathrm{m} / z$ : $\left[\mathrm{M}+\mathrm{NH}_{4}\right]^{+}$Calcd for $\mathrm{C}_{21} \mathrm{H}_{20} \mathrm{NO}_{3}$ 334.1443; Found 334.1459.

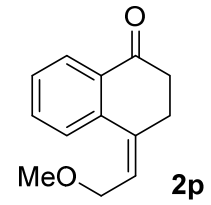

The reaction of $1 \mathbf{p}$ was performed at $30^{\circ} \mathrm{C}$. $2 \mathbf{p}$ was obtained as the $Z$-isomer in $56 \%$ yield as a pale-yellow oil after purification by silica gel column chromatography $($ Hexane/EtOAc $=10: 1)$. ${ }^{1} \mathrm{H}$ NMR analysis of the crude product mixture showed that $35 \%$ of $\mathbf{1 p}$ was recovered. Analytical data for $2 \mathrm{p:}{ }^{1} \mathrm{H}-\mathrm{NMR}\left(400 \mathrm{MHz}, \mathrm{CDCl}_{3}\right) \delta 8.08(\mathrm{dd}, J=7.6,1.2 \mathrm{~Hz}, 1 \mathrm{H}), 7.57$ (td, $J=7.6,1.2 \mathrm{~Hz}, 1 \mathrm{H}), 7.43(\mathrm{td}, J=7.6,1.2 \mathrm{~Hz}, 1 \mathrm{H}), 7.34(\mathrm{dd}, J=7.6,1.2 \mathrm{~Hz}, 1 \mathrm{H}), 5.93(\mathrm{t}, J=7.2 \mathrm{~Hz}, 1 \mathrm{H}), 4.17$ (d, $J=7.2 \mathrm{~Hz}, 2 \mathrm{H}), 3.40$ (s, 3H), 2.79 (br s, 4H); ${ }^{13} \mathrm{C}-\mathrm{NMR}\left(101 \mathrm{MHz}, \mathrm{CDCl}_{3}\right.$ ) $\delta 197.8,139.9,137.8,133.2$, 132.3, 128.5, 128.2, 127.5, 124.8, 69.6, 58.4, 40.4, 35.1; IR (neat) $1685 \mathrm{~cm}^{-1}$; HRMS (DART) $m / z:\left[\mathrm{M}+\mathrm{NH}_{4}\right]^{+}$ Calcd for $\mathrm{C}_{13} \mathrm{H}_{18} \mathrm{NO}_{2} 220.1338$; Found 220.1313 . 
The structure of $\mathbf{2 0}$ was assigned by NOESY experiments. The arrows shown below indicate the observed cross peaks.
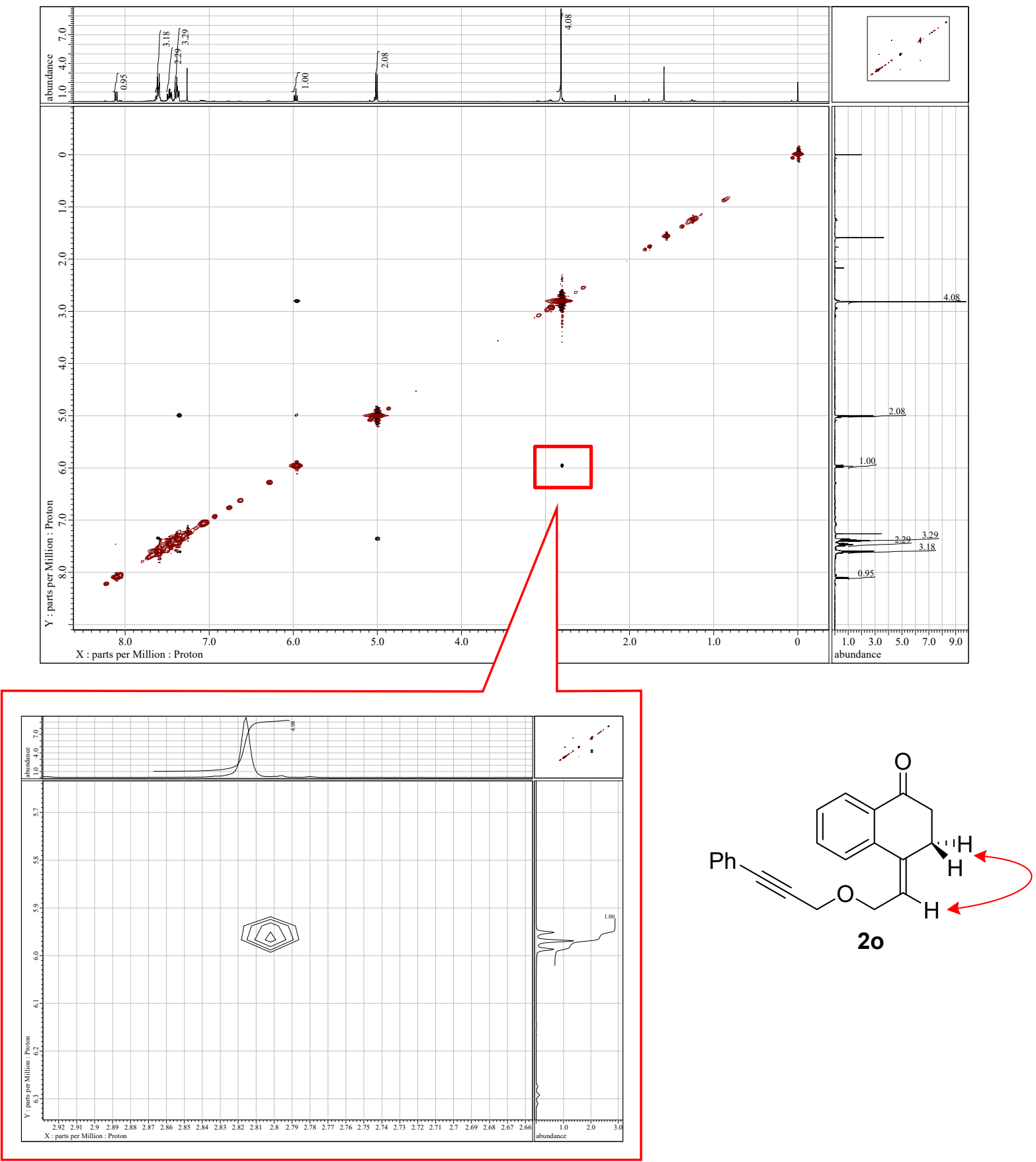

20 
The structure of $\mathbf{2 p}$ was assigned by NOESY experiments. The arrows shown below indicate the observed cross peaks.

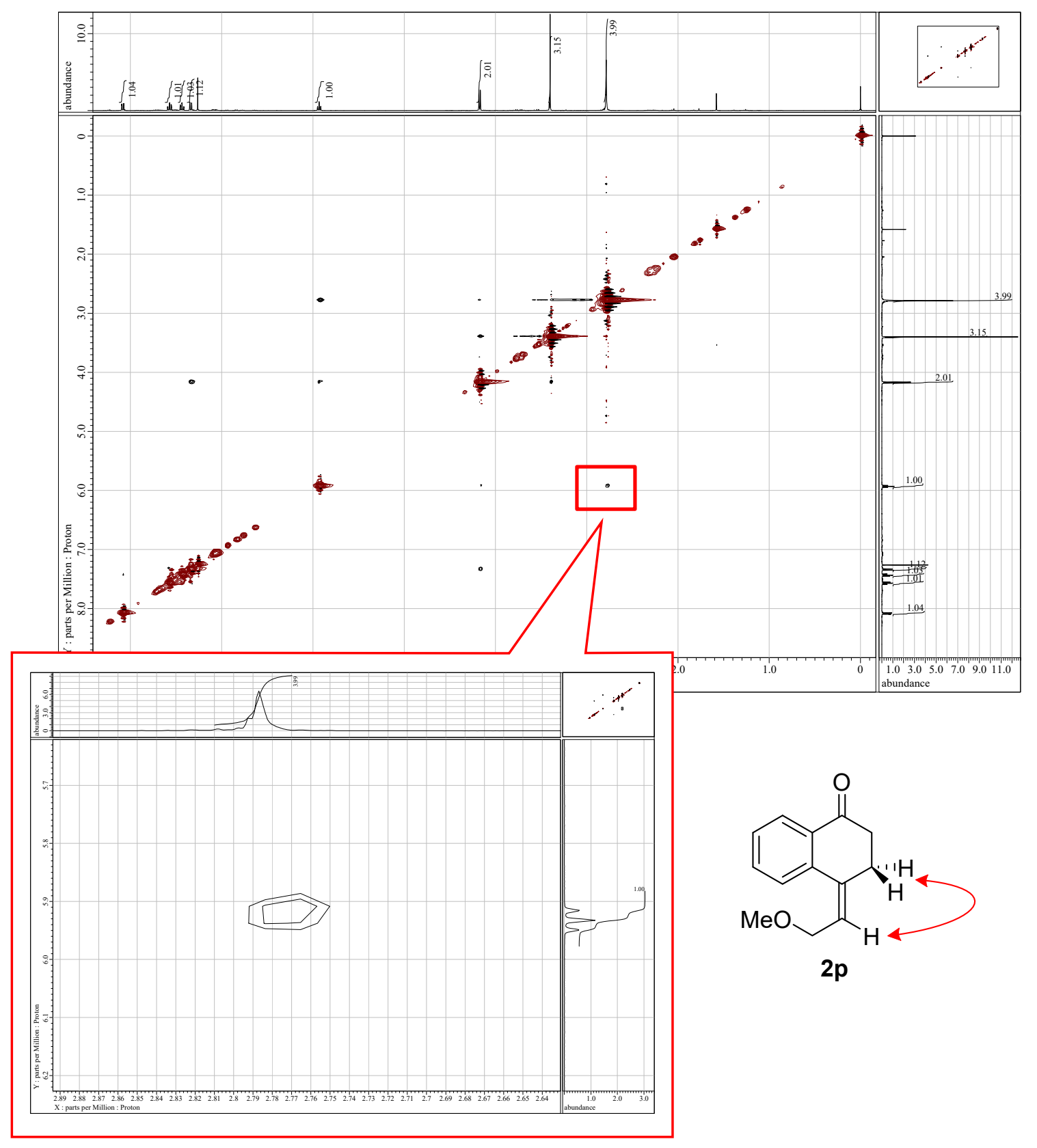




\section{Radical Cyclization of Cyclobutanol Substrate 5 and Cyclopentanol Substrate 6}

\section{Preparation of cyclobutanol 5}
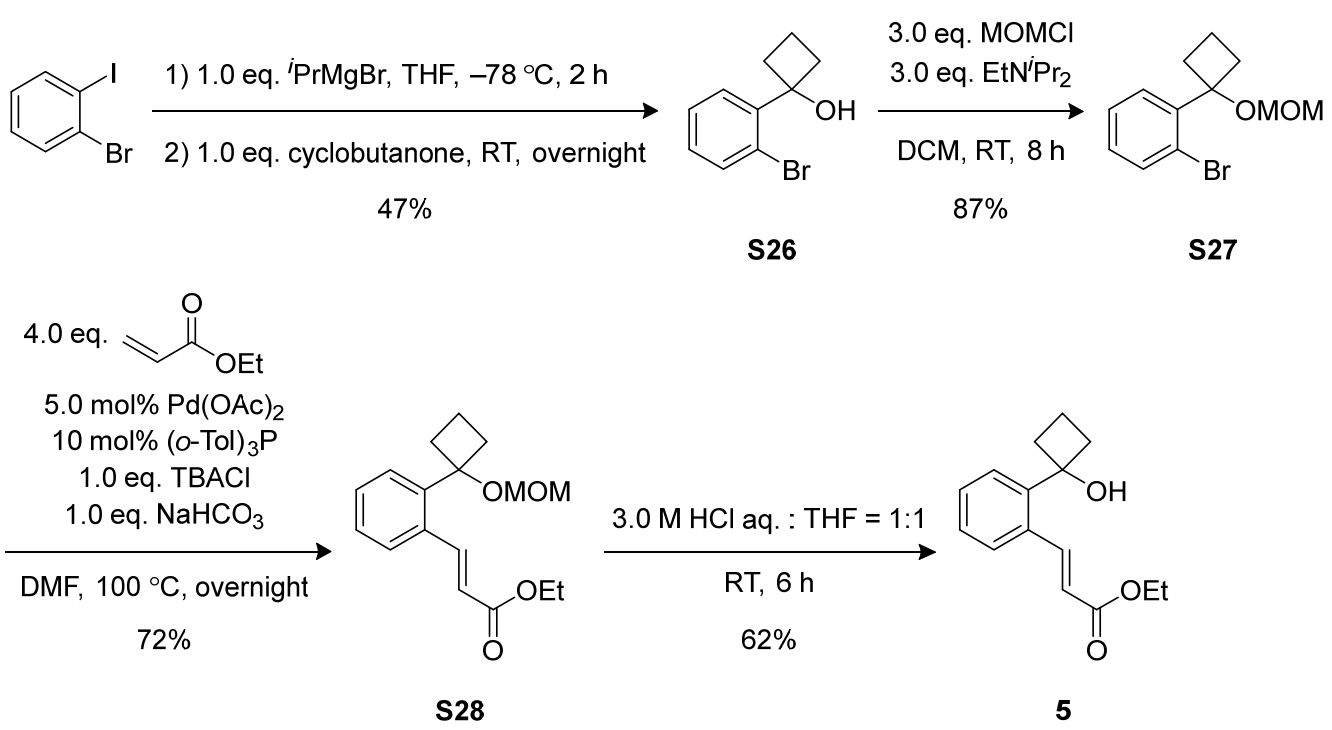

S26 was prepared according to the previous report. ${ }^{18}$

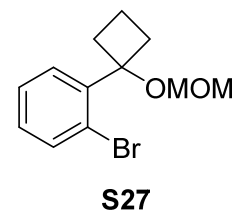

To a solution of $\mathbf{S 2 6}(500 \mathrm{mg}, 2.20 \mathrm{mmol})$ and $\operatorname{EtN}^{i} \operatorname{Pr}_{2}(1.10 \mathrm{~mL}, 6.35 \mathrm{mmol})$ in dry DCM $(10 \mathrm{~mL})$ was added $\mathrm{MOMCl}(500 \mu \mathrm{L}, 6.65 \mathrm{mmol})$ at $0{ }^{\circ} \mathrm{C}$, and the mixture was allowed to warm to rt. After being stirred at $\mathrm{rt}$ for $8 \mathrm{~h}$, the reaction was quenched with water and extracted with EtOAc. The organic layer was washed with brine, dried over $\mathrm{MgSO}_{4}$, and concentrated in vacuo. The residue was purified by silica gel column chromatography (Hexane/EtOAc $=20: 1)$ to furnish S27 (516 mg, 87\%). Analytical data for S27: dark-yellow oil; ${ }^{1} \mathrm{H}-\mathrm{NMR}\left(400 \mathrm{MHz}, \mathrm{CDCl}_{3}\right) \delta 7.58(\mathrm{dd}, J=$ 8.0, $1.2 \mathrm{~Hz}, 1 \mathrm{H}), 7.41(\mathrm{dd}, J=8.0,1.2 \mathrm{~Hz}, 1 \mathrm{H}), 7.29(\mathrm{td}, J=8.0,1.2 \mathrm{~Hz}, 1 \mathrm{H}), 7.12(\mathrm{td}, J=8.0,1.2 \mathrm{~Hz}, 1 \mathrm{H})$, 4.47 (s, 2H), $3.32(\mathrm{~s}, 3 \mathrm{H}), 2.65(\mathrm{t}, J=7.6 \mathrm{~Hz}, 4 \mathrm{H}), 2.16-2.03(\mathrm{~m}, 1 \mathrm{H}), 1.70-1.60(\mathrm{~m}, 1 \mathrm{H}) ;{ }^{13} \mathrm{C}-\mathrm{NMR}(101$ $\left.\mathrm{MHz} \mathrm{CHCl}_{3}\right) \delta 140.7,134.6,129.2,126.8,123.1,92.7,83.5,55.9,34.2,15.1$, One Csp $p^{2}$ signal is missing due to overlapping.; IR (neat) $1013 \mathrm{~cm}^{-1}$; HRMS (DART) $\mathrm{m} / z$ : $\left[\mathrm{M}+\mathrm{NH}_{4}\right]^{+}$Calcd for $\mathrm{C}_{12} \mathrm{H}_{19} \mathrm{BrNO}_{2} 288.0599$; Found 288.0602.

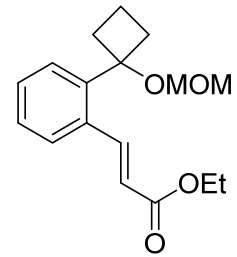

S28

To a solution of $\mathbf{S 2 7}(516 \mathrm{mg}, 1.91 \mathrm{mmol})$ in DMF $(2.00 \mathrm{~mL})$ was added $\mathrm{Pd}(\mathrm{OAc})_{2}(22.4 \mathrm{mg}$, $0.100 \mathrm{mmol}),(o-\mathrm{Tol})_{3} \mathrm{P}(60.9 \mathrm{mg}, 0.200 \mathrm{mmol}), \mathrm{NaHCO}_{3}(168 \mathrm{mg}, 2.00 \mathrm{mmol})$ and TBACl (556 mg, $2.00 \mathrm{mmol}$ ). After degassed at $-78^{\circ} \mathrm{C}$, the mixture was added ethyl acrylate $(870$ $\mu \mathrm{L}, 8.00 \mathrm{mmol}$ ). The reaction mixture was stirred at $100{ }^{\circ} \mathrm{C}$ overnight using an oil bath. After that, the reaction mixture was filtered through a pad of silica gel with a mixed solution of hexane $(40 \mathrm{~mL})$ and EtOAc $(10 \mathrm{~mL})$. The filtrate concentrated in vacuo. The obtained crude product was purified by silica gel column chromatography (Hexane/EtOAc $=20: 1)$ to give $\mathbf{S 2 8}(418 \mathrm{mg}, 72 \%)$. Analytical data for S28: pale-yellow oil; ${ }^{1} \mathrm{H}-\mathrm{NMR}\left(400 \mathrm{MHz}, \mathrm{CDCl}_{3}\right) \delta 8.26(\mathrm{~d}, J=16.0 \mathrm{~Hz}, 1 \mathrm{H}), 7.61(\mathrm{dd}, J=7.6,1.6$ 
$\mathrm{Hz}, 1 \mathrm{H}), 7.42(\mathrm{dd}, J=7.6,1.6 \mathrm{~Hz}, 1 \mathrm{H}), 7.35(\mathrm{td}, J=7.6,1.6 \mathrm{~Hz}, 1 \mathrm{H}), 7.31(\mathrm{td}, J=7.6,1.6 \mathrm{~Hz}, 1 \mathrm{H}), 6.28(\mathrm{~d}, J$ $=16.0 \mathrm{~Hz}, 1 \mathrm{H}), 4.42(\mathrm{~s}, 2 \mathrm{H}), 4.26(\mathrm{q}, J=7.2 \mathrm{~Hz}, 2 \mathrm{H}), 3.29(\mathrm{~s}, 3 \mathrm{H}), 2.65-2.53(\mathrm{~m}, 4 \mathrm{H}), 2.15-2.05(\mathrm{~m}, 1 \mathrm{H})$, $1.73-1.62(\mathrm{~m}, 1 \mathrm{H}), 1.33(\mathrm{t}, J=7.2 \mathrm{~Hz}, 3 \mathrm{H}) ;{ }^{13} \mathrm{C}-\mathrm{NMR}\left(101 \mathrm{MHz}, \mathrm{CDCl}_{3}\right) \delta 166.9,143.9,141.6,134.3,129.2$, 128.2, 127.7, 127.5, 119.0, 92.6, 82.2, 60.5, 55.9, 34.9, 15.1, 14.4; IR (neat) 1714, $1176 \mathrm{~cm}^{-1}$; HRMS (DART) $m / z:\left[\mathrm{M}+\mathrm{NH}_{4}\right]^{+}$Calcd for $\mathrm{C}_{17} \mathrm{H}_{26} \mathrm{NO}_{4} 308.1862$; Found 308.1890 .

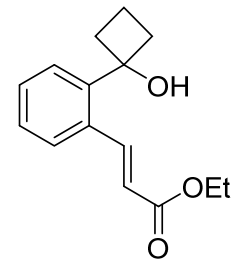

5

To a THF solution (5.0 mL) of $\mathbf{S 2 8}(311 \mathrm{mg}, 1.07 \mathrm{mmol})$ was added aq. $\mathrm{HCl}$ (5.0 mL, 3.00 $\mathrm{M})$. The resulting mixture was stirred at room temperature for $6 \mathrm{~h}$. The reaction was quenched with sat. aq. $\mathrm{NaHCO}_{3}$. The aqueous layer was extracted with $\mathrm{Et}_{2} \mathrm{O}$. The combined organic layer was washed with brine, dried over $\mathrm{MgSO}_{4}$, and concentrated in vacuo. The residue was purified by a silica gel column chromatography (Hexane/EtOAc $=10: 1)$ to furnish $\mathbf{5}(163 \mathrm{mg}$, 62\%). Analytical data for 5: pale-yellow oil; ${ }^{1} \mathrm{H}-\mathrm{NMR}\left(400 \mathrm{MHz}, \mathrm{CDCl}_{3}\right) \delta 8.24(\mathrm{~d}, J=16.0 \mathrm{~Hz}, 1 \mathrm{H}), 7.59$ $(\mathrm{dd}, J=7.6,1.6 \mathrm{~Hz}, 1 \mathrm{H}), 7.38(\mathrm{dd}, J=7.6,1.6 \mathrm{~Hz}, 1 \mathrm{H}), 7.34(\mathrm{td}, J=7.6,1.6 \mathrm{~Hz}, 1 \mathrm{H}), 7.29$ (td, $J=7.6,1.6$ $\mathrm{Hz}, 1 \mathrm{H}), 6.28(\mathrm{~d}, J=16.0 \mathrm{~Hz}, 1 \mathrm{H}), 4.24(\mathrm{q}, J=7.2 \mathrm{~Hz}, 2 \mathrm{H}), 2.70-2.63(\mathrm{~m}, 2 \mathrm{H}), 2.48(\mathrm{~s}, 1 \mathrm{H}), 2.47-2.41$ (m, 2H), 2.20-2.10 (m, 1H), 1.74-1.64 (m, 1H), $1.32(\mathrm{t}, J=7.2 \mathrm{~Hz}, 3 \mathrm{H}) ;{ }^{13} \mathrm{C}-\mathrm{NMR}\left(101 \mathrm{MHz}, \mathrm{CDCl}_{3}\right) \delta 167.0$, 144.2, 143.8, 133.9, 129.6, 128.1, 127.8, 125.7, 119.3, 78.1, 60.6, 36.9, 14.6, 14.4; IR (neat) 3446, $1698 \mathrm{~cm}^{-1}$; HRMS (DART) $m / z:\left[\mathrm{M}+\mathrm{NH}_{4}\right]^{+}$Calcd for $\mathrm{C}_{15} \mathrm{H}_{22} \mathrm{NO}_{3} 264.1600$; Found 264.1586.

\section{Preparation of the cyclopentanol 6}
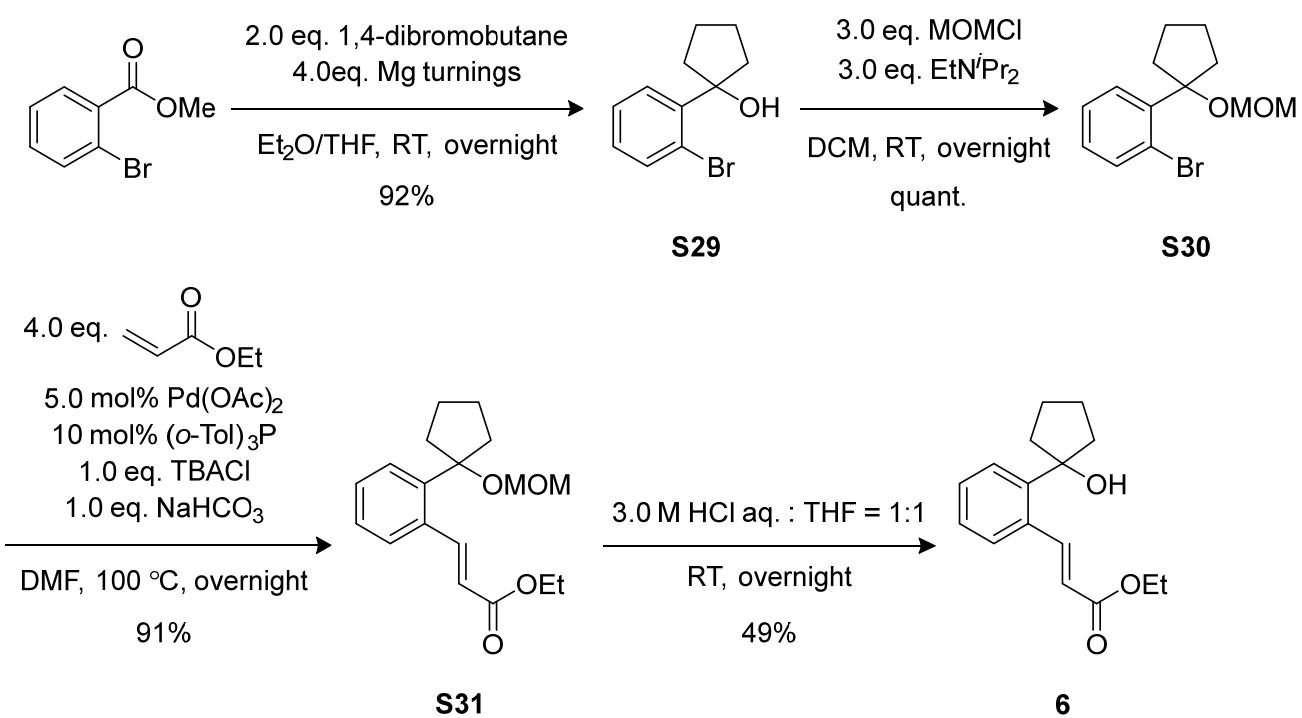

S31

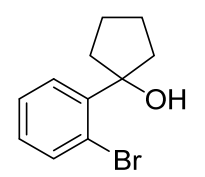

In a $100 \mathrm{~mL}$ two-necked flask containing $\mathrm{Mg}$ metal turnings (972 mg, $40.0 \mathrm{mmol})$ in dry $^{\mathrm{Et}} \mathrm{H}_{2} \mathrm{O}$ $(10 \mathrm{~mL})$, solution of 1,4-dibromobutane (4.32 $\mathrm{g}, 20.0 \mathrm{mmol})$ in anhydrous THF $(20 \mathrm{~mL}) \mathrm{was}$ added cautiously in a dropwise manner at $0{ }^{\circ} \mathrm{C}$. After addition, the reaction mixture was allowed

S29 to warm up to room temperature and stirred for $2 \mathrm{~h}$. The reaction mixture becomes turbid indicating generation of a Grignard reagent. To a solution of methyl 2-bromobenzoate (1.40 mL, $10.0 \mathrm{mmol})$ 
in THF (40 mL) was added the above generated solution of Grignard reagent slowly in a dropwise manner at $-78^{\circ} \mathrm{C}$. After addition, the reaction mixture was warmed up to room temperature and then stirred overnight. The suspension was quenched slowly with the addition of sat. $\mathrm{NH}_{4} \mathrm{Cl}$ solution, followed by extraction with EtOAc. The combined organic extracts were washed with water and brine, dried over anhydrous $\mathrm{MgSO}_{4}$, filtered and concentrated under reduced pressure. The residue was purified by a silica gel column chromatography (Hexane/EtOAc $=20: 1)$ to furnish $\mathbf{S 2 9}$ as a white solid (2.15 g, 89\%). Spectra are consistent with reported literature values. ${ }^{18}$ Analytical data for S29: white solid; ${ }^{1} \mathrm{H}-\mathrm{NMR}\left(400 \mathrm{MHz}, \mathrm{CDCl}_{3}\right) \delta 7.63$ $(\mathrm{dd}, J=7.6,1.2 \mathrm{~Hz}, 1 \mathrm{H}), 7.45(\mathrm{dd}, J=7.6,1.6 \mathrm{~Hz}, 1 \mathrm{H}), 7.25$ (td, $J=7.6,1.2 \mathrm{~Hz}, 1 \mathrm{H}), 7.11(\mathrm{td}, J=7.6,1.6$ $\mathrm{Hz}, 1 \mathrm{H}), 4.43$ (s, 2H), 3.36 (s, 3H), 2.64-2.59 (m, 2H), 2.08-2.00 (m, 2H), 1.96-1.91 (m, 2H), 1.76-1.67 (m, $2 \mathrm{H}) ;{ }^{13} \mathrm{C}-\mathrm{NMR}\left(101 \mathrm{MHz}, \mathrm{CHCl}_{3}\right) \delta 141.4,135.5,129.7,129.0,126.9,123.3,93.0,89.6,56.4,37.5,23.1$.

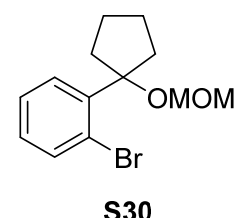

To a solution of $\mathbf{S 2 9}(493 \mathrm{mg}, 2.04 \mathrm{mmol})$ and $\mathrm{EtN}^{i} \operatorname{Pr}_{2}(1.20 \mathrm{~mL}, 6.93 \mathrm{mmol})$ in dry DCM $(10 \mathrm{~mL})$ was added $\mathrm{MOMCl}(530 \mu \mathrm{L}, 7.04 \mathrm{mmol})$ at $0{ }^{\circ} \mathrm{C}$, and the mixture was allowed to warm to rt. After being stirred at rt overnight, the reaction was quenched with water and extracted with EtOAc. The organic layer was washed with brine, dried over $\mathrm{MgSO}_{4}$, and concentrated in vacuo. The residue was purified by silica gel column chromatography $($ Hexane/EtOAc $=20: 1)$ to furnish S30 (576 mg, quant.). Analytical data for S30: dark-yellow oil; ${ }^{1} \mathrm{H}-\mathrm{NMR}\left(400 \mathrm{MHz}, \mathrm{CDCl}_{3}\right) \delta 7.63$ (dd, $J=7.6,1.2 \mathrm{~Hz}, 1 \mathrm{H}), 7.45$ (dd, $J=7.6,1.6 \mathrm{~Hz}, 1 \mathrm{H}), 7.25$ (td, $J=7.6,1.2 \mathrm{~Hz}, 1 \mathrm{H}), 7.11$ (td, $J=7.6,1.6$ $\mathrm{Hz}, 1 \mathrm{H}), 4.43$ (s, 2H), 3.36 (s, 3H), 2.64-2.59 (m, 2H), 2.08-2.00 (m, 2H), 1.96-1.91 (m, 2H), 1.76-1.67 (m, 2H); ${ }^{13} \mathrm{C}-\mathrm{NMR}\left(101 \mathrm{MHz}, \mathrm{CHCl}_{3}\right) \delta 141.4,135.5,129.7,129.0,126.9,123.3,93.0,89.6,56.4,37.5,23.1$; IR (neat) $1031 \mathrm{~cm}^{-1}$; HRMS (DART) $\mathrm{m} / z$ : $\left[\mathrm{M}+\mathrm{NH}_{4}\right]^{+}$Calcd for $\mathrm{C}_{13} \mathrm{H}_{21} \mathrm{BrNO}_{2} 302.0756$; Found 302.0762 .

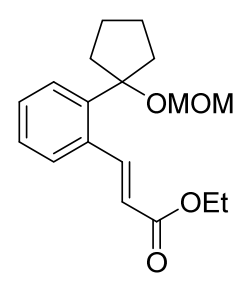

S31

To a solution of $\mathbf{S 3 0}(285 \mathrm{~g}, 1.00 \mathrm{mmol})$ in DMF $(2.00 \mathrm{~mL})$ was added $\mathrm{Pd}(\mathrm{OAc})_{2}(11.2 \mathrm{mg}$, $0.0500 \mathrm{mmol}),(o-\mathrm{Tol})_{3} \mathrm{P}(60.9 \mathrm{mg}, 0.100 \mathrm{mmol}), \mathrm{NaHCO}_{3}(84.0 \mathrm{mg}, 1.00 \mathrm{mmol})$ and TBACl (278 $\mathrm{mg}, 1.00 \mathrm{mmol}$ ). After degassed at $-78^{\circ} \mathrm{C}$, the mixture was added ethyl acrylate (870 $\mu \mathrm{L}, 8.00 \mathrm{mmol})$. The reaction mixture was stirred at $100^{\circ} \mathrm{C}$ overnight using an oil bath. After that, the reaction mixture was filtered through a pad of silica gel with a mixed solution of hexane $(40 \mathrm{~mL})$ and EtOAc $(10 \mathrm{~mL})$. The filtrate concentrated in vacuo. The obtained crude product was purified by silica gel column chromatography (Hexane/EtOAc $=20: 1)$ to give $\mathbf{S 3 1}(279 \mathrm{mg}, 91 \%)$. Analytical data for S31: pale-yellow solid (mp 50.5-51.7 $\left.{ }^{\circ} \mathrm{C}\right) ;{ }^{1} \mathrm{H}-\mathrm{NMR}\left(400 \mathrm{MHz}, \mathrm{CDCl}_{3}\right) \delta 8.59(\mathrm{~d}, J=$ $16.0 \mathrm{~Hz}, 1 \mathrm{H}), 7.57-7.55(\mathrm{~m}, 1 \mathrm{H}), 7.44-7.41(\mathrm{~m}, 1 \mathrm{H}), 7.33-7.29(\mathrm{~m}, 2 \mathrm{H}), 6.23(\mathrm{~d}, J=16.0 \mathrm{~Hz}, 1 \mathrm{H}), 4.40$ (s, 2H), 4.27 (q, $J=7.2 \mathrm{~Hz}, 2 \mathrm{H}), 3.32$ (s, 3H), 2.44-2.36 (m, 2H), 1.98-1.89 (m, 4H), 1.71-1.68 (m, 2H), 1.34 (t, $J=7.2 \mathrm{~Hz}, 3 \mathrm{H}) ;{ }^{13} \mathrm{C}-\mathrm{NMR}\left(101 \mathrm{MHz}, \mathrm{CDCl}_{3}\right) \delta 167.1,145.9,141.9,135.4,129.2,128.3,128.0,127.6,119.2$, 92.9, 89.2, 60.4, 56.2, 38.7, 23.2, 14.4; IR (KBr) 1711, $1156 \mathrm{~cm}^{-1}$; HRMS (DART) $\mathrm{m} / z$ : $\left[\mathrm{M}+\mathrm{NH}_{4}\right]^{+}$Calcd for $\mathrm{C}_{18} \mathrm{H}_{28} \mathrm{NO}_{4} 322.2018$; Found 322.2022. 
<smiles>CCOC(=O)/C=C/c1ccccc1C1(O)CCCC1</smiles>

To a THF solution $(2.0 \mathrm{~mL})$ of $\mathbf{S 3 1}(202 \mathrm{mg}, 0.660 \mathrm{mmol})$ was added aq. $\mathrm{HCl}(2.0 \mathrm{~mL}, 3.00$ M). The resulting mixture was stirred at room temperature overnight. The reaction was quenched with sat. aq. $\mathrm{NaHCO}_{3}$. The aqueous layer was extracted with $\mathrm{Et}_{2} \mathrm{O}$. The combined organic layer was washed with brine, dried over $\mathrm{MgSO}_{4}$, and concentrated in vacuo. The residue was purified by a silica gel column chromatography (Hexane/EtOAc $=10: 1)$ to furnish 6 (84.2 mg, 49\%). Analytical data for 6: pale-yellow solid (mp 73.2-73.8 $\left.{ }^{\circ} \mathrm{C}\right) ;{ }^{1} \mathrm{H}-\mathrm{NMR}(400$ $\left.\mathrm{MHz}, \mathrm{CDCl}_{3}\right) \delta 8.58(\mathrm{~d}, J=16.0 \mathrm{~Hz}, 1 \mathrm{H}), 7.54(\mathrm{dd}, J=7.6,1.6 \mathrm{~Hz}, 1 \mathrm{H}), 7.50(\mathrm{dd}, J=7.6,1.6 \mathrm{~Hz}, 1 \mathrm{H}), 7.32$ $(\mathrm{td}, J=7.6,1.6 \mathrm{~Hz}, 1 \mathrm{H}), 7.27(\mathrm{td}, J=7.6,1.6 \mathrm{~Hz}, 1 \mathrm{H}), 6.22(\mathrm{~d}, J=16.0 \mathrm{~Hz}, 1 \mathrm{H}), 4.26(\mathrm{q}, J=7.2 \mathrm{~Hz}, 2 \mathrm{H})$, 2.12-2.09 (m, 4H), 1.99-1.93 (m, 2H), 1.84-1.75 (m, 2H), 1.78 (s, 1H), 1.33 (t, $J=7.2 \mathrm{~Hz}, 3 \mathrm{H}) ;{ }^{13} \mathrm{C}-\mathrm{NMR}$ $\left(101 \mathrm{MHz}, \mathrm{CDCl}_{3}\right) \delta 167.1,145.8,145.0,134.8,129.3,128.5,127.7,125.6,119.3,83.7,60.5,41.3,23.9,14.4$; IR (KBr) 3454, $1695 \mathrm{~cm}^{-1}$; HRMS (DART) $\mathrm{m} / z$ : [M + NH4 $]^{+}$Calcd for $\mathrm{C}_{16} \mathrm{H}_{24} \mathrm{NO}_{3} 278.1756$; Found 278.1763.

\section{The ring expansion of 5 and 6}

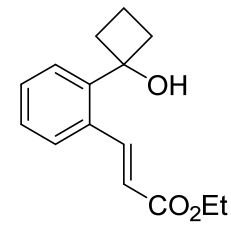

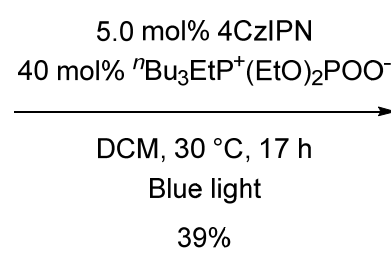

$39 \%$

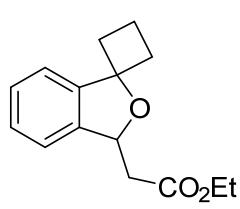

7

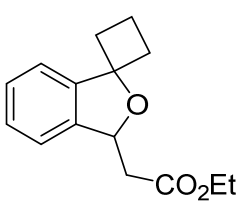

7

According to the representative procedure for the radical ring expansion of $\mathbf{1}$, pale-yellow oil was obtained in 39\% yield. Analytical data for 7: ${ }^{1} \mathrm{H}-\mathrm{NMR}\left(400 \mathrm{MHz}, \mathrm{CDCl}_{3}\right) \delta 7.46$ $(\mathrm{d}, J=7.6 \mathrm{~Hz}, 1 \mathrm{H}), 7.35$ (t, $J=7.6 \mathrm{~Hz}, 1 \mathrm{H}), 7.27$ (t, $J=7.6 \mathrm{~Hz}, 1 \mathrm{H}), 7.15$ (d, $J=7.6 \mathrm{~Hz}$, $1 \mathrm{H}), 5.60(\mathrm{t}, J=6.4 \mathrm{~Hz}, 1 \mathrm{H}), 4.20(\mathrm{q}, J=7.2 \mathrm{~Hz}, 2 \mathrm{H}), 2.74-2.59(\mathrm{~m}, 4 \mathrm{H}), 2.43-2.35(\mathrm{~m}$, 2H), 2.00-1.83 (m, 2H), $1.27(\mathrm{t}, J=7.2 \mathrm{~Hz}, 3 \mathrm{H}) ;{ }^{13} \mathrm{C}-\mathrm{NMR}\left(101 \mathrm{MHz}, \mathrm{CDCl}_{3}\right) \delta 170.9$, $145.9,140.5,128.5,127.6,121.0,120.0,87.5,79.0,60.7,42.8,38.8,37.8,14.3,13.0$; IR (neat) $1735 \mathrm{~cm}^{-1}$; HRMS (DART) $m / z:[\mathrm{M}+\mathrm{H}]^{+}$Calcd for $\mathrm{C}_{15} \mathrm{H}_{19} \mathrm{O}_{3}$ 247.1334; Found 247.1348.

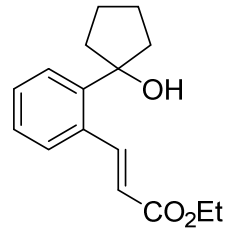

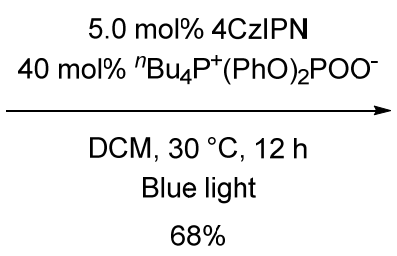

$68 \%$

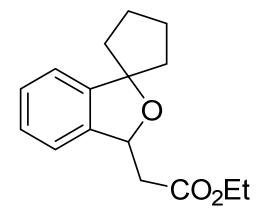

8

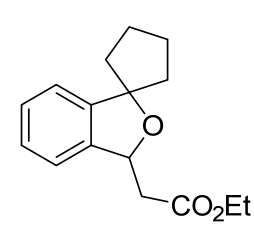

8

According to the representative procedure for the radical ring expansion of $\mathbf{1}$, pale-yellow oil was obtained in $68 \%$ yield. Analytical data for 8: ${ }^{1} \mathrm{H}-\mathrm{NMR}\left(400 \mathrm{MHz}, \mathrm{CDCl}_{3}\right) \delta 7.29$ $(\mathrm{td}, J=7.2,1.2 \mathrm{~Hz}, 1 \mathrm{H}), 7.25$ (td, $J=7.2,1.2 \mathrm{~Hz}, 1 \mathrm{H}), 7.16(\mathrm{~d}, J=7.2 \mathrm{~Hz}, 1 \mathrm{H}), 7.13(\mathrm{~d}, J=$ $7.2 \mathrm{~Hz}, 1 \mathrm{H}), 5.58(\mathrm{t}, J=6.8 \mathrm{~Hz}, 1 \mathrm{H}), 4.19(\mathrm{q}, J=7.2 \mathrm{~Hz}, 2 \mathrm{H}), 2.74(\mathrm{~d}, J=6.8 \mathrm{~Hz}, 2 \mathrm{H}), 2.10$ $1.88(\mathrm{~m}, 5 \mathrm{H}), 1.82-1.77(\mathrm{~m}, 3 \mathrm{H}), 1.26(\mathrm{t}, J=7.2 \mathrm{~Hz}, 3 \mathrm{H}) ;{ }^{13} \mathrm{C}-\mathrm{NMR}\left(101 \mathrm{MHz}, \mathrm{CDCl}_{3}\right) \delta$ 
171.0, 145.7, 141.3, 128.1, 127.4, 121.1, 120.8, 95.5, 78.1, 60.6, 42.8, 42.2, 41.1, 25.1, 25.0, 14.3; IR (neat) $1735 \mathrm{~cm}^{-1}$; HRMS (ESI) $\mathrm{m} / z$ : $[\mathrm{M}+\mathrm{Na}]^{+}$Calcd for $\mathrm{C}_{16} \mathrm{H}_{20} \mathrm{O}_{3} \mathrm{Na}$ 283.1310; Found 283.1296.

\section{Synthesis of S32 from 3z for X-ray crystallographic analysis}
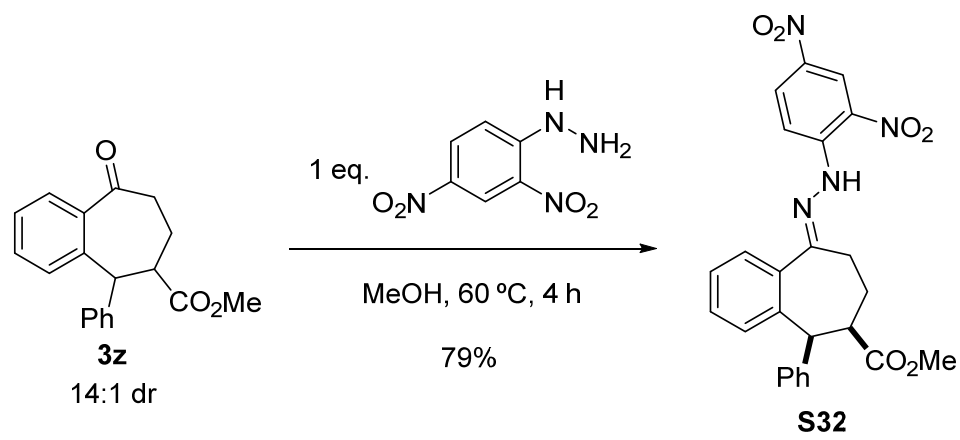

A solution of $\mathbf{3 z}(12.8 \mathrm{mg}, 0.043 \mathrm{mmol})$ and 2,4-dinitrophenylhydrazine $(8.4 \mathrm{mg}, 0.043 \mathrm{mmol})$ in $\mathrm{MeOH}(0.5 \mathrm{~mL})$ was stirred at $60^{\circ} \mathrm{C}$ for $4 \mathrm{~h}$ using an oil bath. The precipitate was collected by filtration and washed with water. The obtained solid was dried under reduced pressure to afford S32 (16.1 mg, 79\% yield) as an orange solid. The solid was recrystallized from $\mathrm{CH}_{2} \mathrm{Cl}_{2}$ and hexane. Analytical data for S32: $\mathrm{mp} 187.1-187.9{ }^{\circ} \mathrm{C} ;{ }^{1} \mathrm{H}-\mathrm{NMR}(400 \mathrm{MHz}$, $\left.\mathrm{CDCl}_{3}\right) \delta 11.46(\mathrm{~s}, 1 \mathrm{H}), 9.17(\mathrm{~d}, J=2.7 \mathrm{~Hz}, 1 \mathrm{H}), 8.36(\mathrm{dd}, J=9.6,2.7 \mathrm{~Hz}, 1 \mathrm{H}), 8.12(\mathrm{~d}, J=9.6 \mathrm{~Hz}, 1 \mathrm{H}), 7.72-$ $7.69(\mathrm{~m}, 1 \mathrm{H}), 7.42-7.37(\mathrm{~m}, 2 \mathrm{H}), 7.31-7.22(\mathrm{~m}, 4 \mathrm{H}), 7.09$ (d, $J=6.9 \mathrm{~Hz}, 2 \mathrm{H}), 4.95(\mathrm{~d}, J=3.2 \mathrm{~Hz}, 1 \mathrm{H}), 3.77$ (s, 3H), 3.33-3.27 (m, 1H), 2.85-2.76 (m, 2H), 2.40-2.32 (m, 1H), 1.97-1.87 (m, 1H); ${ }^{13} \mathrm{C}-\mathrm{NMR}(101 \mathrm{MHz}$, $\left.\mathrm{CDCl}_{3}\right) \delta 174.4,157.7,145.1,139.7,138.3,137.8,131.5,130.1,129.7,129.6,128.5,128.4,128.3,127.2$, 126.9, 123.5, 116.7, 76.7, 52.1, 49.0, 46.2, 26.3, 23.3; IR (neat) $1727 \mathrm{~cm}^{-1}$; HRMS (ESI) $m / z:[\mathrm{M}+\mathrm{Na}]^{+} \mathrm{Calcd}$ for $\mathrm{C}_{25} \mathrm{H}_{22} \mathrm{~N}_{4} \mathrm{O}_{6} \mathrm{Na}$ 497.1437; Found 497.1430. 


\section{Scale-Up Experiment (the data is shown in Table 2)}

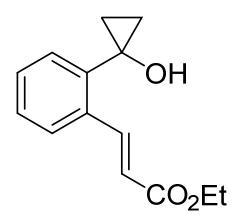

$1 \mathrm{a}$

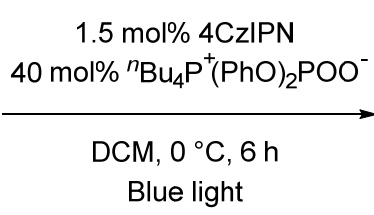

$72 \%$<smiles>CCOC(=O)CC1CCC(=O)c2ccccc21</smiles>

2a

4CzIPN (12.7 mg, $0.0161 \mathrm{mmol}),{ }^{n} \mathrm{Bu}_{4} \mathrm{P}^{+}\left(\mathrm{PhO}_{2} \mathrm{POO}^{-}(218 \mathrm{mg}, 0.428 \mathrm{mmol})\right.$, and $1 \mathrm{a}(249 \mathrm{mg}, 1.07 \mathrm{mmol})$ were dissolved in degassed dry DCM $(10 \mathrm{~mL})$ under Ar atmosphere. A LED lamp was put into the reaction mixture in the Schlenk tube, and the reaction mixture was stirred at $0{ }^{\circ} \mathrm{C}$ under irradiation. (Figure S2). After vigorous stirring at $0{ }^{\circ} \mathrm{C}$ for $6 \mathrm{~h}$, the resulting solution was concentrated in vacuo. The residue was then purified by a silica gel column chromatography (Hexane/EtOAc $=10: 1)$ to give $\mathbf{2 a}(179 \mathrm{mg}, 72 \%)$ as a pale-yellow oil.

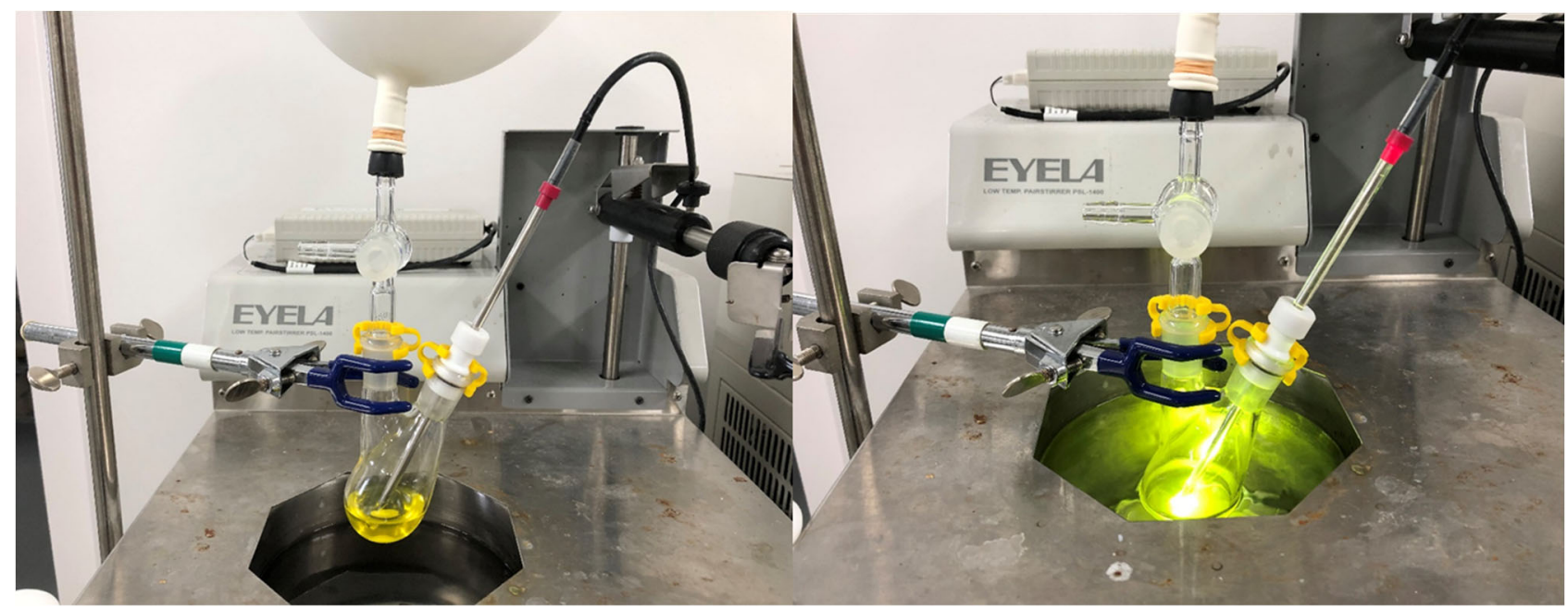

Figure S2. Scale-up experiment setup. 


\section{X-Ray Diffraction Analysis of S32}

Diffraction data were collected in $\theta$ ranges specified in Table S6 at $123 \mathrm{~K}$ on a Rigaku R-AXIS Rapid diffractometer with graphite monochromatized $\mathrm{Cu}$-Ka radiation $(\lambda=1.54187 \AA)$. The Lorenz polarization absorption correction was applied. The structure was solved by direct methods and refined by the full-matrix least-squares on $F^{2}$. All non-hydrogen atoms were refined with anisotropic displacement parameters. All hydrogen atoms were refined using the riding model. Final refinement details are compiled in Table S6. The supplementary crystallographic data for this paper (CCDC2084574) can also be obtained free of charge via www.ccdc.cam.ac.uk/conts/retrieving.html (or from the Cambridge Crystallographic Data Centre, 12, Union Road, Cambridge CB2 1EZ, UK; fax: +44 1223 336033; or deposit@ccdc.cam.ac.uk).

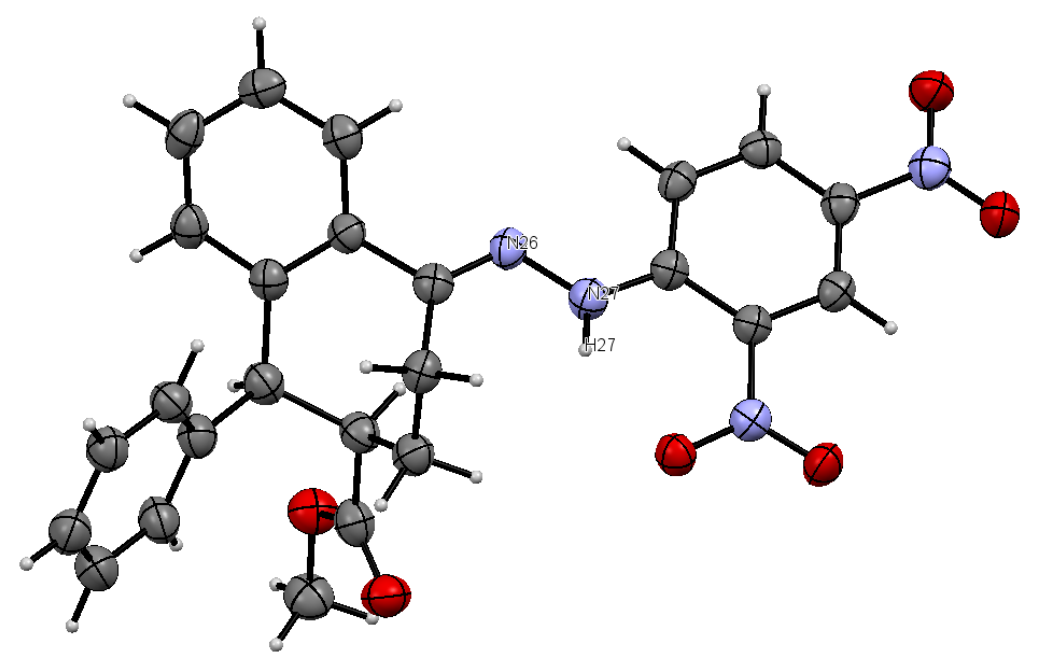

Figure S3. ORTEP plot of S32.

Table S6. Selected crystallographic data and collection parameters for $\mathbf{S 3 2}$.

\begin{tabular}{ll|ll}
\hline formula & $\mathrm{C}_{25} \mathrm{H}_{22} \mathrm{~N}_{4} \mathrm{O}_{6}$ & crystal size, mm & $0.2 \times 0.2 \times 0.1$ \\
$\mathrm{FW}$ & 474.47 & maximum $2 \theta$, deg & 136.3 \\
crystal system & monoclinic & reflections collected & 24007 \\
space group & $\mathrm{P} 21 / \mathrm{c}(\# 14)$ & independent reflections $[R(\mathrm{int})]$ & $3954[\mathrm{R}(\mathrm{int})=0.0656]$ \\
$a, \AA$ & $23.5028(16)$ & max. and min. transmission & $0.915 / 0.613$ \\
$b, \AA$ & $7.0069(5)$ & goodness-of-fit on $F^{2}$ & 1.020 \\
$c, \AA$ & $13.6822(9)$ & $R_{1}[I>2 \sigma(I)]$ & 0.0626 \\
volume, $\AA^{3}$ & $2166.4(3)$ & $R, w R_{2}($ all data $)$ & $0.1200,0.1683$ \\
$\beta,^{\circ}$ & $105.954(8)$ & Weighting scheme & $R_{1}=\Sigma\|\mathrm{Fo}|-| \mathrm{Fc}\| / \Sigma|\mathrm{Fo}|$ \\
$Z$ & 4 & & $w R_{2}=\left[\Sigma\left(\mathrm{w}\left(\mathrm{Fo}^{2}-\right.\right.\right.$ \\
& & & $\left.\left.\left.\mathrm{Fc}^{2}\right)^{2}\right) / \Sigma \mathrm{w}\left(\mathrm{Fo}^{2}\right)^{2}\right]^{1 / 2}$ \\
$D(\mathrm{calcd}), \mathrm{Mg} \mathrm{m}^{-3}$ & 1.455 & largest diff. peak and hole, e $\AA^{-3}$ & 0.28 and -0.25 \\
$\mu, \mathrm{cm}^{-1}$ & 8.841 & & \\
$F(000)$ & 992.00 & & \\
\hline
\end{tabular}




\section{DFT Calculations and Control Experiments}

The Gaussian 16 program package was used for all calculations. ${ }^{20}$ The geometries of the stationary points and transition states were fully optimized using the long-range corrected hybrid density functional of Chai and Head-Gordon $(\omega \mathrm{B} 97 \mathrm{X}-\mathrm{D})^{21}$ with the 6-31G(d) $)^{22}$ basis sets for all elements. The vibrational frequencies and the thermal correction to Gibbs free energy (TCGFE) including the zero-point energy were calculated at the same level of theory. The obtained structures were characterized by the number of imaginary frequencies (one or zero for the transition and ground states, respectively). The connectivity of each step was also confirmed using intrinsic reaction coordinate (IRC) ${ }^{23}$ calculation from the transition states, followed by optimization of the resultant geometries. Single-point energies for geometries obtained using the above method were calculated at the same level of theory using the $6-311++G(d, p)$ basis sets $^{24}$ for all elements. To examine the solvent effect, the above single-point energy calculations were performed using the $\mathrm{SMD}$ model ${ }^{25}$ with $\mathrm{CH}_{2} \mathrm{Cl}_{2}$ as the solvent. CYLview (Ver. 1.0b) ${ }^{26}$ was used for the visualization of the optimized structures.

Table S7. Summary of theoretical calculations.

\begin{tabular}{|c|c|c|c|}
\hline Model & TCGFE/au & SMD Energy/au & $\mathrm{IF} / \mathrm{cm}^{-1}$ \\
\hline Ia & 0.188841 & -728.767525 & \\
\hline TS $_{\text {Ia-IIa }}$ & 0.187971 & -728.767141 & $404.9162 \mathrm{i}$ \\
\hline IIa & 0.185546 & -728.781405 & \\
\hline IIa' & 0.185622 & -728.780014 & \\
\hline TS $_{\text {IIIa'-IIIa }}$ & 0.189125 & -728.776490 & $300.5464 \mathrm{i}$ \\
\hline IIIa & 0.193453 & -728.833847 & \\
\hline TS $_{\text {Ia-IVa }}$ & 0.190194 & -728.740673 & $601.5639 \mathrm{i}$ \\
\hline IVa & 0.191383 & -728.773497 & \\
\hline Ib & 0.218589 & -768.069888 & \\
\hline $\mathbf{T S}_{\mathrm{Ib}-\mathrm{IIb}}$ & 0.216516 & -768.066382 & $554.7307 \mathrm{i}$ \\
\hline IIb & 0.211792 & -768.094276 & \\
\hline TS $_{\text {IIb-IIIb }}$ & 0.216381 & -768.085778 & $399.3037 \mathrm{i}$ \\
\hline IIIb & 0.220411 & -768.135002 & \\
\hline $\mathbf{T S}_{\mathrm{Ib}-\mathrm{IVb}}$ & 0.218316 & -768.058769 & $380.8170 \mathrm{i}$ \\
\hline IVb & 0.219700 & -768.096583 & \\
\hline Ic & 0.247391 & -807.406864 & \\
\hline $\mathrm{TS}_{\text {Ic-IIc }}$ & 0.244996 & -807.395099 & $484.3396 \mathrm{i}$ \\
\hline IIc & 0.240495 & -807.409437 & \\
\hline TS $_{\text {Ic-IVe }}$ & 0.246969 & -807.401189 & $343.1856 \mathrm{i}$ \\
\hline IVe & 0.247538 & -807.440740 & \\
\hline
\end{tabular}


In the reaction of alkoxy radical Ia generated from cyclopropanol substrate 1, barrierless $\beta$-scission of Ia proceeds to generate the primary alkyl radical intermediate IIa. The cyclization of IIa occurs with a barrier of $\Delta \mathrm{G}^{\ddagger}=+5.4 \mathrm{kcal} \mathrm{mol}^{-1}$ to furnish IIIa. On the other hand, a barrier of the intramolecular Michael addition of Ia to generate IVa through $\mathbf{T S}_{\text {Ia-IVa }}$ is $\Delta \mathrm{G}^{\star}=+17.7 \mathrm{kcal} \mathrm{mol}^{-1}$, indicating that the cycloetherification of $\mathbf{1}$ is disfavored.

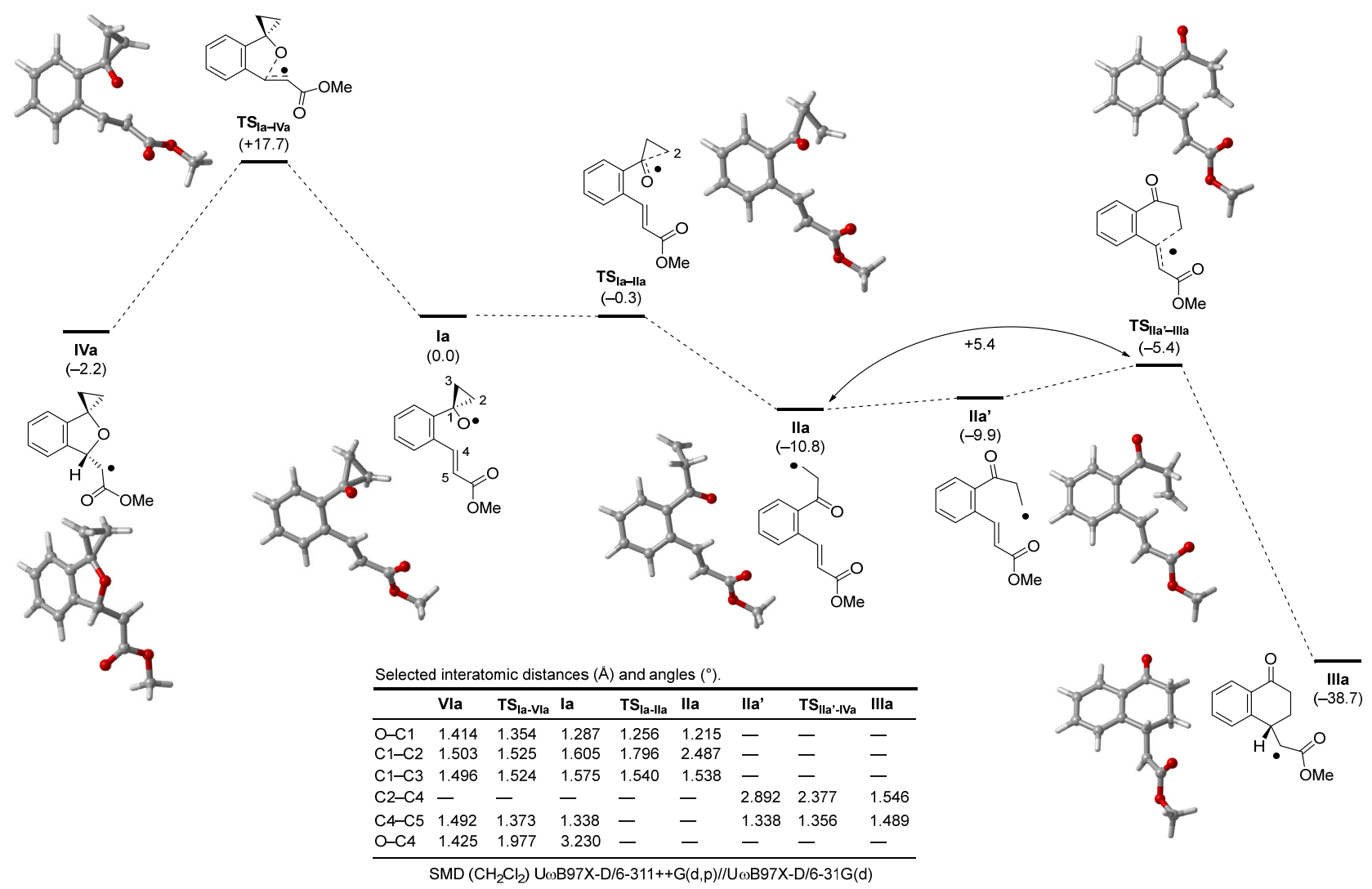

Figure S4. Energy surface for transformation of cyclopropoxy radical Ia into tetralone derivative IIIa and phthalan derivative IVa with relative Gibbs energies at $298 \mathrm{~K}$.

In the reaction of alkoxy radical $\mathbf{I b}$ generated from cyclobutanol substrate $\mathbf{5}, \beta$-scission of $\mathbf{I b}$ can proceed with a low activation energy of $\Delta \mathrm{G}^{\ddagger}=+0.9 \mathrm{kcal} \mathrm{mol}^{-1}$ to generate the primary alkyl radical intermediate IIb. On the other hand, a barrier of the intramolecular Michael addition of $\mathbf{I b}$ to generate $\mathbf{I V b}$ through $\mathbf{T S} \mathbf{S}_{\mathbf{I b}-\mathbf{I V b}}$ is $\Delta \mathrm{G}^{\ddagger}=+6.8 \mathrm{kcal} \mathrm{mol}^{-1}$, indicating that the cycloetherification of $\mathbf{5}$ is still disfavored. However, the reaction of 5 under the modified PCET conditions gave 7 and the desired cyclic ketone was not detected. 


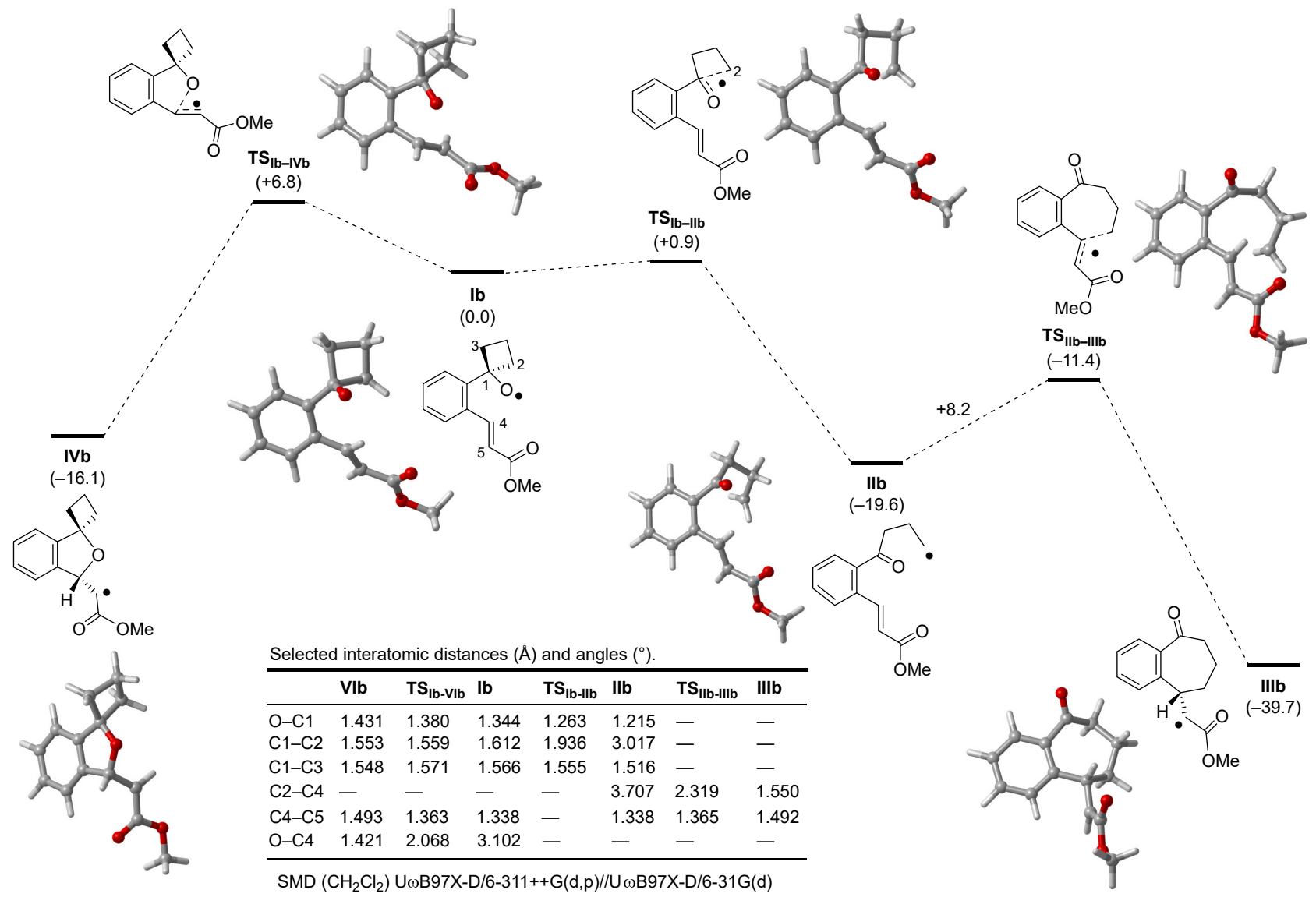

Figure S5. Energy surface for transformation of cyclobutoxy radical Ib into tetralone derivative IIIb and phthalan derivative $\mathbf{I V b}$ with relative Gibbs energies at $298 \mathrm{~K}$.

The reaction of 1-phenylcyclobutan-1-ol under the modified PCET conditions gave a trace amount of butyrophenone and 1-tetralone and $80 \%$ of starting material was recovered, which indicates that the PCET activation of the cyclobutanol is much less efficient than that of the cyclopropanol. However, the reaction of cyclobutanol substrate 5 in the presence of a base ${ }^{n} \mathrm{Bu}_{3} \mathrm{EtP}^{+}(\mathrm{EtO})_{2} \mathrm{POO}^{-}$also did not occur, which indicates that $\mathbf{7}$ was not formed via the ionic reaction process. Details for the transformation of $\mathbf{5}$ into $\mathbf{7}$ is not clear at the current stage.<smiles>OC1(c2ccccc2)CCC1</smiles>
$40 \mathrm{~mol}^{n}{ }^{n} \mathrm{Bu}_{3} \mathrm{EtP}^{+}(\mathrm{EtO})_{2} \mathrm{POO}^{-}$

$\mathrm{DCM}, 30^{\circ} \mathrm{C}, 17 \mathrm{~h}$

Blue light<smiles>CCOC(=CC=Cc1ccccc1C1(O)CCC1)CC</smiles>

5
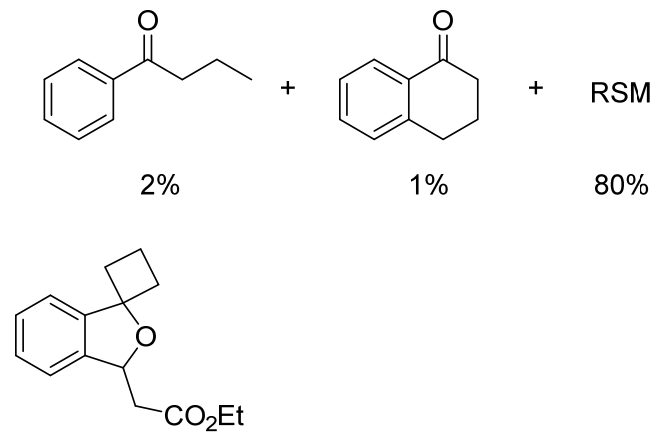

7 n.d.

Scheme S2. Control experiments with cyclobutanol substrates. 
In the reaction of alkoxy radical Ic generated from cyclopentanol substrate $\mathbf{6}$, a barrier of $\beta$-scission of Ic via $\mathbf{T S}_{\text {Ic-IIc }}$ to generate the primary alkyl radical intermediate IIc is $\Delta \mathrm{G}^{*}=+5.9 \mathrm{kcal} \mathrm{mol}^{-1}$. On the other hand, a barrier of the intramolecular Michael addition of Ic leading to IVc through $\mathbf{T S}_{\mathbf{I c}-\mathbf{I V c}}$ is $\Delta \mathrm{G}^{*}=+3.3 \mathrm{kcal} \mathrm{mol}^{-}$ ${ }^{1}$, indicating that the cycloetherification of $\mathbf{6}$ is favored unlike in the case of $\mathbf{1}$ and $\mathbf{5}$.

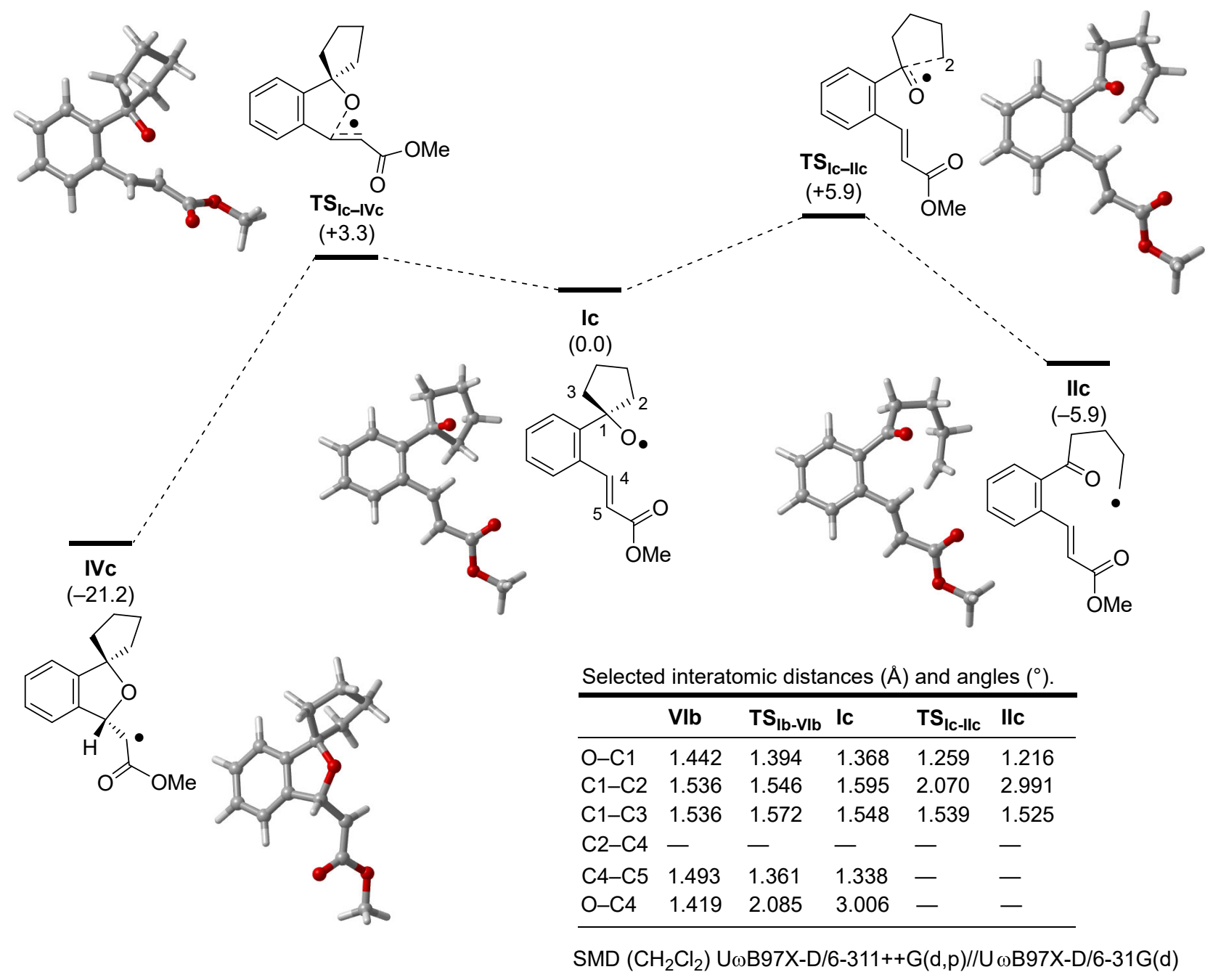

Figure S6. Energy surface for ring-opening and 5-exo cyclization of cyclopentoxy radical Ic with relative Gibbs energies at $298 \mathrm{~K}$. 


\section{Cartesian coordinates}

29

Ia

$\begin{array}{llll}\text { C } & 1.822200 & 3.025500 & -0.268800\end{array}$

$\begin{array}{llll}\mathrm{C} & 0.652100 & 2.302000 & -0.074100\end{array}$

$\begin{array}{llll}\mathrm{C} & 0.681400 & 0.915100 & 0.109000\end{array}$

$\begin{array}{llll}\text { C } & 1.925100 & 0.254200 & 0.100500\end{array}$

$\begin{array}{llll}\text { C } & 3.091400 & 0.990100 & -0.092600\end{array}$

$\begin{array}{llll}\mathrm{C} & 3.048000 & 2.368500 & -0.278100\end{array}$

$\mathrm{H} \quad 1.776500 \quad 4.102100 \quad-0.401700$

$\begin{array}{llll}\mathrm{H} & -0.300900 & 2.821400 & -0.036600\end{array}$

$\begin{array}{llll}\mathrm{H} & 4.049100 & 0.476700 & -0.087600\end{array}$

$\mathrm{H} \quad 3.968500 \quad 2.926600 \quad-0.419300$

$\begin{array}{llll}\text { C } & 1.999300 & -1.235800 & 0.337600\end{array}$

$\begin{array}{llll}\mathrm{C} & 1.483800 & -2.178500 & -0.855100\end{array}$

$\begin{array}{llll}\text { C } & 2.919200 & -2.094500 & -0.610100\end{array}$

$\mathrm{H} \quad 1.048300 \quad-1.624900 \quad-1.681200$

$\begin{array}{llll}\mathrm{H} & 0.928700 & -3.038900 & -0.500800\end{array}$

$\mathrm{H} \quad 3.530600 \quad-1.517900 \quad-1.297400$

$\mathrm{H} \quad 3.411600 \quad-2.913200 \quad-0.098200$

$\begin{array}{llll}\mathrm{O} & 1.782200 & -1.708700 & 1.514200\end{array}$

$\begin{array}{llll}\text { C } & -0.562800 & 0.152200 & 0.301800\end{array}$

$\mathrm{H} \quad-0.508200 \quad-0.751500 \quad 0.904100$

$\begin{array}{llll}\text { C } & -1.751100 & 0.477800 & -0.219800\end{array}$

$\mathrm{H} \quad-1.897500 \quad 1.344200 \quad-0.857700$

$\begin{array}{llll}\text { C } & -2.934600 & -0.367700 & 0.047300\end{array}$

$\begin{array}{llll}\mathrm{O} & -2.943400 & -1.394500 & 0.689500\end{array}$

$\begin{array}{llll}\text { O } & -4.033200 & 0.152700 & -0.531400\end{array}$

$\begin{array}{llll}\text { C } & -5.232200 & -0.591600 & -0.331500\end{array}$

$\begin{array}{llll}\mathrm{H} & -5.465300 & -0.667500 & 0.733600\end{array}$

$\mathrm{H} \quad-6.012800 \quad-0.039900 \quad-0.855000$

$\mathrm{H} \quad-5.133500 \quad-1.599300 \quad-0.743300$

29

TS $_{\text {Ia-IIa }}$

$\begin{array}{llll}\text { C } & 1.643400 & 3.257600 & -0.233200\end{array}$

$\begin{array}{llll}\text { C } & 0.479500 & 2.531400 & -0.011200\end{array}$ 


\begin{tabular}{lrrr}
$\mathrm{C}$ & 0.515100 & 1.148300 & 0.195500 \\
$\mathrm{C}$ & 1.763200 & 0.494100 & 0.181900 \\
$\mathrm{C}$ & 2.923300 & 1.231200 & -0.047000 \\
$\mathrm{C}$ & 2.871700 & 2.606300 & -0.252200 \\
$\mathrm{H}$ & 1.590200 & 4.331800 & -0.381600 \\
$\mathrm{H}$ & -0.475300 & 3.047200 & 0.027800 \\
$\mathrm{H}$ & 3.884600 & 0.724500 & -0.047300 \\
$\mathrm{H}$ & 3.787700 & 3.165600 & -0.415900 \\
$\mathrm{C}$ & 1.870700 & -0.986200 & 0.457800 \\
$\mathrm{C}$ & 1.351400 & -1.961300 & -0.958100 \\
$\mathrm{C}$ & 2.730200 & -1.844300 & -0.489200 \\
$\mathrm{H}$ & 0.979200 & -1.327000 & -1.754500 \\
$\mathrm{H}$ & 0.727900 & -2.780300 & -0.622700 \\
$\mathrm{H}$ & 3.418100 & -1.292900 & -1.126200 \\
$\mathrm{H}$ & 3.160300 & -2.707400 & 0.010400 \\
$\mathrm{O}$ & 1.456000 & -1.479700 & 1.535800 \\
$\mathrm{C}$ & -0.728200 & 0.384600 & 0.397600 \\
$\mathrm{H}$ & -0.669900 & -0.511200 & 1.010100 \\
$\mathrm{C}$ & -1.912000 & 0.706300 & -0.136600 \\
$\mathrm{H}$ & -2.052700 & 1.564900 & -0.786400 \\
$\mathrm{C}$ & -3.100500 & -0.132300 & 0.130300 \\
$\mathrm{O}$ & -3.119700 & -1.151600 & 0.783700 \\
$\mathrm{O}$ & -4.192300 & 0.386300 & -0.463900 \\
$\mathrm{C}$ & -5.395900 & -0.350700 & -0.265500 \\
$\mathrm{H}$ & -5.637700 & -0.414400 & 0.798600 \\
& -6.170100 & 0.198500 & -0.800900 \\
$\mathrm{H}$ & -5.298300 & -1.363200 & -0.665600 \\
\hline & & & \\
$\mathrm{H}$ & & & \\
$\mathrm{H}$ & & &
\end{tabular}

IIa

$\begin{array}{llll}\mathrm{C} & 1.593300 & 2.895000 & -0.394300 \\ \mathrm{C} & 0.434600 & 2.197900 & -0.076700 \\ \mathrm{C} & 0.414500 & 0.799500 & -0.021000 \\ \mathrm{C} & 1.616100 & 0.099500 & -0.270300 \\ \mathrm{C} & 2.778200 & 0.815600 & -0.571900 \\ \mathrm{C} & 2.772600 & 2.202200 & -0.646200 \\ \mathrm{H} & 1.576300 & 3.979900 & -0.433400 \\ \mathrm{H} & -0.474500 & 2.742800 & 0.158600\end{array}$




$\begin{array}{lrrr}\mathrm{H} & 3.698000 & 0.279900 & -0.781700 \\ \mathrm{H} & 3.683900 & 2.737000 & -0.894000 \\ \mathrm{C} & 1.641800 & -1.398000 & -0.257700 \\ \mathrm{C} & 3.643400 & -2.283600 & -1.438100 \\ \mathrm{C} & 2.992400 & -2.116900 & -0.106700 \\ \mathrm{H} & 4.615900 & -1.856800 & -1.655600 \\ \mathrm{H} & 3.118700 & -2.809400 & -2.228200 \\ \mathrm{H} & 3.645100 & -1.584300 & 0.591100 \\ \mathrm{H} & 2.745200 & -3.094000 & 0.327700 \\ \mathrm{O} & 0.627300 & -2.052700 & -0.389100 \\ \mathrm{C} & -0.843300 & 0.123200 & 0.346300 \\ \mathrm{H} & -0.796100 & -0.738300 & 1.005100 \\ \mathrm{C} & -2.049000 & 0.523800 & -0.070600 \\ \mathrm{H} & -2.184300 & 1.334800 & -0.779700 \\ \mathrm{C} & -3.264900 & -0.170300 & 0.403800 \\ \mathrm{O} & -3.298800 & -1.070300 & 1.213300 \\ \mathrm{O} & -4.364800 & 0.337000 & -0.185100 \\ \mathrm{C} & -5.593200 & -0.269800 & 0.205900 \\ \mathrm{H} & -5.755200 & -0.155400 & 1.280900 \\ \mathrm{H} & -6.370600 & 0.249200 & -0.354700 \\ \mathrm{H} & -5.591400 & -1.335200 & -0.038600\end{array}$

29

IIa'

$\begin{array}{lrrr}\mathrm{C} & 1.656700 & 3.111700 & -0.203300 \\ \mathrm{C} & 0.540700 & 2.299500 & -0.055300 \\ \mathrm{C} & 0.663400 & 0.921700 & 0.163900 \\ \mathrm{C} & 1.957300 & 0.366900 & 0.258500 \\ \mathrm{C} & 3.071700 & 1.201800 & 0.132900 \\ \mathrm{C} & 2.931100 & 2.561300 & -0.108400 \\ \mathrm{H} & 1.529700 & 4.176200 & -0.375600 \\ \mathrm{H} & -0.450200 & 2.742400 & -0.077200 \\ \mathrm{H} & 4.056100 & 0.756000 & 0.227100 \\ \mathrm{H} & 3.810600 & 3.189300 & -0.210400 \\ \mathrm{C} & 2.278900 & -1.091000 & 0.486200 \\ \mathrm{C} & 0.656900 & -1.908400 & -1.329500 \\ \mathrm{C} & 1.397500 & -2.209700 & -0.074900 \\ \mathrm{H} & 1.116400 & -1.322500 & -2.118500\end{array}$




$\begin{array}{lrrr}\mathrm{H} & -0.307900 & -2.362300 & -1.520400 \\ \mathrm{H} & 2.086900 & -3.058900 & -0.201100 \\ \mathrm{H} & 0.702300 & -2.524700 & 0.715400 \\ \mathrm{O} & 3.295500 & -1.383000 & 1.084300 \\ \mathrm{C} & -0.551400 & 0.112100 & 0.349400 \\ \mathrm{H} & -0.493400 & -0.750100 & 1.007300 \\ \mathrm{C} & -1.746700 & 0.360200 & -0.199600 \\ \mathrm{H} & -1.928100 & 1.182300 & -0.884300 \\ \mathrm{C} & -2.873900 & -0.551600 & 0.084500 \\ \mathrm{O} & -2.809000 & -1.566700 & 0.744900 \\ \mathrm{O} & -4.004100 & -0.119300 & -0.500200 \\ \mathrm{C} & -5.150000 & -0.941700 & -0.289500 \\ \mathrm{H} & -5.379800 & -1.015100 & 0.776500 \\ \mathrm{H} & -5.964800 & -0.453700 & -0.823500 \\ \mathrm{H} & -4.980100 & -1.946500 & -0.684400\end{array}$

29

TS $_{\text {IIa'-IIIa }}$

$\begin{array}{lrrr}\mathrm{C} & 1.825800 & 3.131800 & -0.212100 \\ \mathrm{C} & 0.741800 & 2.267700 & -0.123700 \\ \mathrm{C} & 0.917900 & 0.887600 & 0.032600 \\ \mathrm{C} & 2.234000 & 0.386500 & 0.120600 \\ \mathrm{C} & 3.314900 & 1.270400 & 0.049000 \\ \mathrm{C} & 3.121600 & 2.633100 & -0.124100 \\ \mathrm{H} & 1.655000 & 4.197000 & -0.335700 \\ \mathrm{H} & -0.265600 & 2.670700 & -0.145500 \\ \mathrm{H} & 4.313800 & 0.855200 & 0.130200 \\ \mathrm{H} & 3.974500 & 3.302000 & -0.181800 \\ \mathrm{C} & 2.599900 & -1.065300 & 0.269100 \\ \mathrm{C} & 0.570700 & -1.793500 & -1.109000 \\ \mathrm{C} & 1.619400 & -2.169000 & -0.120600 \\ \mathrm{H} & 0.855800 & -1.266200 & -2.014800 \\ \mathrm{H} & -0.345100 & -2.372800 & -1.145400 \\ \mathrm{H} & 2.247200 & -2.997800 & -0.480300 \\ \mathrm{H} & 1.155300 & -2.537500 & 0.805000 \\ \mathrm{O} & 3.701200 & -1.373600 & 0.681400 \\ \mathrm{C} & -0.279200 & 0.025500 & 0.162700 \\ \mathrm{H} & -0.271200 & -0.744300 & 0.929900\end{array}$




$\begin{array}{lrrr}\mathrm{C} & -1.485500 & 0.309900 & -0.388700 \\ \mathrm{H} & -1.629000 & 1.107500 & -1.109800 \\ \mathrm{C} & -2.631800 & -0.555700 & -0.087300 \\ \mathrm{O} & -2.592000 & -1.567500 & 0.585000 \\ \mathrm{O} & -3.758200 & -0.100100 & -0.667500 \\ \mathrm{C} & -4.922000 & -0.888300 & -0.433600 \\ \mathrm{H} & -5.143100 & -0.942500 & 0.635500 \\ \mathrm{H} & -5.730000 & -0.385900 & -0.965100 \\ \mathrm{H} & -4.784700 & -1.902800 & -0.816800\end{array}$

IIIa

$\begin{array}{lrrr}\mathrm{C} & 1.366700 & 3.018800 & 0.169300 \\ \mathrm{C} & 0.406100 & 2.014400 & 0.173700 \\ \mathrm{C} & 0.770600 & 0.669000 & 0.084800 \\ \mathrm{C} & 2.136500 & 0.350200 & 0.009000 \\ \mathrm{C} & 3.097700 & 1.365500 & -0.000400 \\ \mathrm{C} & 2.719800 & 2.696700 & 0.076600 \\ \mathrm{H} & 1.057600 & 4.057700 & 0.237300 \\ \mathrm{H} & -0.645900 & 2.278000 & 0.248200 \\ \mathrm{H} & 4.141800 & 1.076600 & -0.064100 \\ \mathrm{H} & 3.470700 & 3.480600 & 0.070900 \\ \mathrm{C} & 2.612200 & -1.066500 & -0.037600 \\ \mathrm{C} & 0.232700 & -1.719800 & -0.555300 \\ \mathrm{C} & 1.553000 & -2.142700 & 0.083300 \\ \mathrm{H} & 0.373500 & -1.556900 & -1.631400 \\ \mathrm{H} & -0.519800 & -2.504300 & -0.435000 \\ \mathrm{H} & 1.948500 & -3.062900 & -0.354400 \\ \mathrm{H} & 1.398100 & -2.332400 & 1.155500 \\ \mathrm{O} & 3.792400 & -1.339800 & -0.139500 \\ \mathrm{C} & -0.290700 & -0.418700 & 0.096100 \\ \mathrm{H} & -0.533100 & -0.667000 & 1.139700 \\ \mathrm{C} & -1.556300 & -0.003100 & -0.568600 \\ \mathrm{H} & -1.537300 & 0.615600 & -1.460600 \\ \mathrm{C} & -2.824000 & -0.539500 & -0.114200 \\ \mathrm{O} & -2.953500 & -1.304100 & 0.827500 \\ \mathrm{C} & -3.857800 & -0.099000 & -0.859800 \\ & -5.136300 & -0.593300 & -0.470900\end{array}$




$\begin{array}{llll}\mathrm{H} & -5.366700 & -0.302800 & 0.557500 \\ \mathrm{H} & -5.848500 & -0.144500 & -1.162900 \\ \mathrm{H} & -5.167300 & -1.683700 & -0.544000\end{array}$

29

TS $_{\text {Ia-IVa }}$

$\begin{array}{llll}\text { C } & 1.898900 & 3.368900 & 0.007700\end{array}$

$\begin{array}{llll}\text { C } & 0.702600 & 2.789500 & 0.427000\end{array}$

$\begin{array}{llll}\mathrm{C} & 0.561800 & 1.406400 & 0.395300\end{array}$

$\begin{array}{llll}\text { C } & 1.611800 & 0.604300 & -0.060500\end{array}$

$\begin{array}{llll}\text { C } & 2.799800 & 1.183000 & -0.489400\end{array}$

$\begin{array}{llll}\text { C } & 2.942300 & 2.568300 & -0.449400\end{array}$

$\begin{array}{llll}\mathrm{H} & 2.014500 & 4.447900 & 0.036500\end{array}$

$\begin{array}{llll}\mathrm{H} & -0.116700 & 3.412200 & 0.774300\end{array}$

$\begin{array}{llll}\mathrm{H} & 3.617500 & 0.562500 & -0.847100\end{array}$

$\begin{array}{llll}\mathrm{H} & 3.873100 & 3.023600 & -0.773900\end{array}$

$\begin{array}{llll}\text { C } & 1.233000 & -0.836600 & -0.067000\end{array}$

$\begin{array}{llll}\text { C } & 1.351400 & -1.653400 & -1.348800\end{array}$

$\begin{array}{llll}\text { C } & 2.236500 & -1.977000 & -0.188400\end{array}$

$\mathrm{H} \quad 1.787300 \quad-1.158900 \quad-2.212400$

$\mathrm{H} \quad 0.521500 \quad-2.322500 \quad-1.551300$

$\mathrm{H} \quad 3.286300 \quad-1.699000 \quad-0.222100$

$\begin{array}{llll}\mathrm{H} & 2.013300 & -2.861200 & 0.398100\end{array}$

$\begin{array}{llll}\text { O } & 0.202000 & -1.073400 & 0.777800\end{array}$

$\begin{array}{llll}\mathrm{C} & -0.685400 & 0.693500 & 0.793500\end{array}$

$\begin{array}{llll}\mathrm{H} & -0.940100 & 0.634200 & 1.848000\end{array}$

$\begin{array}{llll}\text { C } & -1.688900 & 0.443900 & -0.110100\end{array}$

$\begin{array}{llll}\mathrm{H} & -1.532000 & 0.563500 & -1.176700\end{array}$

$\begin{array}{llll}\text { C } & -2.975600 & -0.089600 & 0.354100\end{array}$

$\begin{array}{llll}\text { O } & -3.292800 & -0.255100 & 1.513400\end{array}$

$\begin{array}{llll}\mathrm{O} & -3.788300 & -0.368400 & -0.683800\end{array}$

$\begin{array}{llll}\text { C } & -5.066800 & -0.885100 & -0.326300\end{array}$

$\begin{array}{llll}\mathrm{H} & -5.613100 & -0.169700 & 0.294100\end{array}$

$\mathrm{H} \quad-5.591100 \quad-1.052600 \quad-1.267100$

$\mathrm{H} \quad-4.962800 \quad-1.822600 \quad 0.226100$ 


$\begin{array}{lrrr}\mathrm{C} & 1.153800 & 2.780500 & 0.057500 \\ \mathrm{C} & 0.245600 & 1.805800 & 0.468800 \\ \mathrm{C} & 0.604500 & 0.474700 & 0.316400 \\ \mathrm{C} & 1.826400 & 0.110900 & -0.231900 \\ \mathrm{C} & 2.731600 & 1.078600 & -0.646900 \\ \mathrm{C} & 2.383400 & 2.419700 & -0.496200 \\ \mathrm{H} & 0.902100 & 3.830700 & 0.169400 \\ \mathrm{H} & -0.715300 & 2.072000 & 0.897900 \\ \mathrm{H} & 3.691000 & 0.801200 & -1.076100 \\ \mathrm{H} & 3.078000 & 3.192700 & -0.811100 \\ \mathrm{C} & 1.904100 & -1.367600 & -0.260700 \\ \mathrm{C} & 2.460200 & -2.145300 & -1.420600 \\ \mathrm{C} & 3.164800 & -2.153000 & -0.080300 \\ \mathrm{H} & 2.902300 & -1.590200 & -2.243700 \\ \mathrm{H} & 1.921100 & -3.043900 & -1.703500 \\ \mathrm{H} & 4.097400 & -1.605600 & 0.027500 \\ \mathrm{H} & 3.088000 & -3.055600 & 0.517500 \\ \mathrm{O} & 0.737700 & -1.854100 & 0.372600 \\ \mathrm{C} & -0.166100 & -0.789400 & 0.653300 \\ \mathrm{H} & -0.429900 & -0.818500 & 1.718600 \\ \mathrm{C} & -1.403800 & -0.923900 & -0.169700 \\ \mathrm{H} & -1.366900 & -1.479400 & -1.099800 \\ \mathrm{C} & -2.618500 & -0.254300 & 0.237500 \\ \mathrm{O} & -2.718400 & 0.444500 & 1.235100 \\ \mathrm{O} & -3.640000 & -0.479800 & -0.612200 \\ \mathrm{H} & -4.864800 & 0.161000 & -0.267800 \\ & -4.738900 & 1.246700 & -0.242100 \\ \mathrm{H} & -5.573700 & -0.122200 & -1.045400 \\ \mathrm{H} & -5.215900 & -0.176200 & 0.711000\end{array}$

32

Ib

$\begin{array}{llll}\text { C } & 1.689400 & 3.225100 & -0.152400\end{array}$

$\begin{array}{llll}\text { C } & 0.520200 & 2.499300 & 0.042600\end{array}$

$\begin{array}{llll}\text { C } & 0.545000 & 1.106700 & 0.167000\end{array}$

$\begin{array}{llll}\text { C } & 1.779800 & 0.431900 & 0.087500\end{array}$

$\begin{array}{llll}\text { C } & 2.943500 & 1.172400 & -0.103600\end{array}$

$\begin{array}{llll}\text { C } & 2.907100 & 2.559300 & -0.221200\end{array}$ 


$\begin{array}{lrrr}\mathrm{H} & 1.648400 & 4.306700 & -0.237600 \\ \mathrm{H} & -0.429900 & 3.018500 & 0.129700 \\ \mathrm{H} & 3.900100 & 0.660700 & -0.155000 \\ \mathrm{H} & 3.829500 & 3.114900 & -0.360800 \\ \mathrm{C} & 1.860100 & -1.080800 & 0.224000 \\ \mathrm{C} & 0.988600 & -1.981900 & -0.789800 \\ \mathrm{C} & 3.093600 & -1.848300 & -0.360700 \\ \mathrm{C} & 2.164700 & -2.961900 & -0.878400 \\ \mathrm{H} & 0.840000 & -1.397300 & -1.702100 \\ \mathrm{H} & 0.029300 & -2.327700 & -0.402600 \\ \mathrm{H} & 3.540300 & -1.294100 & -1.190500 \\ \mathrm{H} & 3.860300 & -2.109900 & 0.371700 \\ \mathrm{H} & 2.389100 & -3.372500 & -1.865200 \\ \mathrm{H} & 2.061800 & -3.776300 & -0.159500 \\ \mathrm{O} & 1.506900 & -1.538600 & 1.437100 \\ \mathrm{C} & -0.706500 & 0.360900 & 0.380000 \\ \mathrm{H} & -0.665900 & -0.507400 & 1.032700 \\ \mathrm{C} & -1.883600 & 0.665900 & -0.177000 \\ \mathrm{H} & -2.013800 & 1.501600 & -0.858000 \\ \mathrm{C} & -3.069100 & -0.171400 & 0.105100 \\ \mathrm{O} & -3.076500 & -1.189000 & 0.763000 \\ \mathrm{O} & -4.167900 & 0.338300 & -0.481200 \\ \mathrm{C} & -5.366000 & -0.404700 & -0.271100 \\ \mathrm{H} & -5.600700 & -0.463700 & 0.794800 \\ \mathrm{H} & -6.146700 & 0.137100 & -0.804600 \\ & -5.265000 & -1.418800 & -0.666400\end{array}$

32

$\mathrm{TS}_{\mathrm{Ib}-\mathrm{IIb}}$

$\begin{array}{lrrr}\mathrm{C} & 1.692600 & 3.224900 & -0.216700 \\ \mathrm{C} & 0.529500 & 2.499300 & 0.013700 \\ \mathrm{C} & 0.562800 & 1.113700 & 0.198000 \\ \mathrm{C} & 1.804400 & 0.448000 & 0.143500 \\ \mathrm{C} & 2.962100 & 1.185600 & -0.096700 \\ \mathrm{C} & 2.914900 & 2.566000 & -0.269800 \\ \mathrm{H} & 1.642700 & 4.301700 & -0.345500 \\ \mathrm{H} & -0.423500 & 3.016100 & 0.079400 \\ \mathrm{H} & 3.922500 & 0.679500 & -0.132200\end{array}$




$\begin{array}{rrrr}\mathrm{H} & 3.831900 & 3.121900 & -0.440100 \\ \mathrm{C} & 1.917800 & -1.046300 & 0.390000 \\ \mathrm{C} & 0.906600 & -2.005300 & -0.953300 \\ \mathrm{C} & 3.044500 & -1.857800 & -0.310500 \\ \mathrm{C} & 2.113400 & -2.913700 & -0.925100 \\ \mathrm{H} & 0.839900 & -1.292700 & -1.774900 \\ \mathrm{H} & -0.051400 & -2.324000 & -0.551000 \\ \mathrm{H} & 3.532000 & -1.274900 & -1.096300 \\ \mathrm{H} & 3.792100 & -2.227100 & 0.396500 \\ \mathrm{H} & 2.425000 & -3.302000 & -1.899300 \\ \mathrm{H} & 1.953100 & -3.746300 & -0.238100 \\ \mathrm{O} & 1.437000 & -1.519500 & 1.457800 \\ \mathrm{C} & -0.684400 & 0.360800 & 0.414600 \\ \mathrm{H} & -0.631000 & -0.509100 & 1.062700 \\ \mathrm{C} & -1.858200 & 0.657600 & -0.154700 \\ \mathrm{H} & -1.990600 & 1.495100 & -0.833200 \\ \mathrm{C} & -3.039200 & -0.192500 & 0.106700 \\ \mathrm{O} & -3.042000 & -1.227900 & 0.736100 \\ \mathrm{O} & -4.141000 & 0.327100 & -0.466400 \\ \mathrm{C} & -5.335200 & -0.427200 & -0.276100 \\ \mathrm{H} & -5.570000 & -0.514800 & 0.787800 \\ \mathrm{H} & -6.118500 & 0.124100 & -0.796000 \\ \mathrm{H} & -5.228800 & -1.430300 & -0.697100\end{array}$

IIb

$\begin{array}{lrrr}\mathrm{C} & 1.662900 & 3.110800 & -0.431700 \\ \mathrm{C} & 0.515700 & 2.395900 & -0.111300 \\ \mathrm{C} & 0.579100 & 1.058500 & 0.295300 \\ \mathrm{C} & 1.844400 & 0.440200 & 0.373100 \\ \mathrm{C} & 2.988000 & 1.160300 & 0.024500 \\ \mathrm{C} & 2.906600 & 2.492600 & -0.363500 \\ \mathrm{H} & 1.584400 & 4.151900 & -0.729400 \\ \mathrm{H} & -0.450700 & 2.889000 & -0.150900 \\ \mathrm{H} & 3.961100 & 0.680600 & 0.080700 \\ \mathrm{H} & 3.809300 & 3.043700 & -0.607800 \\ \mathrm{C} & 2.003000 & -0.983200 & 0.840700 \\ \mathrm{C} & 1.152900 & -1.672300 & -1.970200\end{array}$




$\begin{array}{lrrr}\mathrm{C} & 2.792400 & -1.936700 & -0.035100 \\ \mathrm{C} & 1.810800 & -2.622000 & -1.028600 \\ \mathrm{H} & 1.745900 & -1.127600 & -2.698900 \\ \mathrm{H} & 0.107700 & -1.403400 & -1.863300 \\ \mathrm{H} & 3.574200 & -1.422600 & -0.602300 \\ \mathrm{H} & 3.250000 & -2.695700 & 0.605800 \\ \mathrm{H} & 2.392000 & -3.370500 & -1.585400 \\ \mathrm{H} & 1.061300 & -3.165300 & -0.444000 \\ \mathrm{O} & 1.460500 & -1.379200 & 1.853000 \\ \mathrm{C} & -0.658200 & 0.311300 & 0.584100 \\ \mathrm{H} & -0.608000 & -0.480900 & 1.325000 \\ \mathrm{C} & -1.827900 & 0.531800 & -0.027400 \\ \mathrm{H} & -1.955600 & 1.276700 & -0.807000 \\ \mathrm{C} & -3.018100 & -0.270500 & 0.332500 \\ \mathrm{O} & -3.054000 & -1.159300 & 1.153500 \\ \mathrm{O} & -4.087500 & 0.116600 & -0.388300 \\ \mathrm{C} & -5.290300 & -0.598200 & -0.116400 \\ \mathrm{H} & -5.580800 & -0.478900 & 0.930500 \\ \mathrm{H} & -6.044900 & -0.166900 & -0.773900 \\ \mathrm{H} & -5.161700 & -1.662900 & -0.327300\end{array}$

32

TS

$\begin{array}{lrrr}\mathrm{C} & 1.816300 & 3.200500 & -0.428800 \\ \mathrm{C} & 0.707900 & 2.364200 & -0.386900 \\ \mathrm{C} & 0.819300 & 1.033800 & 0.031200 \\ \mathrm{C} & 2.084900 & 0.562900 & 0.417500 \\ \mathrm{C} & 3.192000 & 1.408000 & 0.390400 \\ \mathrm{C} & 3.063900 & 2.724900 & -0.037200 \\ \mathrm{H} & 1.701900 & 4.229300 & -0.756300 \\ \mathrm{H} & -0.262700 & 2.761000 & -0.665800 \\ \mathrm{H} & 4.159400 & 1.030000 & 0.709100 \\ \mathrm{H} & 3.931600 & 3.377000 & -0.056400 \\ \mathrm{C} & 2.301800 & -0.862900 & 0.873700 \\ \mathrm{C} & 0.285100 & -1.435000 & -1.480200 \\ \mathrm{C} & 2.446800 & -1.928500 & -0.199900 \\ \mathrm{C} & 1.095400 & -2.442600 & -0.729000 \\ \mathrm{H} & 0.794500 & -0.762000 & -2.166600\end{array}$




$\begin{array}{lrrr}\mathrm{H} & -0.716600 & -1.734700 & -1.770100 \\ \mathrm{H} & 3.031200 & -1.521500 & -1.034800 \\ \mathrm{H} & 2.999600 & -2.759600 & 0.246900 \\ \mathrm{H} & 1.304100 & -3.298100 & -1.391500 \\ \mathrm{H} & 0.502800 & -2.849200 & 0.100200 \\ \mathrm{O} & 2.396300 & -1.128900 & 2.052300 \\ \mathrm{C} & -0.351200 & 0.132000 & 0.106800 \\ \mathrm{H} & -0.263500 & -0.718000 & 0.780000 \\ \mathrm{C} & -1.624300 & 0.459100 & -0.262200 \\ \mathrm{H} & -1.876600 & 1.360300 & -0.809200 \\ \mathrm{C} & -2.704000 & -0.492400 & 0.003900 \\ \mathrm{O} & -2.560800 & -1.610700 & 0.461200 \\ \mathrm{O} & -3.905700 & 0.012400 & -0.340900 \\ \mathrm{C} & -5.012600 & -0.855700 & -0.118800 \\ \mathrm{H} & -5.094800 & -1.114400 & 0.940100 \\ \mathrm{H} & -5.892500 & -0.301300 & -0.444900 \\ \mathrm{H} & -4.904600 & -1.776600 & -0.698100\end{array}$

IIIb

$\begin{array}{lrrr}\mathrm{C} & 1.030500 & 3.035500 & -0.098200 \\ \mathrm{C} & 0.225500 & 1.936800 & -0.382700 \\ \mathrm{C} & 0.663800 & 0.627300 & -0.173100 \\ \mathrm{C} & 1.952800 & 0.446200 & 0.360300 \\ \mathrm{C} & 2.740600 & 1.553900 & 0.682800 \\ \mathrm{C} & 2.295800 & 2.847000 & 0.442100 \\ \mathrm{H} & 0.656200 & 4.037600 & -0.285000 \\ \mathrm{H} & -0.778000 & 2.110600 & -0.759500 \\ \mathrm{H} & 3.716200 & 1.381100 & 1.126300 \\ \mathrm{H} & 2.926500 & 3.696300 & 0.685300 \\ \mathrm{C} & 2.592800 & -0.889500 & 0.634300 \\ \mathrm{C} & 0.247000 & -1.609300 & -1.408600 \\ \mathrm{C} & 2.627700 & -1.926900 & -0.473300 \\ \mathrm{C} & 1.279700 & -2.566400 & -0.817600 \\ \mathrm{H} & 0.655900 & -1.103200 & -2.293000 \\ \mathrm{H} & -0.605000 & -2.207200 & -1.753400 \\ \mathrm{H} & 3.032600 & -1.441500 & -1.372300 \\ \mathrm{H} & 3.333700 & -2.697400 & -0.151900\end{array}$




$\begin{array}{lrrc}\mathrm{H} & 1.465600 & -3.368800 & -1.540400 \\ \mathrm{H} & 0.862200 & -3.045200 & 0.077200 \\ \mathrm{O} & 3.173100 & -1.080300 & 1.684200 \\ \mathrm{C} & -0.288600 & -0.549800 & -0.411800 \\ \mathrm{H} & -0.447700 & -1.043700 & 0.555800 \\ \mathrm{C} & -1.633100 & -0.111600 & -0.886700 \\ \mathrm{H} & -1.756800 & 0.296300 & -1.886000 \\ \mathrm{C} & -2.815500 & -0.292000 & -0.067800 \\ \mathrm{O} & -2.827200 & -0.799200 & 1.040300 \\ \mathrm{O} & -3.924600 & 0.172300 & -0.682500 \\ \mathrm{C} & -5.129800 & 0.032000 & 0.064000 \\ \mathrm{H} & -5.064100 & 0.576000 & 1.010000 \\ \mathrm{H} & -5.915700 & 0.451700 & -0.563600 \\ \mathrm{H} & -5.332100 & -1.021100 & 0.277200\end{array}$

32

TS $_{\text {Ib-IVb }}$

$\begin{array}{lrrr}\mathrm{C} & 1.816300 & 3.200500 & -0.428800 \\ \mathrm{C} & 0.707900 & 2.364200 & -0.386900 \\ \mathrm{C} & 0.819300 & 1.033800 & 0.031200 \\ \mathrm{C} & 2.084900 & 0.562900 & 0.417500 \\ \mathrm{C} & 3.192000 & 1.408000 & 0.390400 \\ \mathrm{C} & 3.063900 & 2.724900 & -0.037200 \\ \mathrm{H} & 1.701900 & 4.229300 & -0.756300 \\ \mathrm{H} & -0.262700 & 2.761000 & -0.665800 \\ \mathrm{H} & 4.159400 & 1.030000 & 0.709100 \\ \mathrm{H} & 3.931600 & 3.377000 & -0.056400 \\ \mathrm{C} & 2.301800 & -0.862900 & 0.873700 \\ \mathrm{C} & 0.285100 & -1.435000 & -1.480200 \\ \mathrm{C} & 2.446800 & -1.928500 & -0.199900 \\ \mathrm{C} & 1.095400 & -2.442600 & -0.729000 \\ \mathrm{H} & 0.794500 & -0.762000 & -2.166600 \\ \mathrm{H} & -0.716600 & -1.734700 & -1.770100 \\ \mathrm{H} & 3.031200 & -1.521500 & -1.034800 \\ \mathrm{H} & 2.999600 & -2.759600 & 0.246900 \\ \mathrm{H} & 1.304100 & -3.298100 & -1.391500 \\ \mathrm{H} & 0.502800 & -2.849200 & 0.100200 \\ \mathrm{O} & 2.396300 & -1.128900 & 2.052300\end{array}$




$\begin{array}{lrrr}\mathrm{C} & -0.351200 & 0.132000 & 0.106800 \\ \mathrm{H} & -0.263500 & -0.718000 & 0.780000 \\ \mathrm{C} & -1.624300 & 0.459100 & -0.262200 \\ \mathrm{H} & -1.876600 & 1.360300 & -0.809200 \\ \mathrm{C} & -2.704000 & -0.492400 & 0.003900 \\ \mathrm{O} & -2.560800 & -1.610700 & 0.461200 \\ \mathrm{O} & -3.905700 & 0.012400 & -0.340900 \\ \mathrm{C} & -5.012600 & -0.855700 & -0.118800 \\ \mathrm{H} & -5.094800 & -1.114400 & 0.940100 \\ \mathrm{H} & -5.892500 & -0.301300 & -0.444900 \\ \mathrm{H} & -4.904600 & -1.776600 & -0.698100\end{array}$

IVb

$\begin{array}{lrrr}\mathrm{C} & 1.030500 & 3.035500 & -0.098200 \\ \mathrm{C} & 0.225500 & 1.936800 & -0.382700 \\ \mathrm{C} & 0.663800 & 0.627300 & -0.173100 \\ \mathrm{C} & 1.952800 & 0.446200 & 0.360300 \\ \mathrm{C} & 2.740600 & 1.553900 & 0.682800 \\ \mathrm{C} & 2.295800 & 2.847000 & 0.442100 \\ \mathrm{H} & 0.656200 & 4.037600 & -0.285000 \\ \mathrm{H} & -0.778000 & 2.110600 & -0.759500 \\ \mathrm{H} & 3.716200 & 1.381100 & 1.126300 \\ \mathrm{H} & 2.926500 & 3.696300 & 0.685300 \\ \mathrm{C} & 2.592800 & -0.889500 & 0.634300 \\ \mathrm{C} & 0.247000 & -1.609300 & -1.408600 \\ \mathrm{C} & 2.627700 & -1.926900 & -0.473300 \\ \mathrm{C} & 1.279700 & -2.566400 & -0.817600 \\ \mathrm{H} & 0.655900 & -1.103200 & -2.293000 \\ \mathrm{H} & -0.605000 & -2.207200 & -1.753400 \\ \mathrm{H} & 3.032600 & -1.441500 & -1.372300 \\ \mathrm{H} & 3.333700 & -2.697400 & -0.151900 \\ \mathrm{H} & 1.465600 & -3.368800 & -1.540400 \\ \mathrm{H} & 0.862200 & -3.045200 & 0.077200 \\ \mathrm{O} & 3.173100 & -1.080300 & 1.684200 \\ \mathrm{C} & -0.288600 & -0.549800 & -0.411800 \\ \mathrm{H} & -0.447700 & -1.043700 & 0.555800 \\ \mathrm{C} & -1.633100 & -0.111600 & -0.886700\end{array}$




$\begin{array}{lrrr}\mathrm{H} & -1.756800 & 0.296300 & -1.886000 \\ \mathrm{C} & -2.815500 & -0.292000 & -0.067800 \\ \mathrm{O} & -2.827200 & -0.799200 & 1.040300 \\ \mathrm{O} & -3.924600 & 0.172300 & -0.682500 \\ \mathrm{C} & -5.129800 & 0.032000 & 0.064000 \\ \mathrm{H} & -5.064100 & 0.576000 & 1.010000 \\ \mathrm{H} & -5.915700 & 0.451700 & -0.563600 \\ \mathrm{H} & -5.332100 & -1.021100 & 0.277200\end{array}$

35

Ic

$\begin{array}{llll}\text { C } & 1.270200 & 3.320500 & -1.117500\end{array}$

$\begin{array}{llll}\text { C } & 0.211900 & 2.617700 & -0.555000\end{array}$

$\begin{array}{llll}\mathrm{C} & 0.352400 & 1.285700 & -0.152300\end{array}$

$\begin{array}{llll}\text { C } & 1.597100 & 0.639400 & -0.322500\end{array}$

$\begin{array}{llll}\text { C } & 2.650100 & 1.364300 & -0.878400\end{array}$

$\begin{array}{llll}\mathrm{C} & 2.496500 & 2.690100 & -1.276000\end{array}$

$\mathrm{H} \quad 1.138400 \quad 4.356200 \quad-1.415300$

$\begin{array}{llll}\mathrm{H} & -0.740700 & 3.114000 & -0.394500\end{array}$

$\begin{array}{llll}\mathrm{H} & 3.620000 & 0.896100 & -1.004300\end{array}$

$\begin{array}{llll}\mathrm{H} & 3.340100 & 3.225800 & -1.700800\end{array}$

$\begin{array}{llll}\mathrm{C} & 1.803500 & -0.820500 & 0.100800\end{array}$

$\begin{array}{llll}\text { C } & 0.778400 & -1.832500 & -0.583400\end{array}$

$\begin{array}{llll}\text { C } & 3.168600 & -1.455700 & -0.258100\end{array}$

$\mathrm{H} \quad 0.411800 \quad-1.358000 \quad-1.497900$

$\mathrm{H} \quad-0.082300 \quad-1.989900 \quad 0.070200$

$\mathrm{H} \quad 3.427500 \quad-1.216300 \quad-1.296300$

$\begin{array}{llll}\mathrm{H} & 3.965900 & -1.084800 & 0.393000\end{array}$

$\begin{array}{llll}\mathrm{O} & 1.548800 & -0.991900 & 1.434100\end{array}$

$\begin{array}{llll}\text { C } & -0.804100 & 0.596200 & 0.446900\end{array}$

$\mathrm{H} \quad-0.611200 \quad-0.075500 \quad 1.278700$

$\begin{array}{llll}\text { C } & -2.068400 & 0.737300 & 0.033700\end{array}$

$\begin{array}{llll}\mathrm{H} & -2.346100 & 1.367000 & -0.806300\end{array}$

$\begin{array}{llll}\text { C } & -3.150900 & -0.009000 & 0.709300\end{array}$

$\begin{array}{llll}\text { O } & -3.005100 & -0.819000 & 1.598600\end{array}$

$\begin{array}{llll}\text { O } & -4.352100 & 0.318100 & 0.197100\end{array}$

$\begin{array}{llll}\text { C } & -5.463000 & -0.356800 & 0.781300\end{array}$

$\mathrm{H} \quad-5.530900 \quad-0.134500 \quad 1.849300$ 


$\begin{array}{lrrr}\mathrm{H} & -6.343200 & 0.015200 & 0.257200 \\ \mathrm{H} & -5.368700 & -1.438100 & 0.651900 \\ \mathrm{C} & 1.564600 & -3.131800 & -0.849800 \\ \mathrm{H} & 1.733100 & -3.246400 & -1.926300 \\ \mathrm{H} & 1.019400 & -4.015900 & -0.509100 \\ \mathrm{C} & 2.908400 & -2.953300 & -0.129900 \\ \mathrm{H} & 2.810200 & -3.217600 & 0.927100 \\ \mathrm{H} & 3.708500 & -3.561400 & -0.561300\end{array}$

TS $_{\text {Ic-IIc }}$

$\begin{array}{lrrr}\mathrm{C} & 1.302900 & 3.289600 & -2.178100 \\ \mathrm{C} & 0.257200 & 2.605300 & -1.570400 \\ \mathrm{C} & 0.423000 & 1.308600 & -1.073500 \\ \mathrm{C} & 1.684900 & 0.684800 & -1.194700 \\ \mathrm{C} & 2.723000 & 1.384500 & -1.811100 \\ \mathrm{C} & 2.542900 & 2.676200 & -2.297400 \\ \mathrm{H} & 1.149700 & 4.299700 & -2.545600 \\ \mathrm{H} & -0.706100 & 3.092300 & -1.451200 \\ \mathrm{H} & 3.702500 & 0.926400 & -1.899400 \\ \mathrm{H} & 3.374200 & 3.199800 & -2.759500 \\ \mathrm{C} & 1.961600 & -0.697500 & -0.619400 \\ \mathrm{C} & 0.681600 & -1.934000 & -1.677000 \\ \mathrm{C} & 3.202800 & -1.467100 & -1.103500 \\ \mathrm{H} & 0.393500 & -1.277600 & -2.495300 \\ \mathrm{H} & -0.101500 & -2.086300 & -0.940100 \\ \mathrm{H} & 3.473200 & -1.182400 & -2.126200 \\ \mathrm{H} & 4.043700 & -1.224200 & -0.444400 \\ \mathrm{O} & 1.549700 & -0.958200 & 0.541000 \\ \mathrm{C} & -0.724400 & 0.614300 & -0.461800 \\ \mathrm{H} & -0.515400 & -0.057300 & 0.365300 \\ \mathrm{C} & -1.988800 & 0.737200 & -0.882100 \\ \mathrm{H} & -2.273900 & 1.362700 & -1.722900 \\ \mathrm{C} & -3.065700 & -0.024800 & -0.215000 \\ \mathrm{O} & -2.915800 & -0.845300 & 0.663600 \\ \mathrm{O} & -4.269300 & 0.301000 & -0.724100 \\ \mathrm{C} & -5.375800 & -0.387800 & -0.148200 \\ \mathrm{H} & -5.444800 & -0.179100 & 0.922500\end{array}$




$\begin{array}{lrrr}\mathrm{H} & -6.258500 & -0.014900 & -0.667400 \\ \mathrm{H} & -5.275000 & -1.466900 & -0.290500 \\ \mathrm{C} & 1.606300 & -3.086100 & -1.967500 \\ \mathrm{H} & 1.920300 & -3.056600 & -3.017100 \\ \mathrm{H} & 1.099900 & -4.042600 & -1.796700 \\ \mathrm{C} & 2.845800 & -2.947600 & -1.065700 \\ \mathrm{H} & 2.594000 & -3.226700 & -0.038400 \\ \mathrm{H} & 3.668100 & -3.583900 & -1.406400\end{array}$

IIc

$\begin{array}{llll}\text { C } & 1.306600 & 3.338900 & -1.277800\end{array}$

$\begin{array}{llll}\text { C } & 0.266700 & 2.645400 & -0.671700\end{array}$

$\begin{array}{llll}\text { C } & 0.471600 & 1.396500 & -0.074500\end{array}$

$\begin{array}{llll}\text { C } & 1.770000 & 0.844400 & -0.100400\end{array}$

$\begin{array}{llll}\mathrm{C} & 2.801100 & 1.539400 & -0.735800\end{array}$

$\begin{array}{llll}\text { C } & 2.581600 & 2.785000 & -1.312000\end{array}$

$\begin{array}{llll}\mathrm{H} & 1.120500 & 4.313100 & -1.719500\end{array}$

$\begin{array}{llll}\mathrm{H} & -0.723400 & 3.089600 & -0.636400\end{array}$

$\begin{array}{llll}\mathrm{H} & 3.798400 & 1.109200 & -0.762000\end{array}$

$\begin{array}{llll}\mathrm{H} & 3.401700 & 3.319300 & -1.781200\end{array}$

$\begin{array}{llll}\text { C } & 2.100000 & -0.464800 & 0.565400\end{array}$

$\begin{array}{llll}\text { C } & 0.155000 & -2.257800 & -0.829600\end{array}$

$\begin{array}{llll}\text { C } & 3.045800 & -1.426100 & -0.147100\end{array}$

$\mathrm{H} \quad-0.325700 \quad-1.685700 \quad-1.615900$

$\mathrm{H} \quad-0.344100 \quad-2.304300 \quad 0.133800$

$\mathrm{H} \quad 3.220700 \quad-1.113100 \quad-1.181700$

$\begin{array}{llll}\mathrm{H} & 4.007500 & -1.375800 & 0.380500\end{array}$

$\begin{array}{llll}\mathrm{O} & 1.665000 & -0.740800 & 1.666800\end{array}$

$\begin{array}{llll}\text { C } & -0.660700 & 0.661000 & 0.515100\end{array}$

$\mathrm{H} \quad-0.445800 \quad-0.007900 \quad 1.341700$

$\begin{array}{llll}\text { C } & -1.921800 & 0.734100 & 0.074400\end{array}$

$\mathrm{H} \quad-2.223800 \quad 1.350100 \quad-0.767200$

$\begin{array}{llll}\text { C } & -2.971100 & -0.086800 & 0.715900\end{array}$

$\begin{array}{llll}\text { O } & -2.796800 & -0.897200 & 1.599300\end{array}$

$\begin{array}{llll}\text { O } & -4.177300 & 0.171300 & 0.176600\end{array}$

$\begin{array}{llll}\text { C } & -5.257100 & -0.582500 & 0.721800\end{array}$

$\mathrm{H} \quad-5.366100 \quad-0.381500 \quad 1.790600$ 


$\begin{array}{lrrr}\mathrm{H} & -6.145700 & -0.259600 & 0.179800 \\ \mathrm{H} & -5.089500 & -1.653200 & 0.579900 \\ \mathrm{C} & 1.358300 & -3.095800 & -1.097700 \\ \mathrm{H} & 1.718300 & -2.920100 & -2.119200 \\ \mathrm{H} & 1.088200 & -4.163600 & -1.046700 \\ \mathrm{C} & 2.517900 & -2.865100 & -0.109900 \\ \mathrm{H} & 2.193100 & -3.089300 & 0.911600 \\ \mathrm{H} & 3.334400 & -3.554600 & -0.352600\end{array}$

TS Ic-IVe

$\begin{array}{lrrr}\mathrm{C} & 1.619700 & 3.429100 & -1.053500 \\ \mathrm{C} & 0.556400 & 2.940700 & -0.300500 \\ \mathrm{C} & 0.476200 & 1.579000 & -0.011800 \\ \mathrm{C} & 1.464800 & 0.706900 & -0.471000 \\ \mathrm{C} & 2.518000 & 1.198300 & -1.236600 \\ \mathrm{C} & 2.598300 & 2.557100 & -1.524500 \\ \mathrm{H} & 1.681800 & 4.489900 & -1.276200 \\ \mathrm{H} & -0.215700 & 3.615200 & 0.058500 \\ \mathrm{H} & 3.281900 & 0.518400 & -1.606800 \\ \mathrm{H} & 3.426700 & 2.936800 & -2.115100 \\ \mathrm{C} & 1.259200 & -0.754200 & -0.121500 \\ \mathrm{C} & 0.829200 & -1.618100 & -1.329900 \\ \mathrm{C} & 2.529500 & -1.491000 & 0.440200 \\ \mathrm{H} & 1.390900 & -1.313300 & -2.222600 \\ \mathrm{H} & -0.237000 & -1.487500 & -1.534700 \\ \mathrm{H} & 3.420900 & -0.869400 & 0.320400 \\ \mathrm{H} & 2.383700 & -1.641700 & 1.513400 \\ \mathrm{O} & 0.335100 & -0.830700 & 0.919000 \\ \mathrm{C} & -0.654200 & 0.996900 & 0.755900 \\ \mathrm{H} & -0.688900 & 1.135300 & 1.833200 \\ \mathrm{C} & -1.767800 & 0.511600 & 0.142700 \\ \mathrm{H} & -1.826400 & 0.414300 & -0.936300 \\ \mathrm{C} & -2.905200 & 0.034100 & 0.948100 \\ \mathrm{O} & -2.998500 & 0.119200 & 2.153400 \\ \mathrm{O} & -3.853000 & -0.514000 & 0.165600 \\ \mathrm{C} & -4.995300 & -1.010100 & 0.858400 \\ \mathrm{H} & -5.493000 & -0.206100 & 1.406800\end{array}$




$\begin{array}{lrrr}\mathrm{H} & -5.654500 & -1.413200 & 0.089800 \\ \mathrm{H} & -4.706500 & -1.792800 & 1.564600 \\ \mathrm{C} & 1.223800 & -3.034600 & -0.917700 \\ \mathrm{H} & 1.211300 & -3.748700 & -1.746300 \\ \mathrm{H} & 0.532600 & -3.393500 & -0.146800 \\ \mathrm{C} & 2.621400 & -2.828800 & -0.319600 \\ \mathrm{H} & 2.937900 & -3.652700 & 0.326100 \\ \mathrm{H} & 3.355600 & -2.747700 & -1.130500\end{array}$

IVe

$\begin{array}{llll}\text { C } & 0.670700 & 3.151500 & -0.614900\end{array}$

$\begin{array}{llll}\text { C } & -0.084000 & 2.257900 & 0.142700\end{array}$

$\begin{array}{llll}\text { C } & 0.316900 & 0.929400 & 0.174600\end{array}$

$\begin{array}{llll}\text { C } & 1.429600 & 0.488900 & -0.524400\end{array}$

$\begin{array}{llll}\text { C } & 2.179500 & 1.377000 & -1.284800\end{array}$

$\begin{array}{llll}\text { C } & 1.791500 & 2.714900 & -1.323600\end{array}$

$\mathrm{H} \quad 0.384400 \quad 4.198300 \quad-0.653900$

$\begin{array}{llll}\mathrm{H} & -0.959700 & 2.582300 & 0.696100\end{array}$

$\begin{array}{llll}\mathrm{H} & 3.053100 & 1.039600 & -1.836900\end{array}$

$\begin{array}{llll}\mathrm{H} & 2.367700 & 3.425100 & -1.909200\end{array}$

$\begin{array}{llll}\mathrm{C} & 1.608700 & -0.987900 & -0.315600\end{array}$

$\begin{array}{llll}\text { C } & 1.429700 & -1.860000 & -1.566900\end{array}$

$\begin{array}{llll}\text { C } & 2.970800 & -1.448500 & 0.223500\end{array}$

$\mathrm{H} \quad 2.077300 \quad-1.471800 \quad-2.364000$

$\mathrm{H} \quad 0.398300 \quad-1.838900 \quad-1.931300$

$\begin{array}{llll}\mathrm{H} & 3.760800 & -0.886800 & -0.288300\end{array}$

$\begin{array}{llll}\mathrm{H} & 3.049000 & -1.231800 & 1.291400\end{array}$

$\begin{array}{llll}\mathrm{O} & 0.595100 & -1.331800 & 0.650000\end{array}$

$\begin{array}{llll}\text { C } & -0.298500 & -0.256300 & 0.892600\end{array}$

$\mathrm{H} \quad-0.354300 \quad-0.072600 \quad 1.974300$

$\begin{array}{llll}\text { C } & -1.663500 & -0.576000 & 0.378500\end{array}$

$\mathrm{H} \quad-1.779200 \quad-1.324900 \quad-0.396700$

$\begin{array}{llll}\text { C } & -2.813500 & 0.148300 & 0.870500\end{array}$

$\begin{array}{llll}\mathrm{O} & -2.761600 & 1.042400 & 1.702200\end{array}$

$\begin{array}{llll}\mathrm{O} & -3.964200 & -0.268100 & 0.304800\end{array}$

$\begin{array}{llll}\text { C } & -5.134700 & 0.414200 & 0.744500\end{array}$

$\begin{array}{llll}\mathrm{H} & -5.067300 & 1.482000 & 0.519200\end{array}$ 


$\begin{array}{lrrr}\mathrm{H} & -5.961900 & -0.038900 & 0.198600 \\ \mathrm{H} & -5.273600 & 0.289600 & 1.821700 \\ \mathrm{C} & 1.901400 & -3.242000 & -1.101900 \\ \mathrm{H} & 2.202600 & -3.885100 & -1.933800 \\ \mathrm{H} & 1.081300 & -3.741400 & -0.577600 \\ \mathrm{C} & 3.063100 & -2.955600 & -0.115000 \\ \mathrm{H} & 2.969200 & -3.570100 & 0.784100 \\ \mathrm{H} & 4.033000 & -3.192100 & -0.563400\end{array}$




\section{References}

(1) F. L. Vaillant, M. Garreau, S. Nicolai, G. Gryn'ova, C. Corminboeuf, J. Waser, Chem. Sci. 2018, 9, 5883.

(2) J. Luo, J. Zhang, ACS Catal. 2016, 6, 873-877.

(3) E. Ota, H. Wang, N. L. Frye, R. R. Knowles, J. Am. Chem. Soc. 2019, 141, 1457.

(4) T. Yasui, T. Kikuchi, Y. Yamamoto, Chem. Commun. 2020, 56, 12865.

(5) P. P. Jadhav, N. M. Kahar, S. G. Dawande, Eur. J. Org. Chem. 2019, 7831.

(6) S. Roy, S. K. Das, B. Chattopadhyay, Angew. Chem. Int. Ed. 2018, 57, 2238.

(7) G. -Z. Wang, R. Shang, Y. Fu, Org. Lett. 2018, 20, 888.

(8) A. Abad, C. Agulló, A. C. Cuñat, D. Jiménez, R. H. Perni, Tetrahedron 2001, 57, 9727.

(9) X. Hu, Y. Dong, G. Liu, Mol. Divers. 2015, 19, 695.

(10) P. Cazeau, F. Duboudin, F. Moulines, O. Babot, J. Dunogues, Tetrahedron 1987, 43, 2075.

(11) Q. Li, J. Liu, Y, Wei, M. Shi, J. Org. Chem. 2020, 85, 2438.

(12) J. Li, X. Chi, L. Meng, L. Jiao, W. Shang, P. Wang, D. Zhang, Y. Dong, Q. Liu, H. Liu, Org. Biomol. Chem. 2018, 16, 7356.

(13) T. Osako, M. Nagaosa, G. Hamasaka, Y. Uozumi, Synlett 2018, 29, 2251.

(14) W. Wu, Z. Lin, C. Zhu, P. Chen, J. Li, H. Jiang, J. Org. Chem. 2017, 82, 12746.

(15) J. Takagi, K. Takahashi, T. Ishiyama, N. Miyaura, J. Am. Chem. Soc. 2002, 124, 8001.

(16) R. L. Danheiser, A. L. Helgason, J. Am. Chem. Soc. 1994, 116, 9471.

(17) Y. Sajja, H. R. Vulupala, R. Bantu, L. Nagarapu, S. B. Vasamsetti, S. Kotamraju, J. B. Nanubolu, Bioorg. Med. Chem. Lett. 2016, 26, 858.

(18) N. Ishida, S. Sawano, M. Murakami, Chem. Commun. 2012, 48, 1973.

(19) H. Kinoshita, K. Yaguchi, T. Tohjima, N. Hirai, K. Miura, Tetrahedron Letters 2016, 57, 203.

(20) Gaussian 16, Revision A.03, M. J. Frisch, G. W. Trucks, H. B. Schlegel, G. E. Scuseria, M. A. Robb, J. R. Cheeseman, G. Scalmani, V. Barone, G. A. Petersson, H. Nakatsuji, X. Li, M. Caricato, A. V. Marenich, J. Bloino, B. G. Janesko, R. Gomperts, B. Mennucci, H. P. Hratchian, J. V. Ortiz, A. F. Izmaylov, J. L. Sonnenberg, D. Williams-Young, F. Ding, F. Lipparini, F. Egidi, J. Goings, B. Peng, A. Petrone, T. Henderson, D. Ranasinghe, V. G. Zakrzewski, J. Gao, N. Rega, G. Zheng, W. Liang, M. Hada, M. Ehara, K. Toyota, R. Fukuda, J. Hasegawa, M. Ishida, T. Nakajima, Y. Honda, O. Kitao, H. Nakai, T. Vreven, K. Throssell, J. A. Montgomery, Jr., J. E. Peralta, F. Ogliaro, M. J. Bearpark, J. J. Heyd, E. N. Brothers, K. N. Kudin, V. N. Staroverov, T. A. Keith, R. Kobayashi, J. Normand, K. Raghavachari, A. P. Rendell, J. C. Burant, S. S. Iyengar, J. Tomasi, M. Cossi, J. M. Millam, M. Klene, C. Adamo, R. Cammi, J. W. Ochterski, R. L. Martin, K. Morokuma, O. Farkas, J. B. Foresman, and D. J. Fox, Gaussian, Inc., Wallingford CT, 2016.

(21) J.-D. Chai, M. Head-Gordon, Phys. Chem. Chem. Phys. 2008, 10, 661574-6620.

(22) (a) W. J. Hehre, R. Ditchfield, J. A. Pople, J. Chem. Phys. 1972, 56, 2257-2261. (b) P. C. Hariharan, J. A. Pople, Theor. Chim. Acta 1973, 28, 213-222. (c) M. M. Fracl, W. J. Pietro, W. J. Hehre, J. S. Binkley, M. S. Gordon, D. J. DeFrees, J. A. Pople, J. Chem. Phys. 1982, 77, 3654-3665.

(23) (a) K. Fukui, Acc. Chem. Res. 1981, 14, 363-368. (b) C. Gonzalez, H. B. Schlegel, J. Chem. Phys. 1989, 90, 2154-2161. (c) C. Gonzalez, H. B. Schlegel, J. Phys. Chem. 1990, 94, 5523-5527.

(24) (a) R. Krishnan, J. S. Binkley, R. Seeger, J. A. Pople, J. Chem. Phys. 1980, 72, 650-654. (b) A. D. McLean, G. S. Chandler, J. Chem. Phys. 1980, 72, 5639-5648. (c) M. J. Frisch, J. A. Pople, J. S. Binkley, J. Chem. Phys. 1984, 80, 3265-3269. (d) T. Clark, J. Chandrasekhar, G. W. Spitznagel, P. v. R. Schleyer, J. Comp. 
Chem. 1983, 4, 294-301.

(25) A. V. Marenich, C. J. Cramer, D. G. Truhlar, J. Phys. Chem. B 2009, 113, 6378-6396.

CYLview, 1.0b; Legault, C. Y., Université de Sherbrooke, 2009 (http://www.cylview.org). 


\section{NMR Spectra}
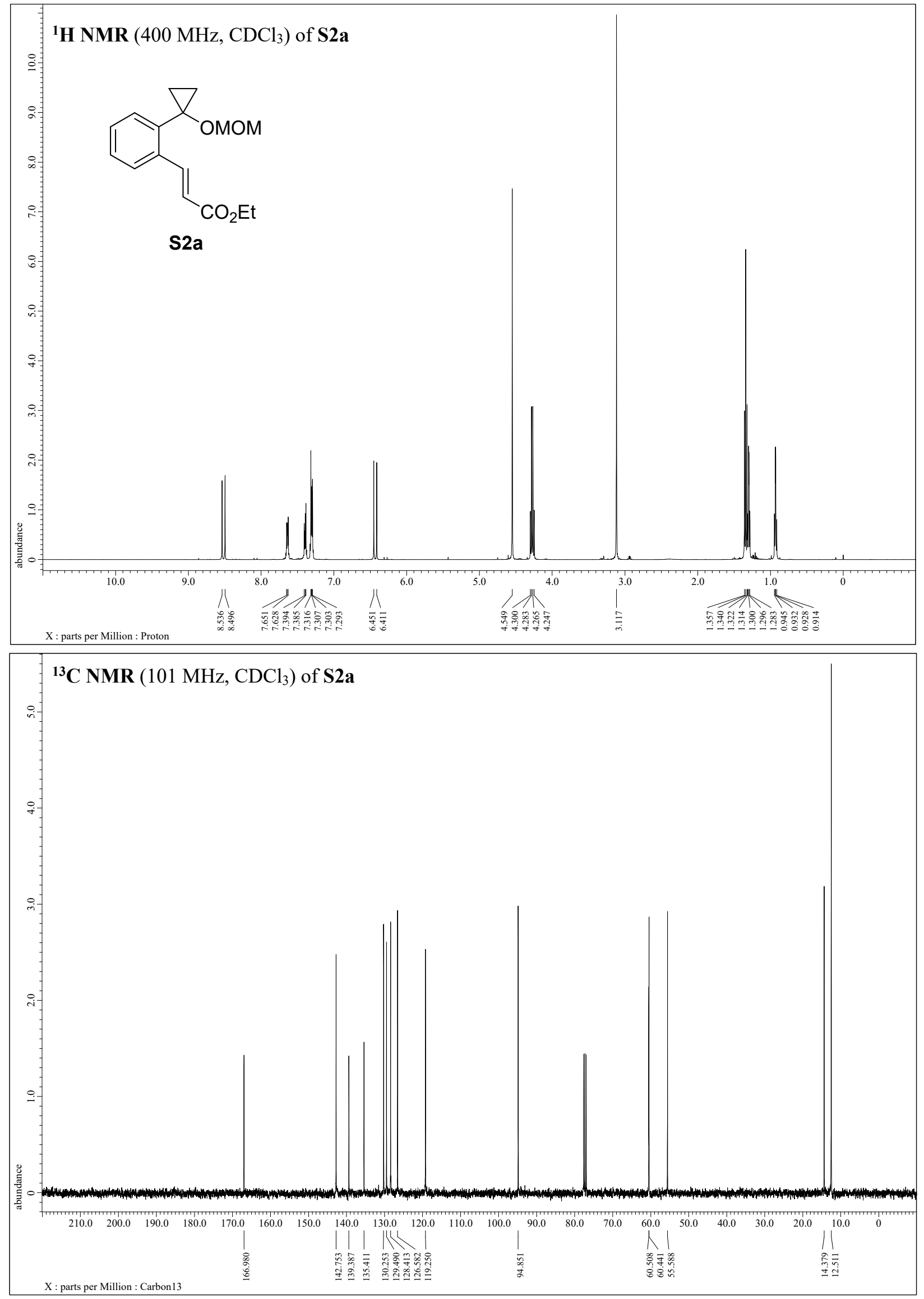

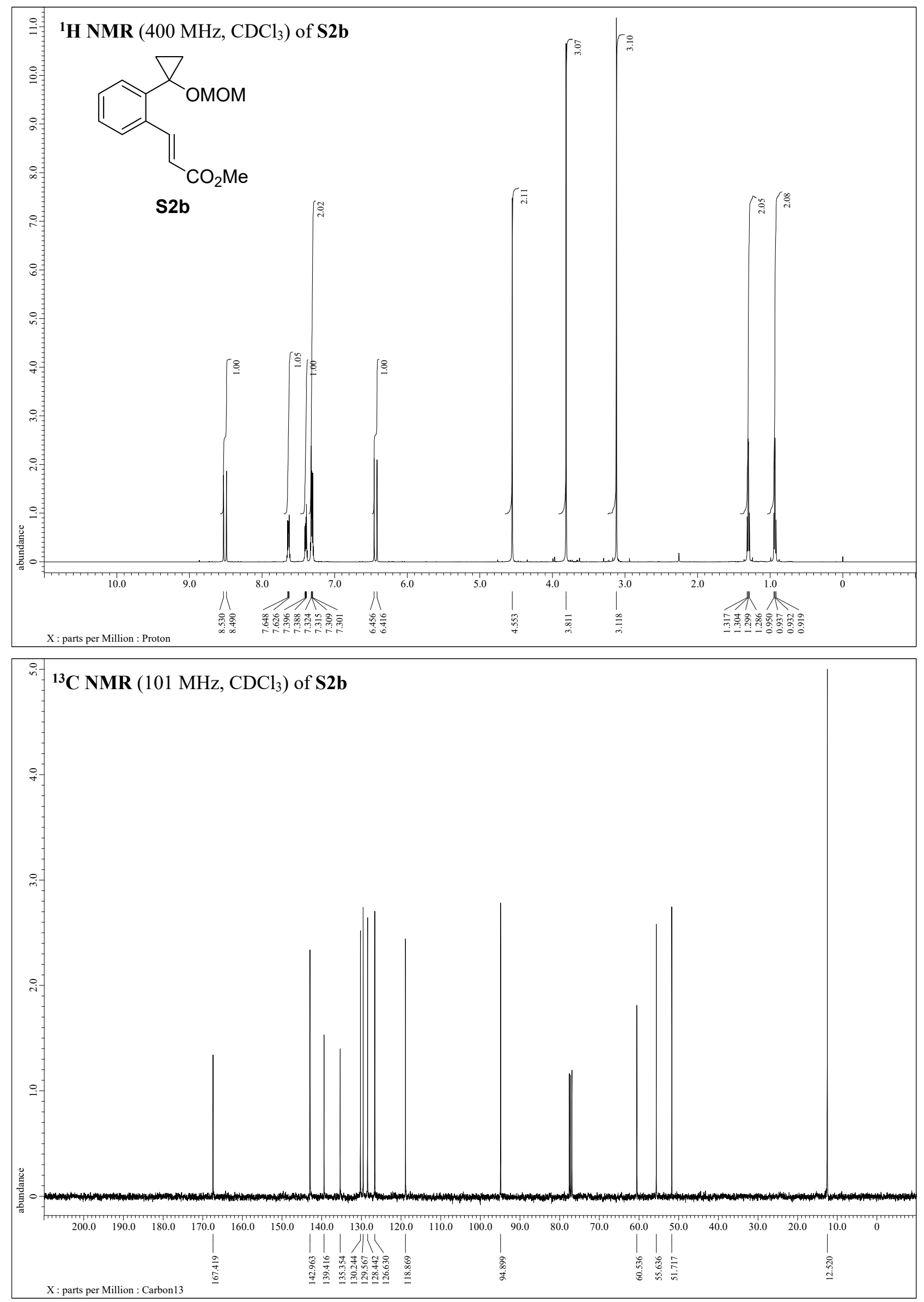

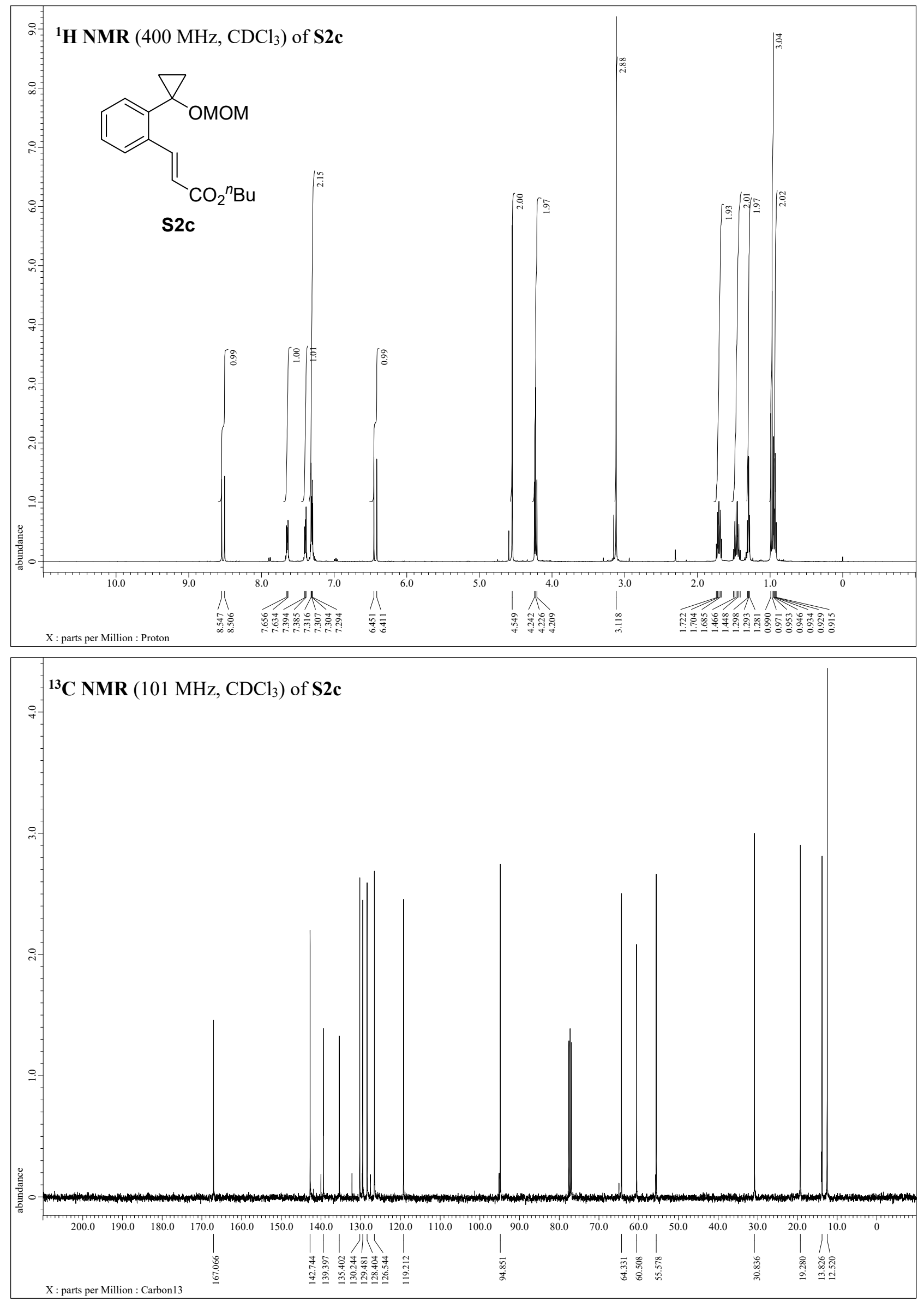

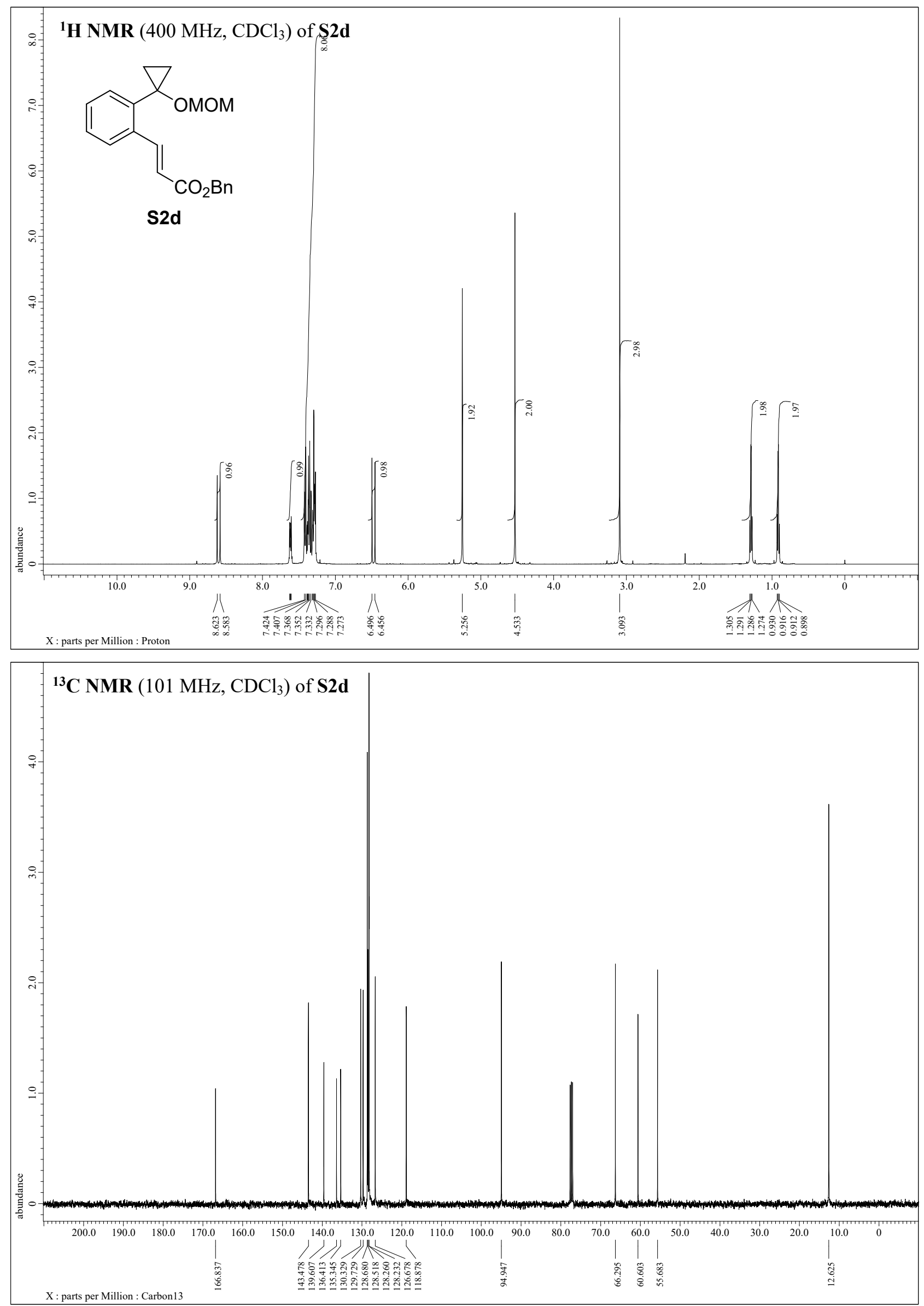

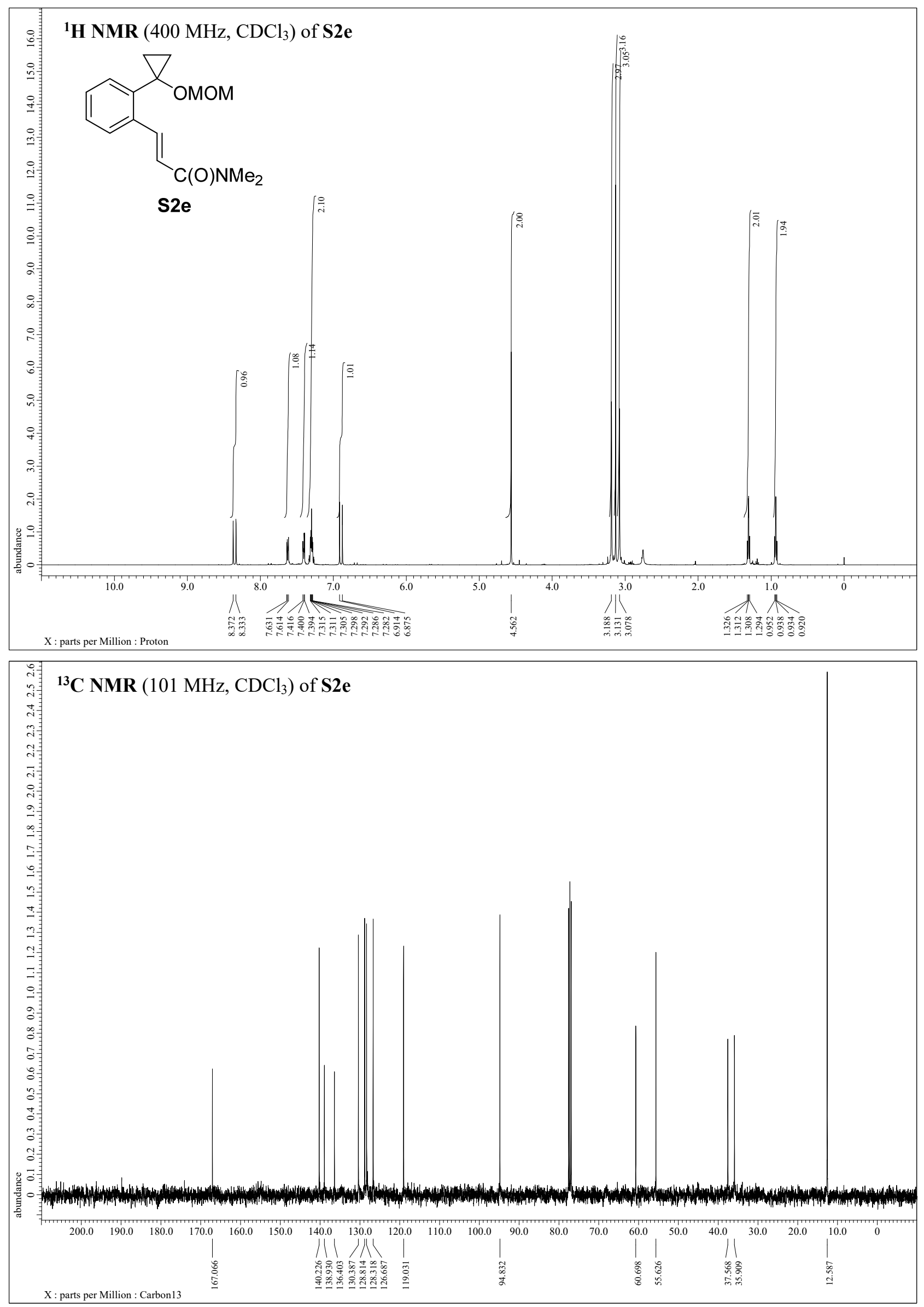

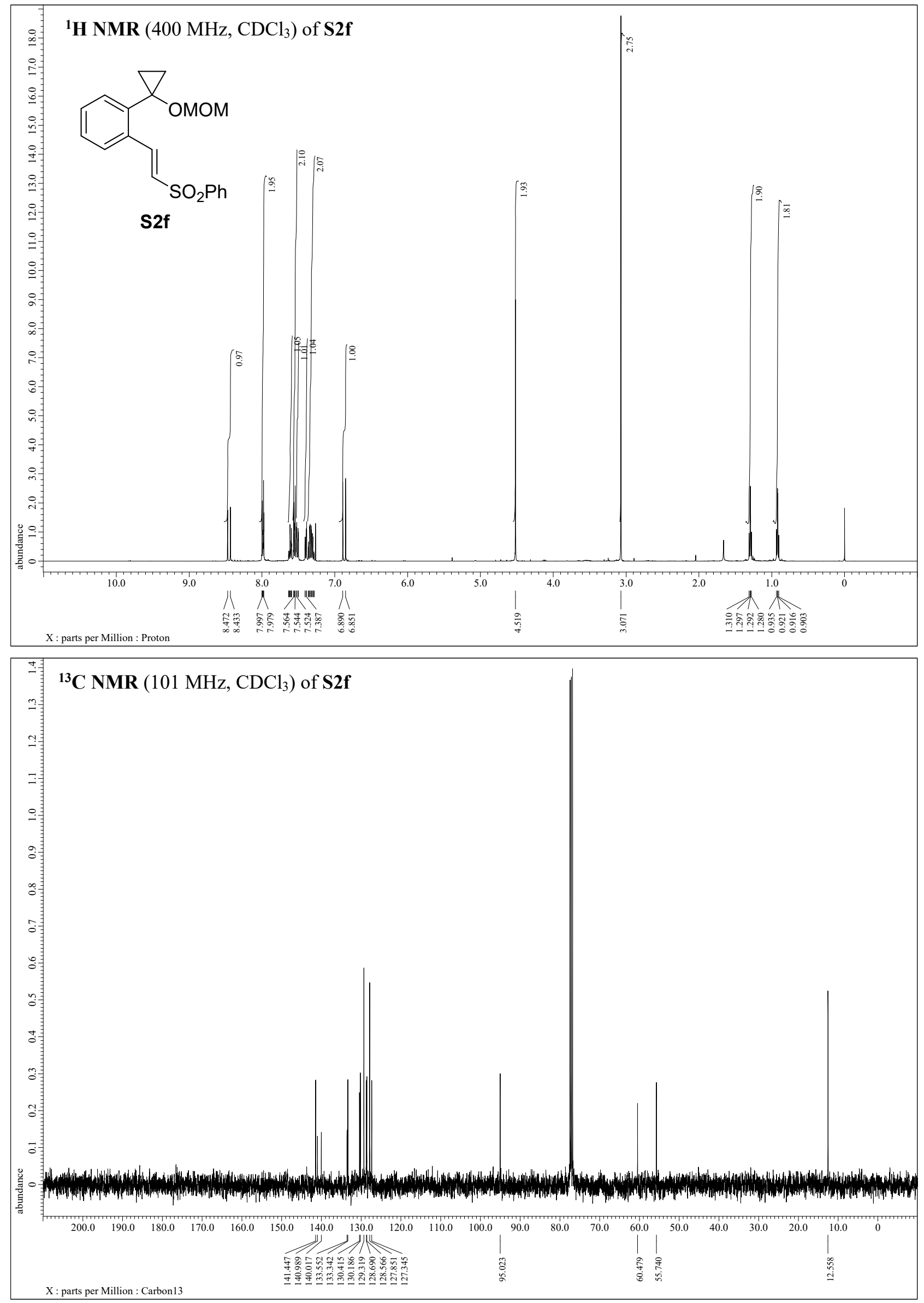

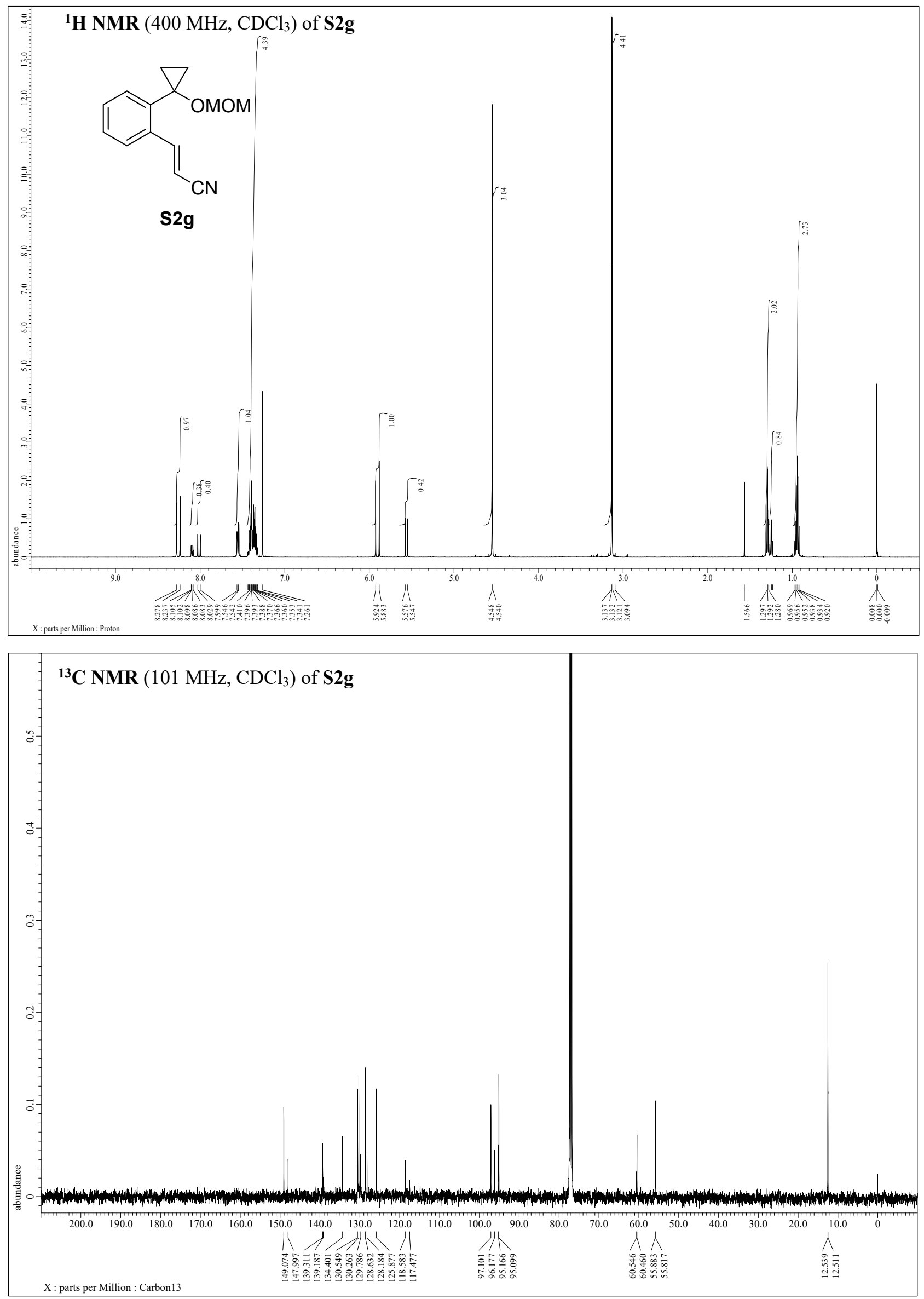

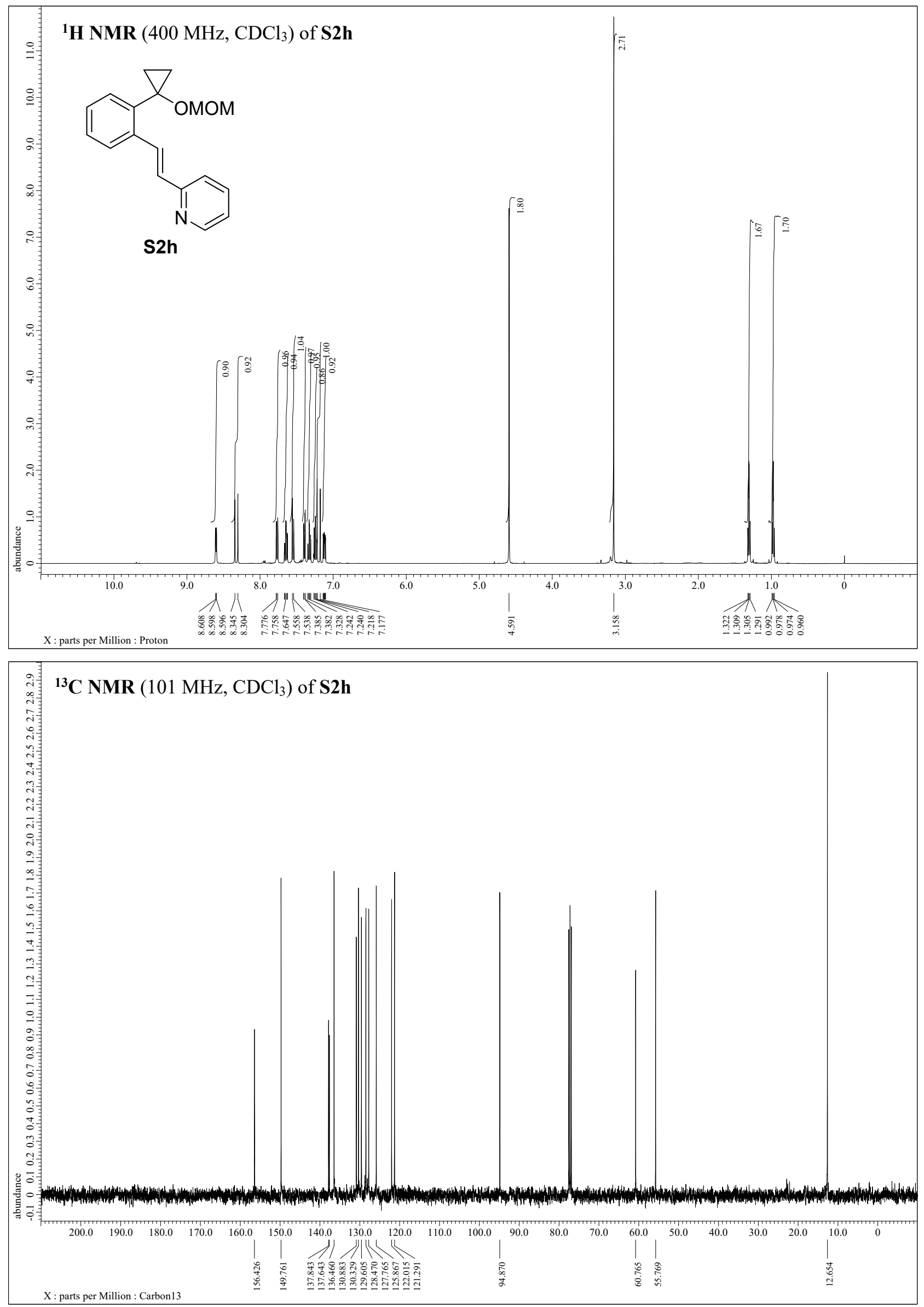

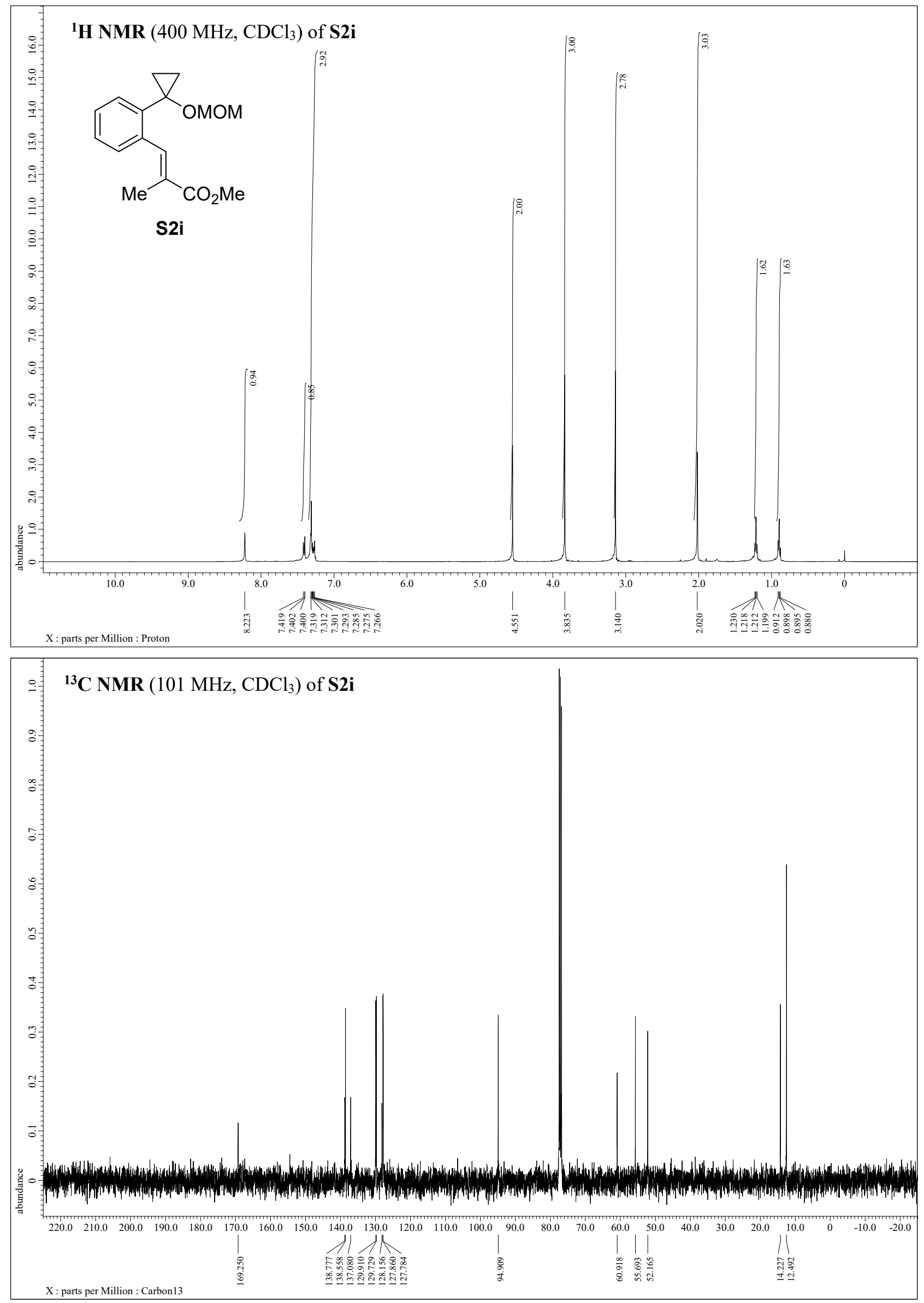

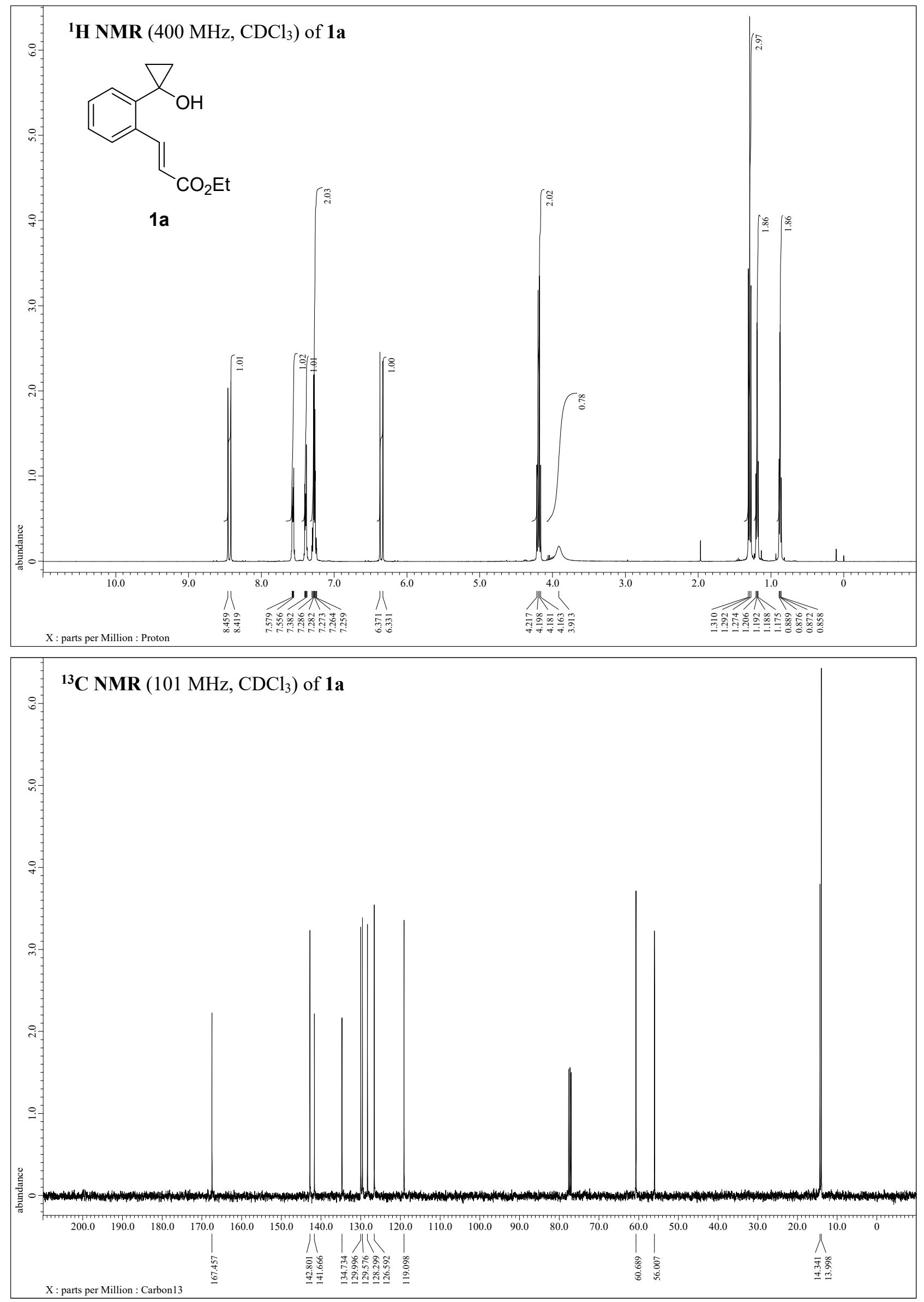

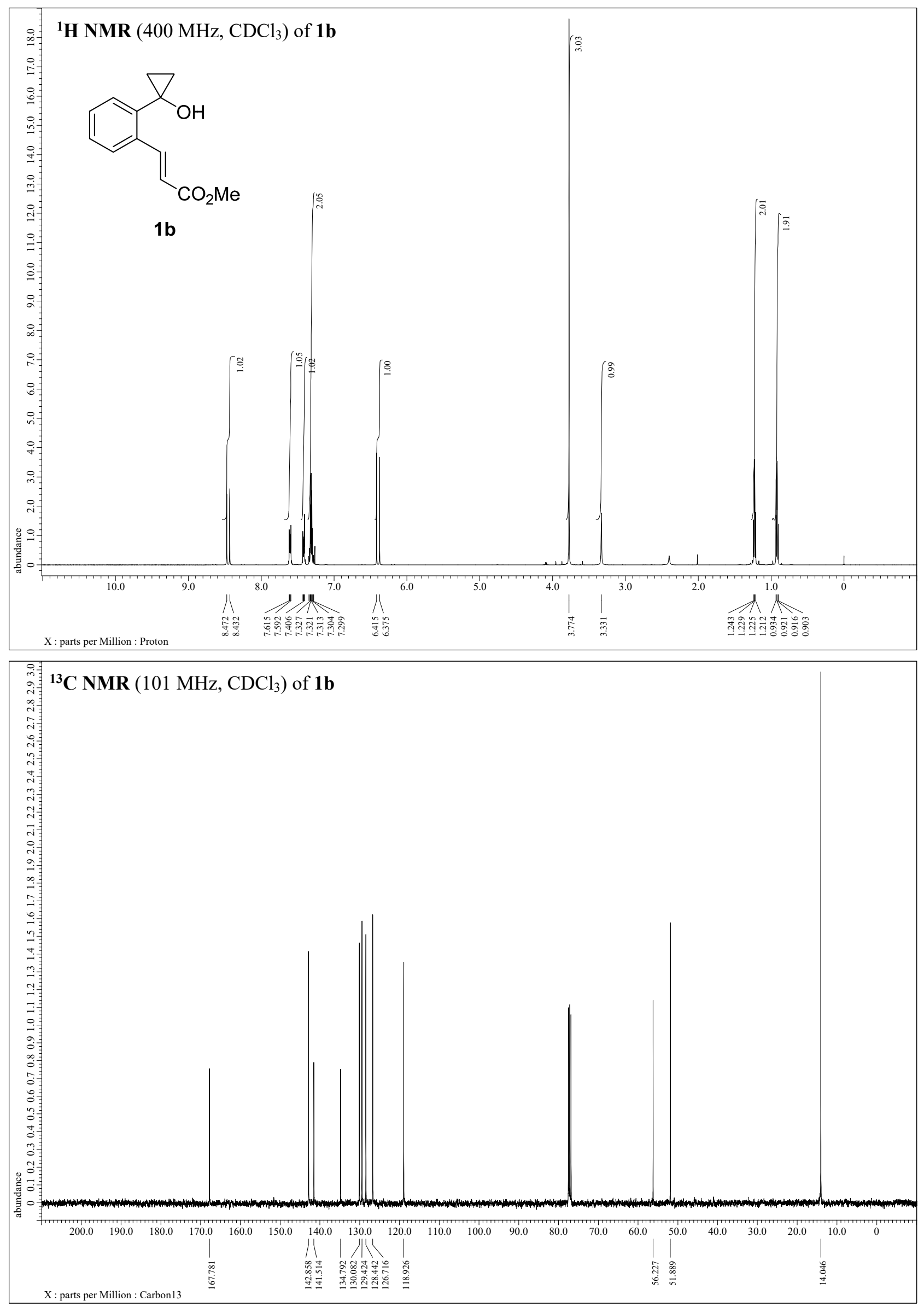

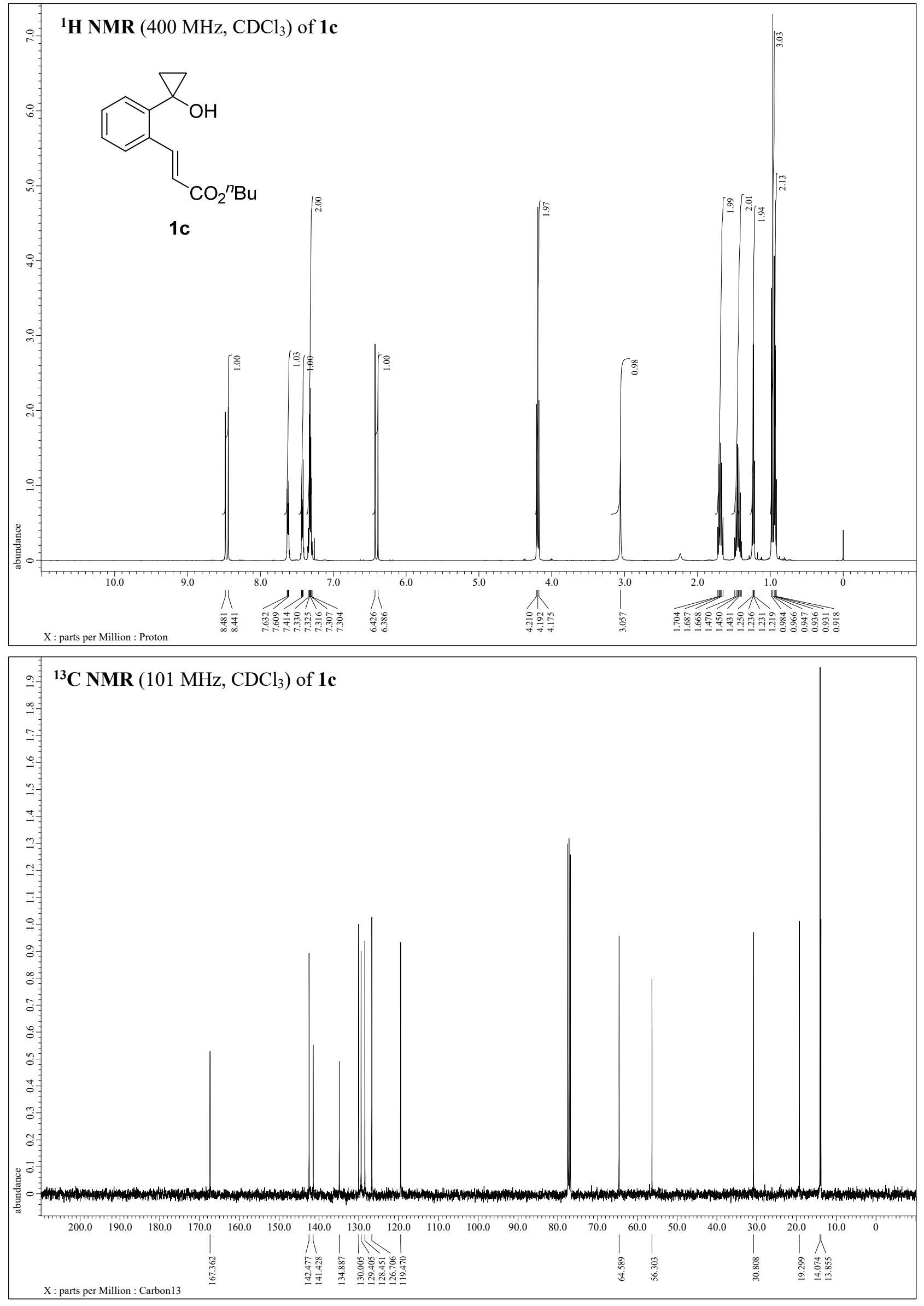

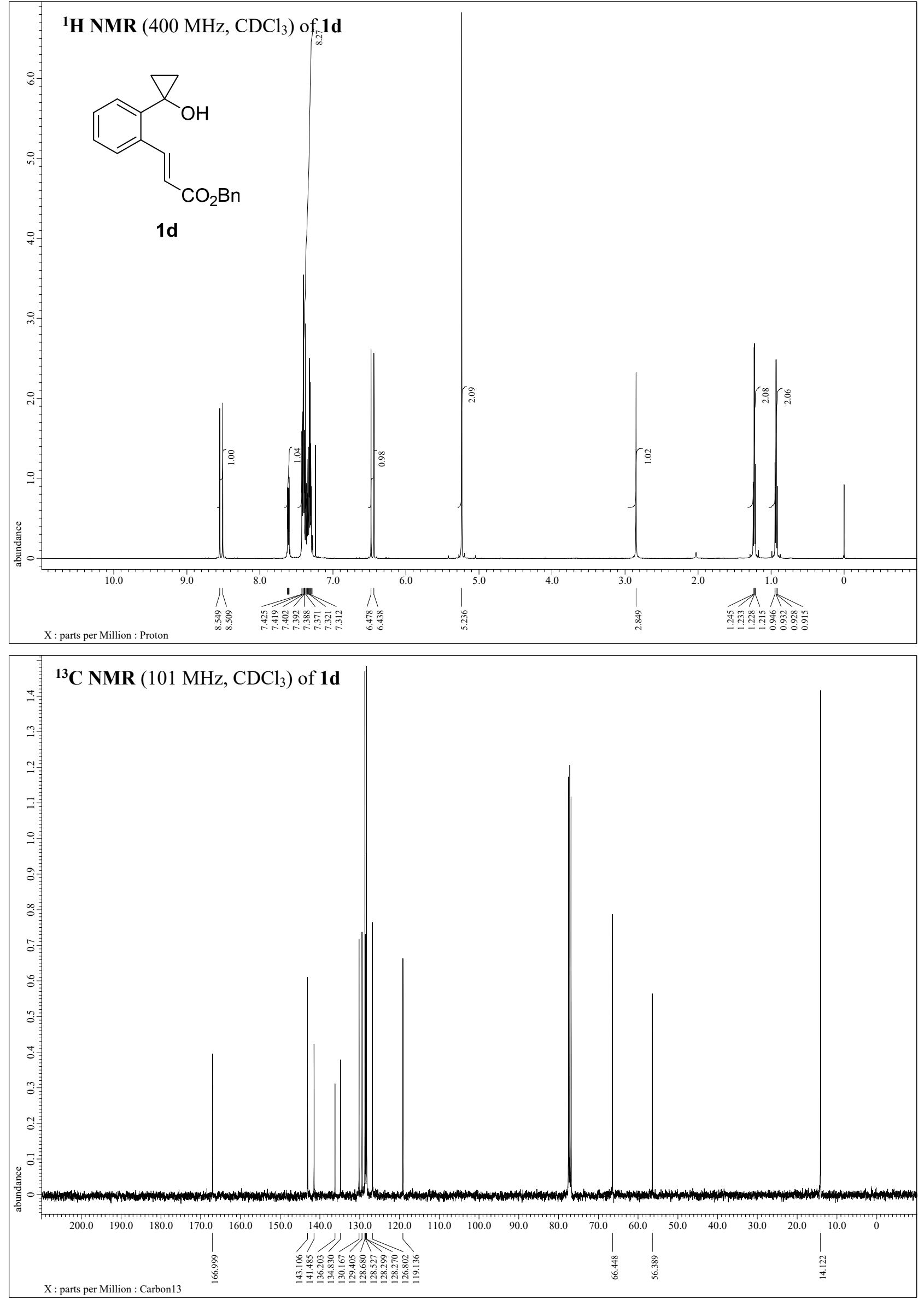

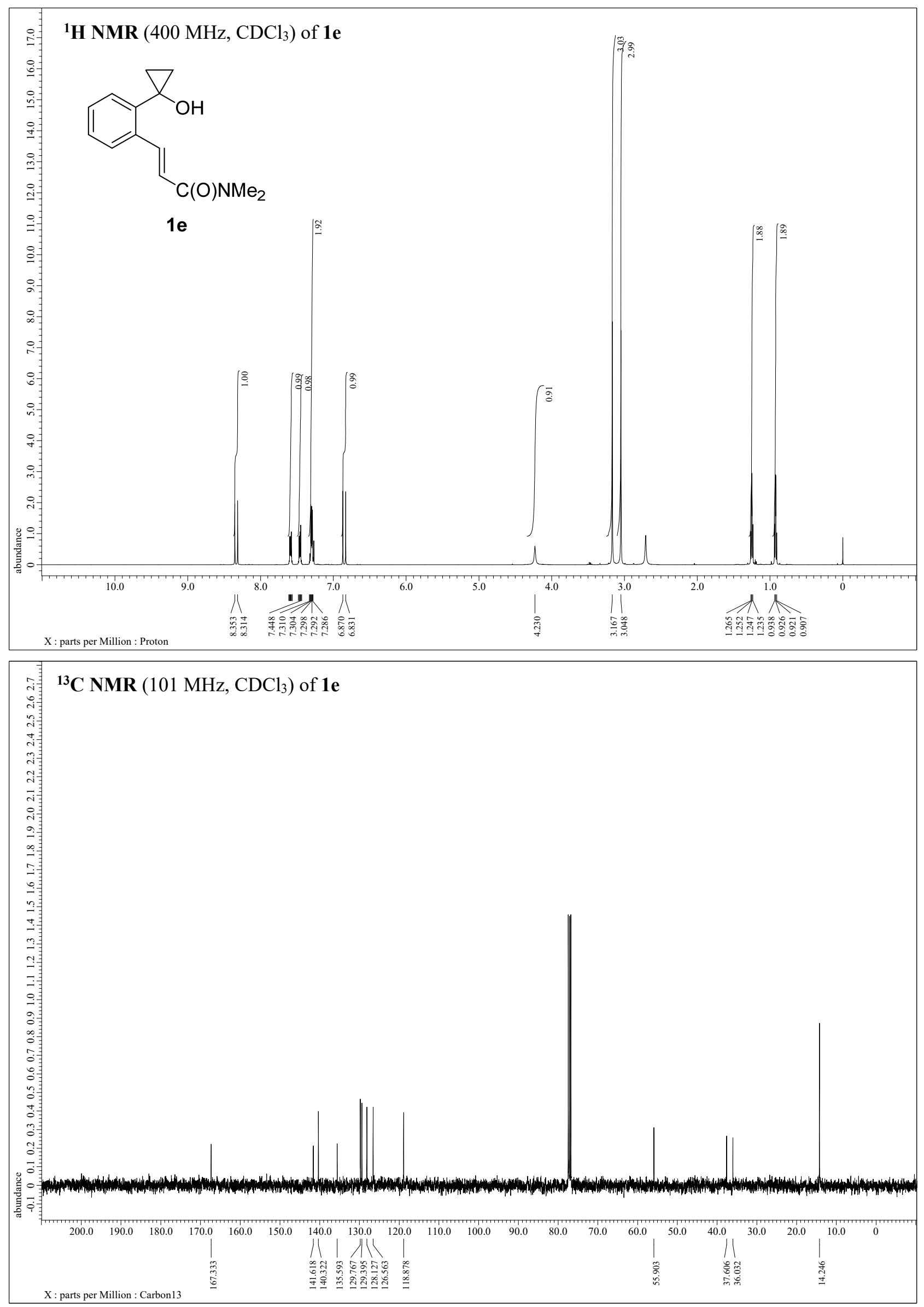

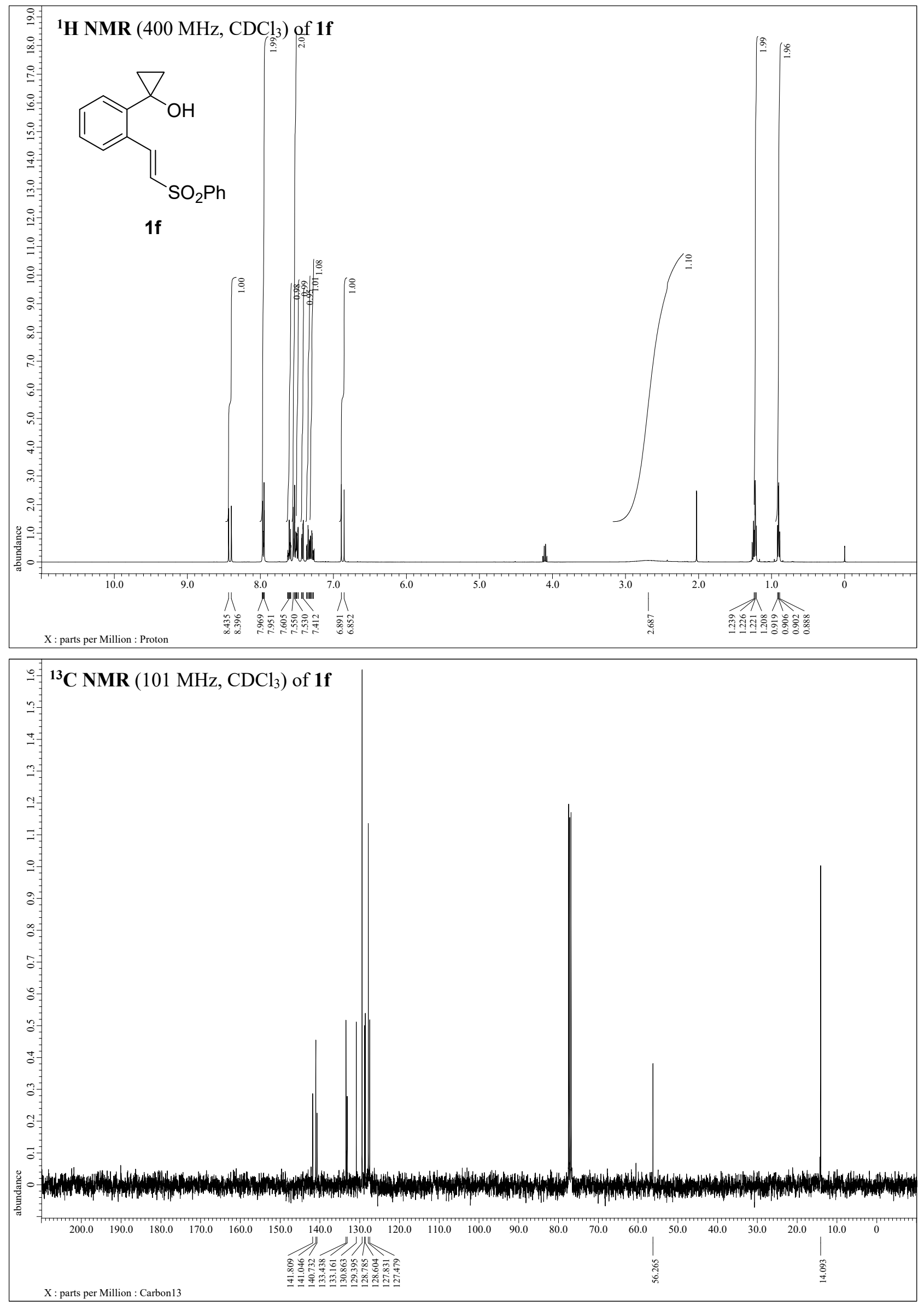

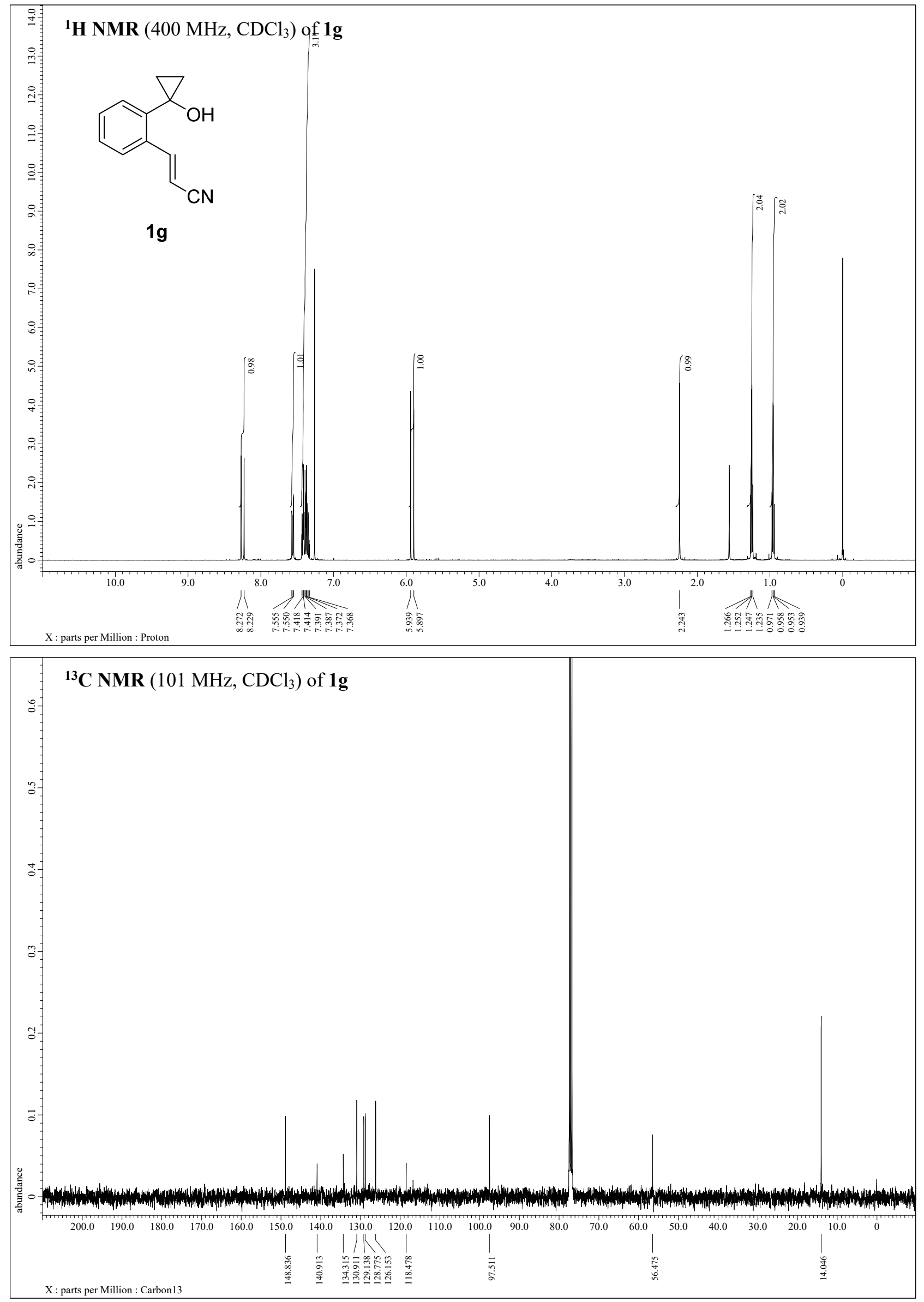

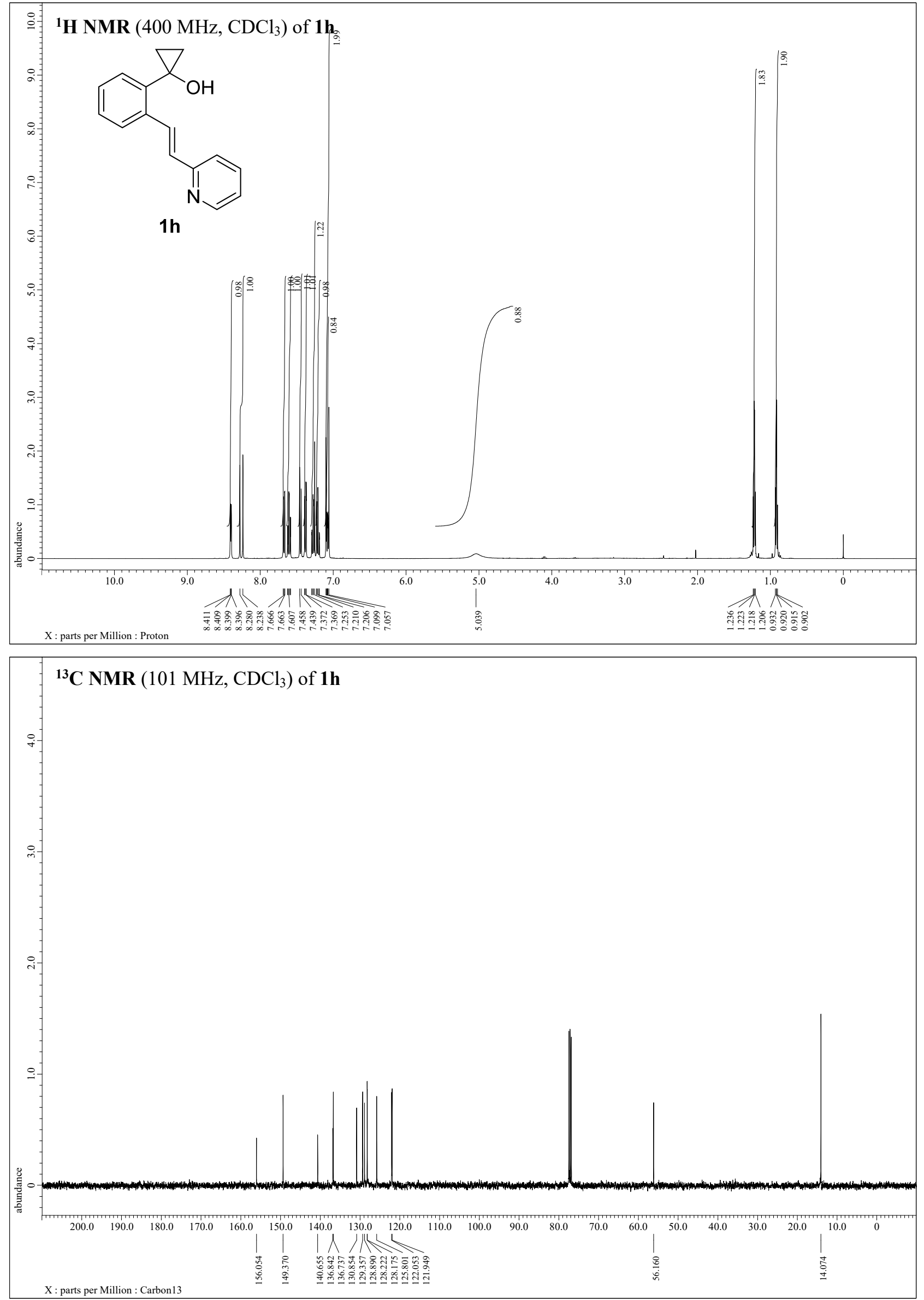

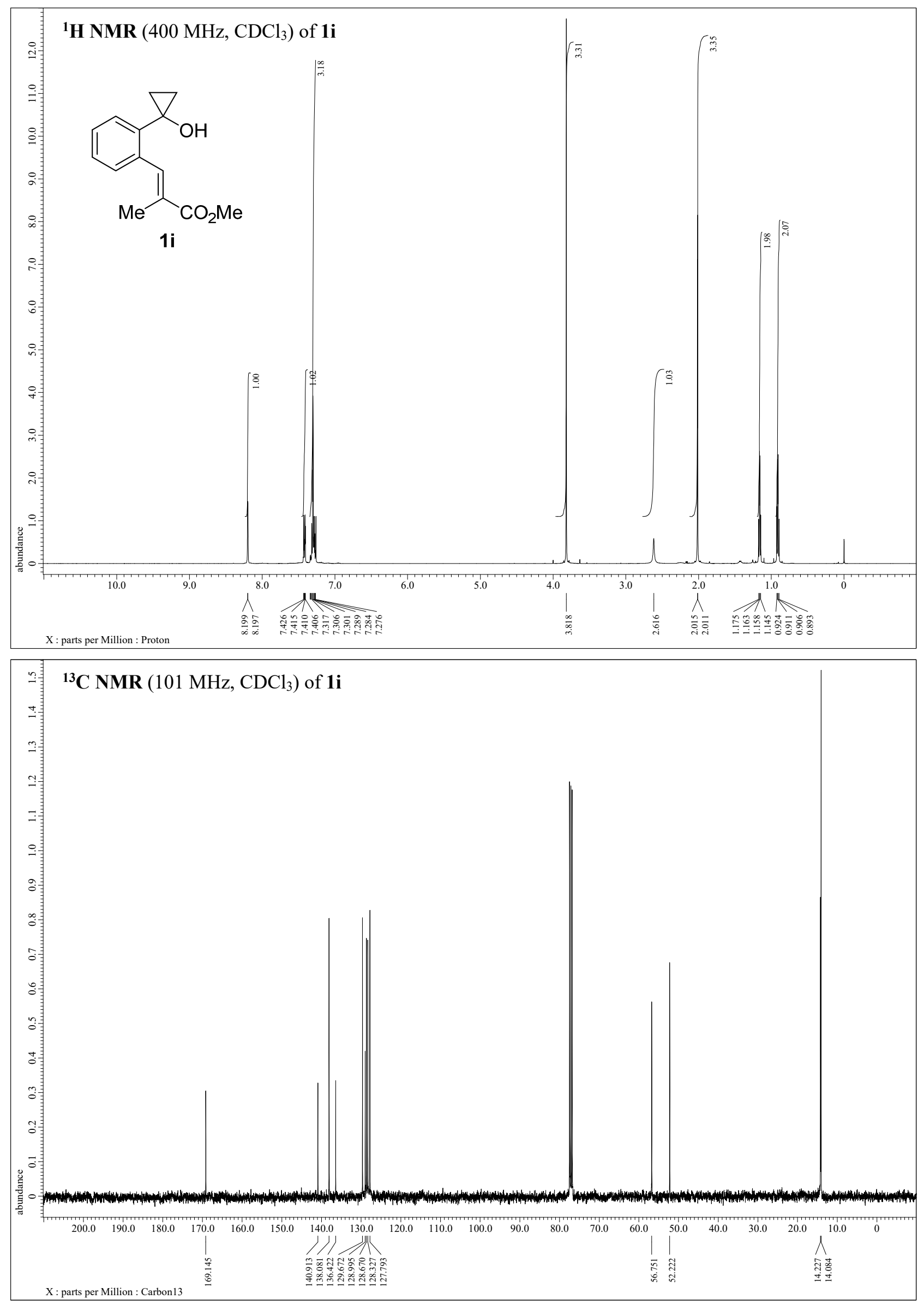

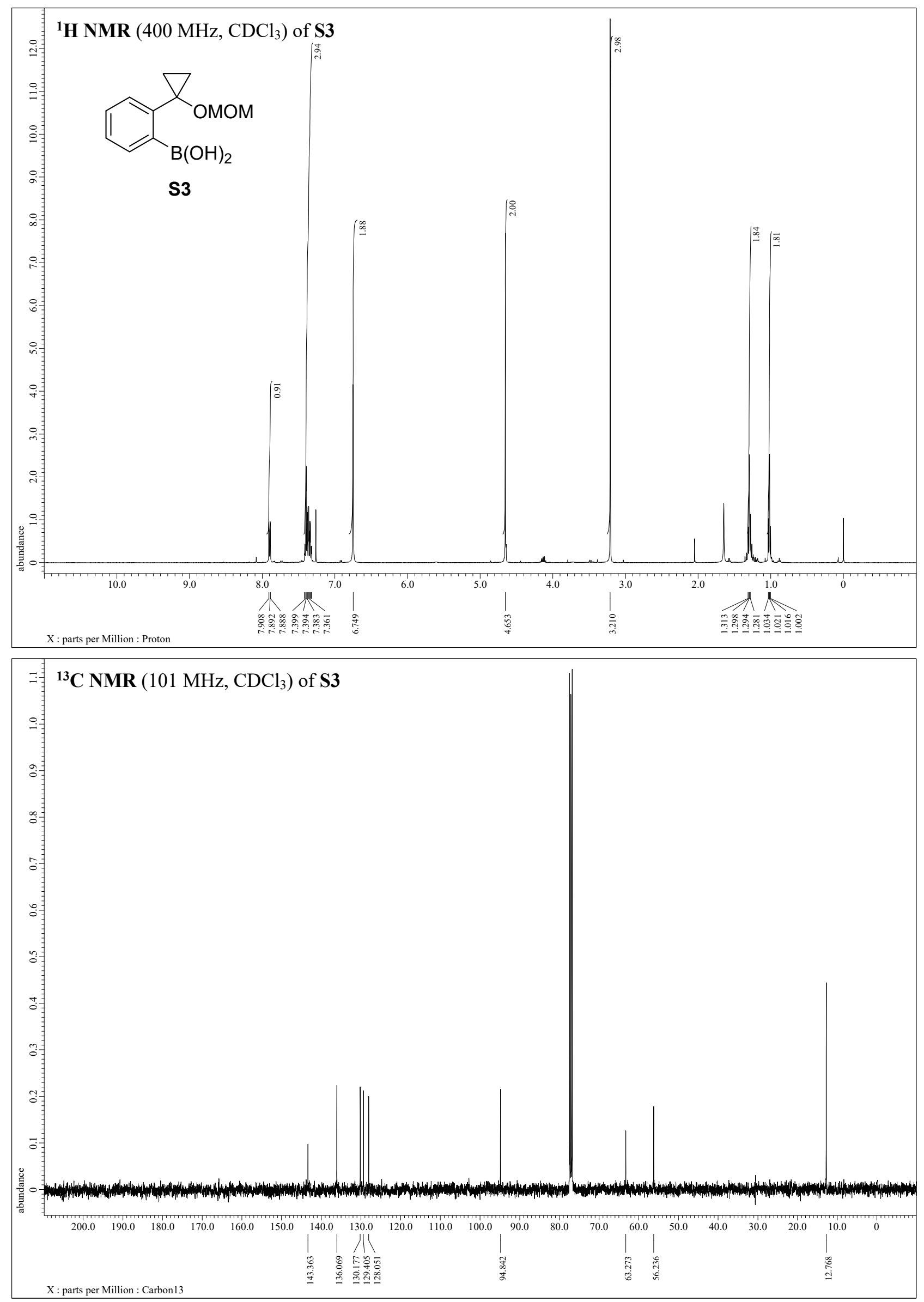

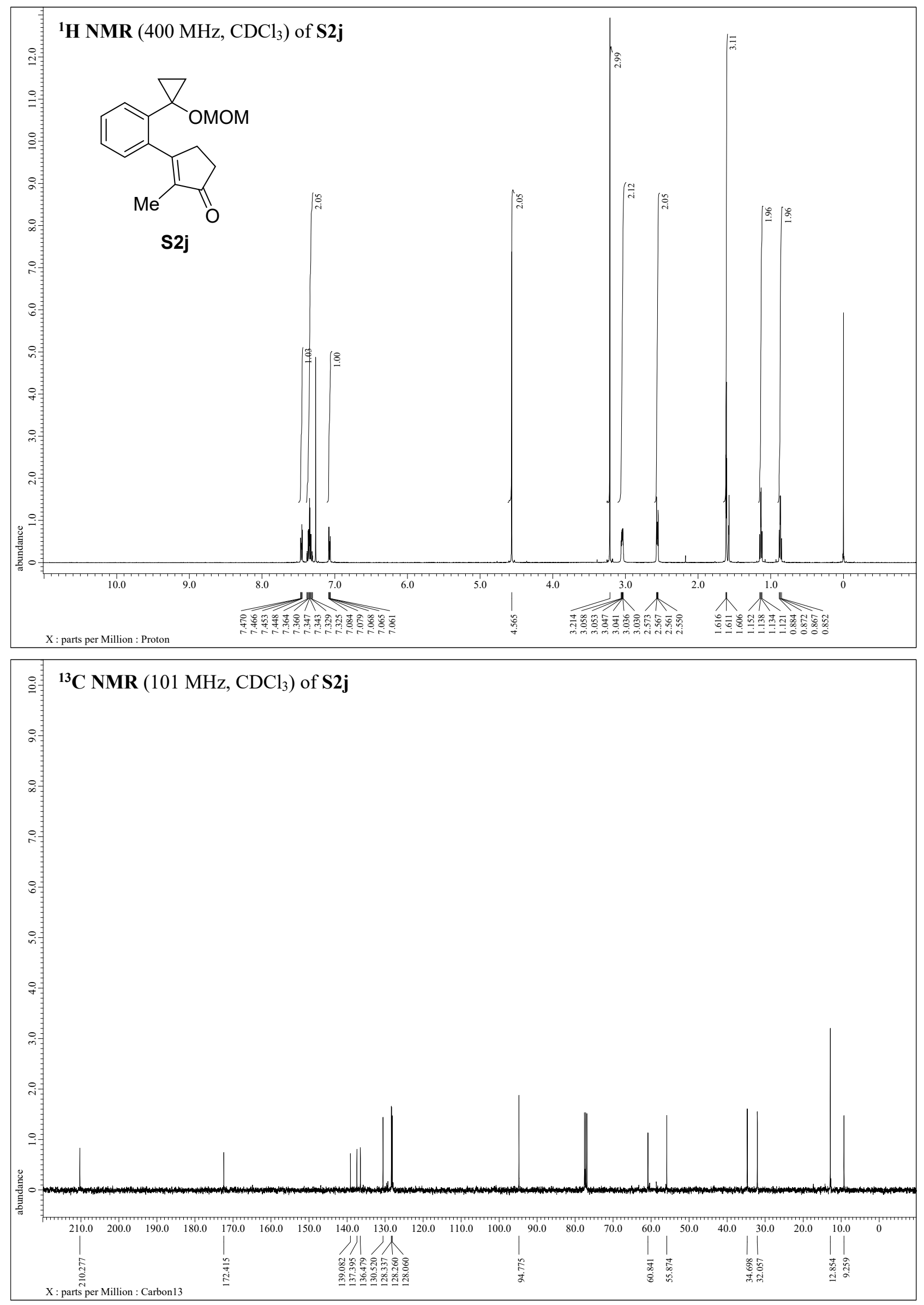

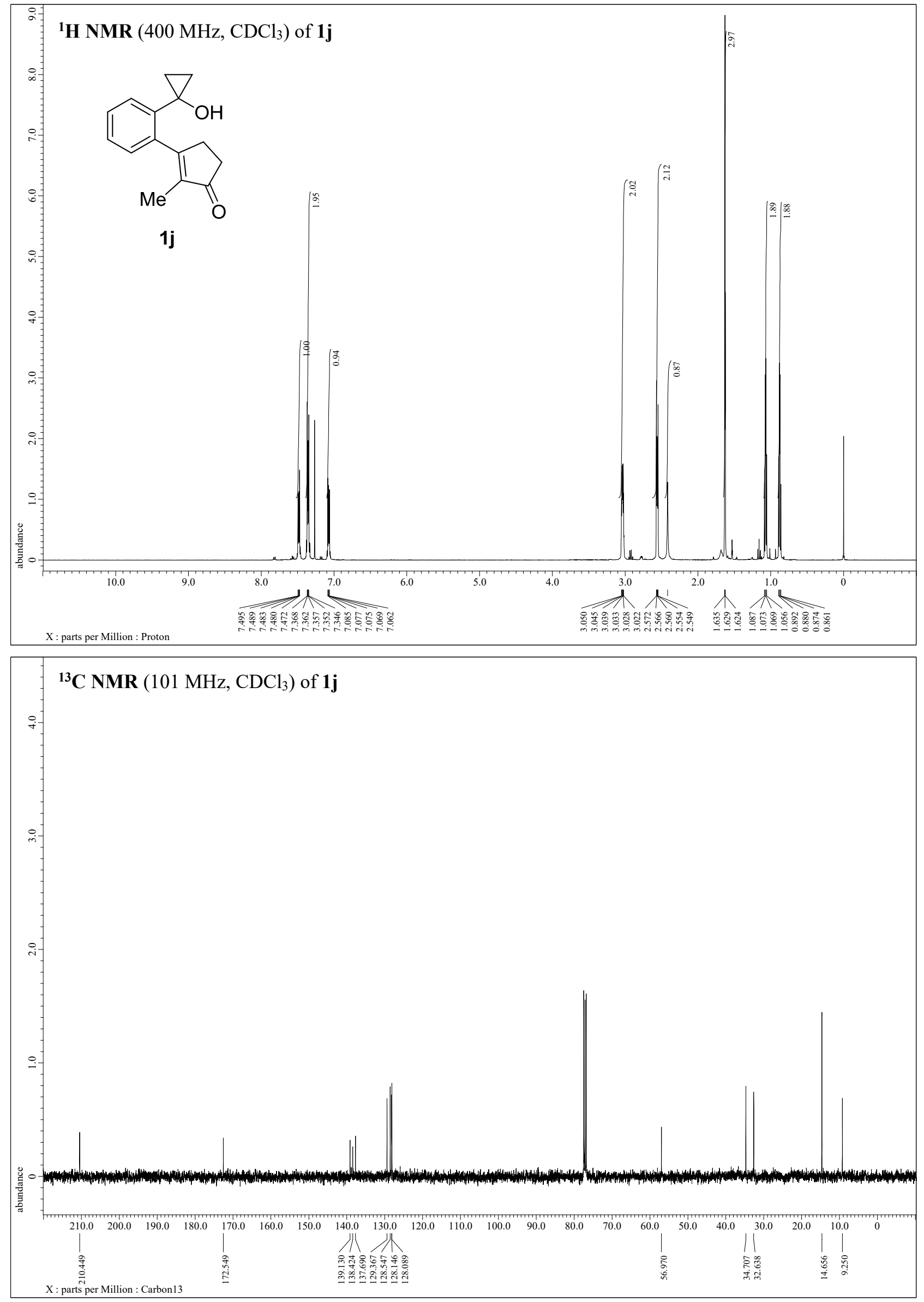

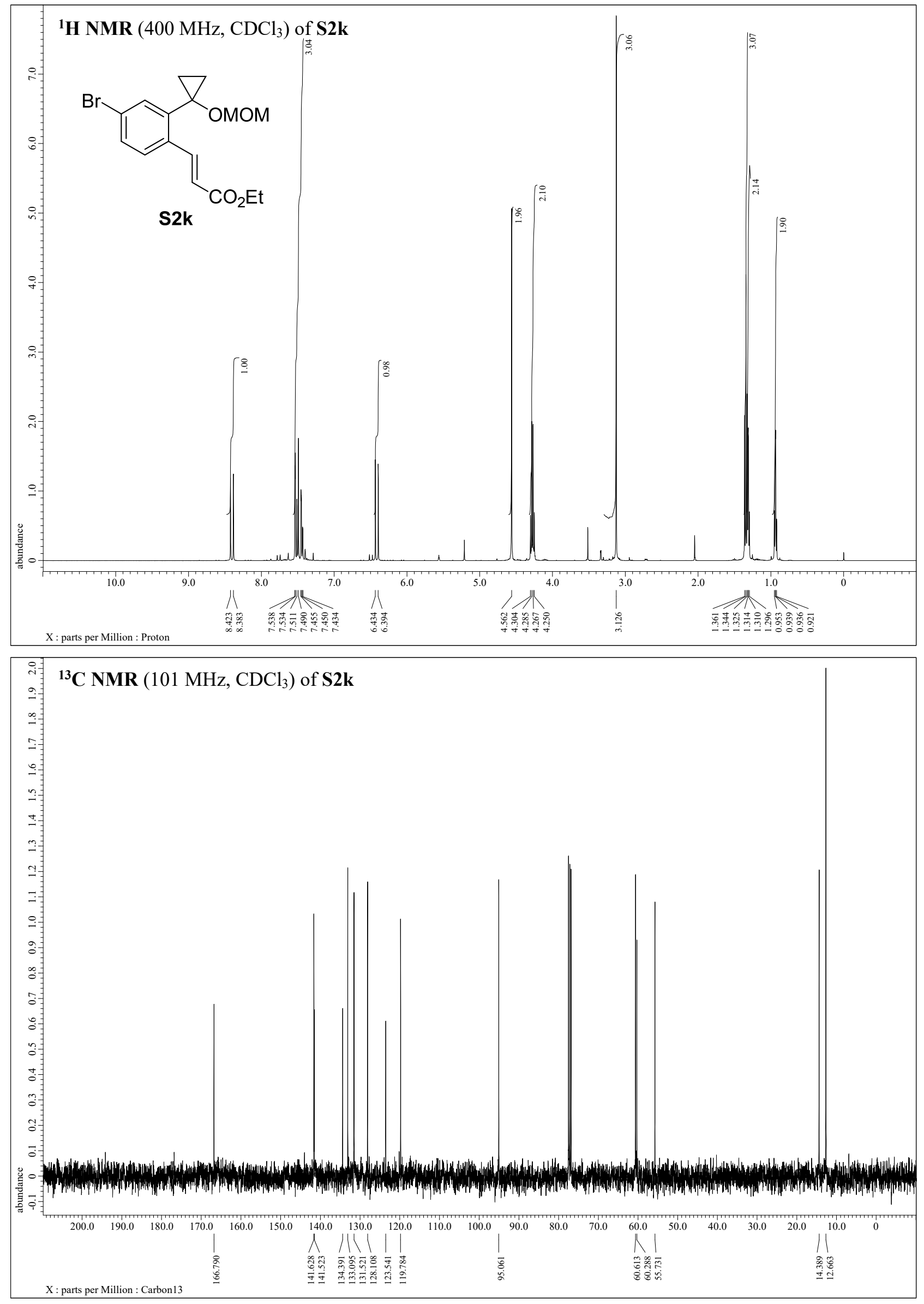

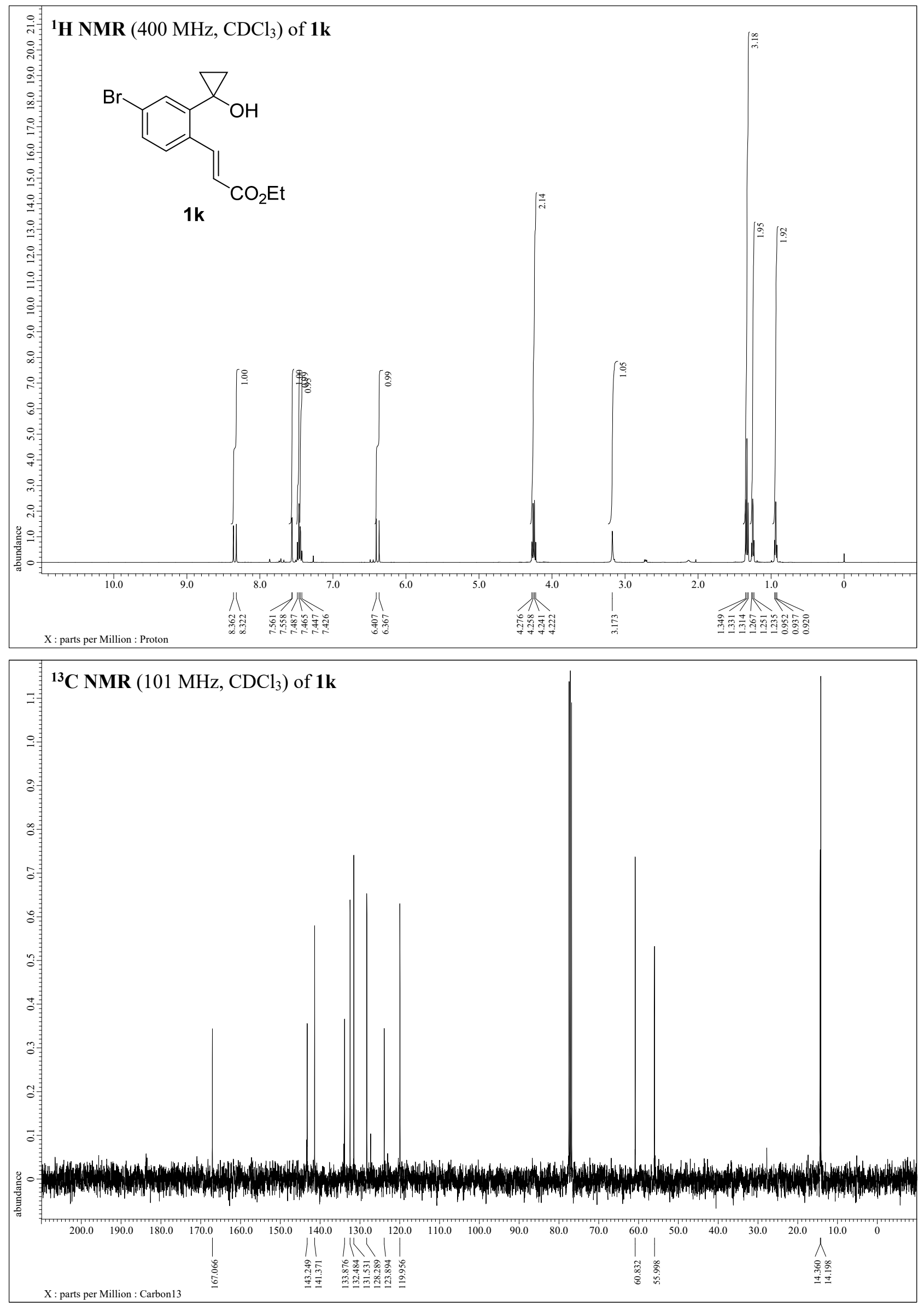

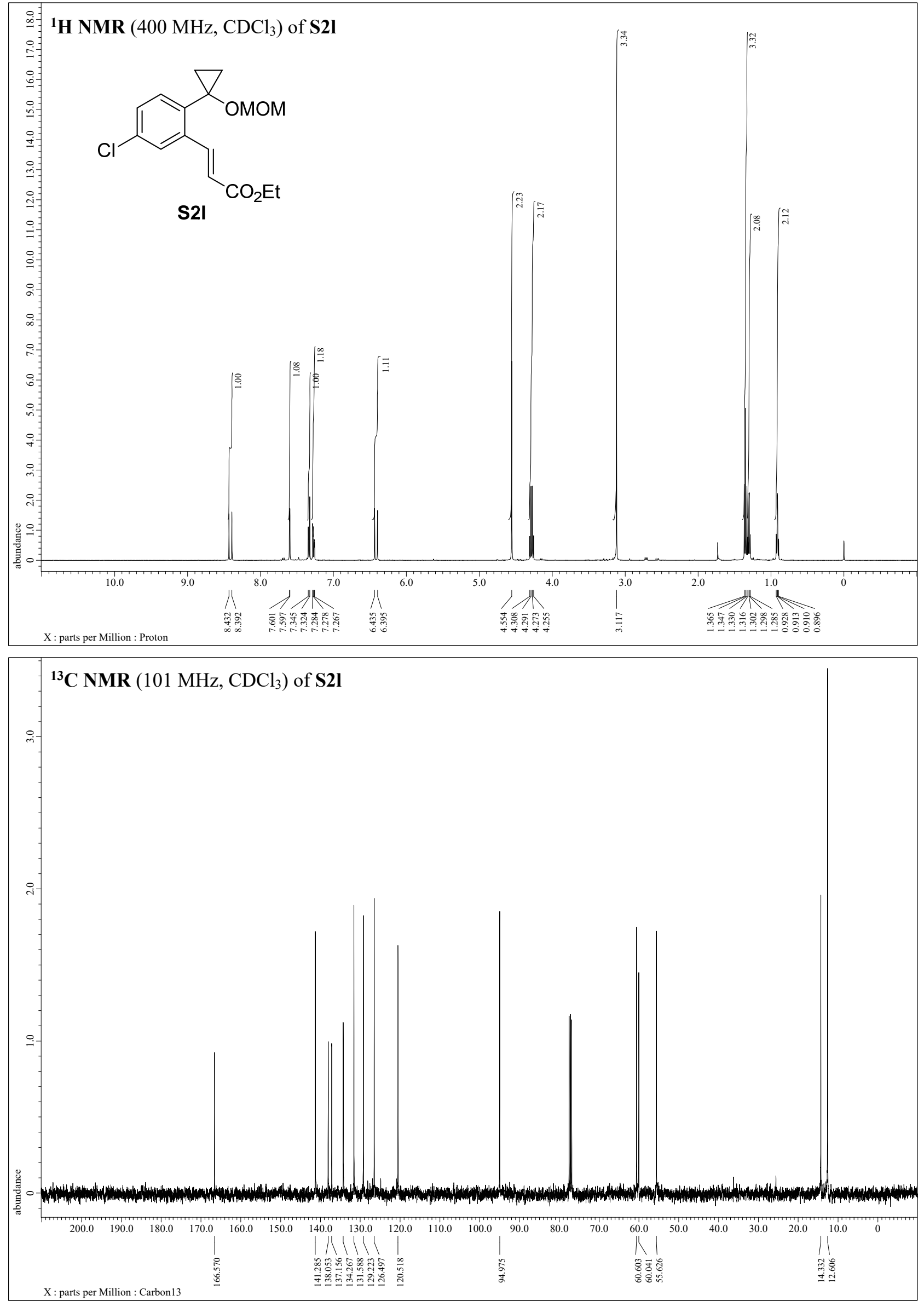

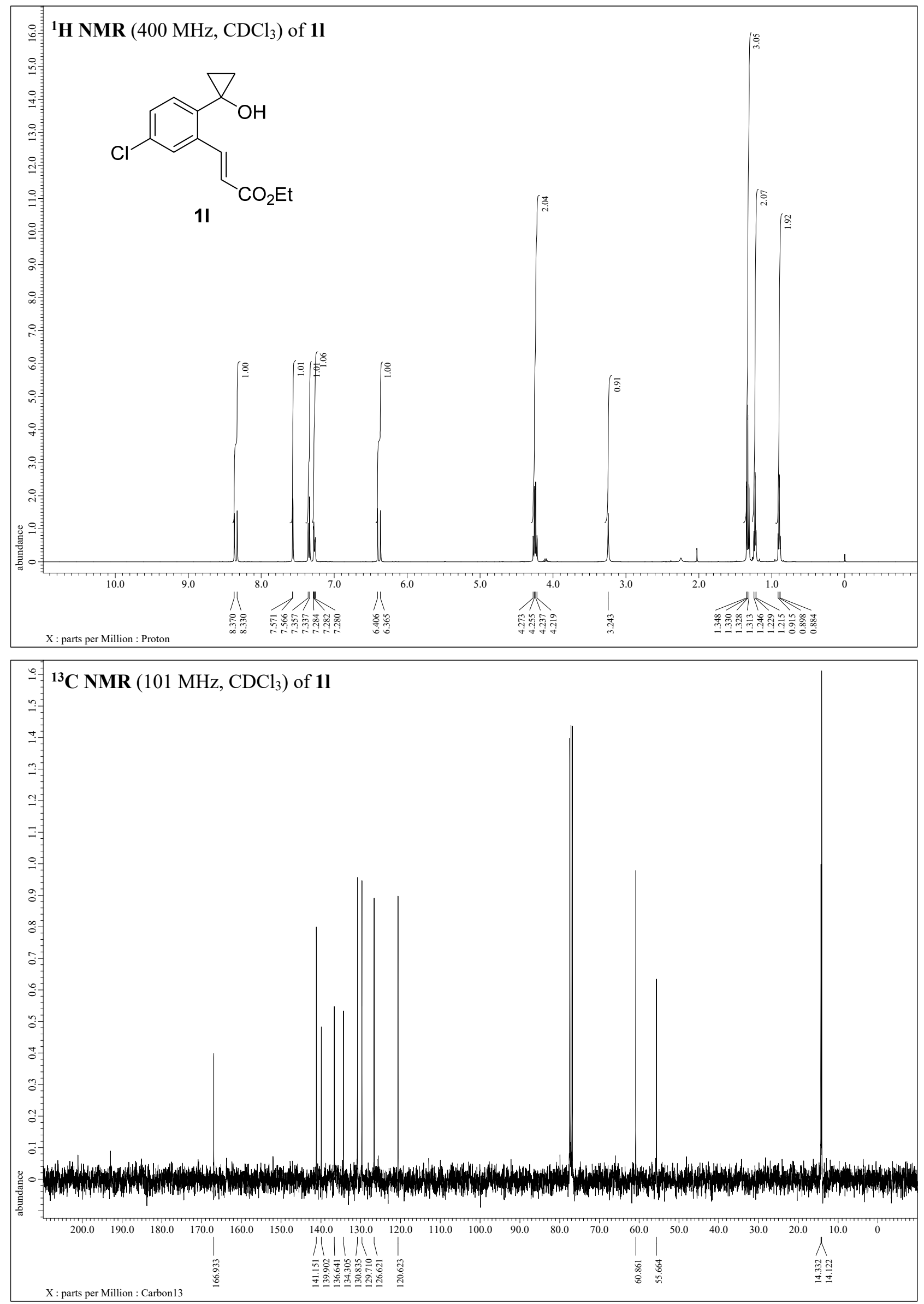

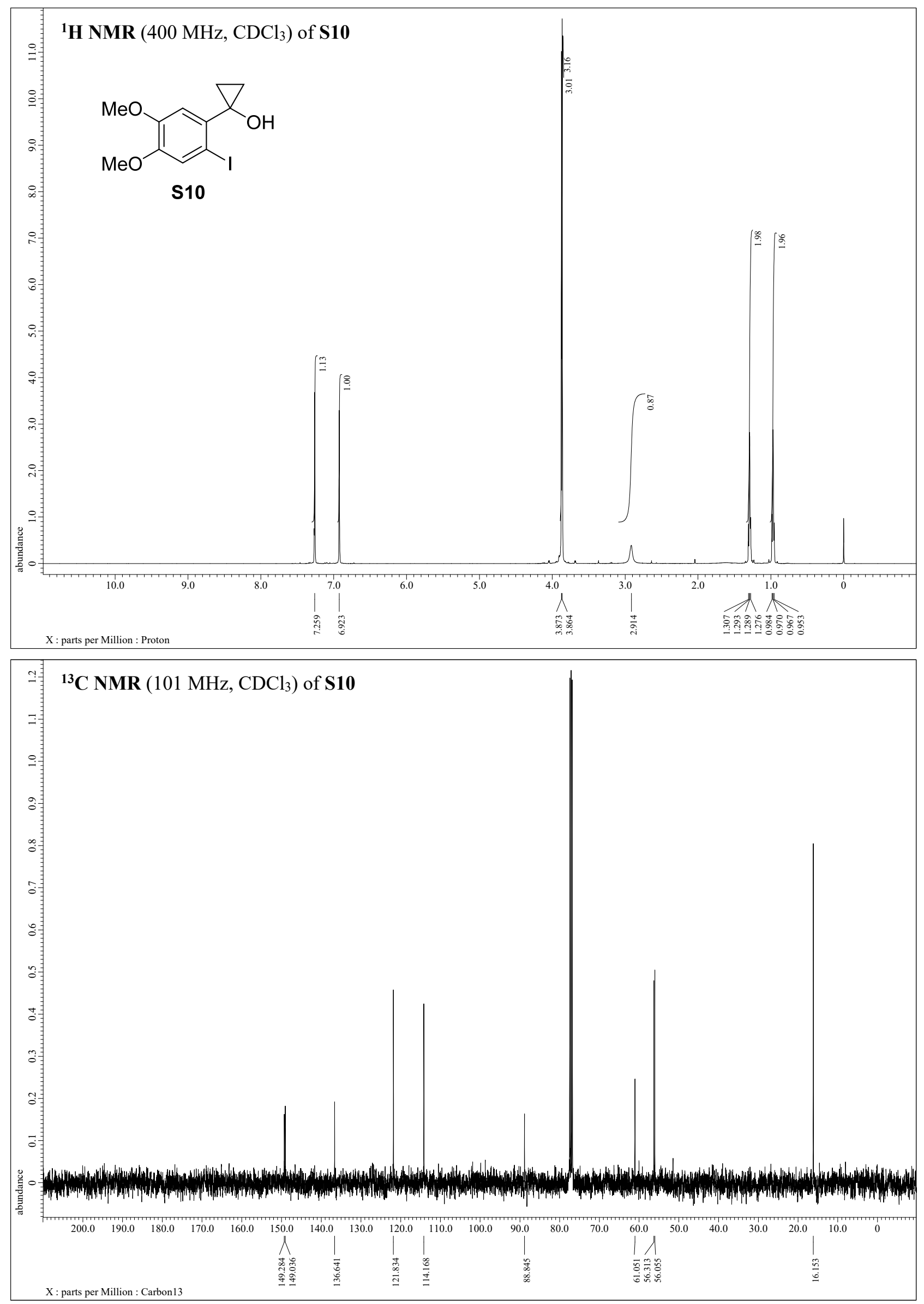

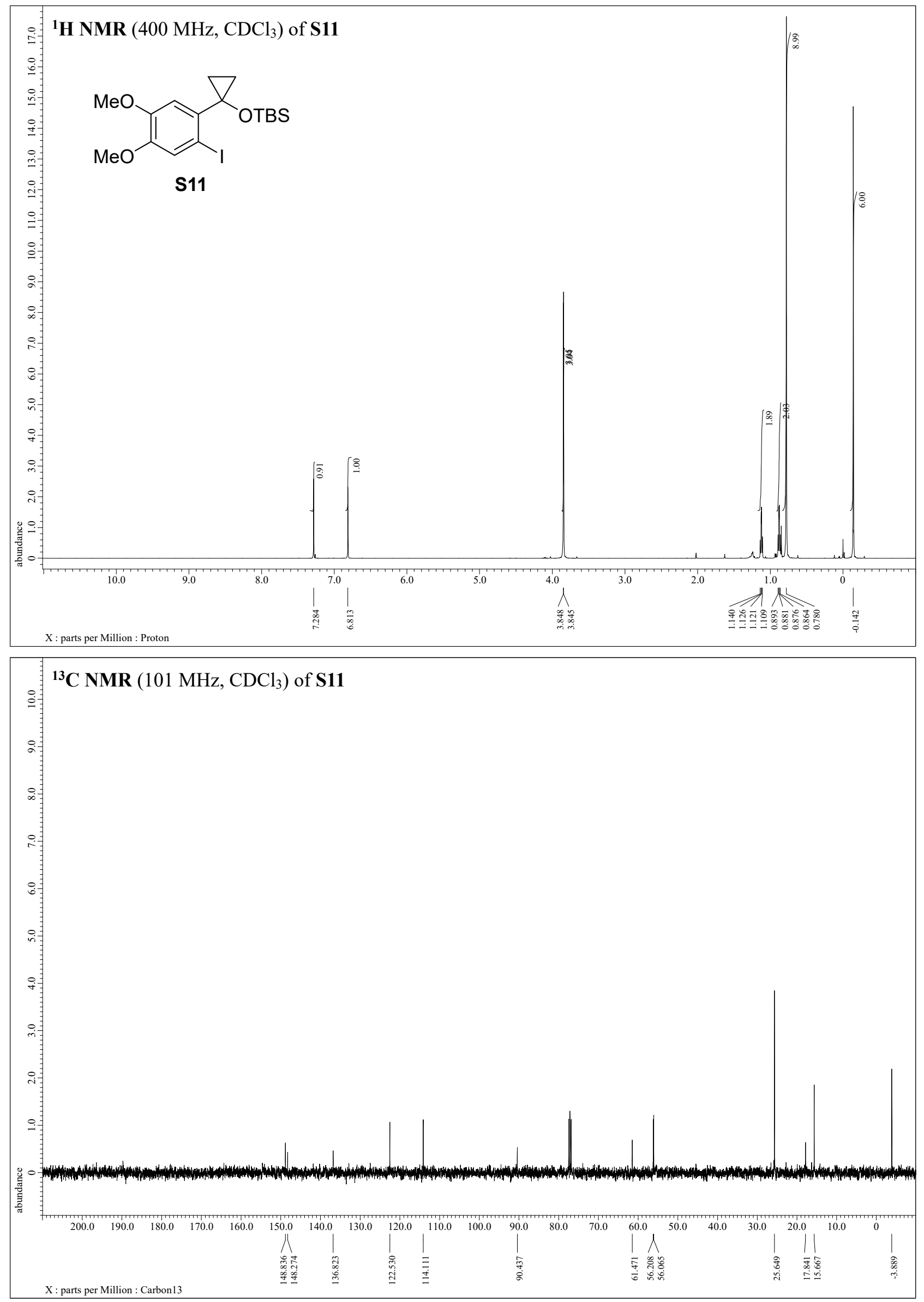

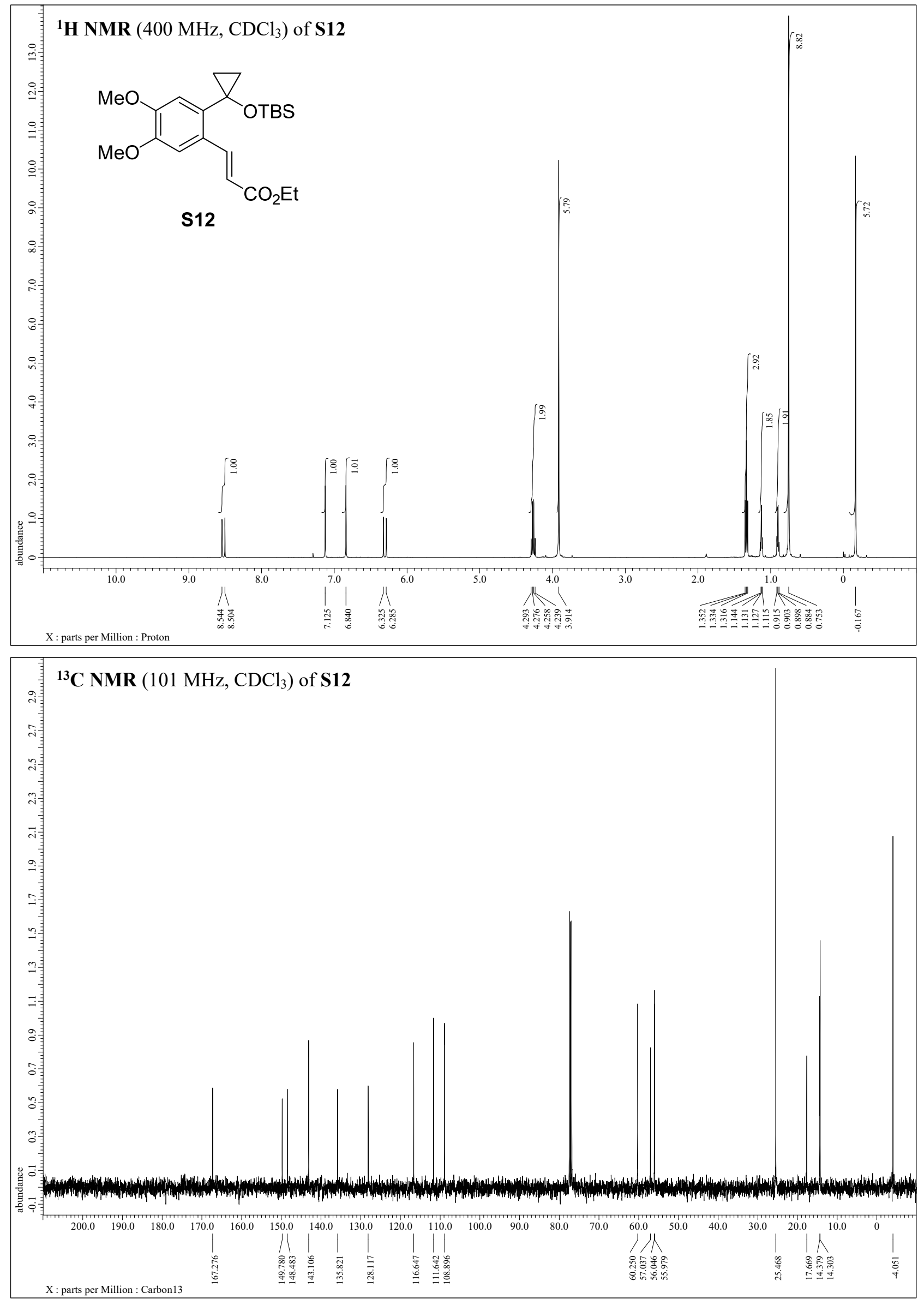

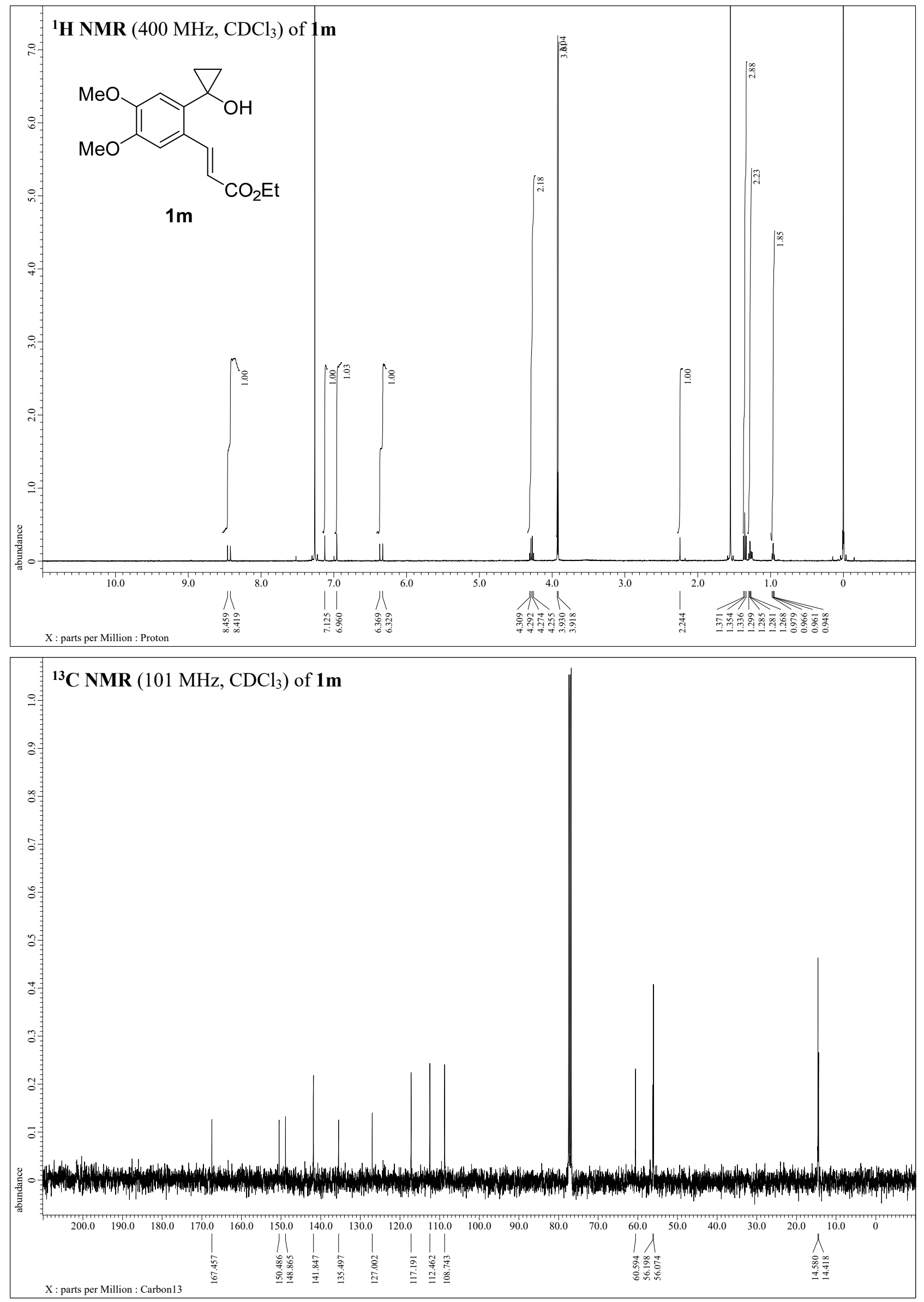

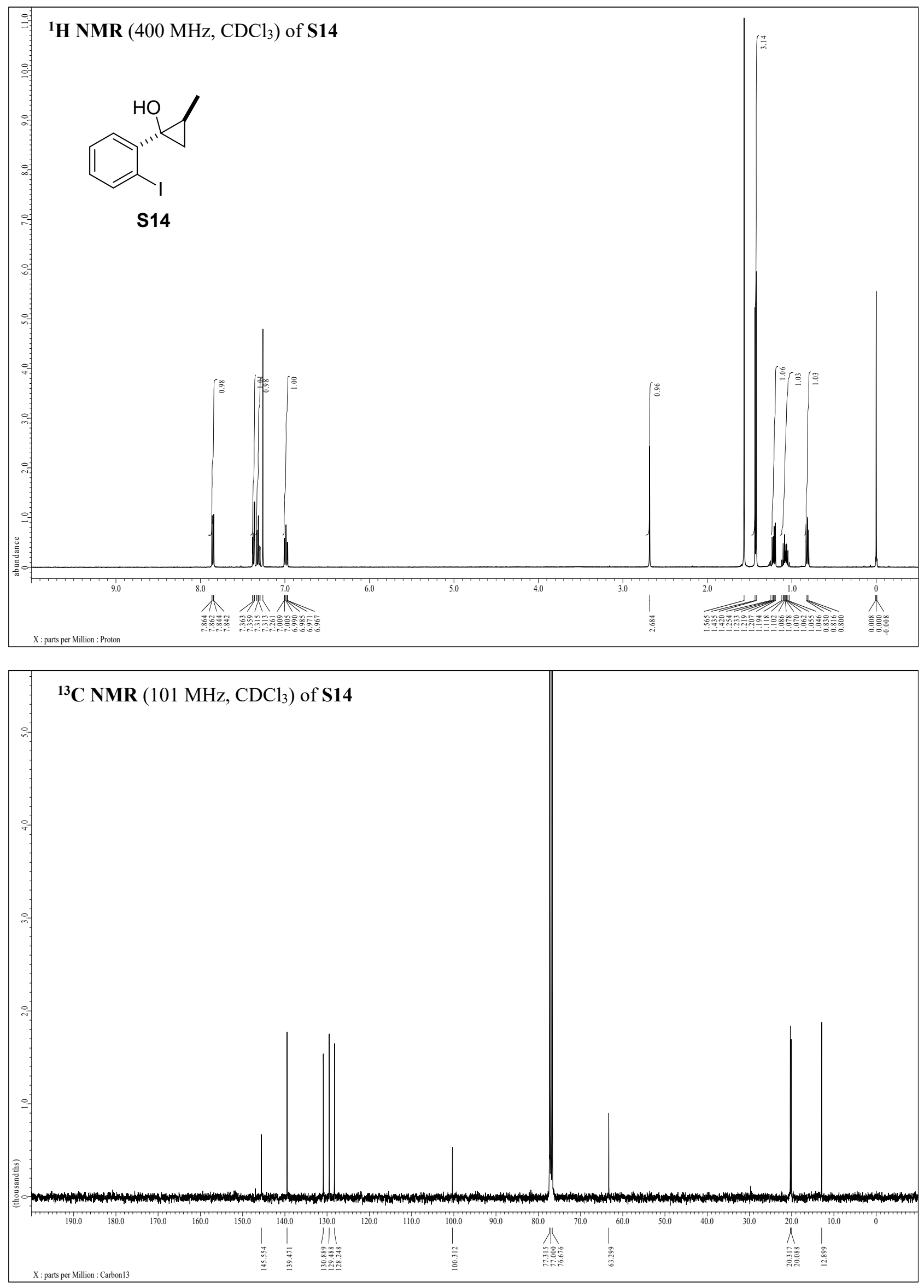

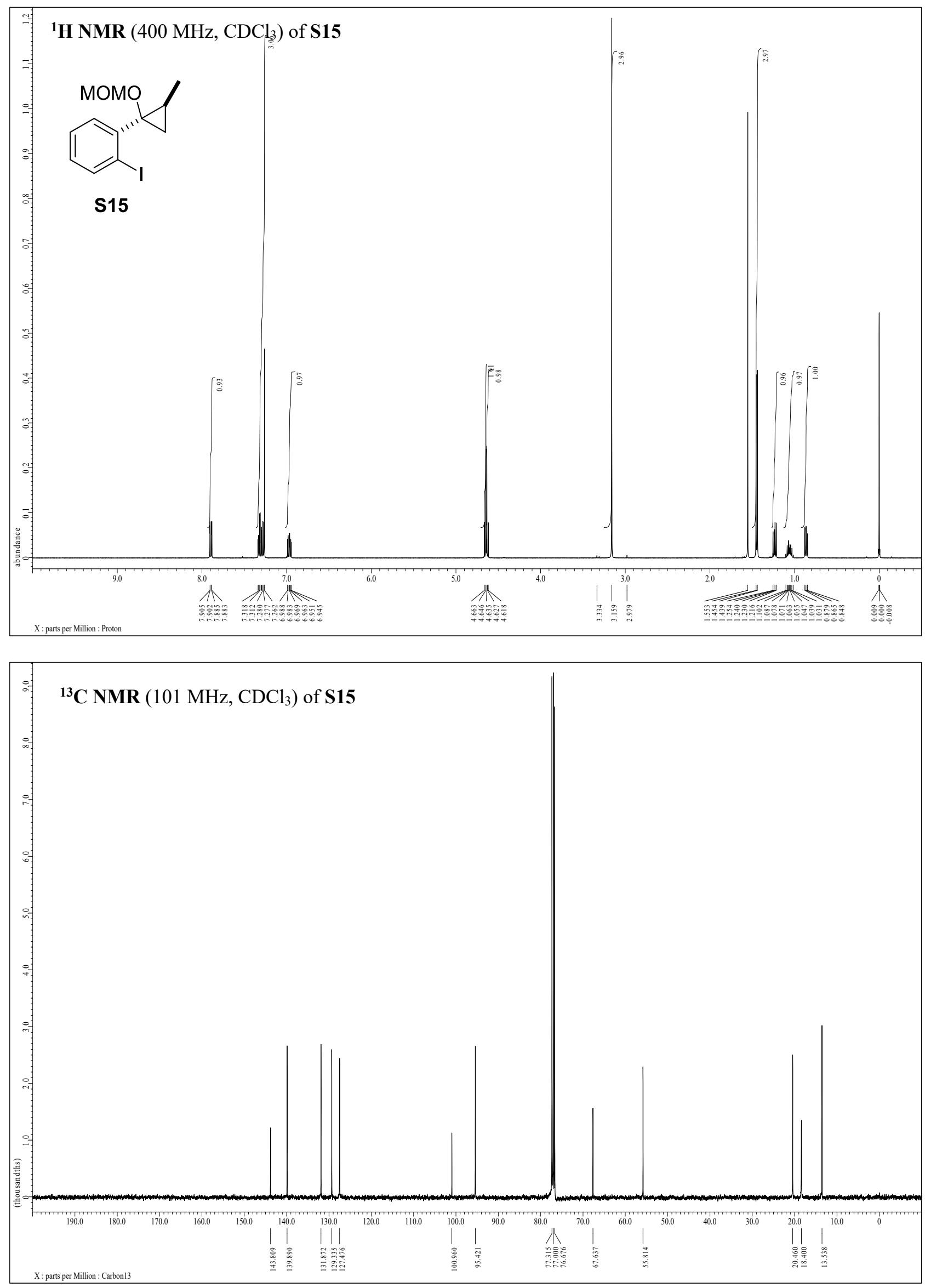
Supporting Information

598
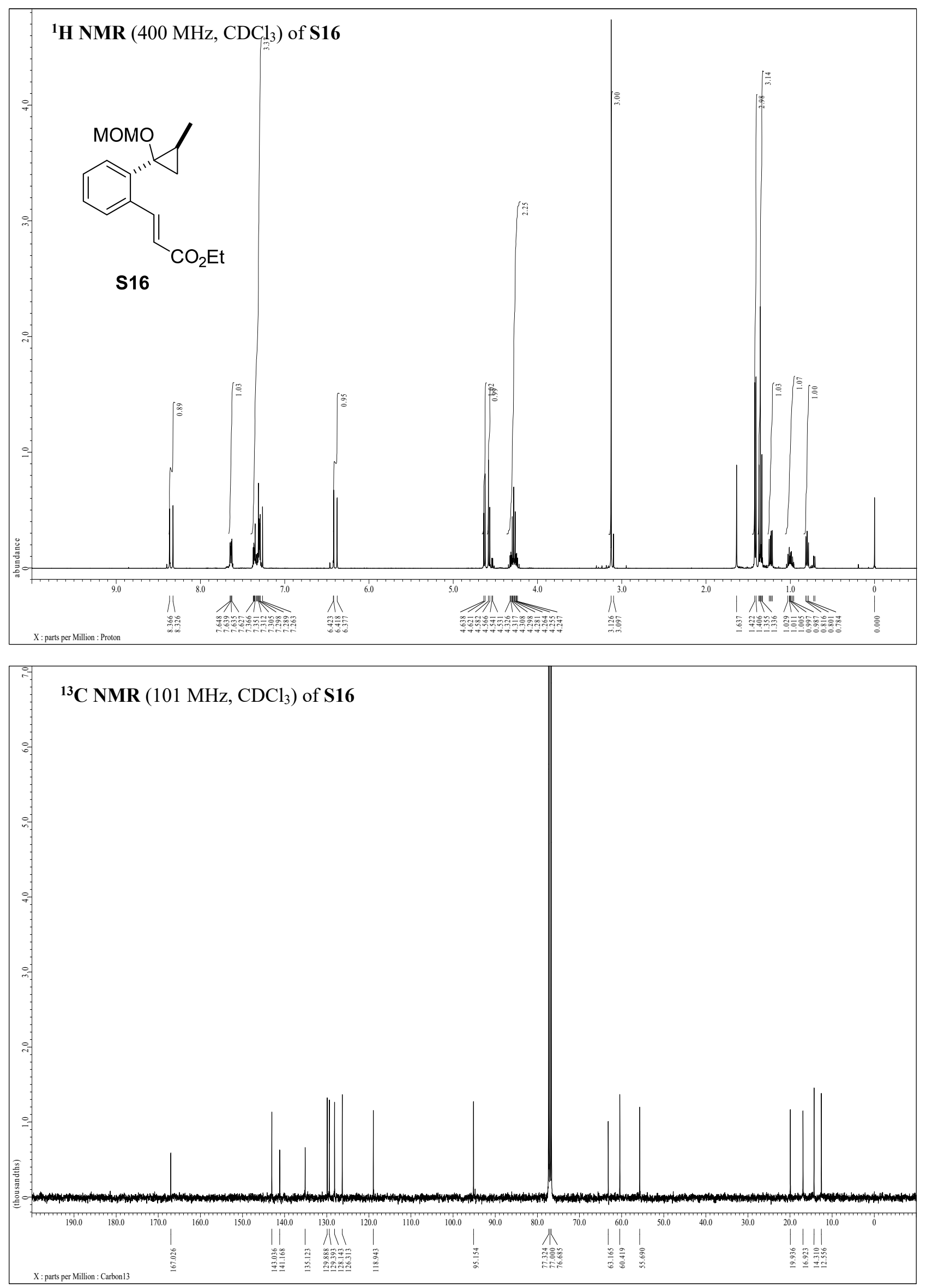
Supporting Information

S99
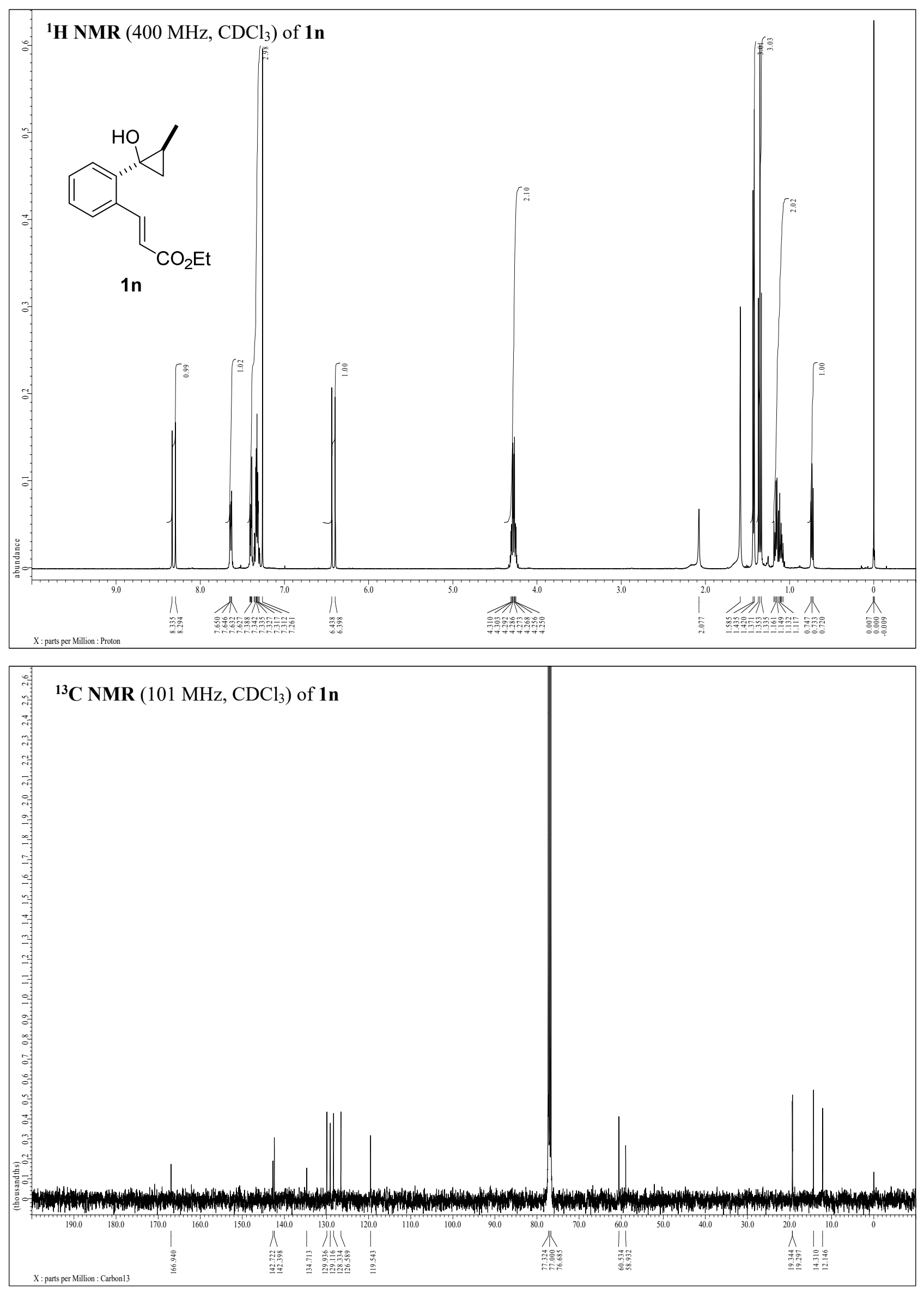

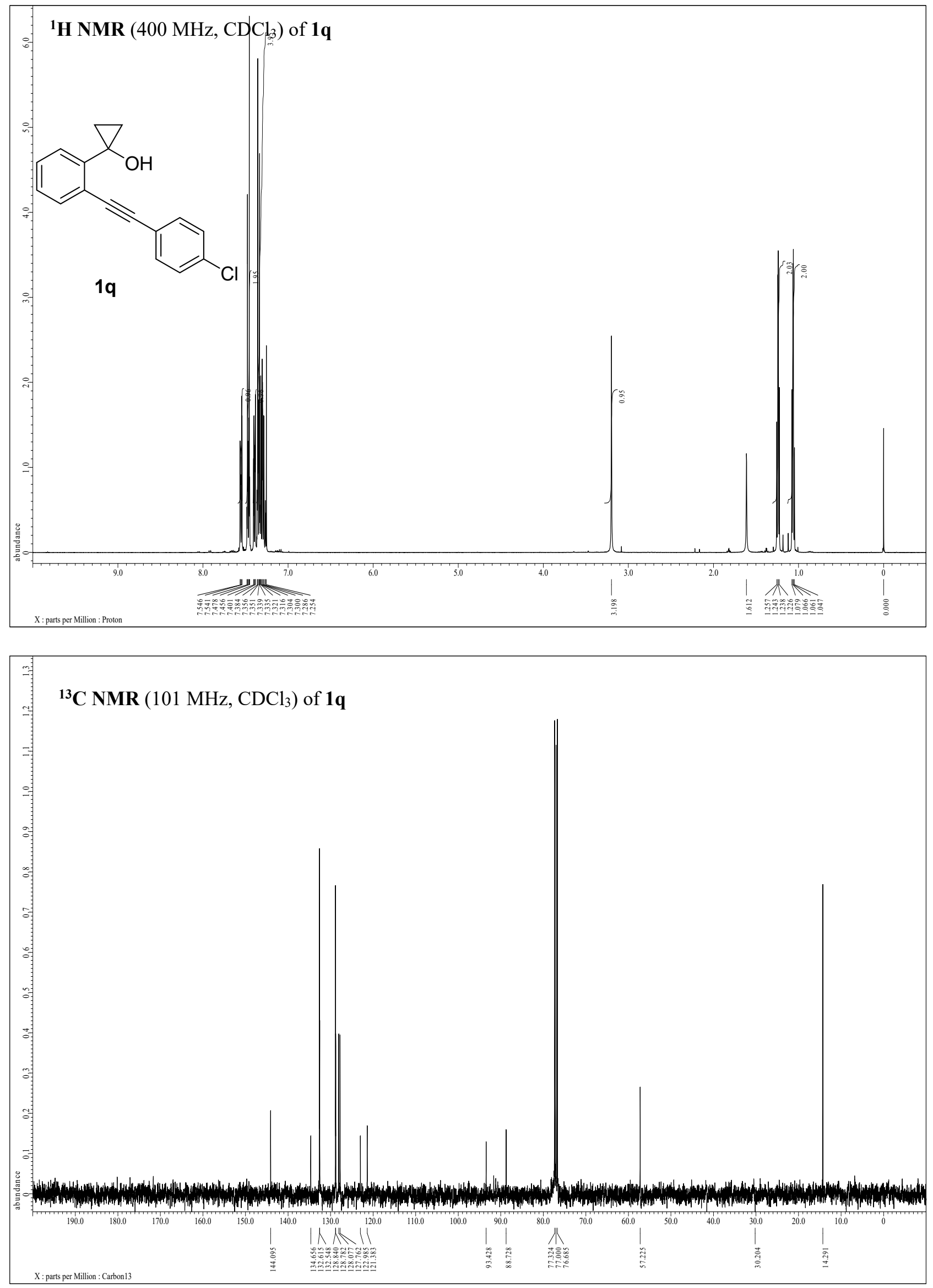

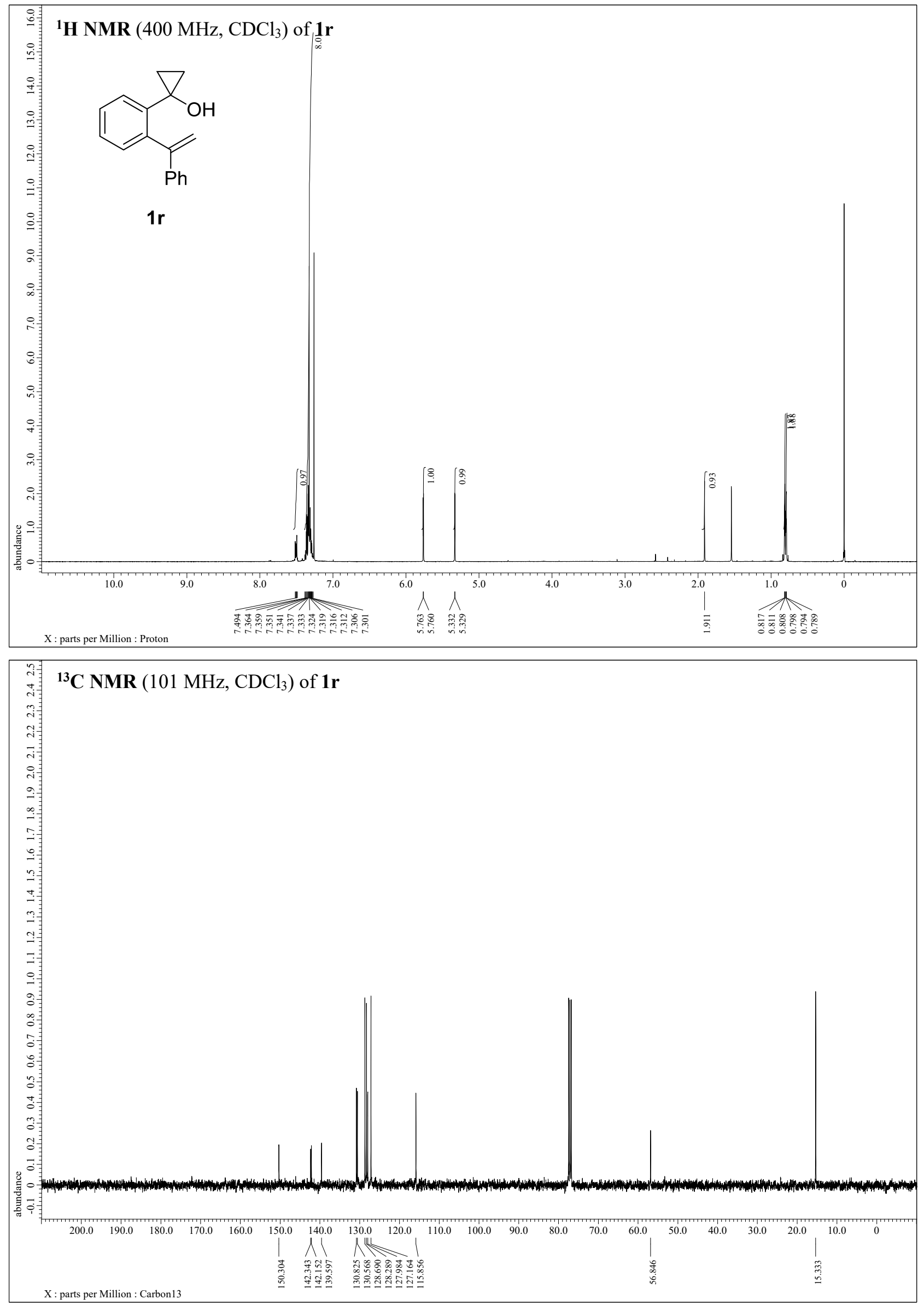

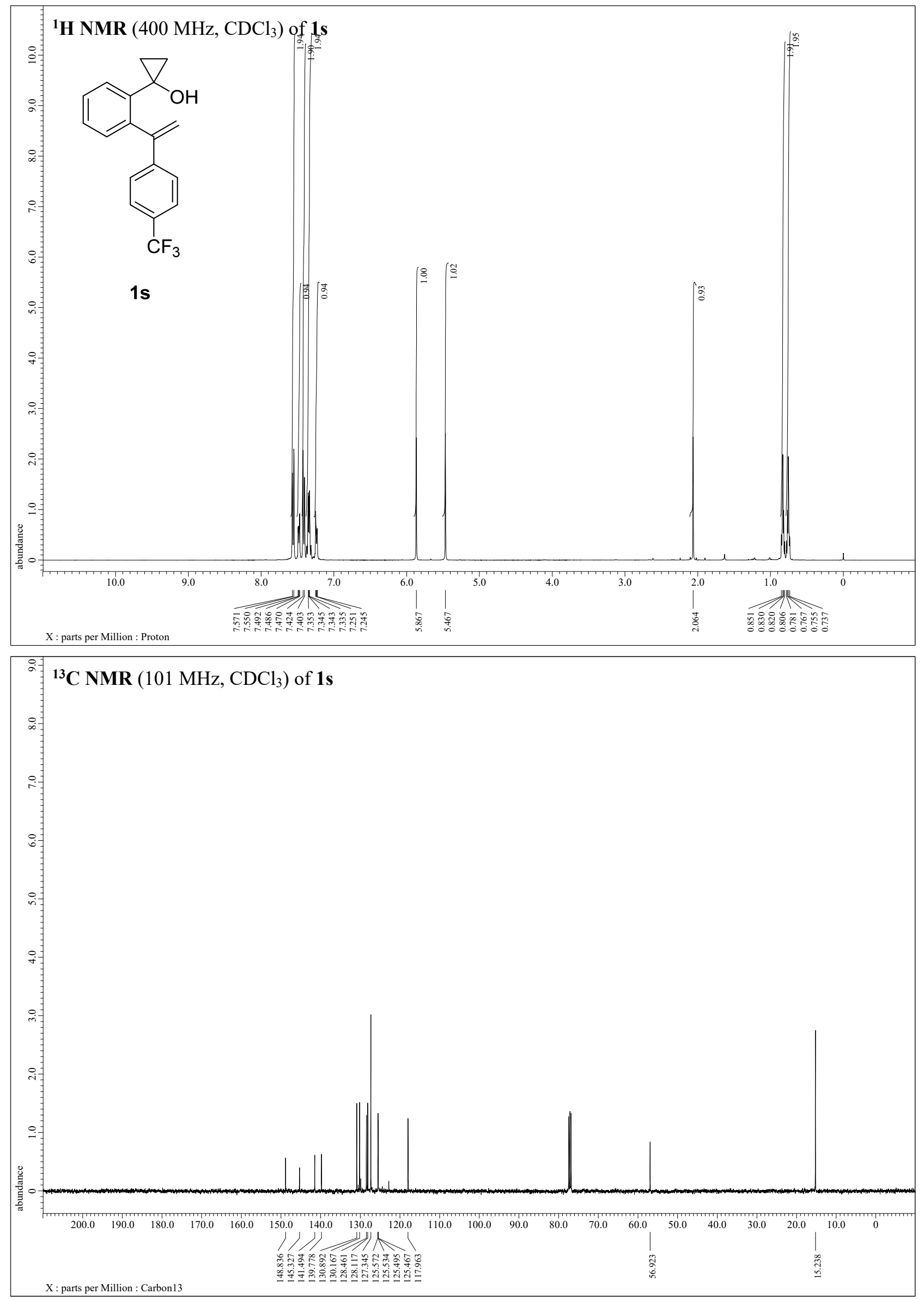

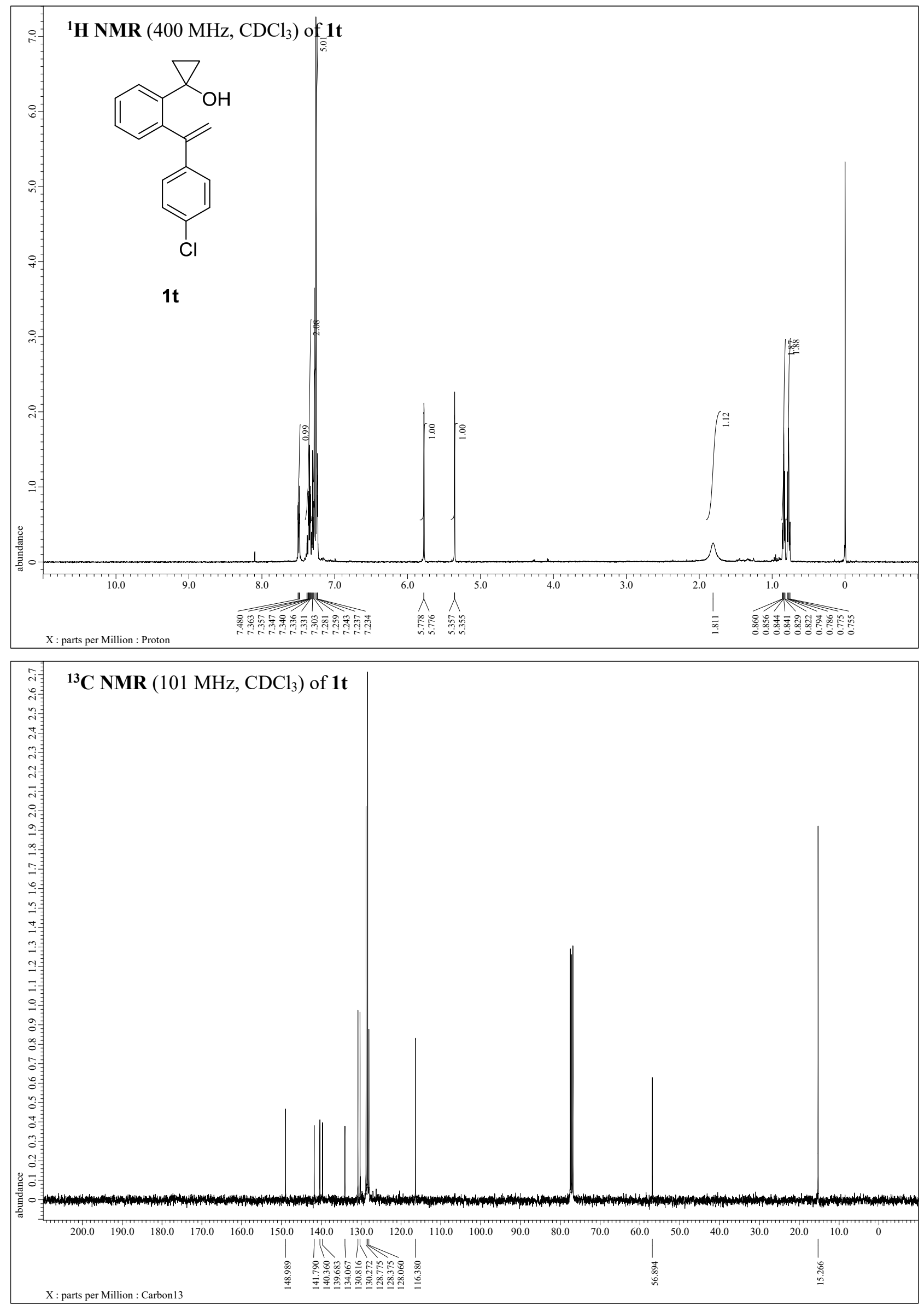

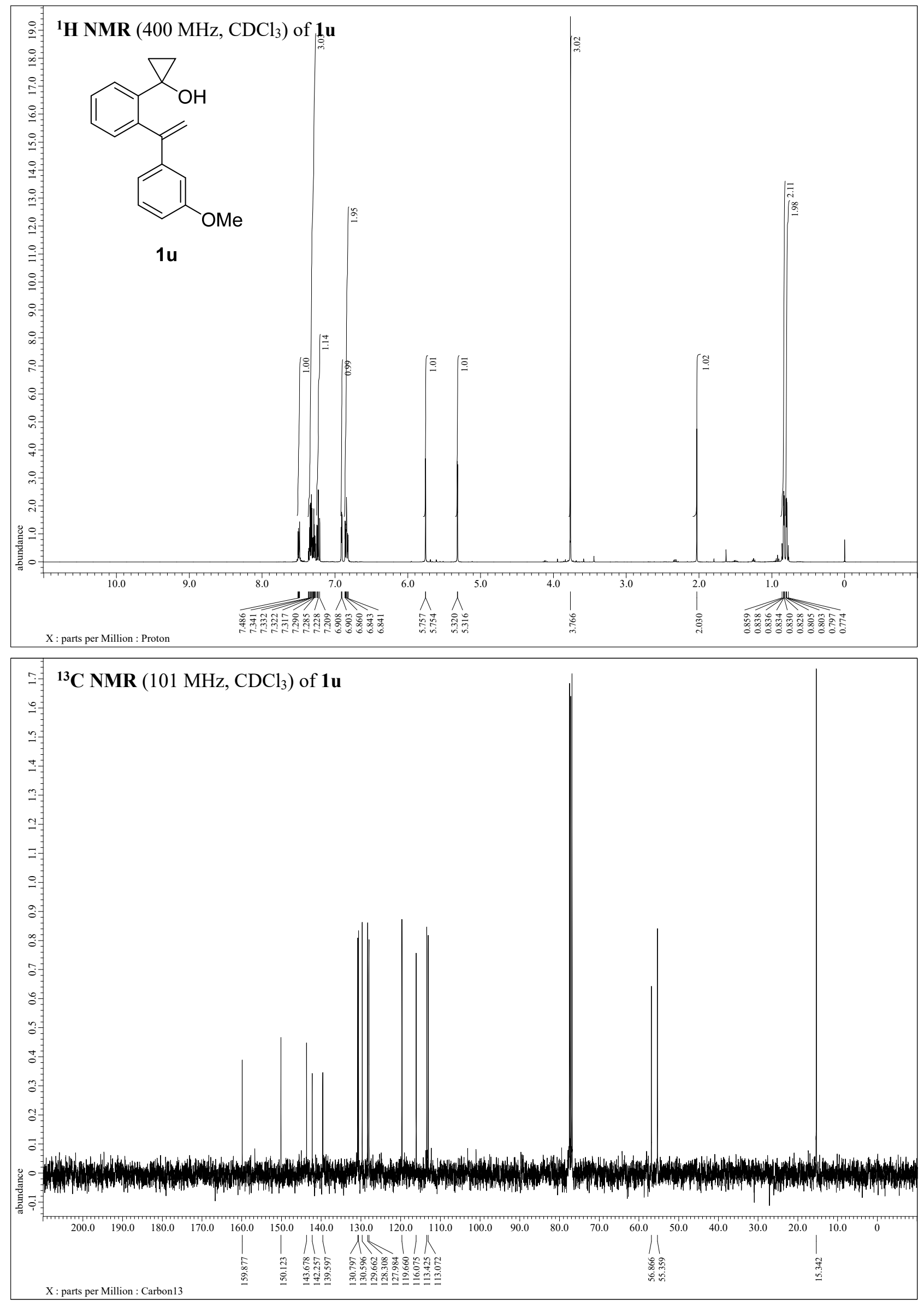

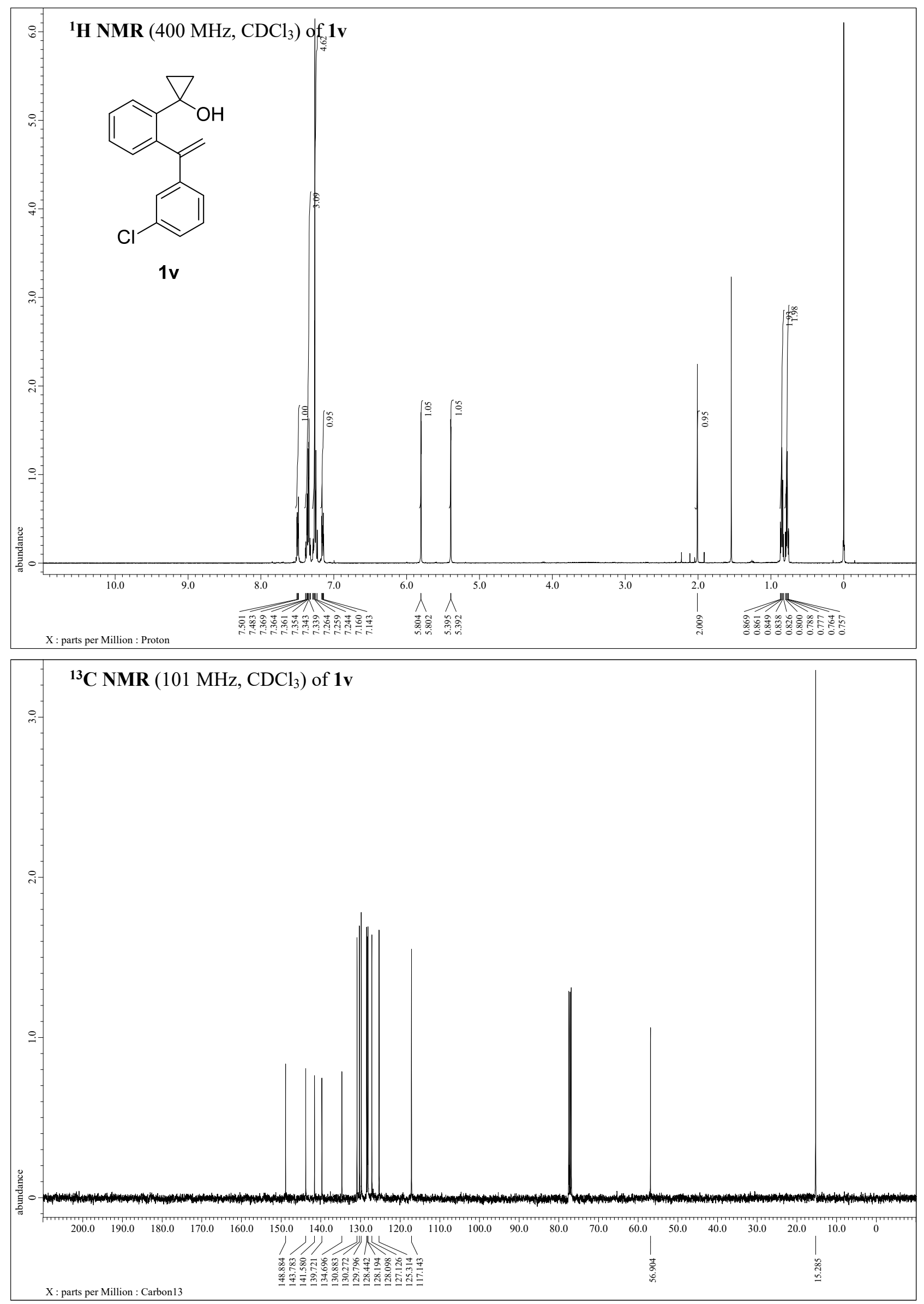

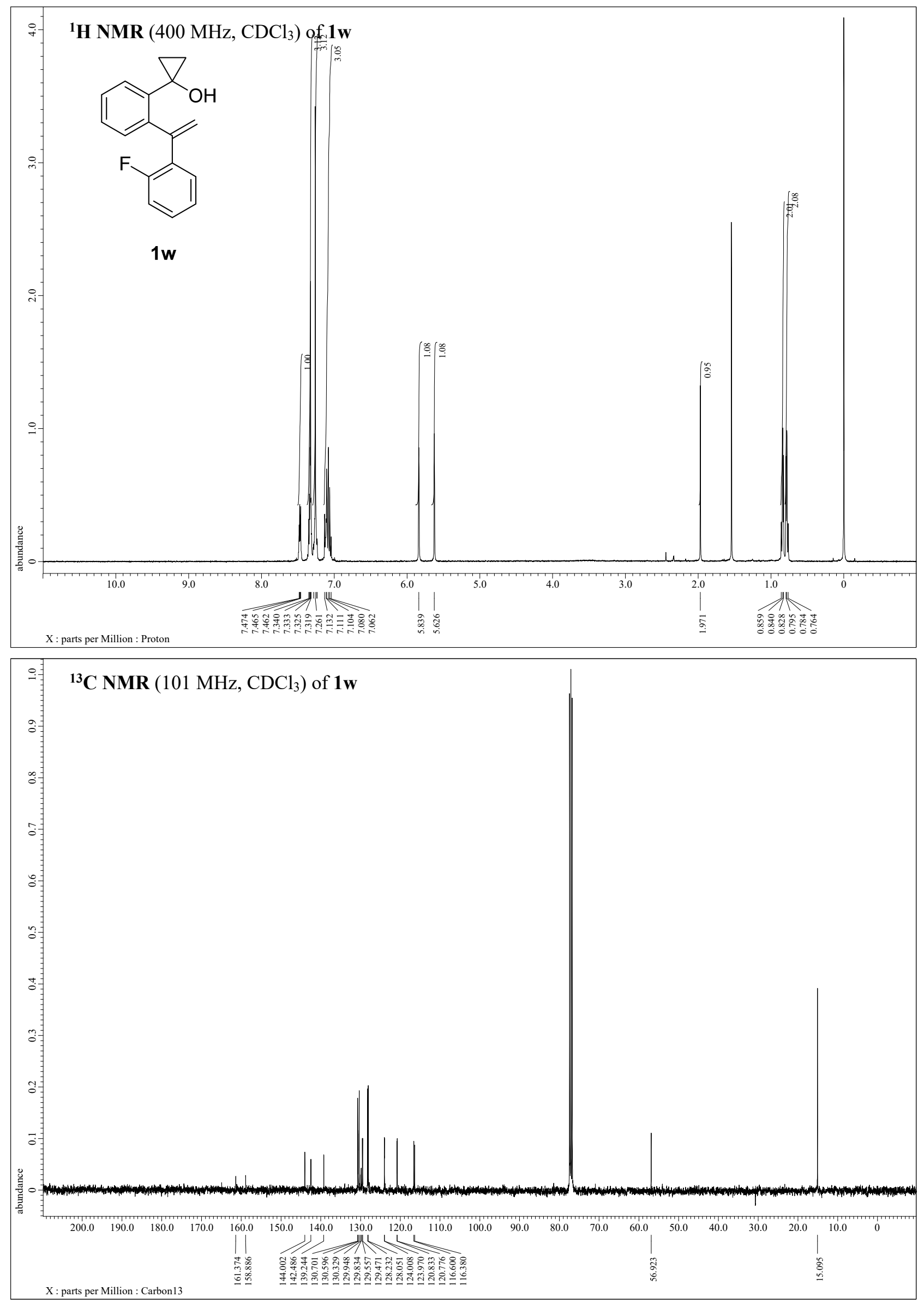

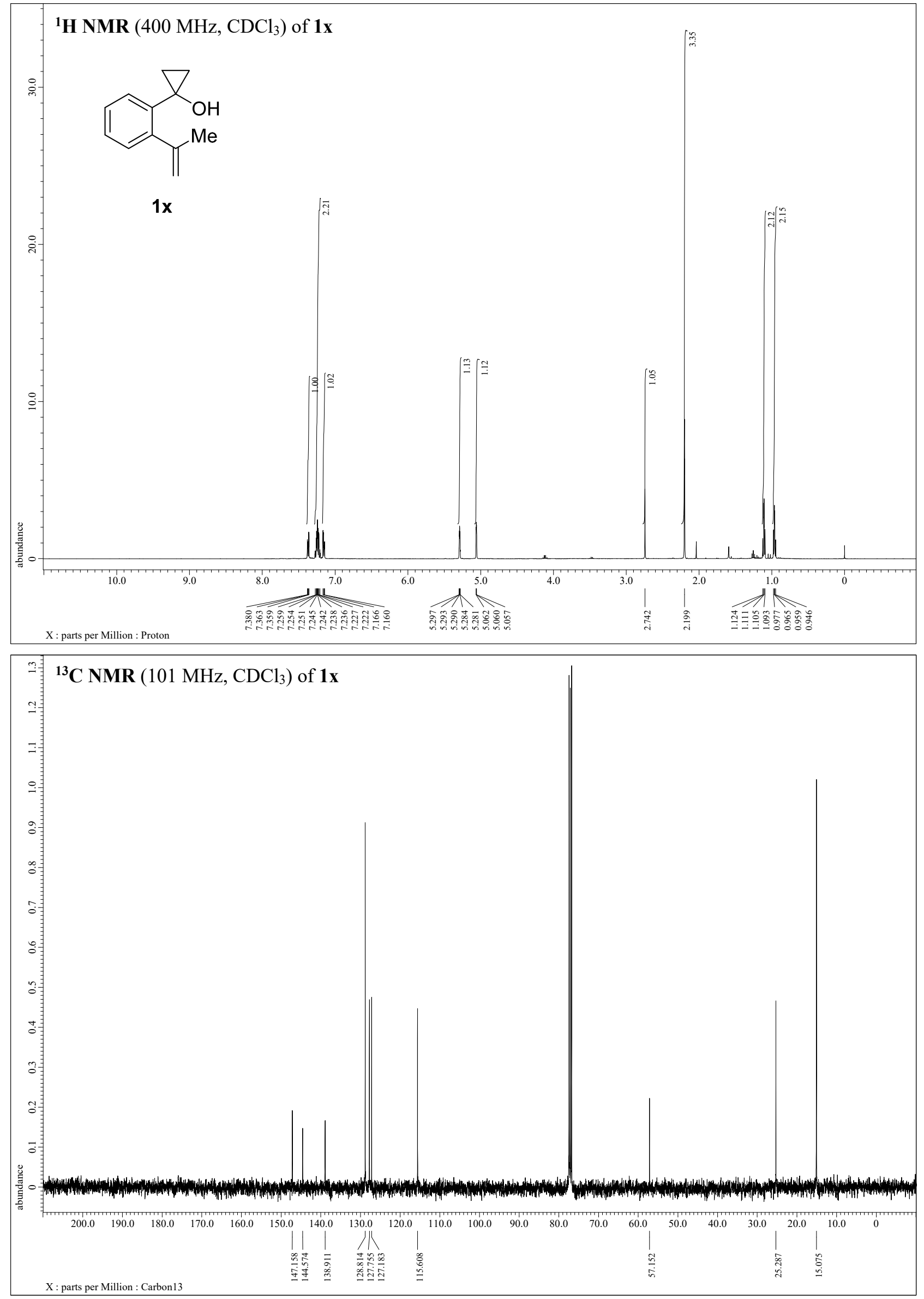

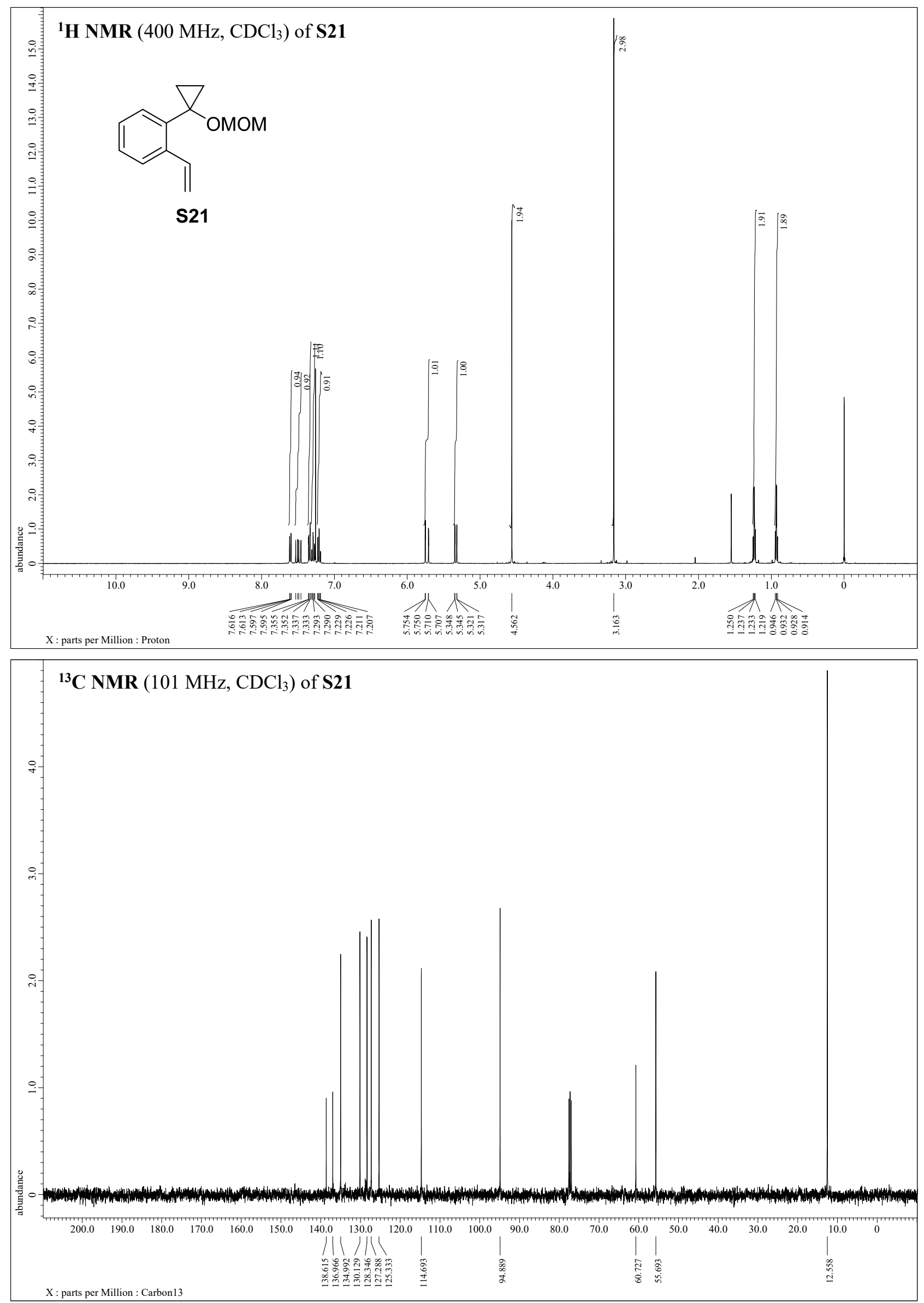

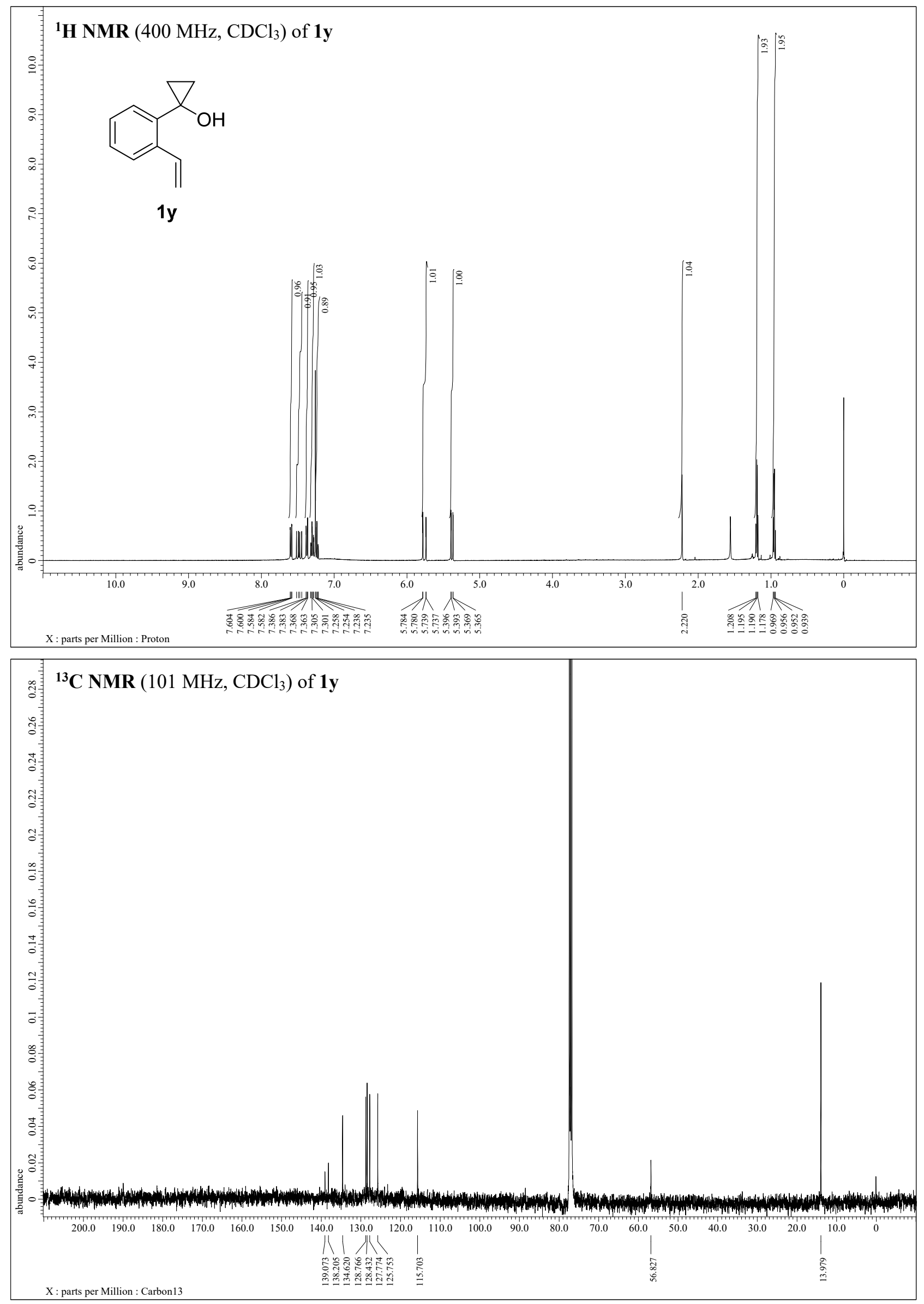

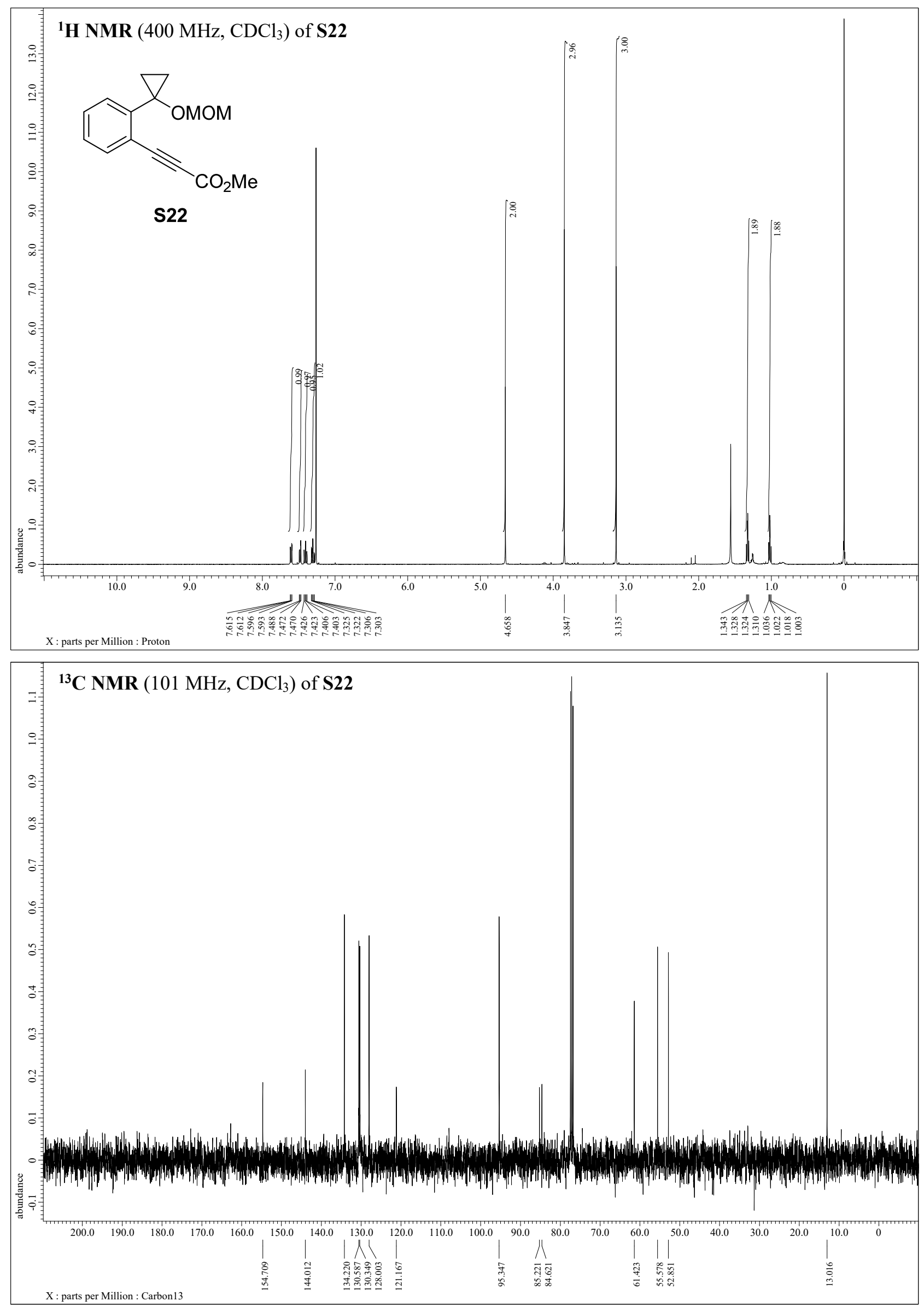

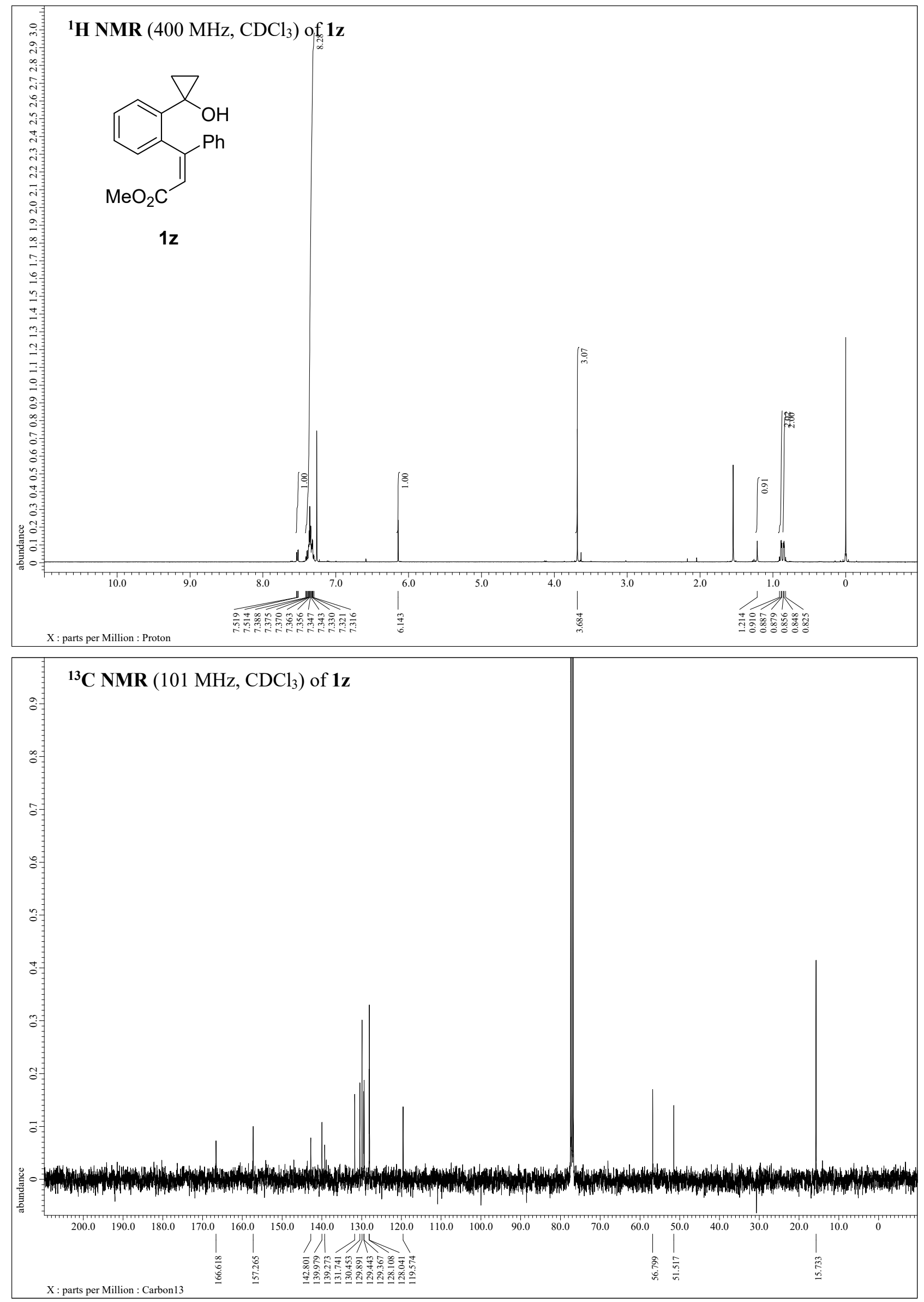

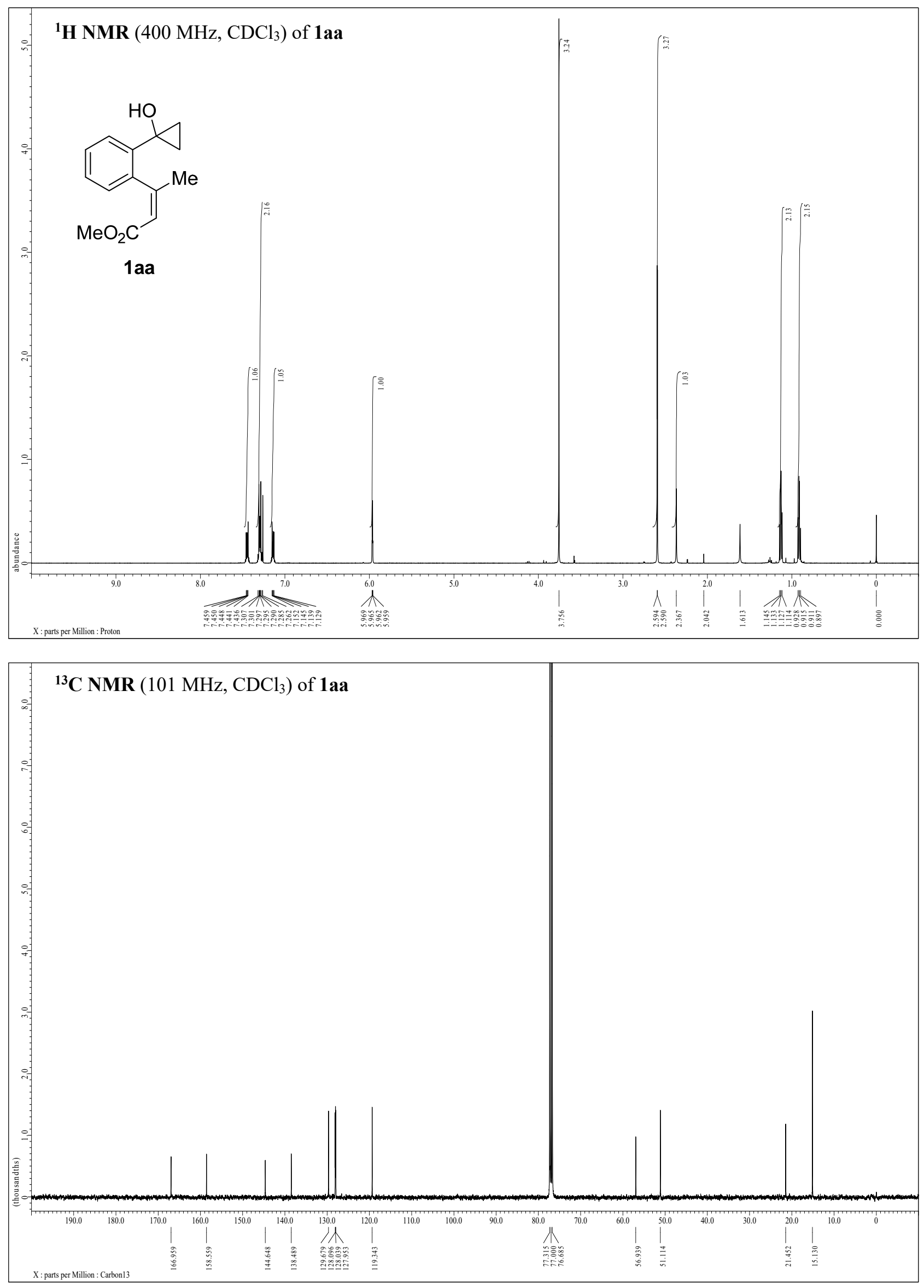

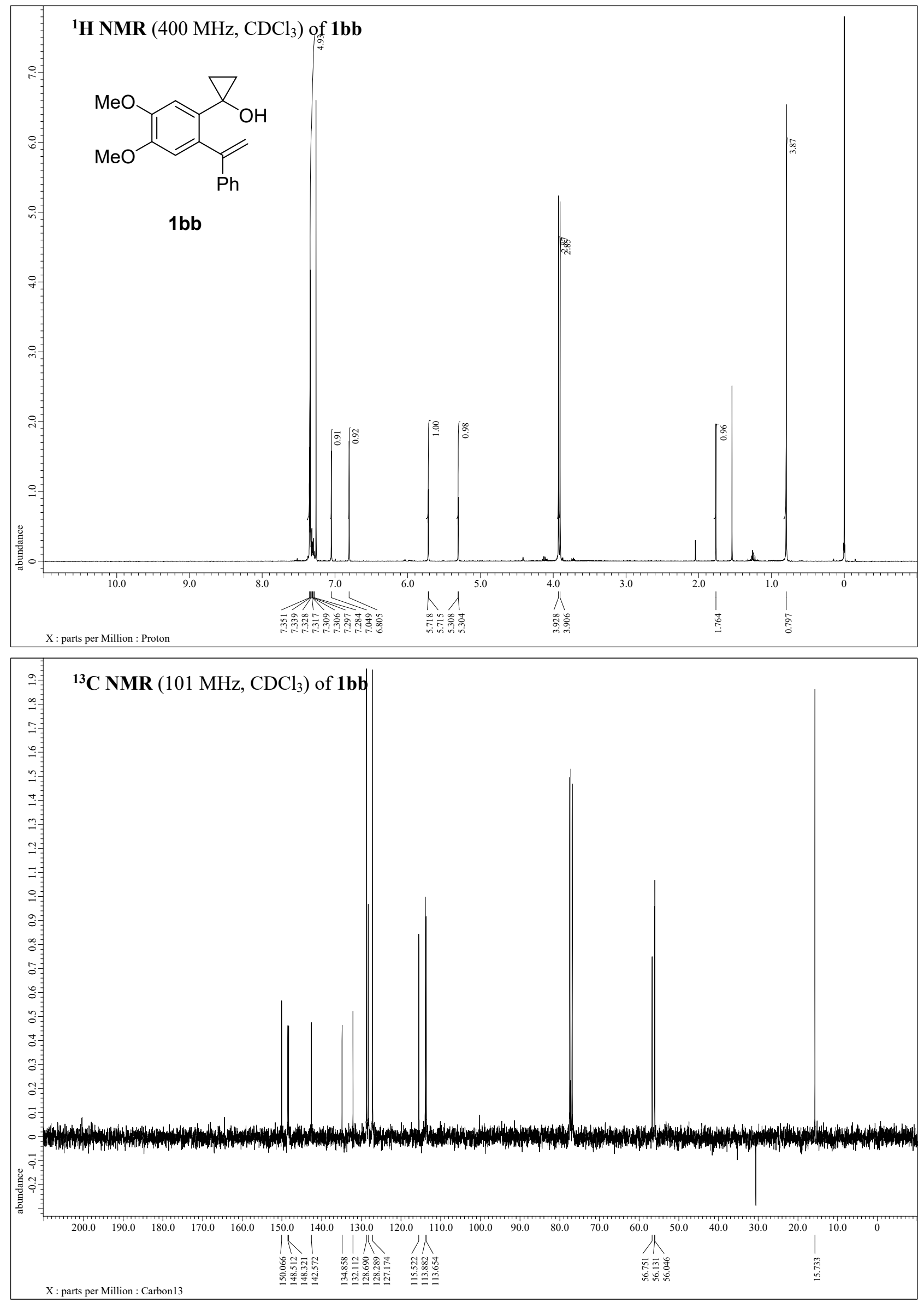

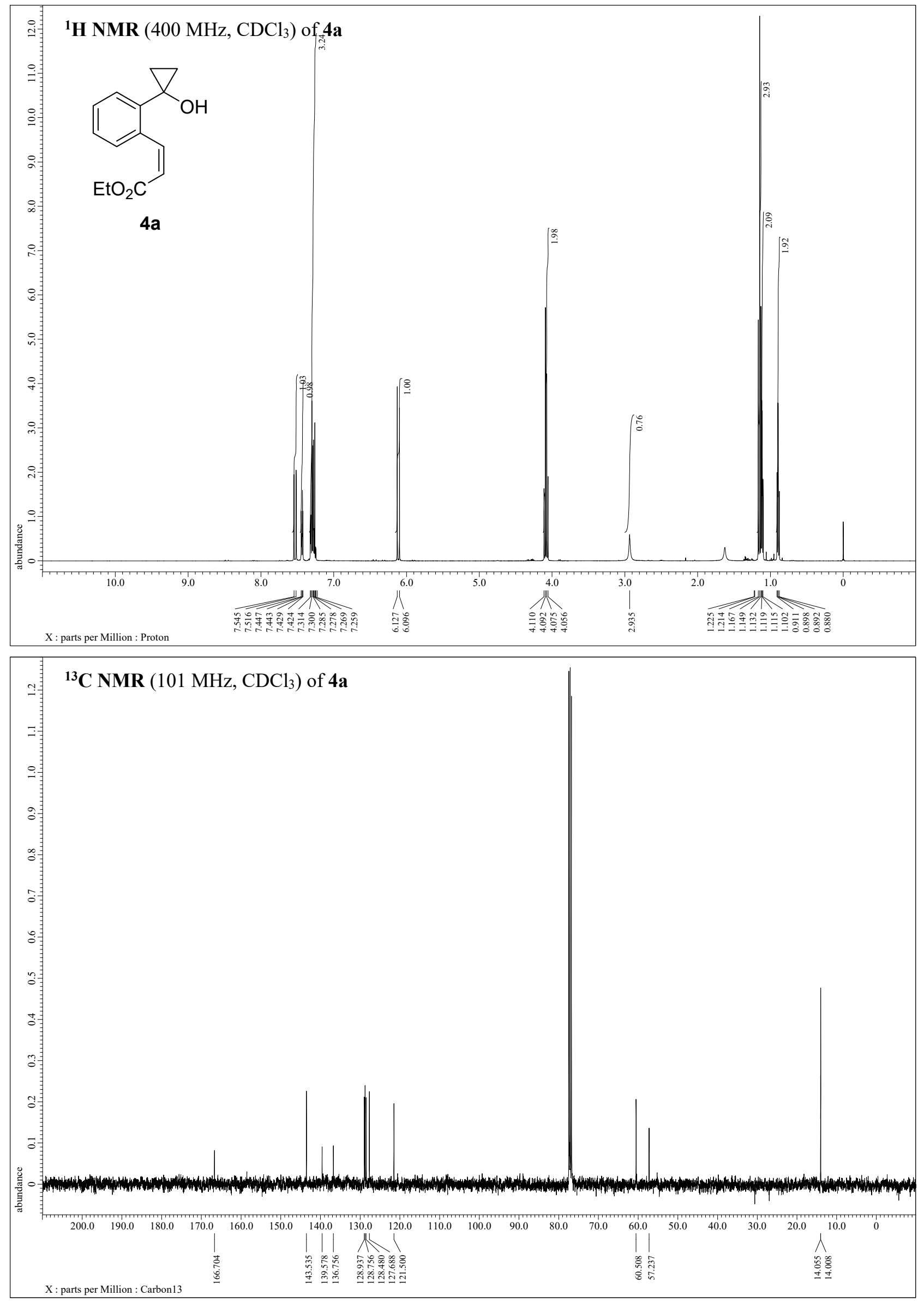

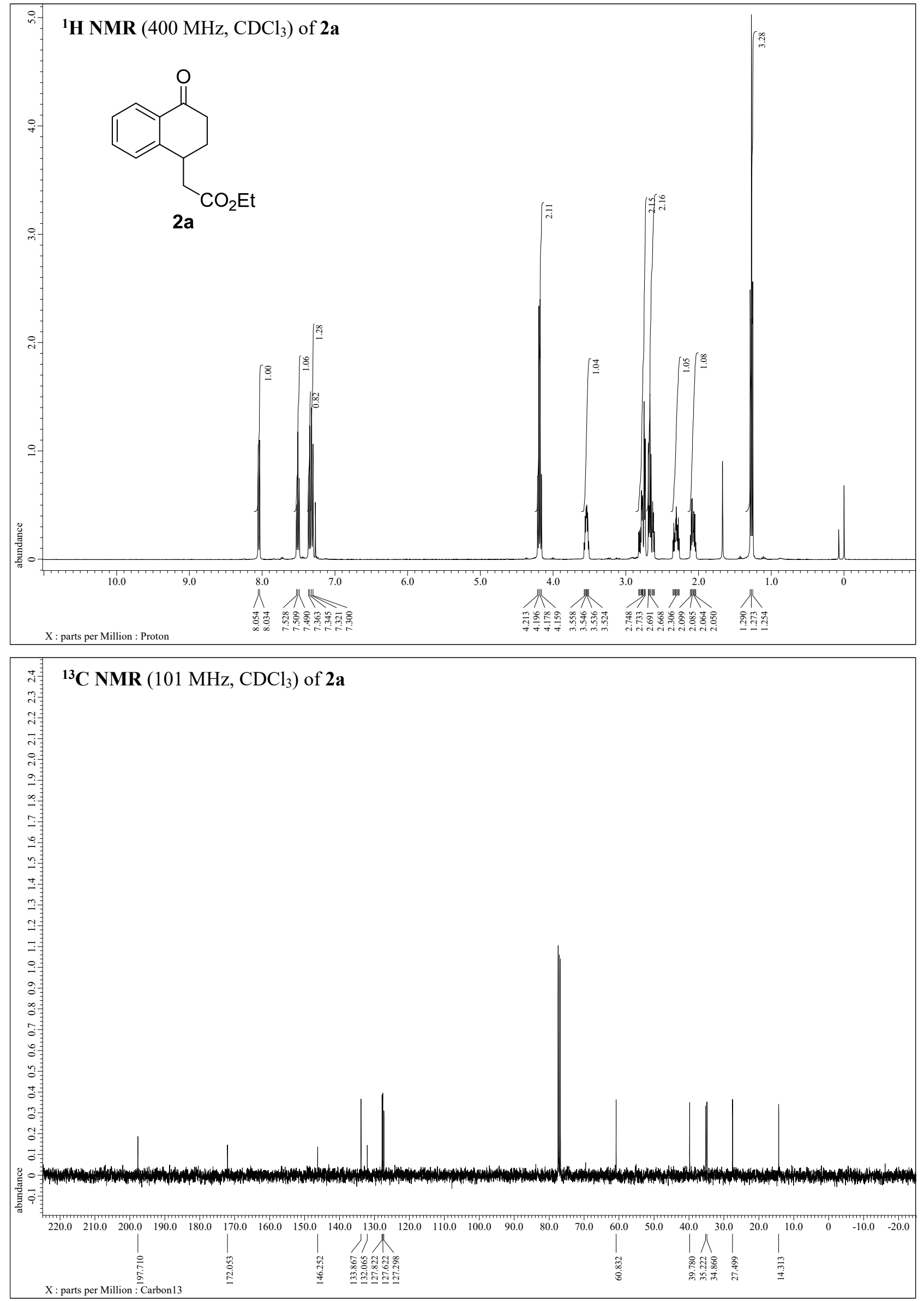

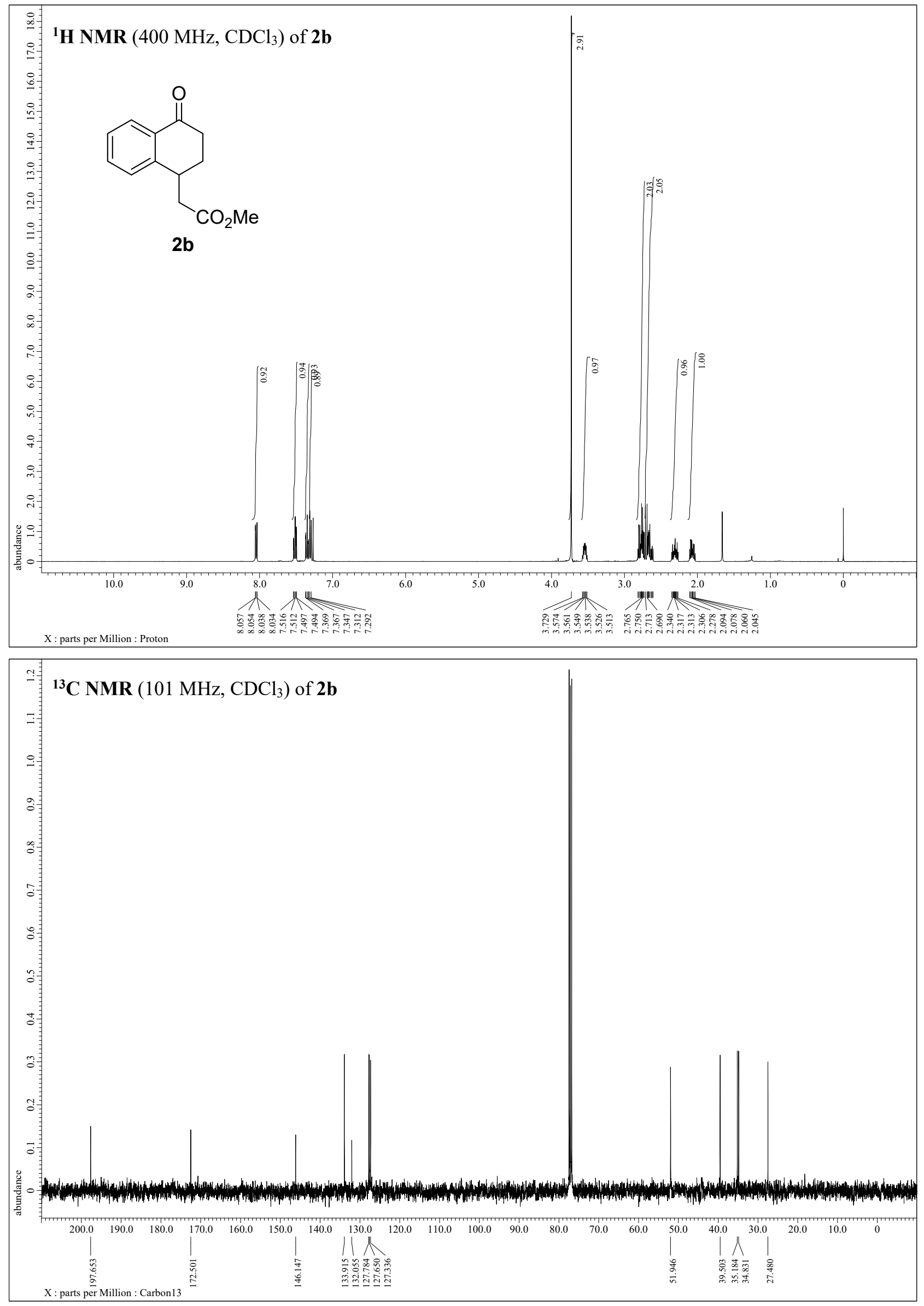

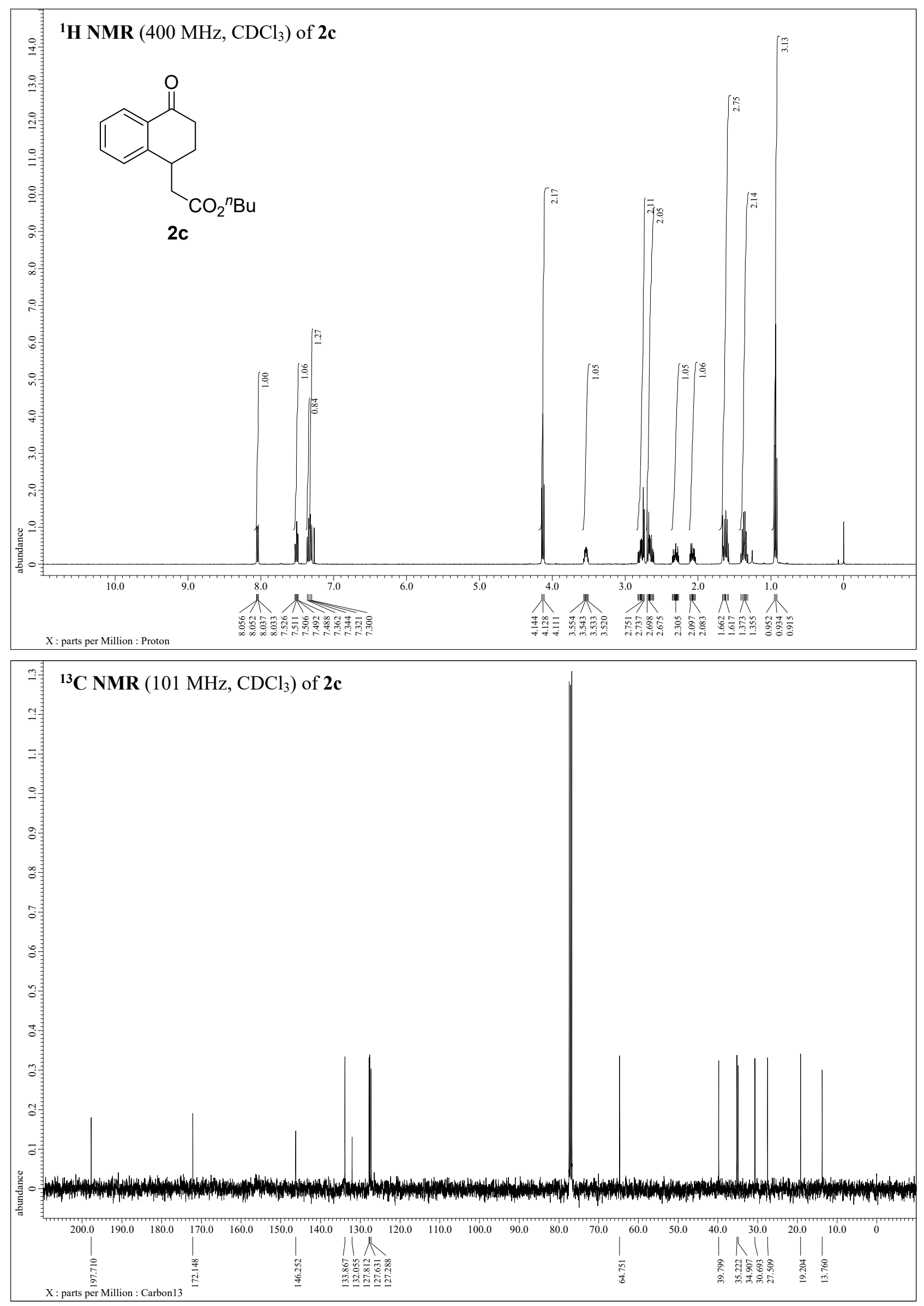

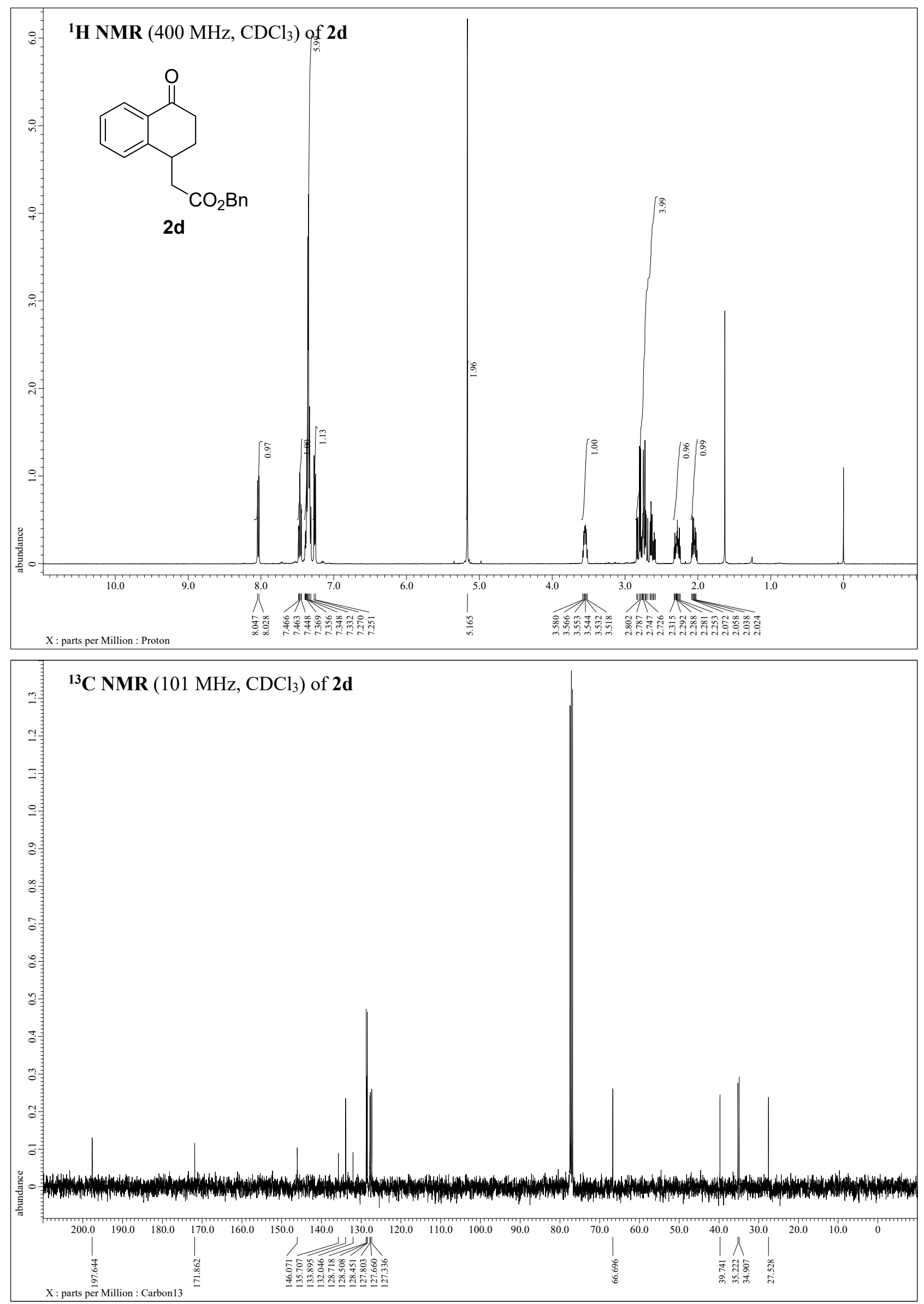

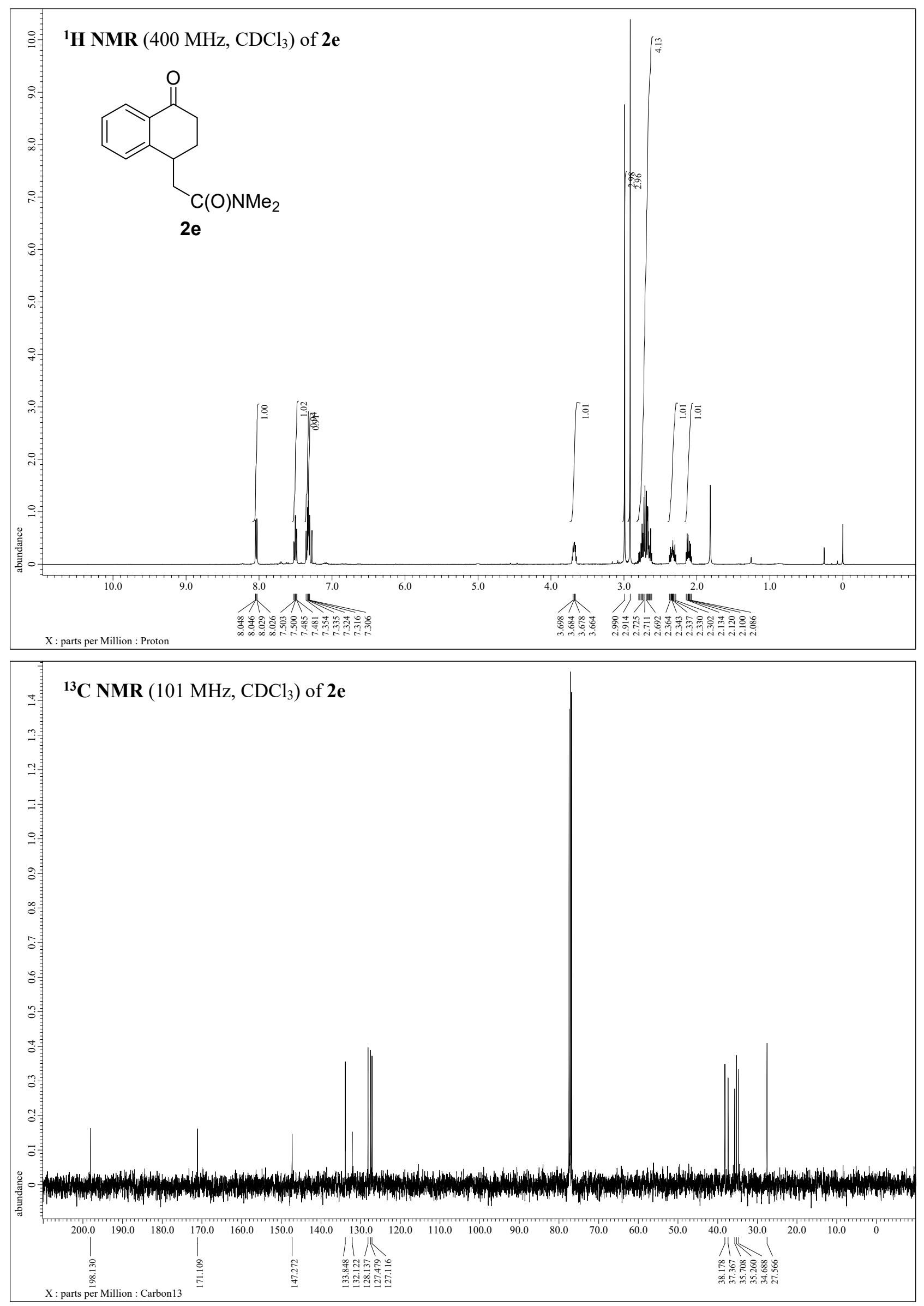

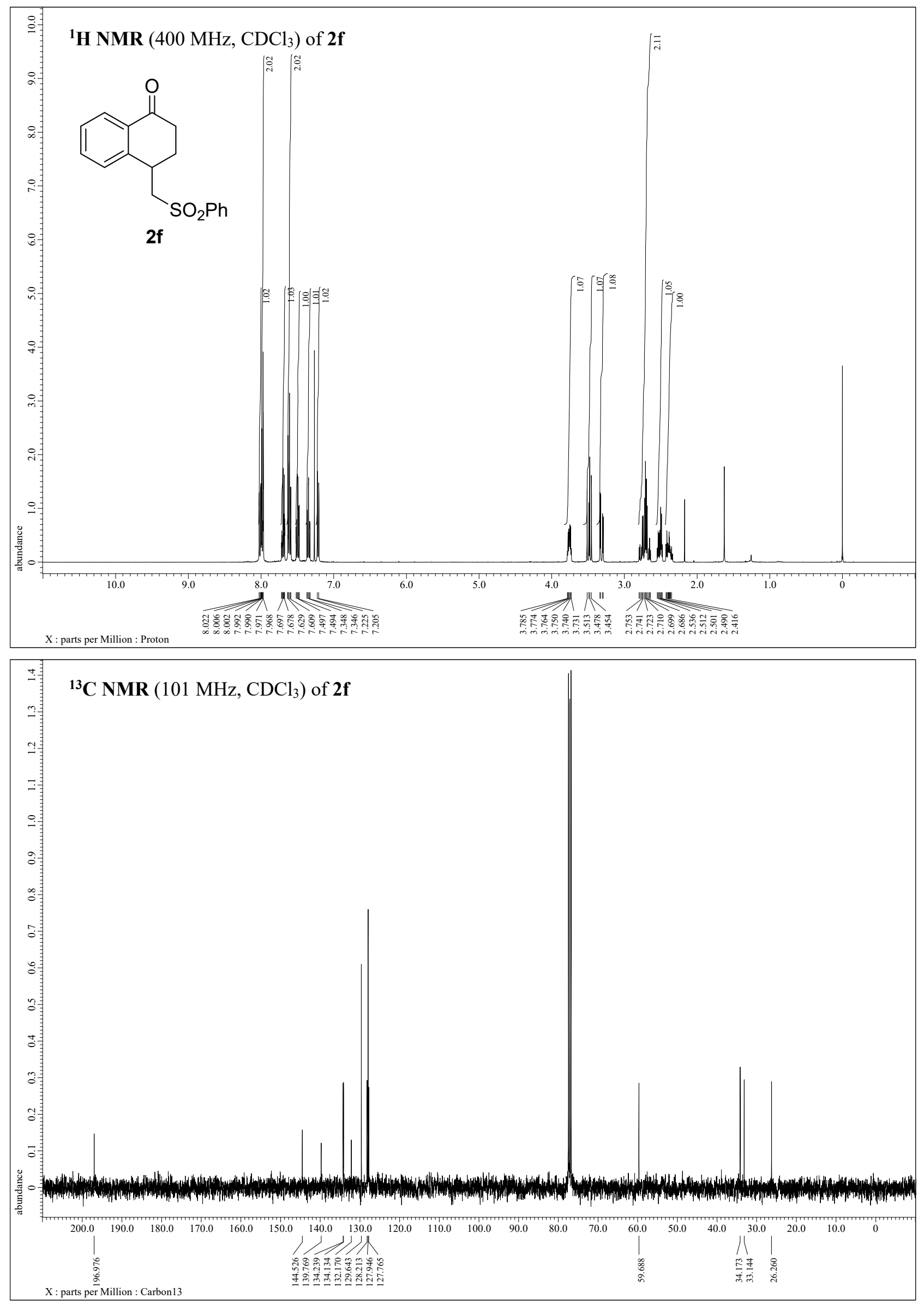

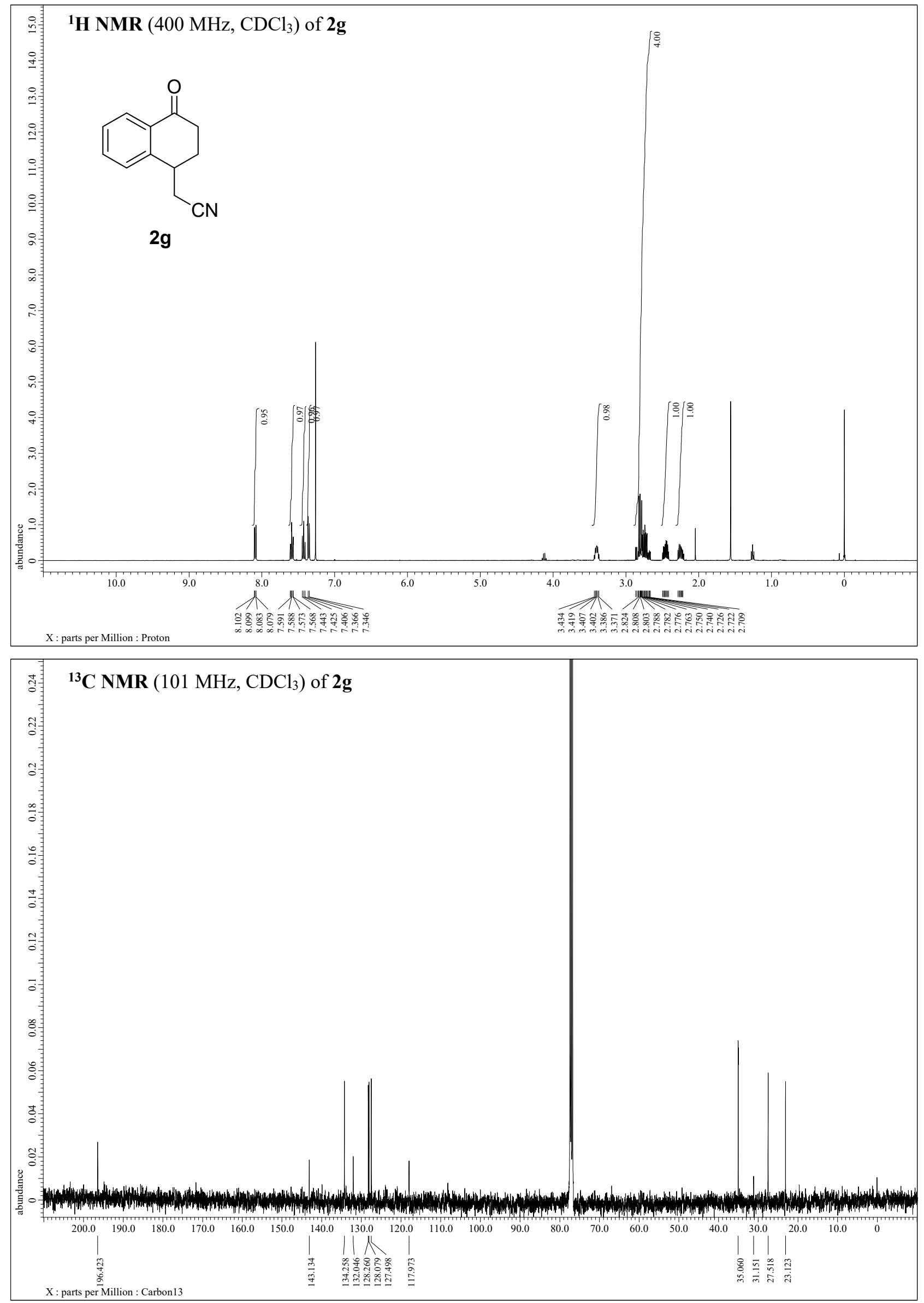

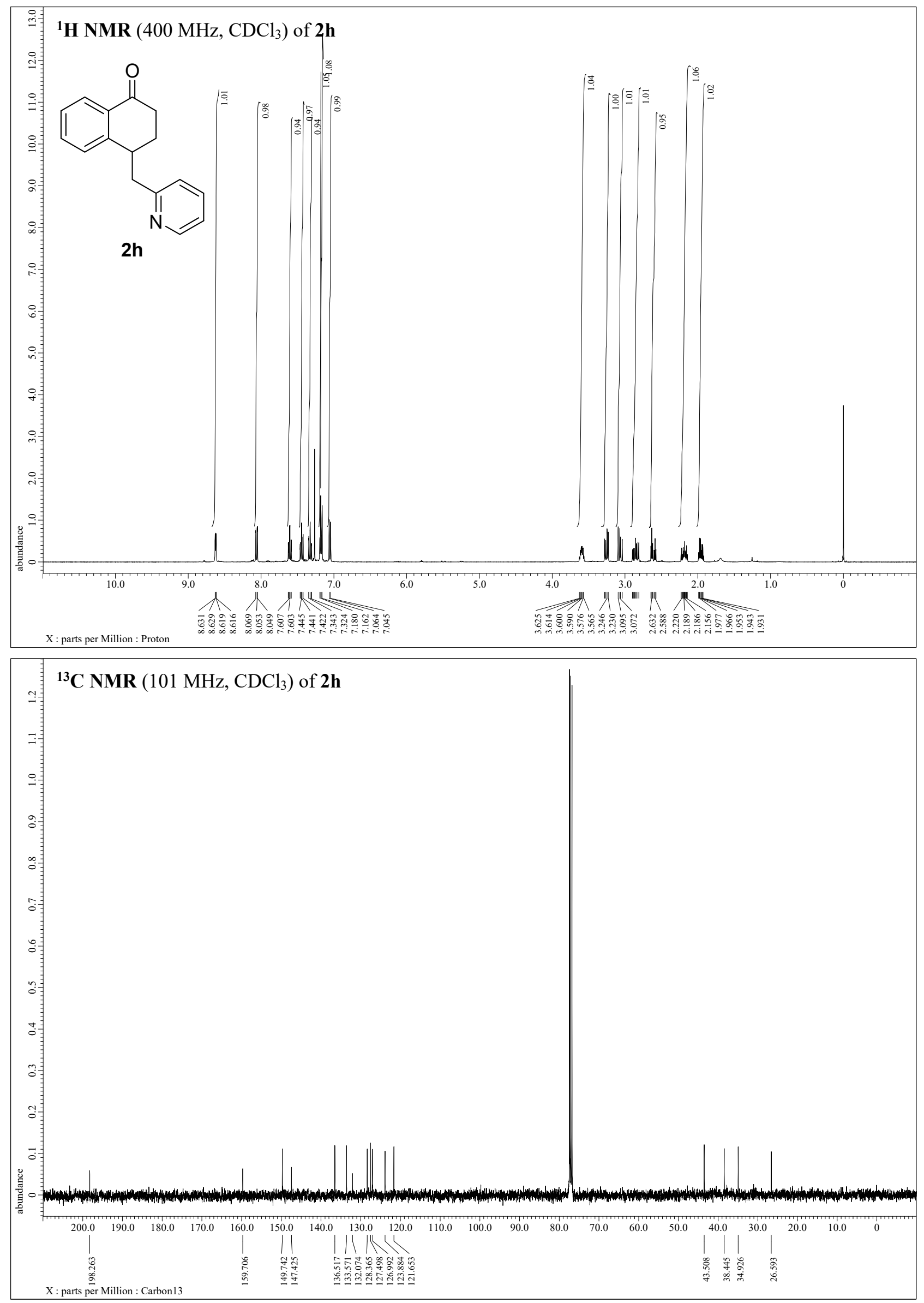

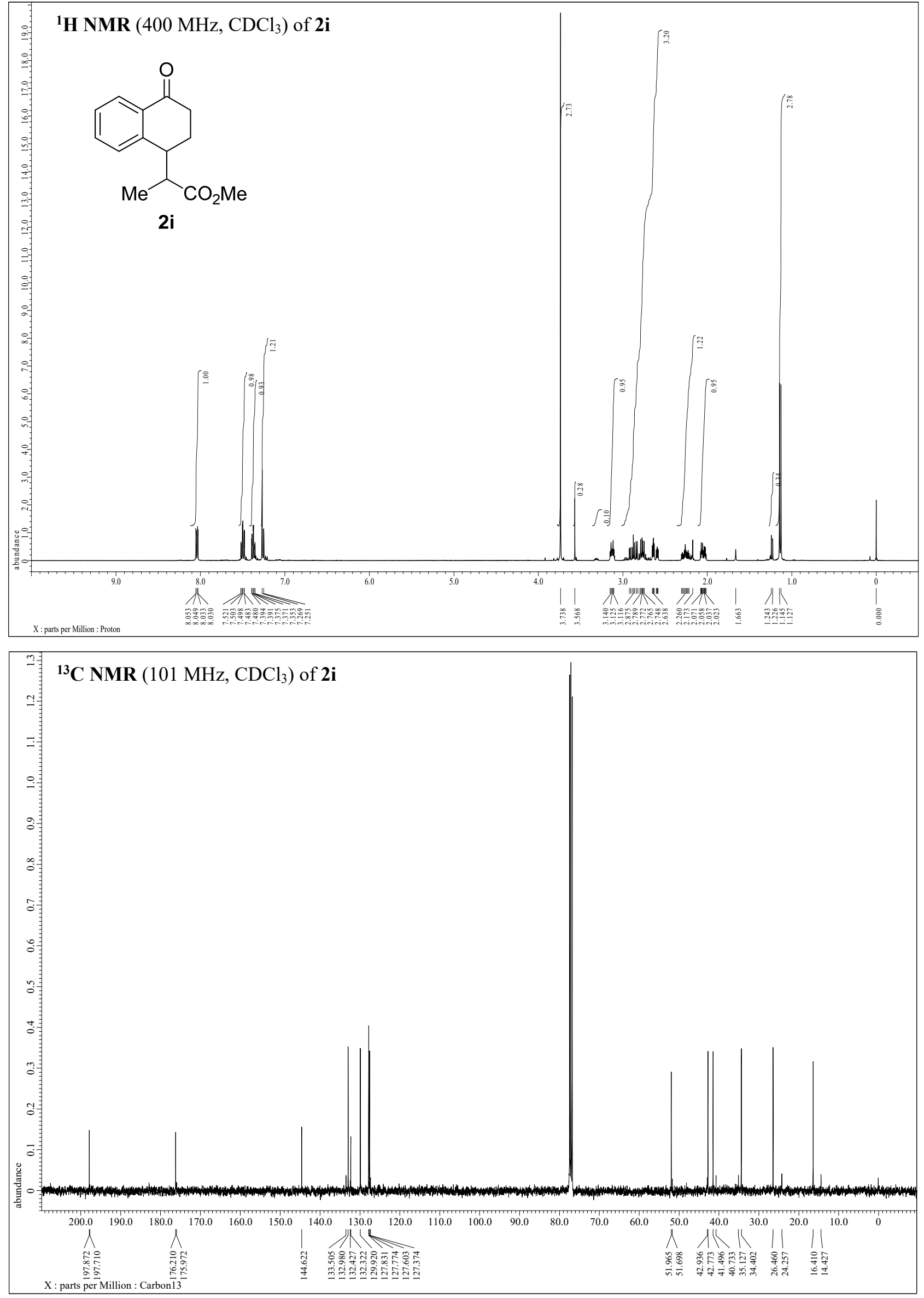

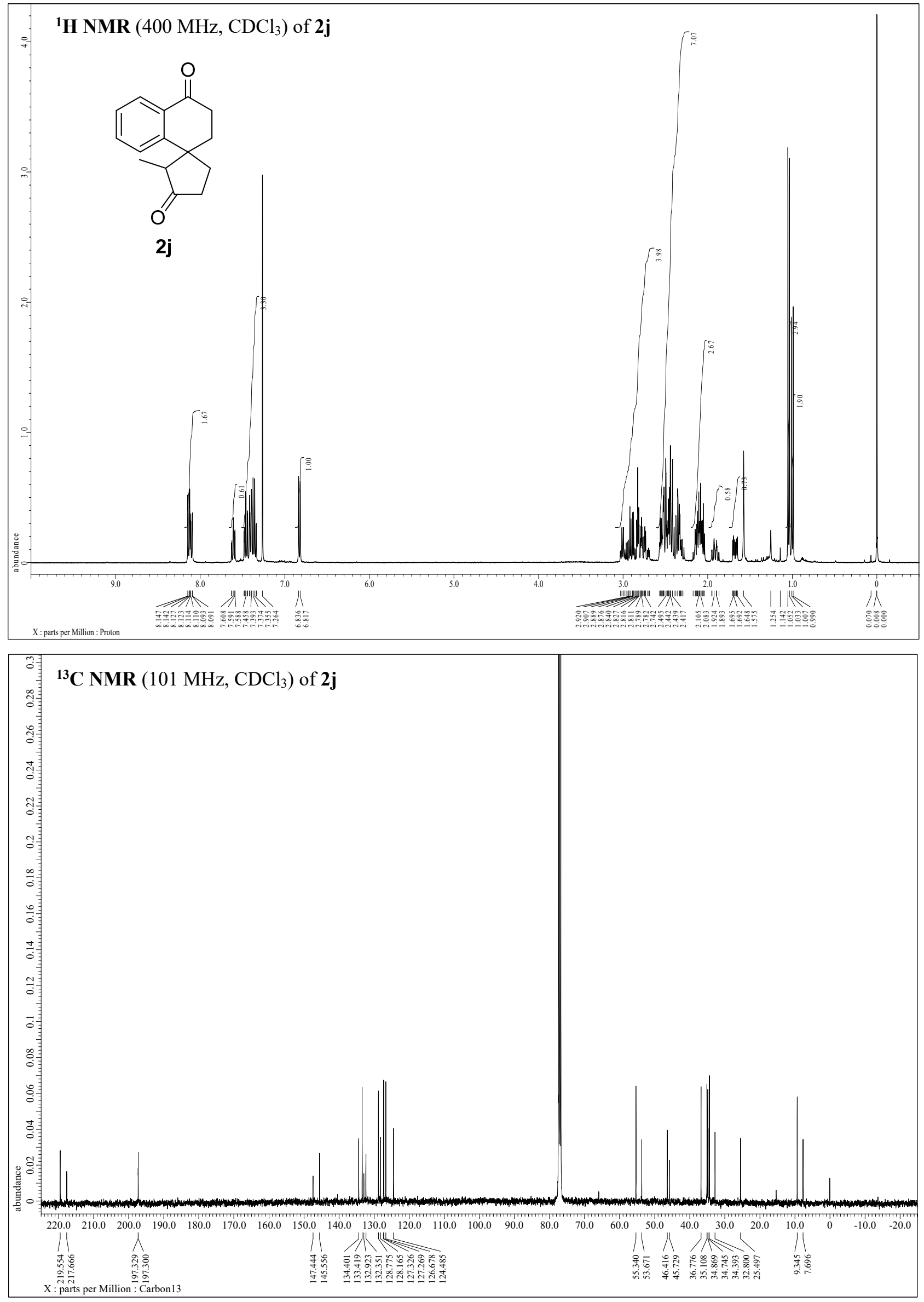

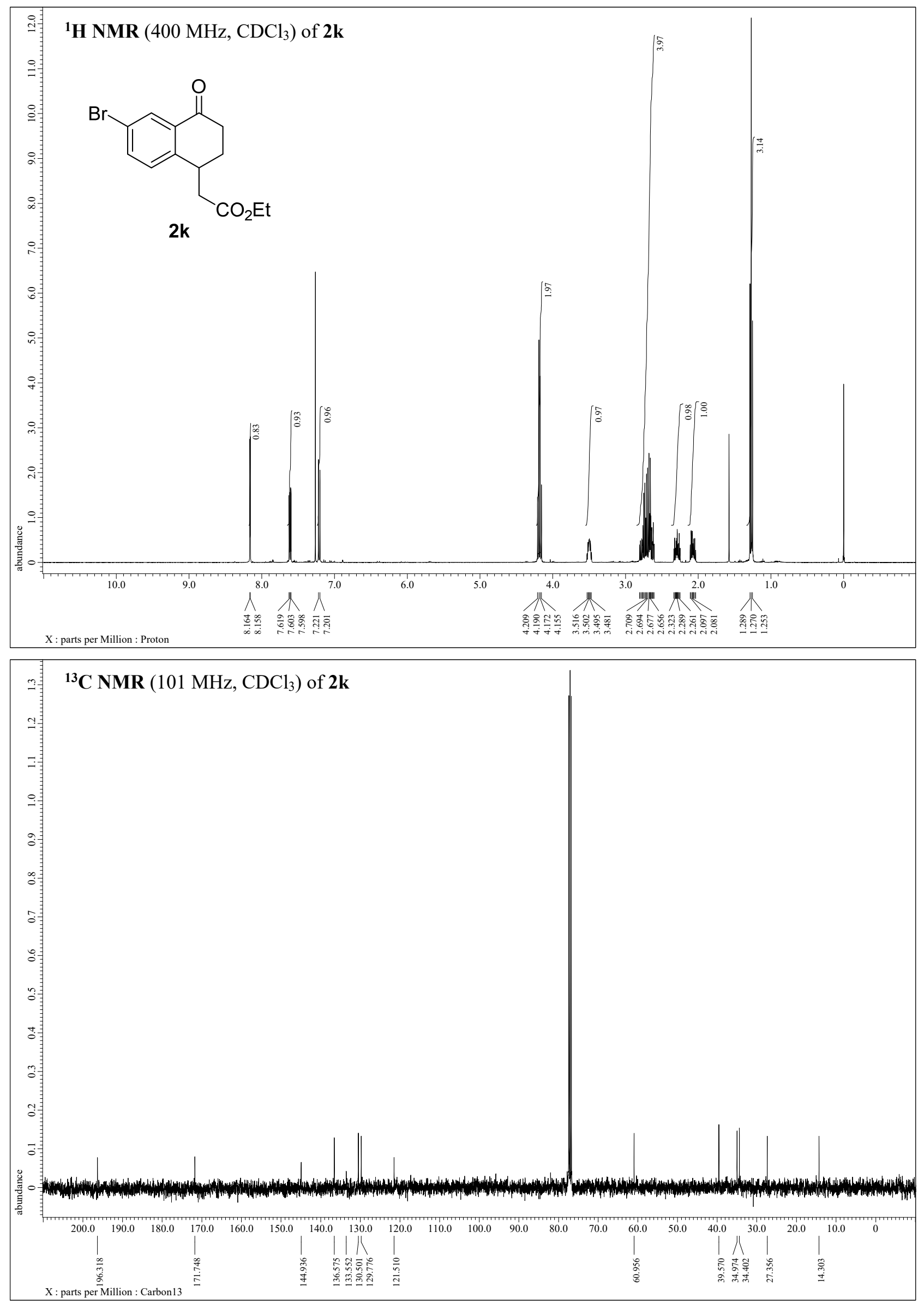

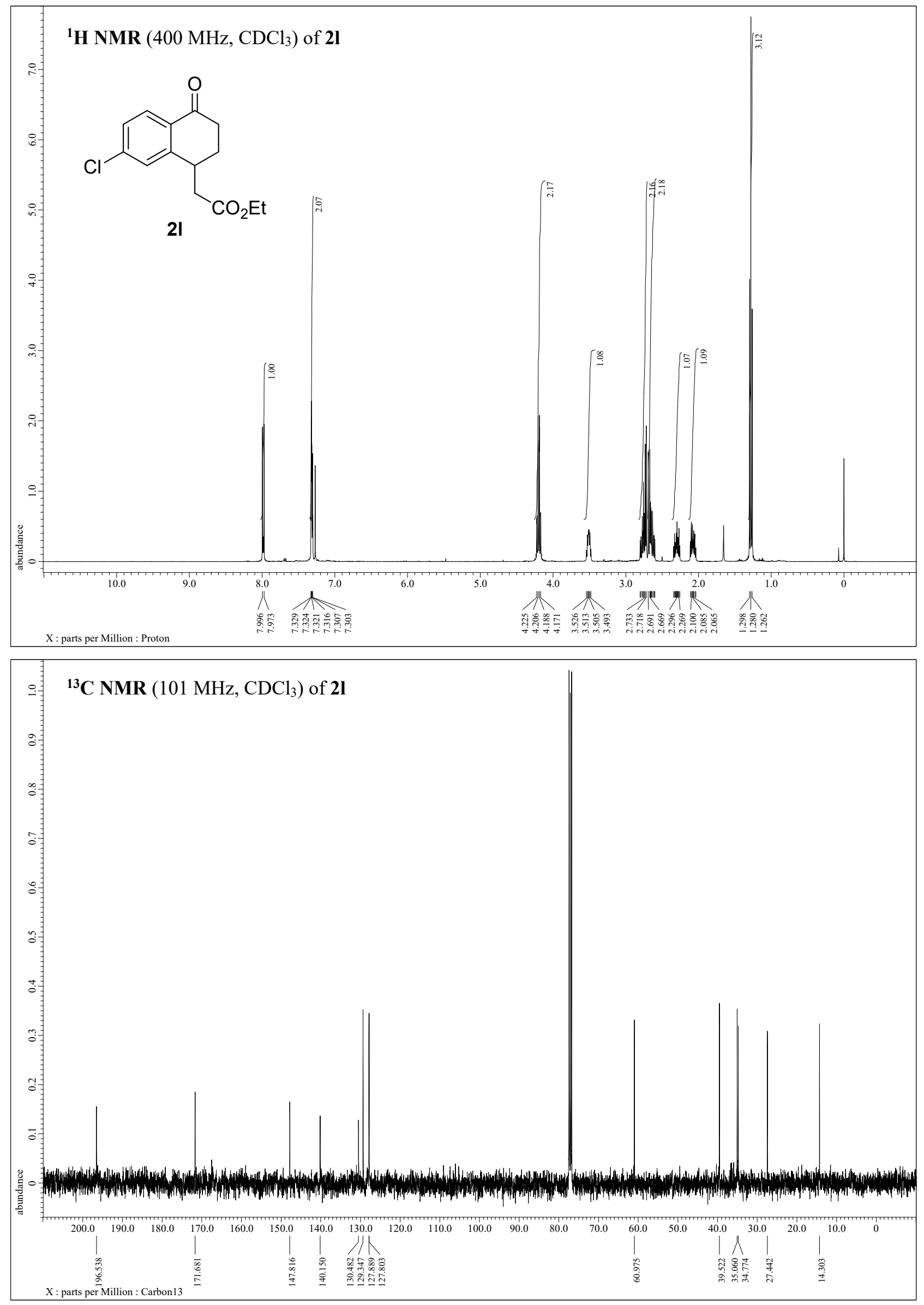

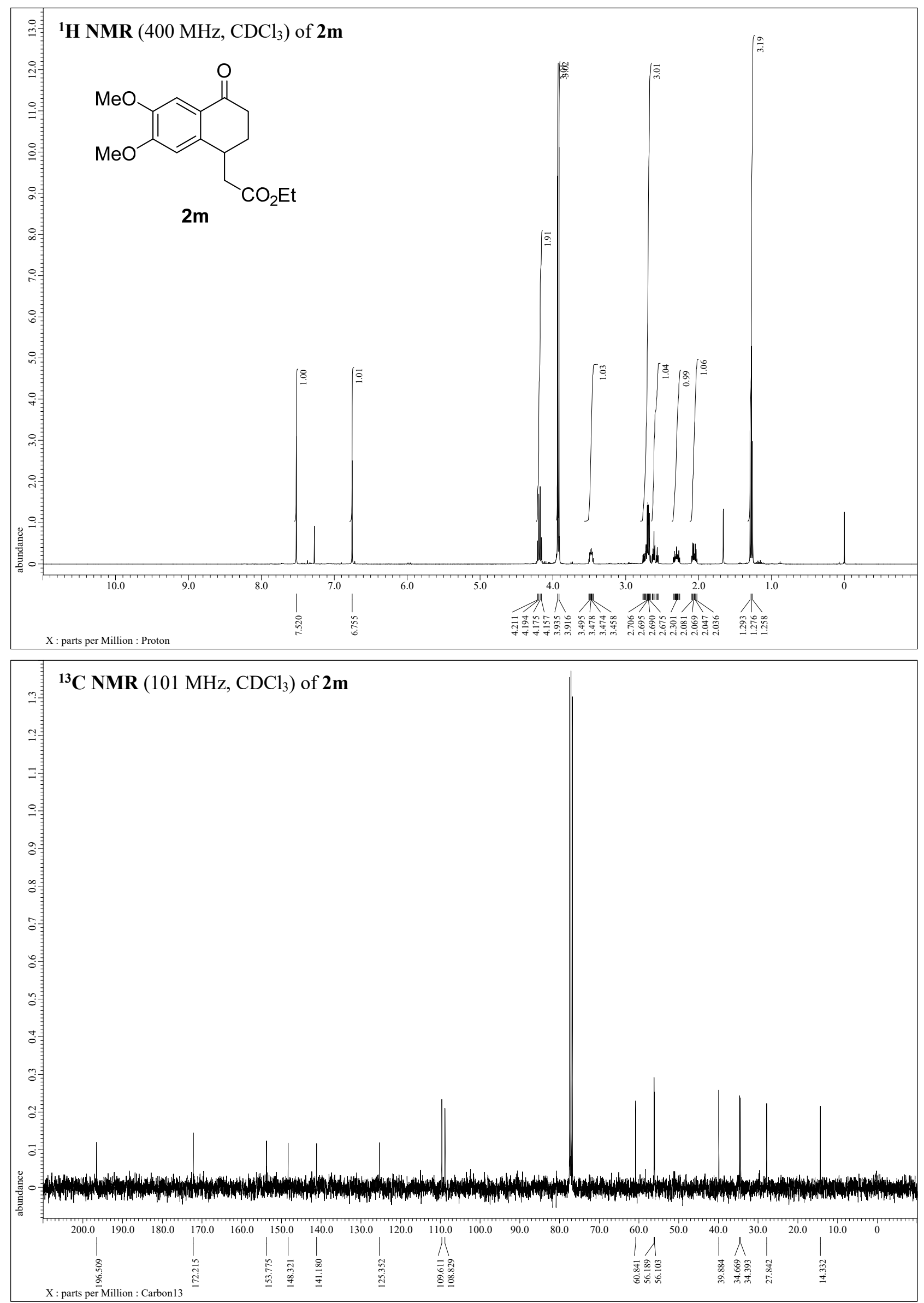


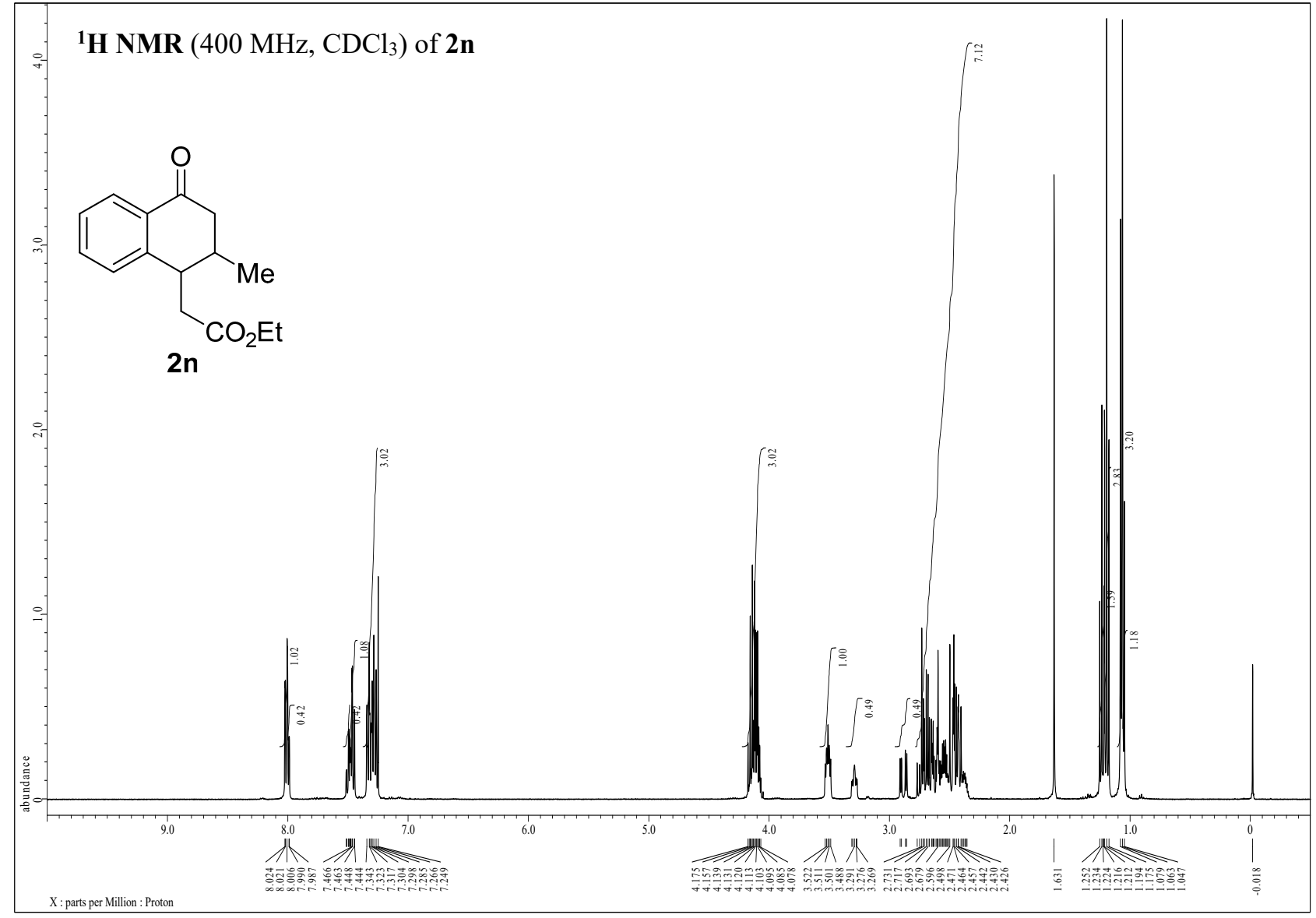

${ }^{13} \mathbf{C}$ NMR $\left(101 \mathrm{MHz}, \mathrm{CDCl}_{3}\right)$ of $\mathbf{2 n}$

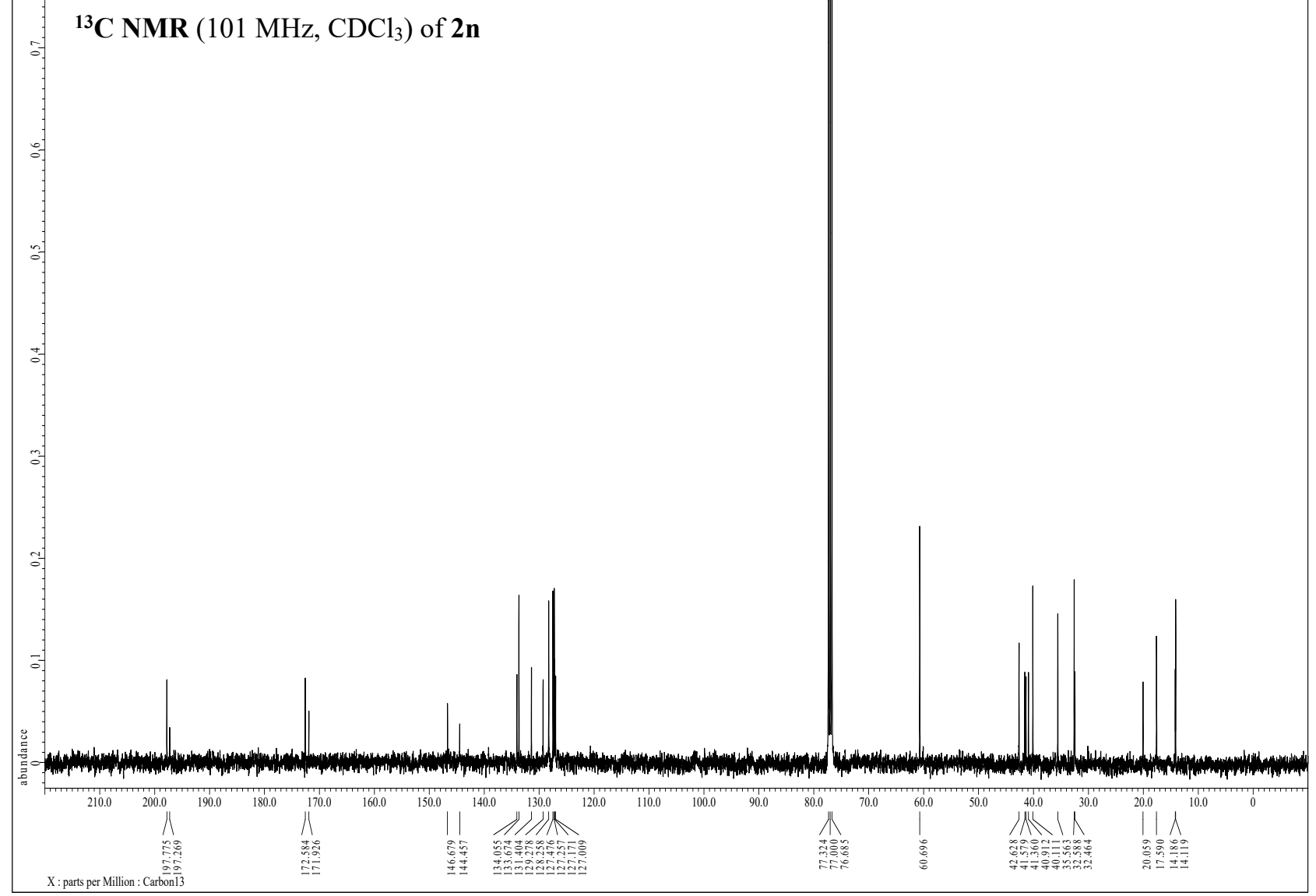



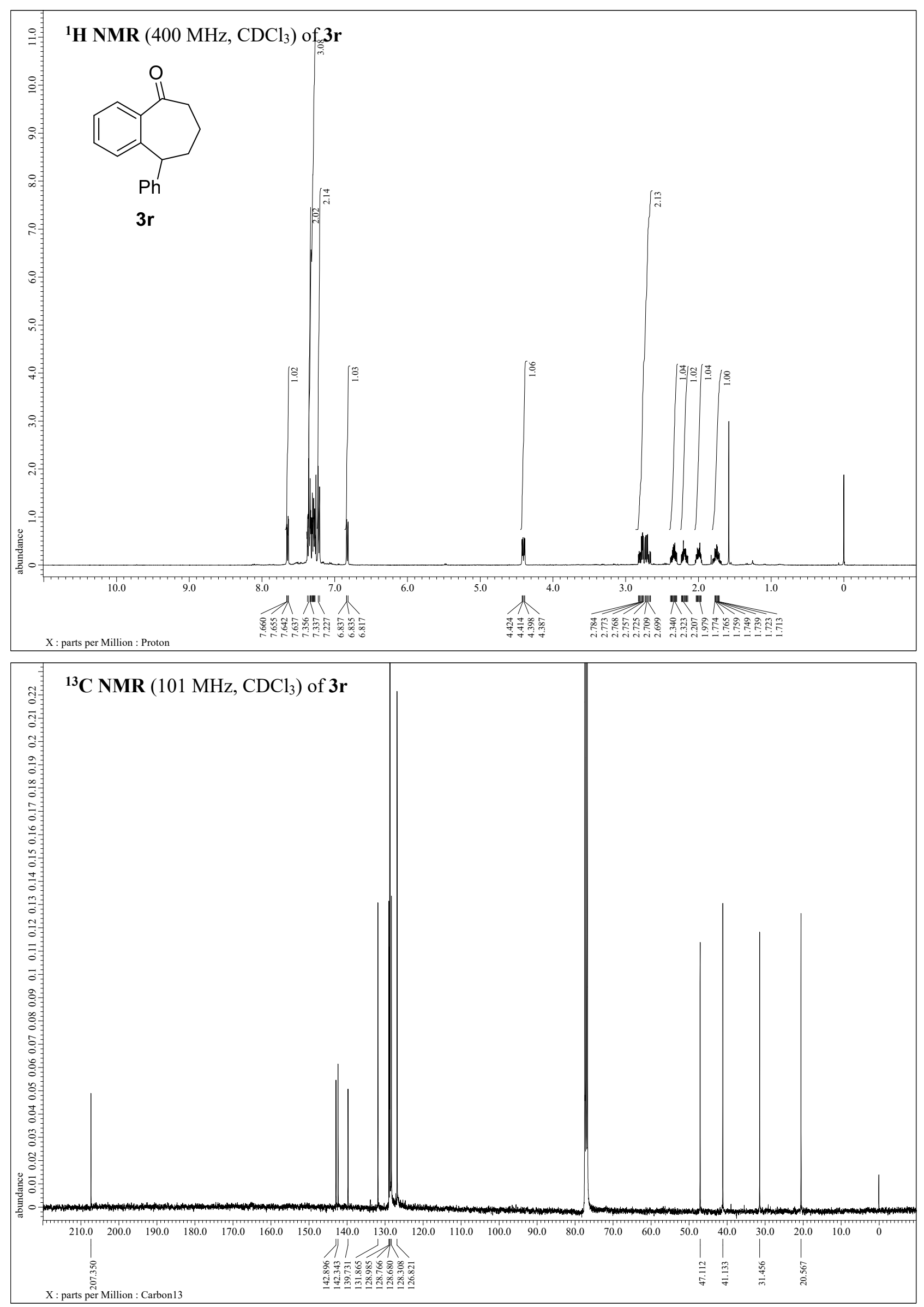

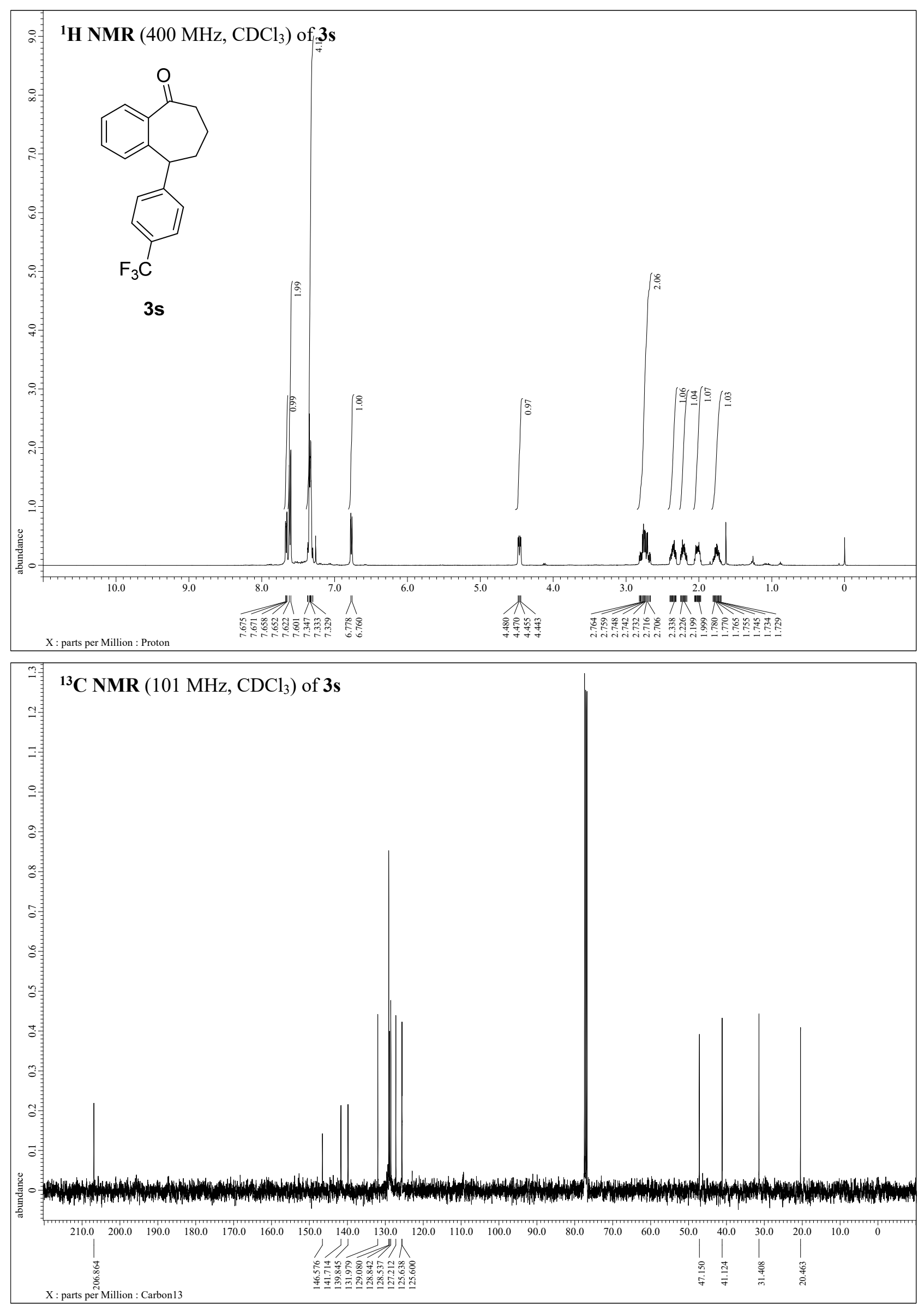

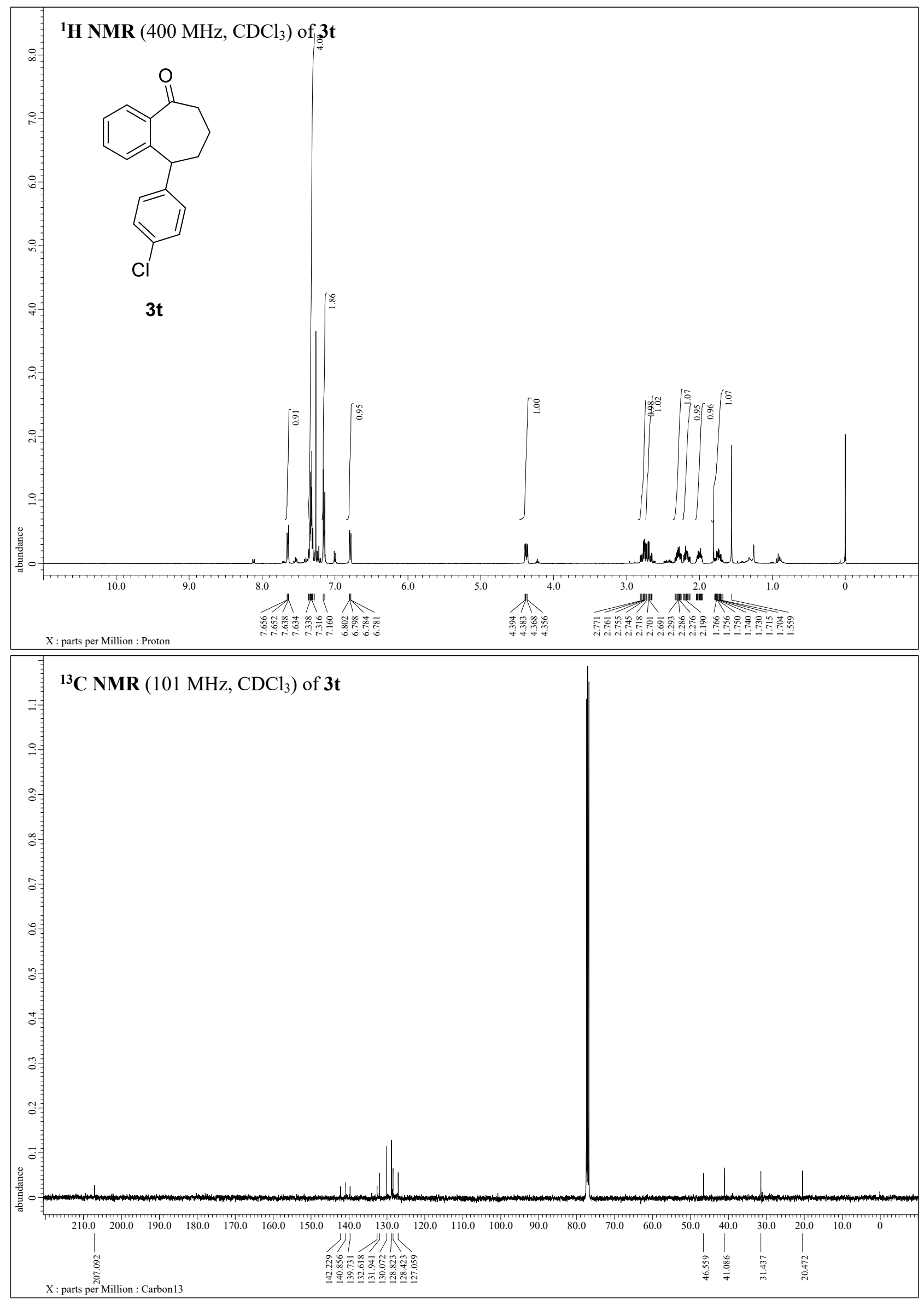

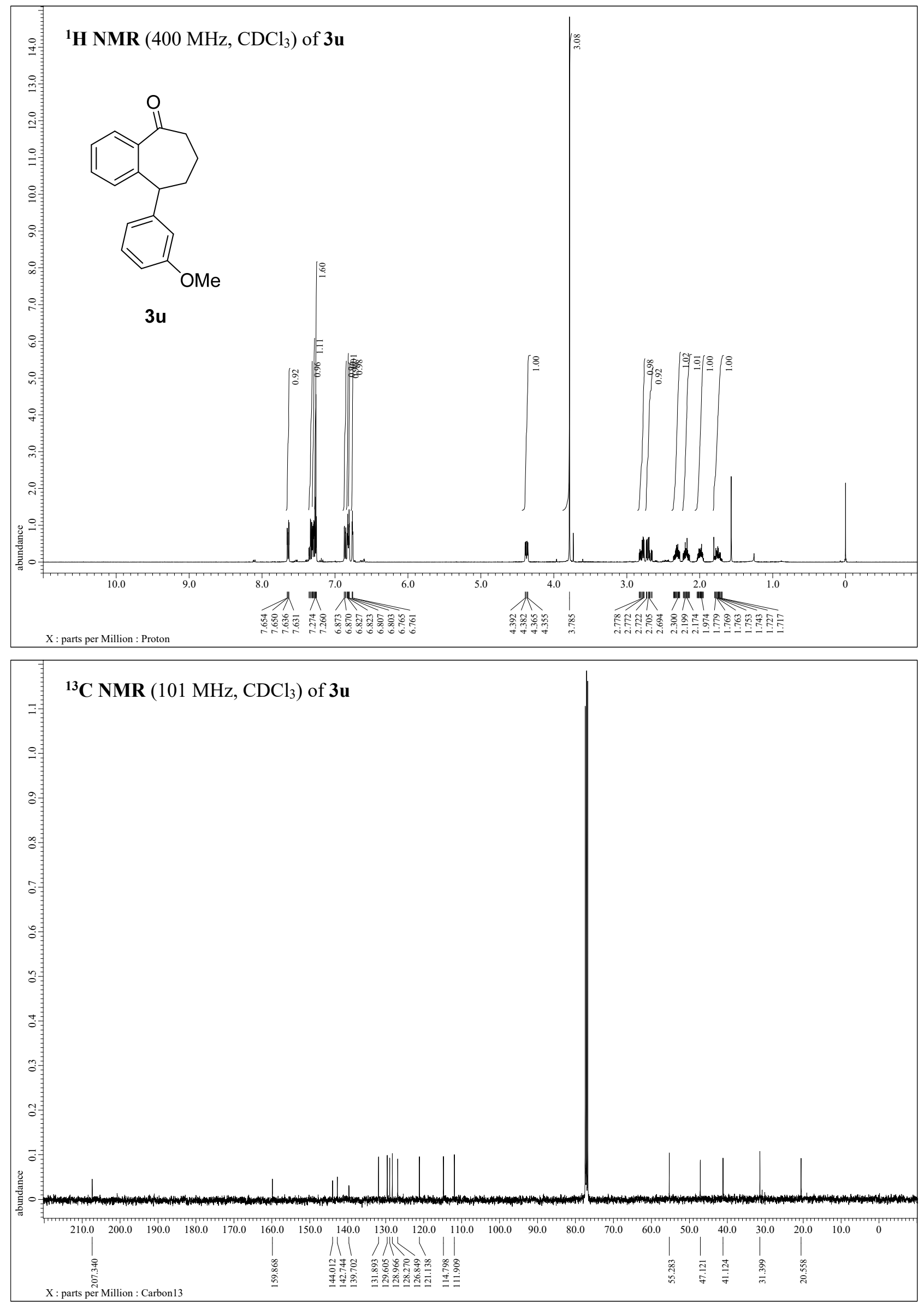

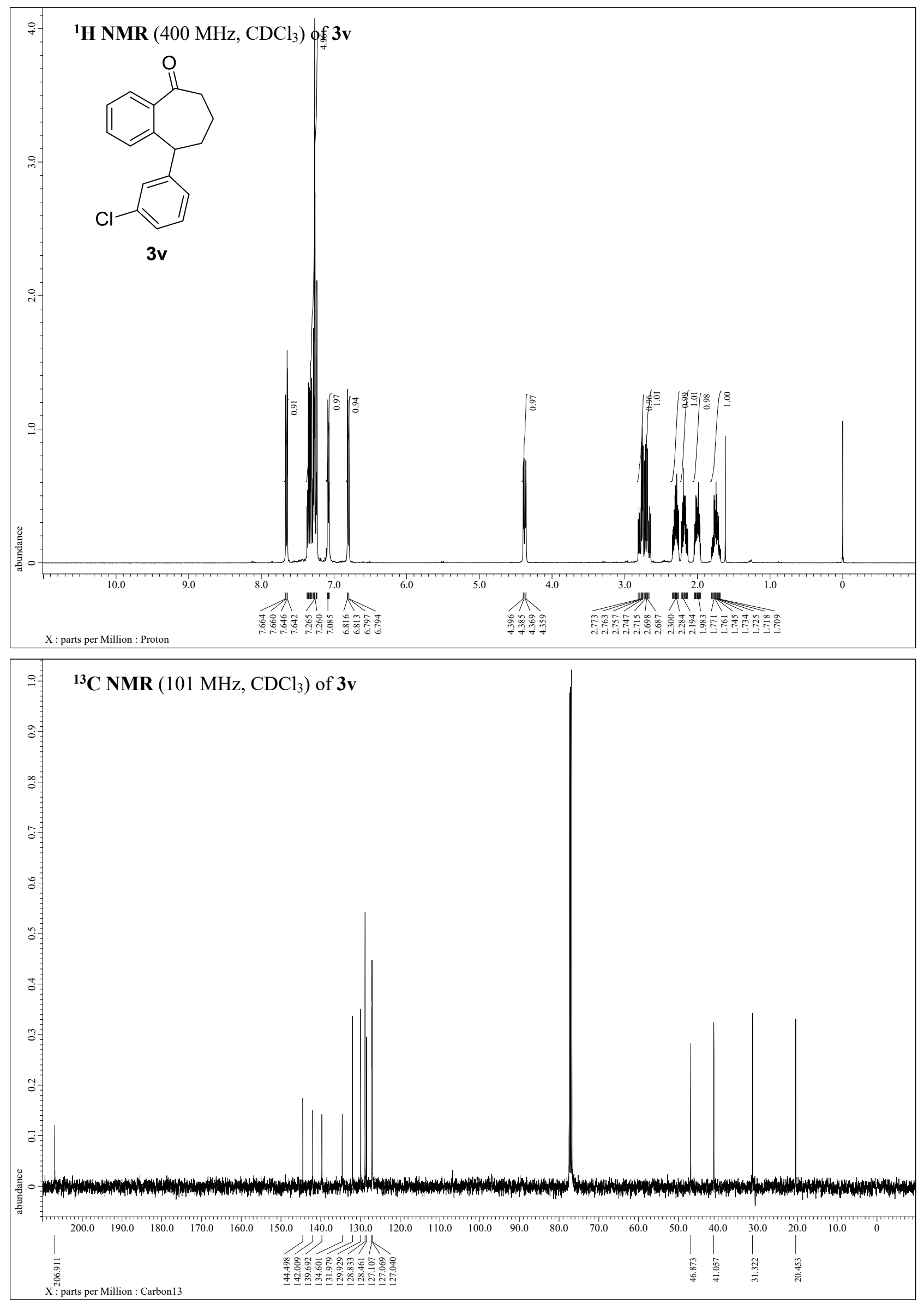

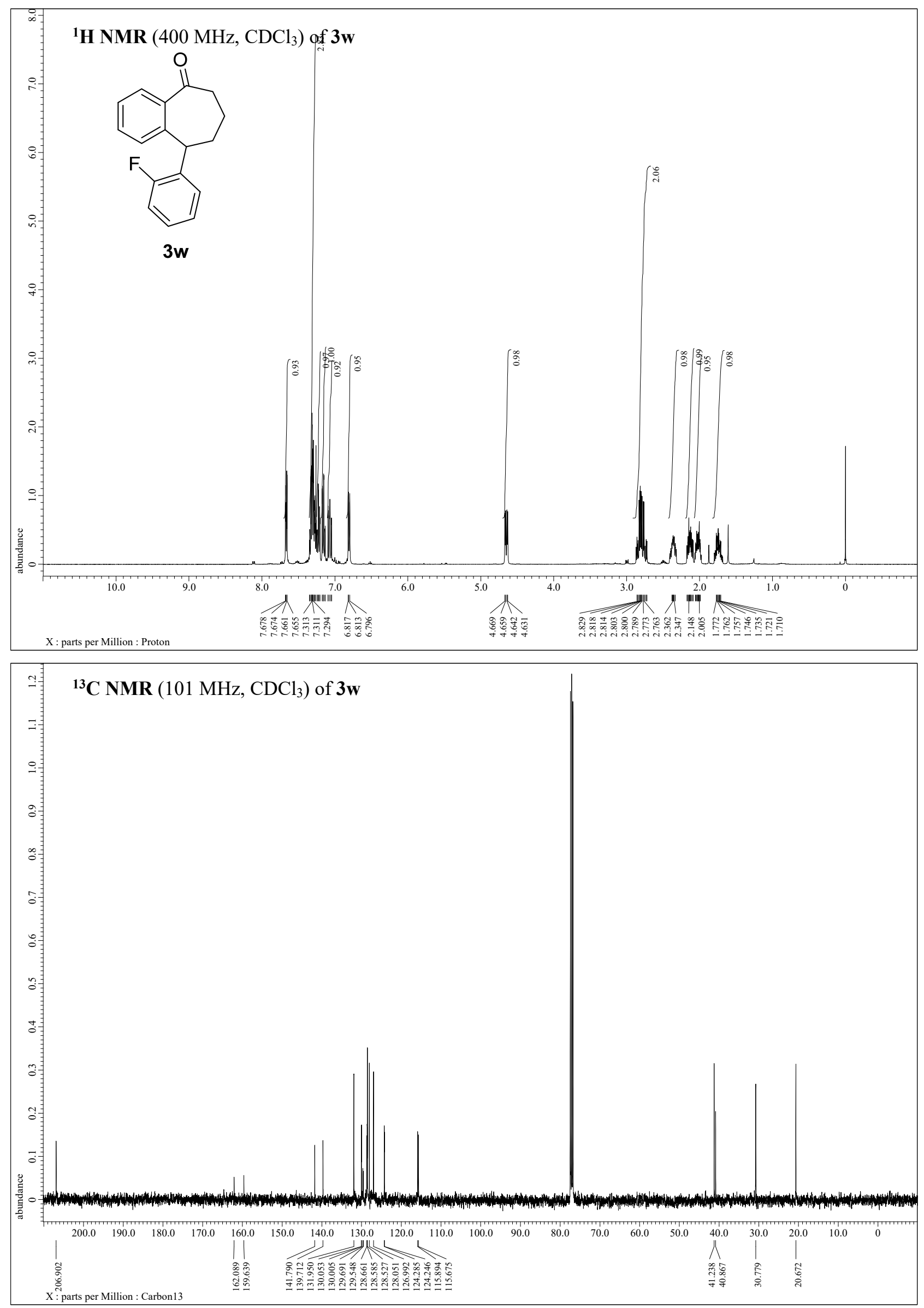

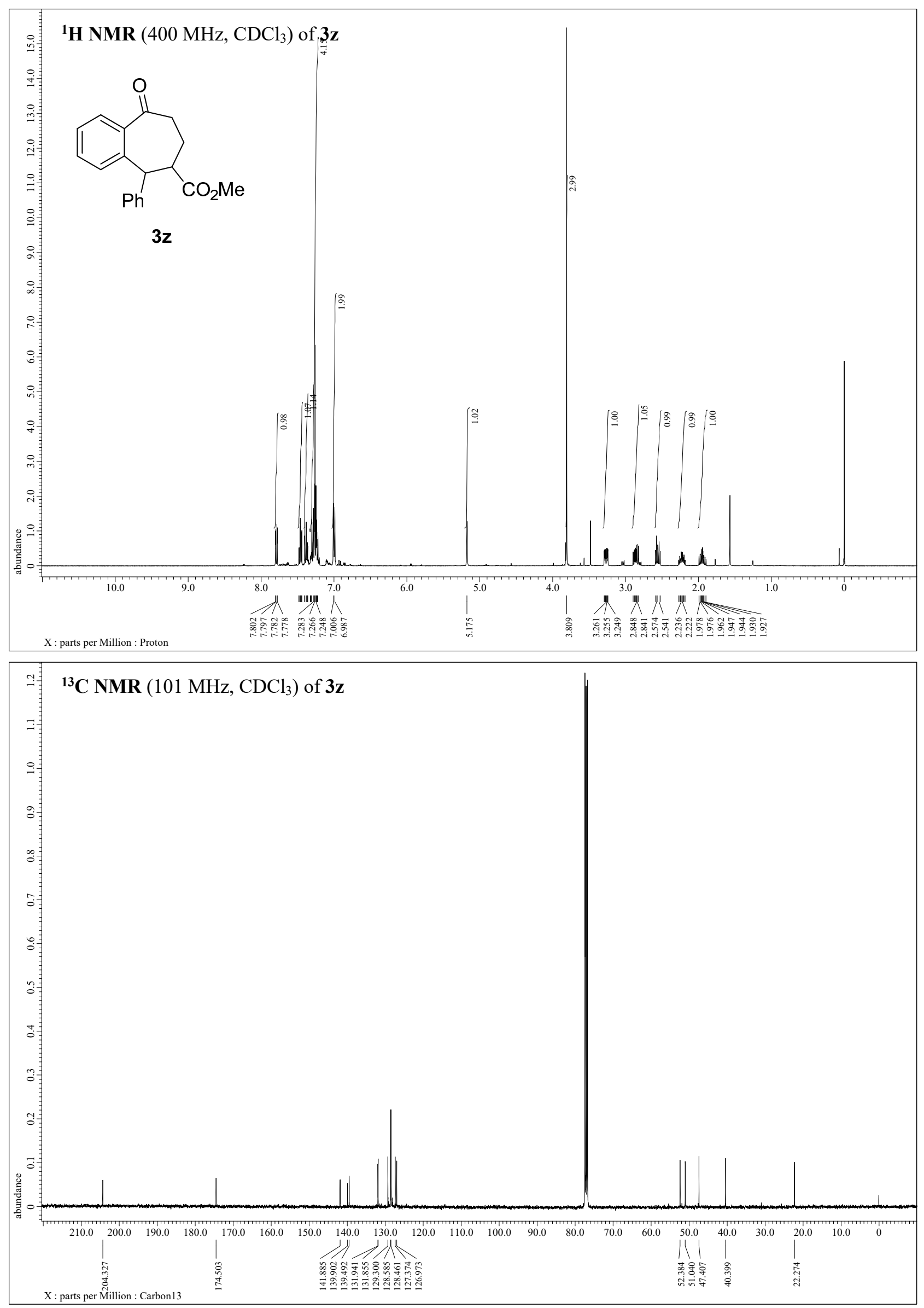

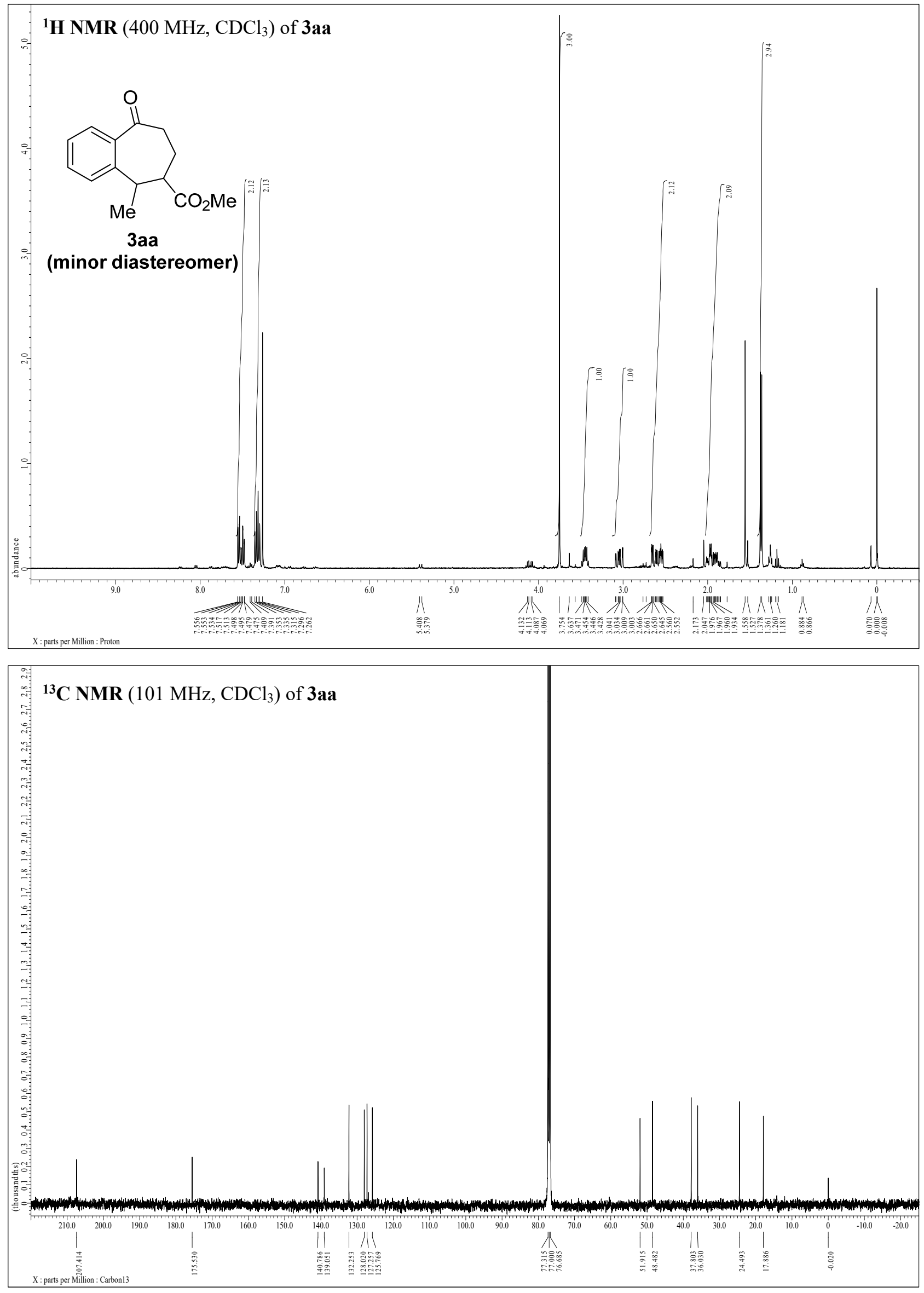

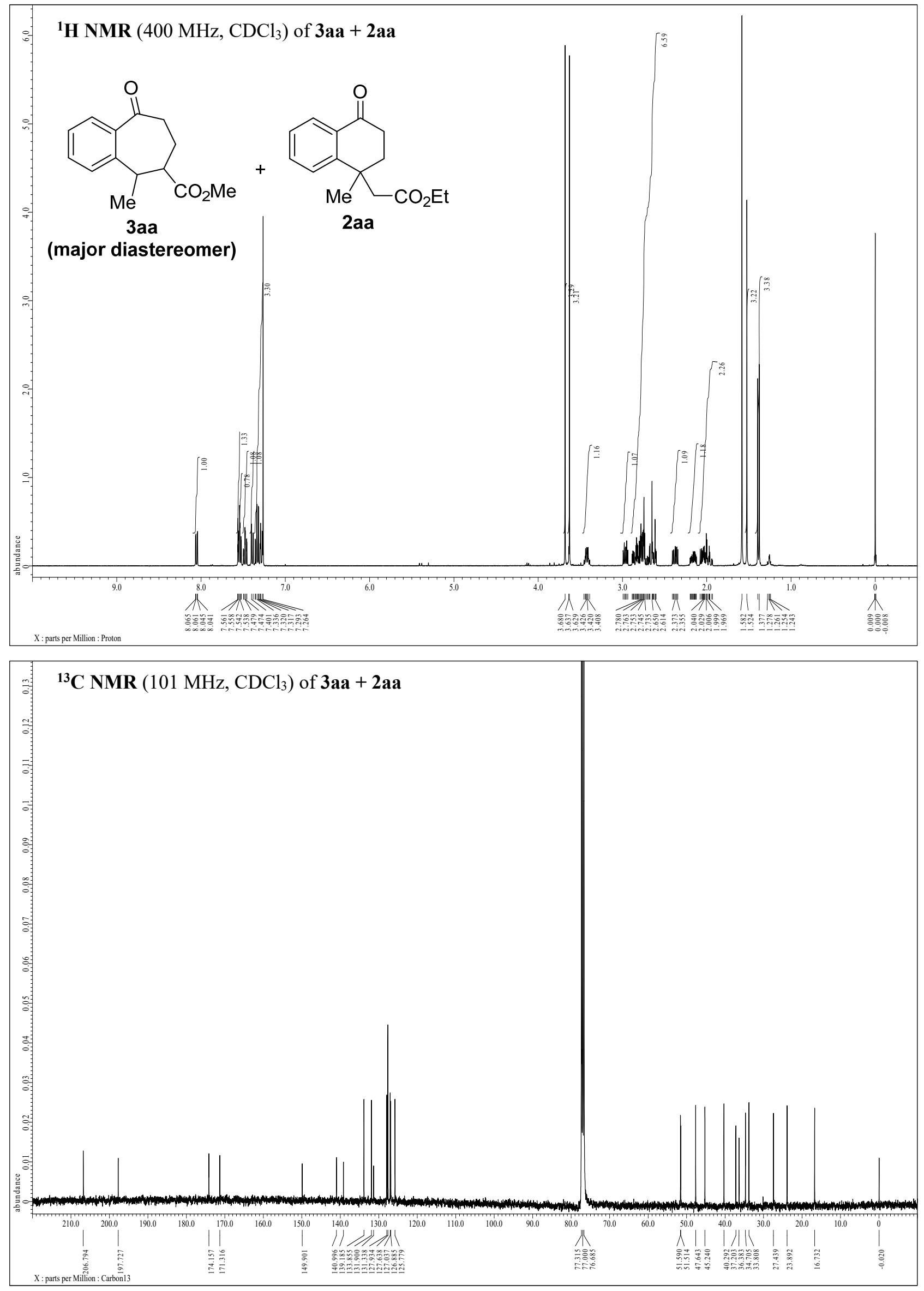


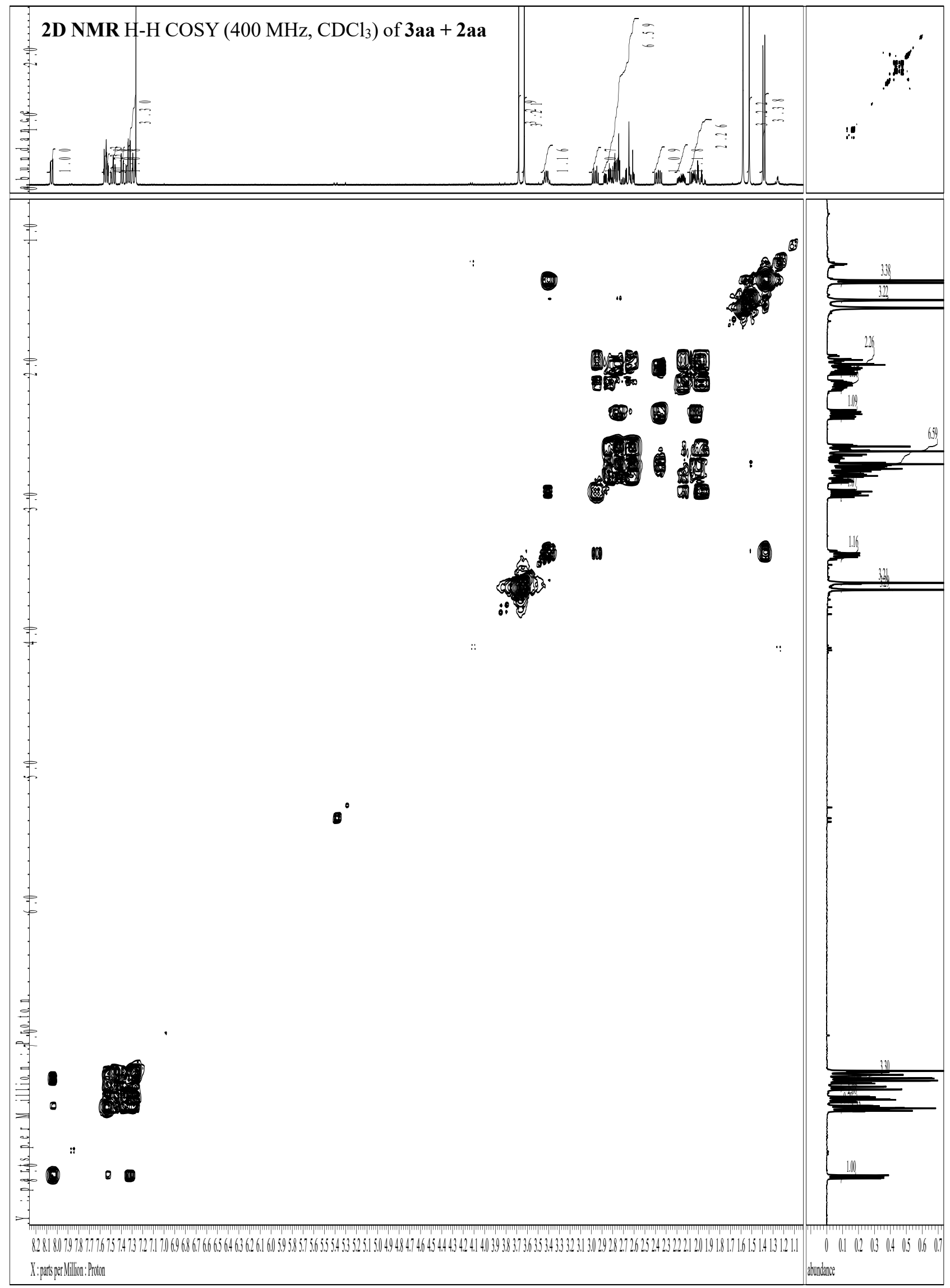



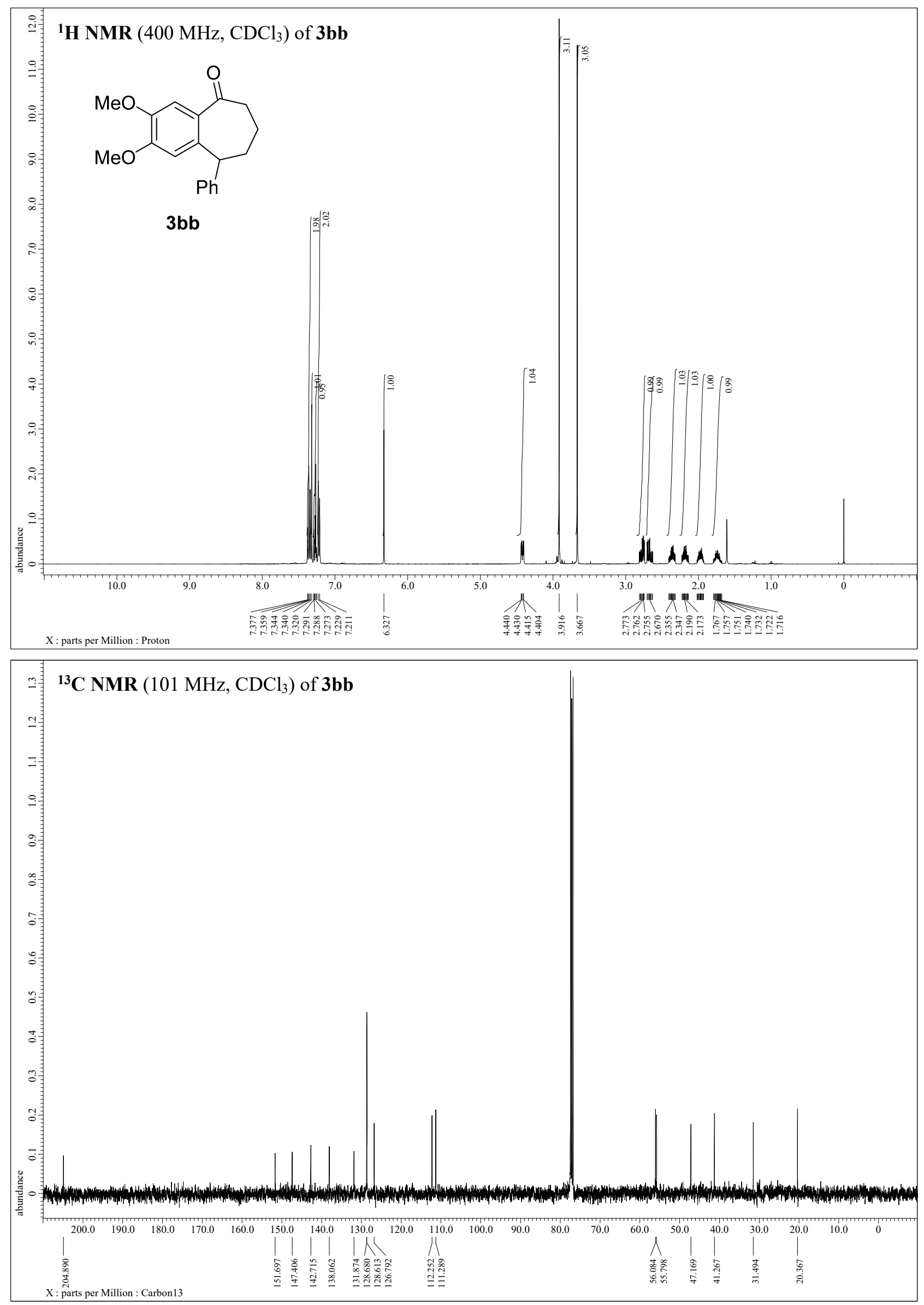

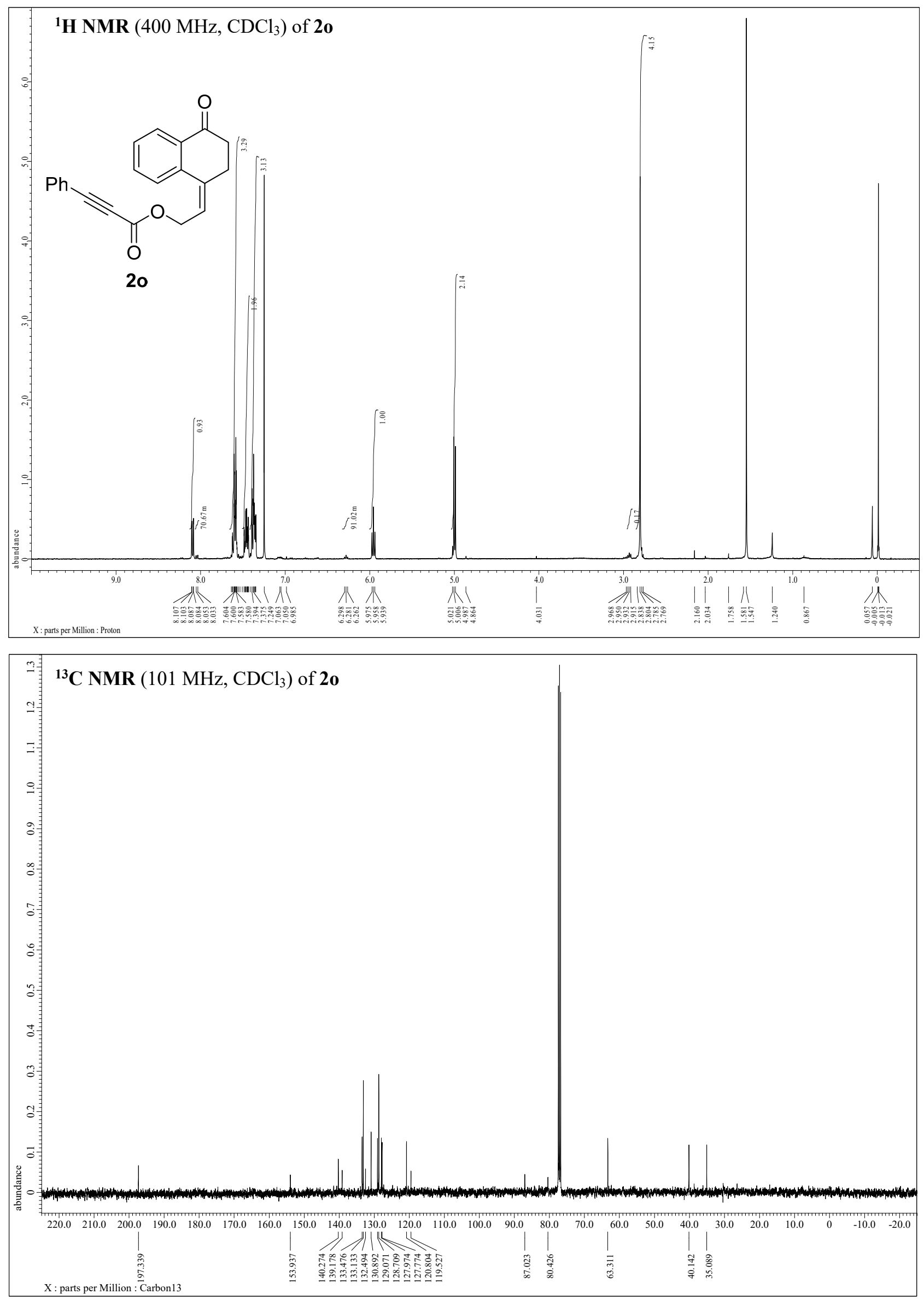

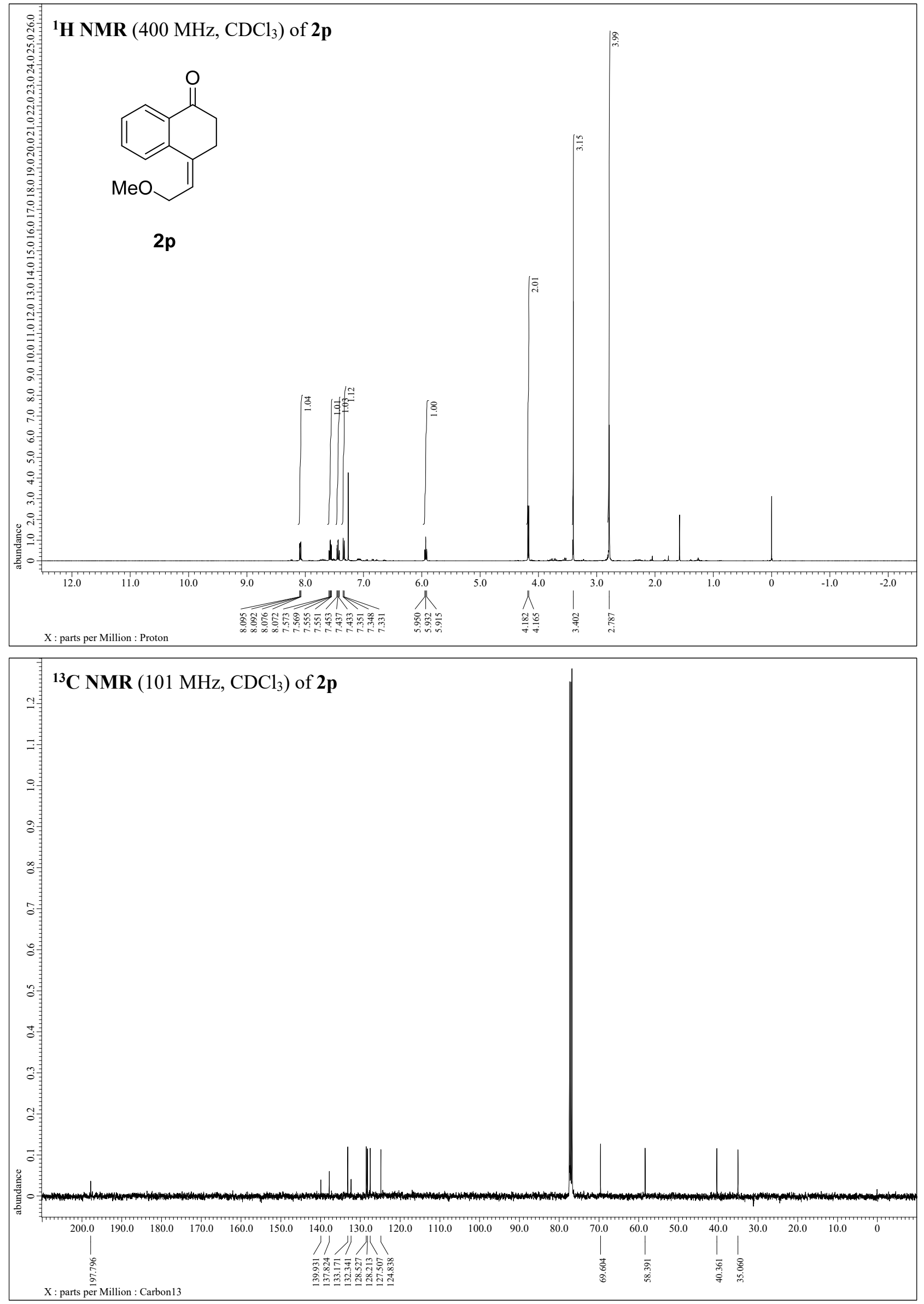

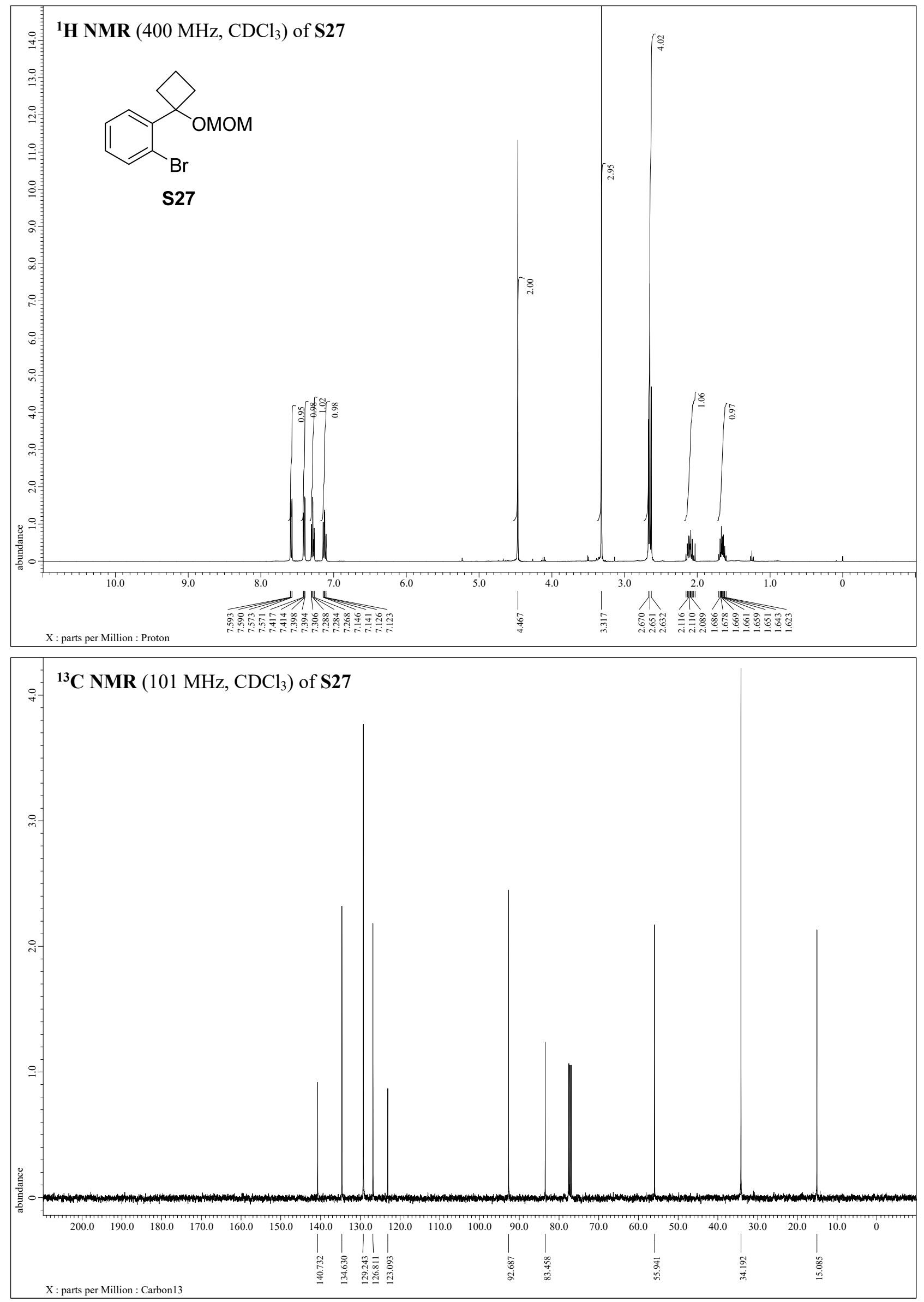

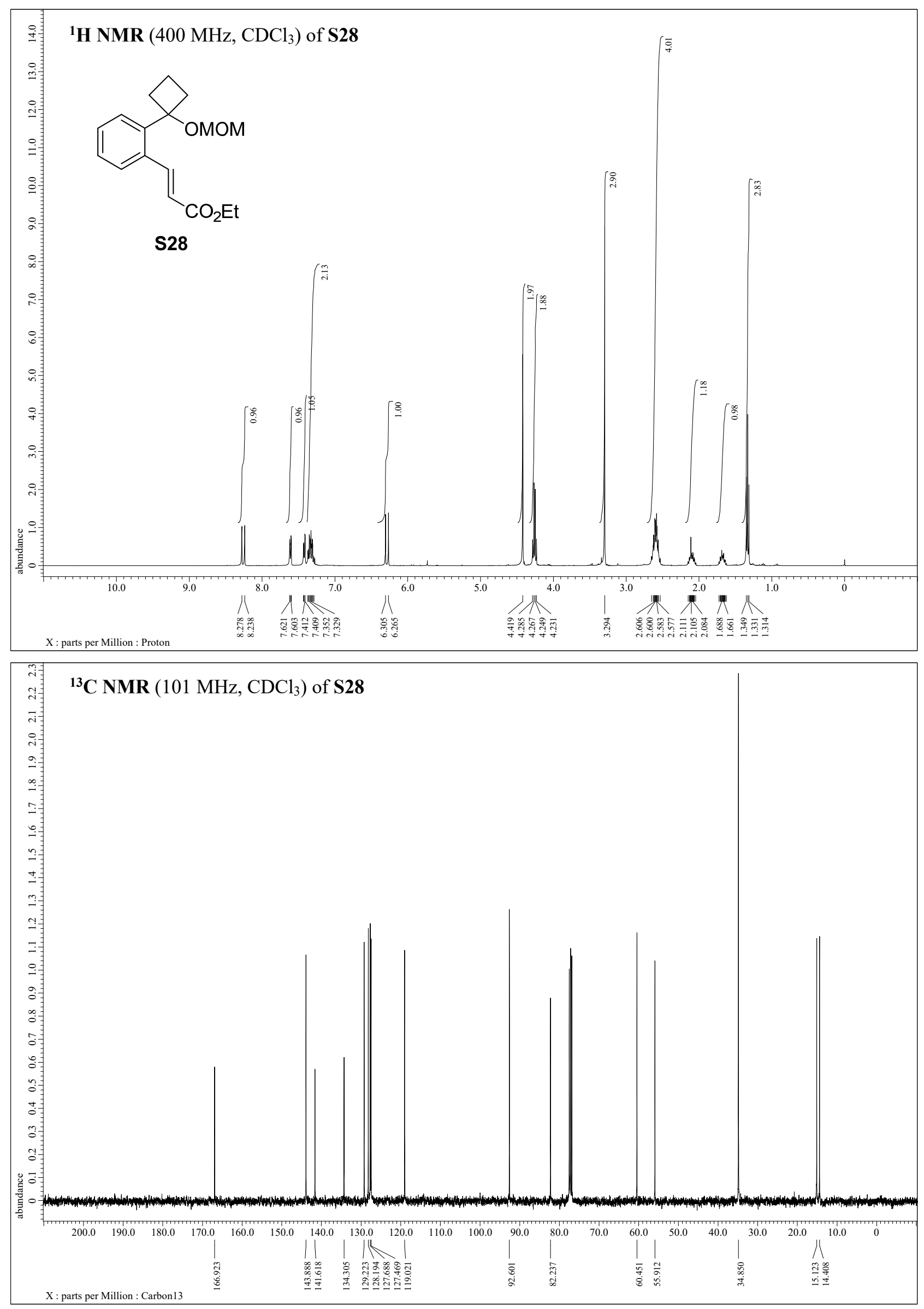

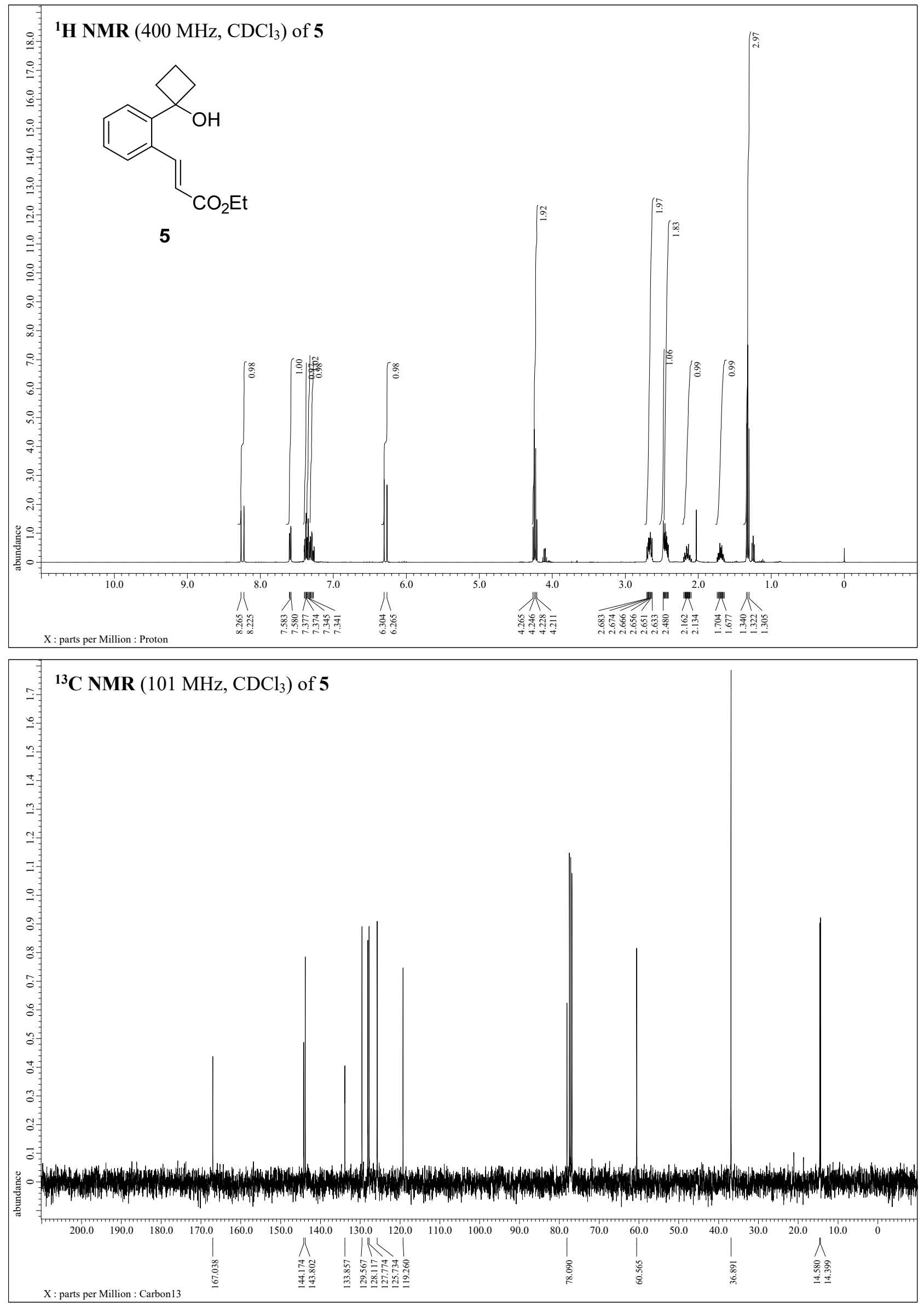

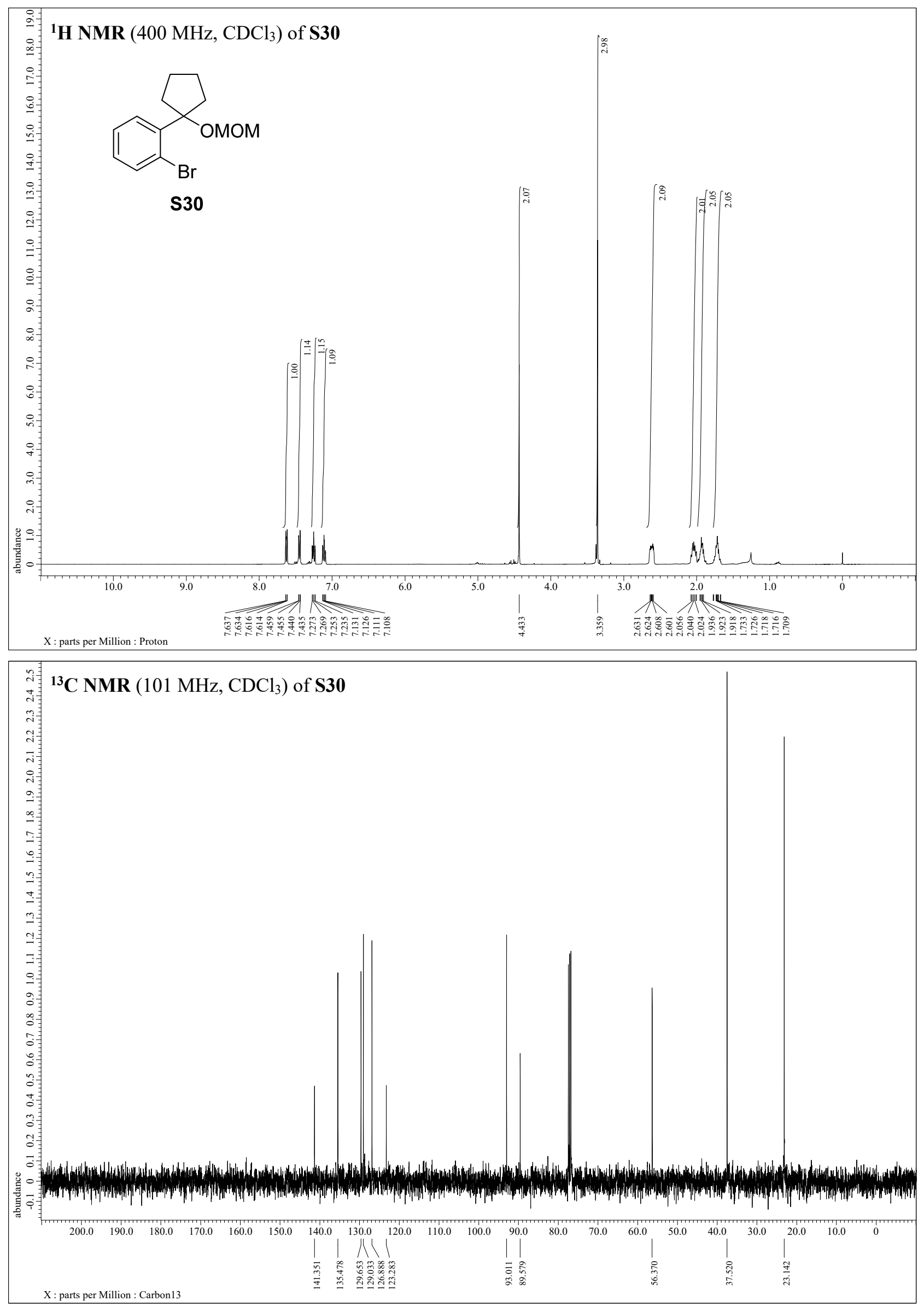

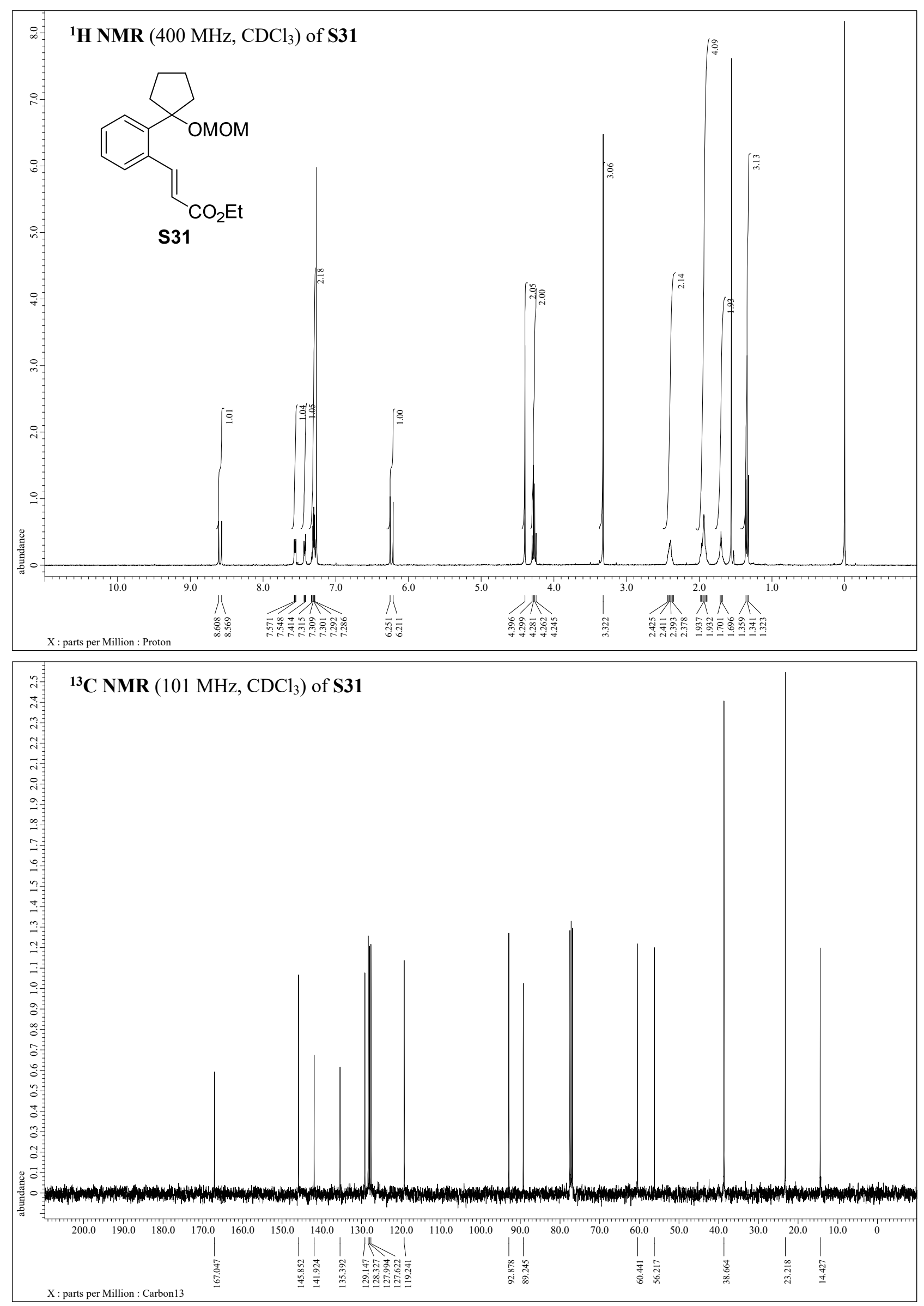

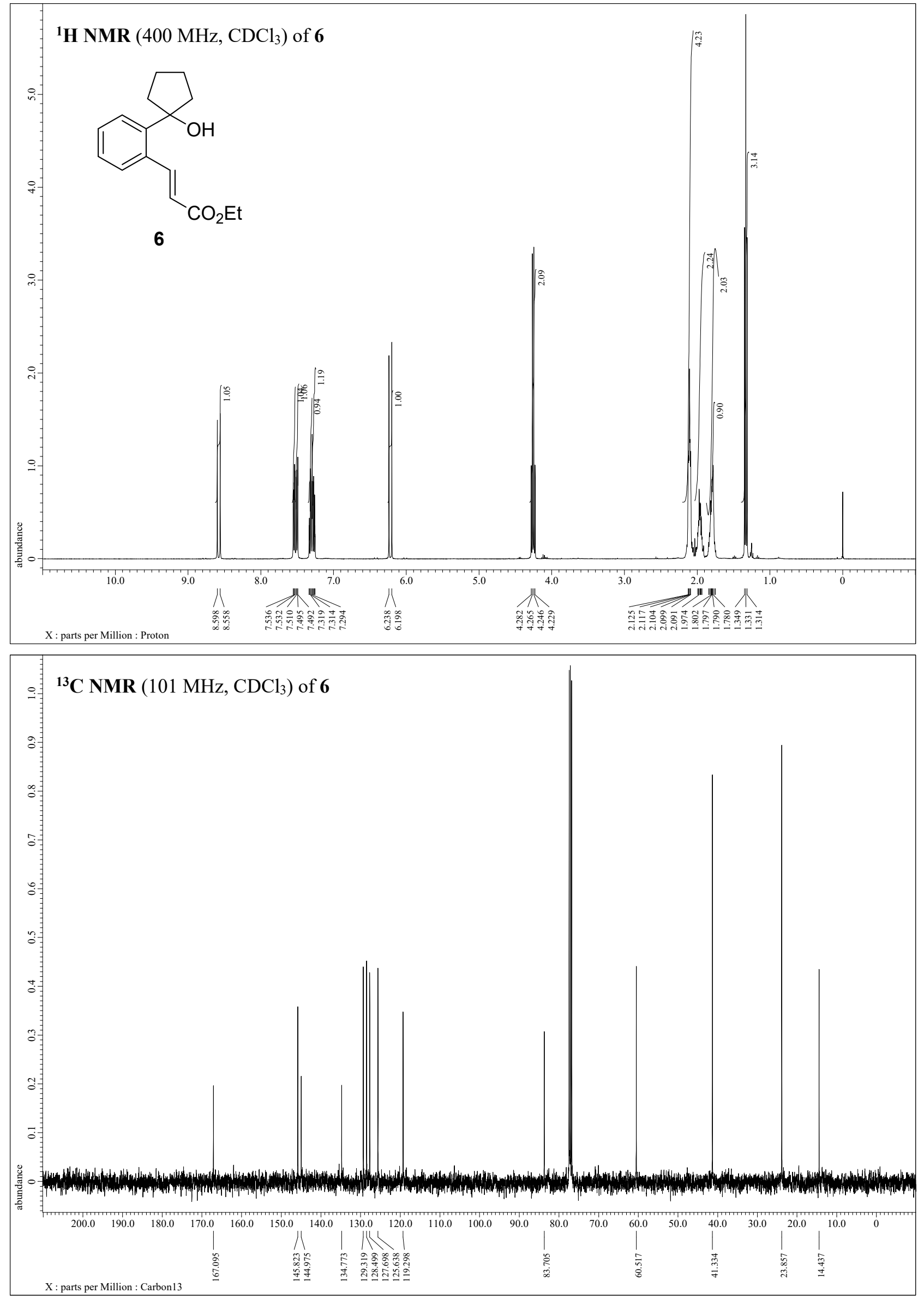

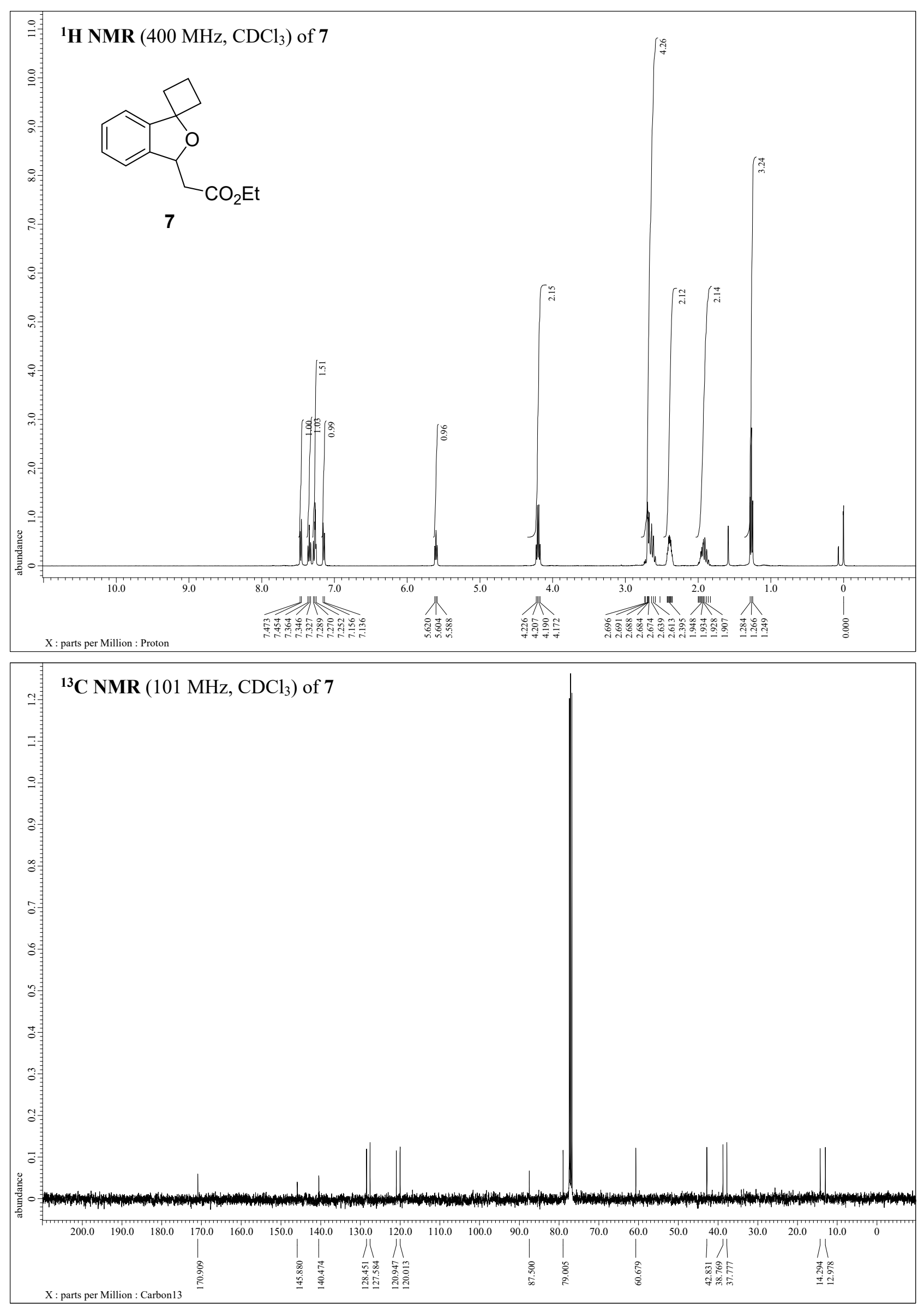

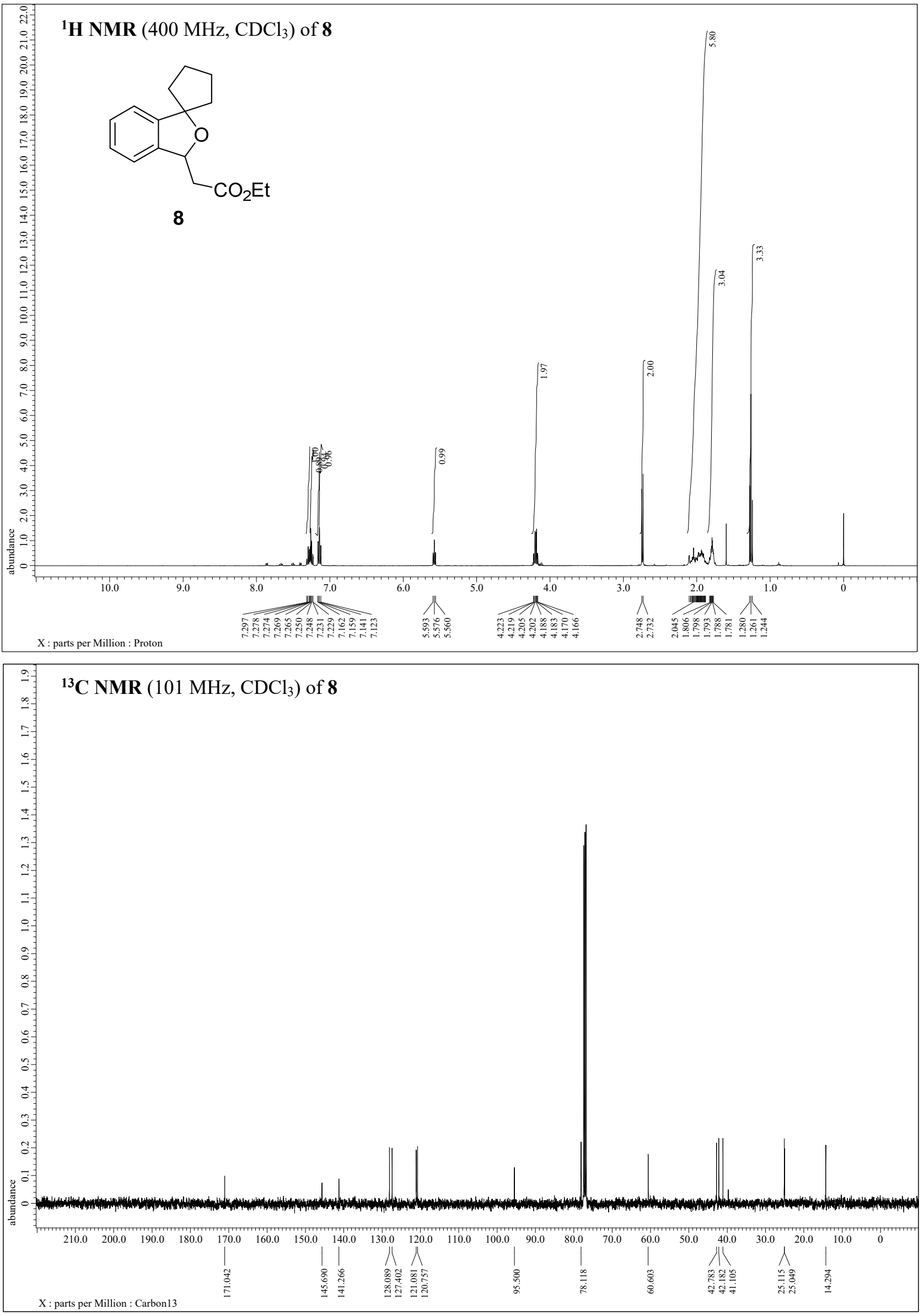

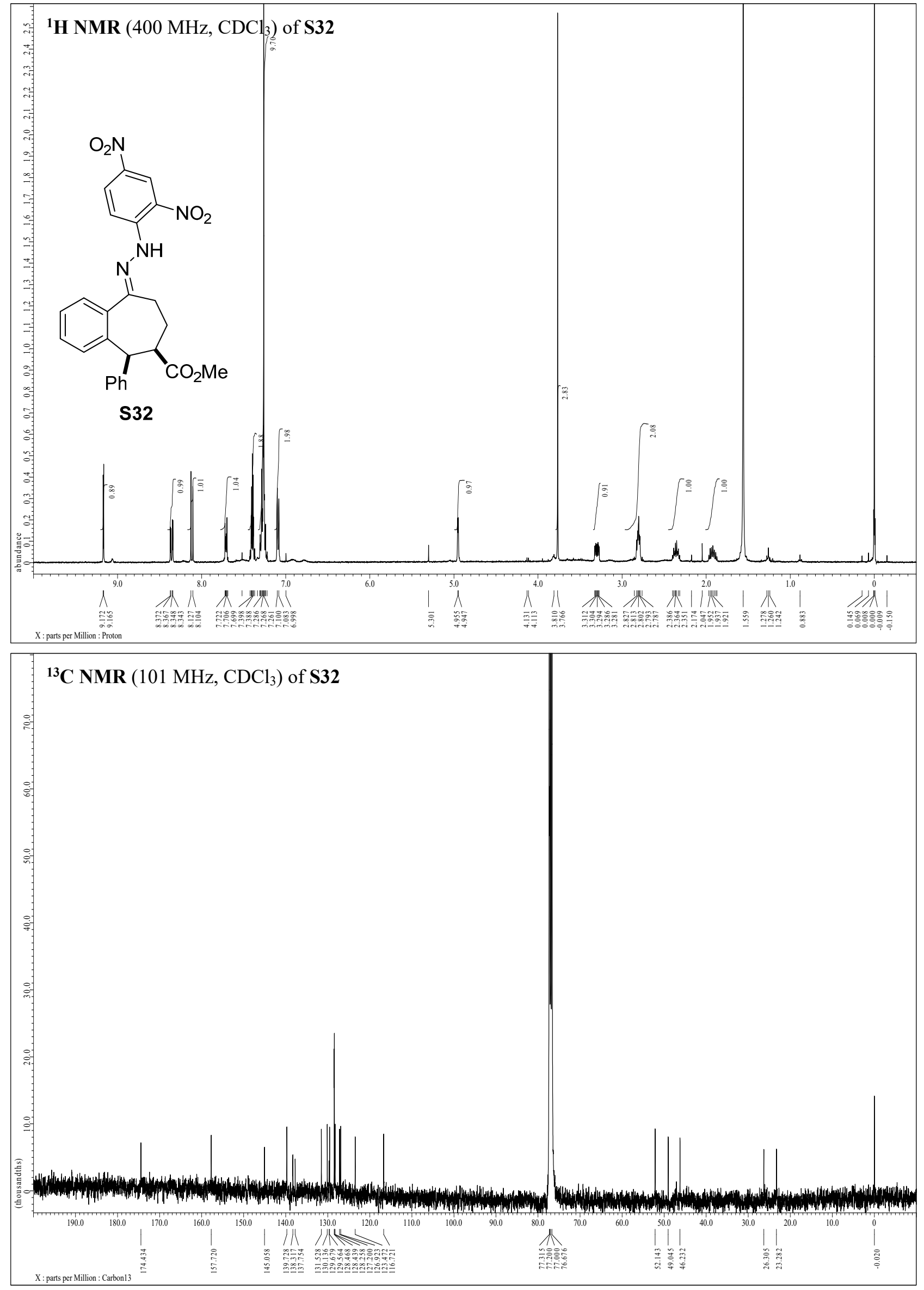